\title{
Natural Gas Imports and Exports \\ Fourth Quarter Report 1999
}

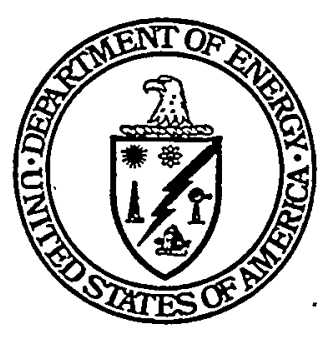

Prepared by:

U.S. Department of Energy

Office of Fossil Energy

Office of Natural Gas Petroleum

Import and Export Activities

Washington, DC 20585

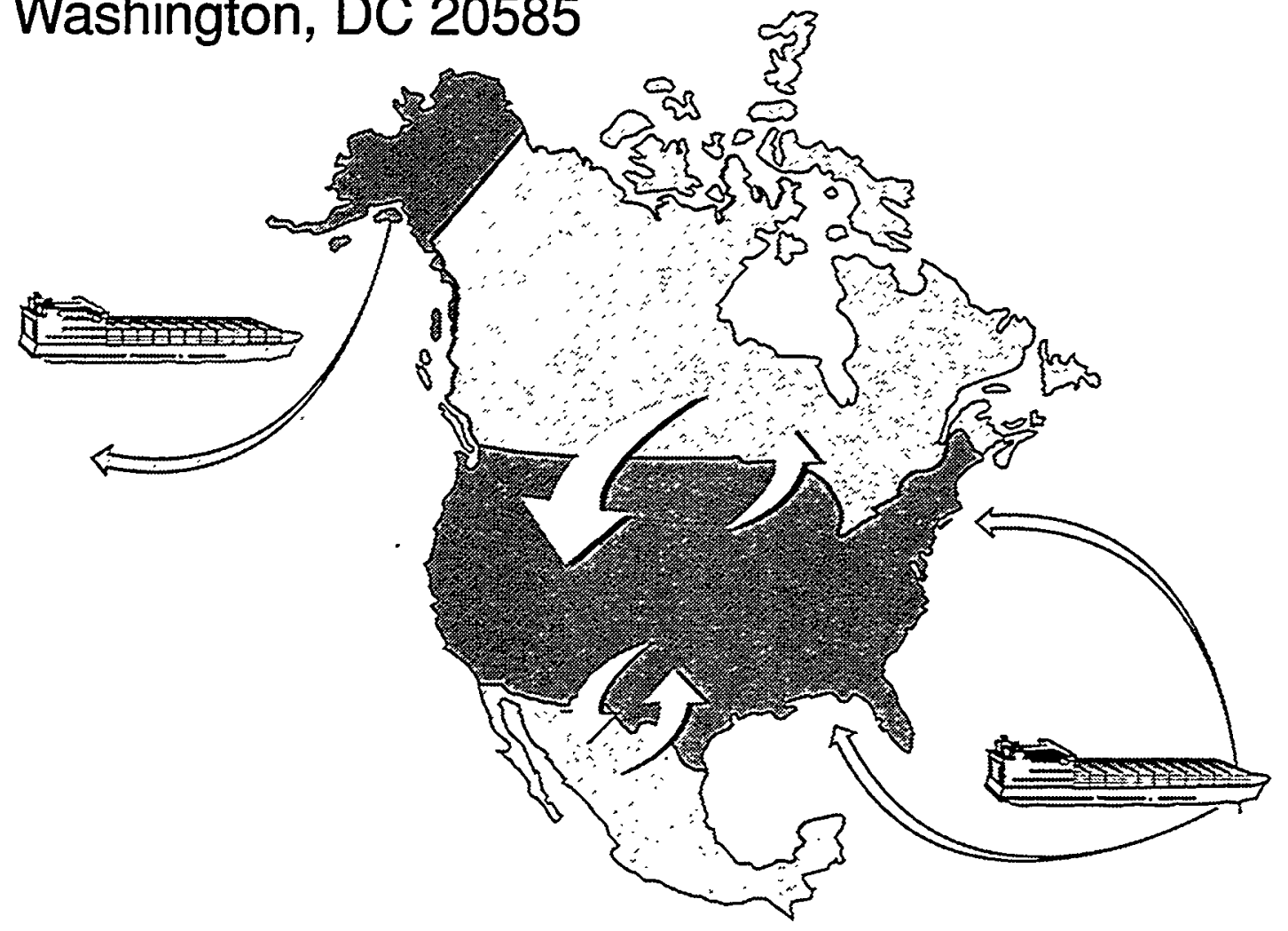

Printed with soy ink on recycled paper 


\section{DISCLAIMER}

This report was prepared as an account of work sponsored by an agency of the United States Government. Neither the United States Government nor any agency thereof, nor any of their employees, make any warranty, express or implied, or assumes any legal liability or responsibility for the accuracy, completeness, or usefulness of any information, apparatus, product, or process disclosed, or represents that its use would not infringe privately owned rights. Reference herein to any specific commercial product, process, or service by trade name, trademark, manufacturer, or otherwise does not necessarily constitute or imply its endorsement, recommendation, or favoring by the United States Government or any agency thereof. The views and opinions of authors expressed herein do not necessarily state or reflect those of the United States Government or any agency thereof. 


\section{DISCLAIMER}

Portions of this document may be illegible in electronic image products. Images are produced from the best available original document. 
The Office of Natural Gas \& Petroleum Import \& Export Activities prepares quarterly reports showing natural gas import and export activity. Companies are required, as a condition of their authorizations, to file quarterly reports. This report is for the fourth quarter of 1999 (October through December).

Attachment A shows the percentage of takes to maximum firm contract levels and the weighted average per unit price for each of the long-term importers during the five most recent reporting quarters.

Attachment $B$ shows volumes and prices of gas purchased by long-term importers and exporters during the past 12 months.

Attachment $C$ shows volume and price data for gas imported on a short-term or spot market basis.

Attachment $D$ shows the gas exported on a short-term or spot market basis to Canada and Mexico.

Fourth Quarter Highlights: As illustrated below, there were notable changes in activity compared to the fourth quarter of 1998.

\begin{tabular}{|lrl|}
\hline Canadian Imports & $860.8 \mathrm{Bcf}$ & up 12\% \\
LNG Imports & $42.6 \mathrm{Bcf}$ & up $51 \%$ \\
Mexican Imports & $13.9 \mathrm{Bcf}$ & up $504 \%$ \\
Total Imports & $917.3 \mathrm{Bcf}$ & up $14.6 \%$ \\
Mexican Exports & $13.1 \mathrm{Bcf}$ & down $16 \%$ \\
Canadian Exports & $17.6 \mathrm{Bcf}$ & up $46.6 \%$ \\
Japanese Exports & $14.9 \mathrm{Bcf}$ & down $.1 \%$ \\
\hline
\end{tabular}

See details below for more information.

Two new long-term import authorizations were activated, totaling $50 \mathrm{MMcf}$ per day or $18.25 \mathrm{Bcf}$ annually. Most of these volumes (43.9 MMcf per day or nearly 88 percent) will supply a $150 \mathrm{MW}$ cogeneration facility near Jay, Maine. The steam and electricity produced by this cogen facility will be sold to International Paper Company's Androscoggin Mill. Any excess electricity or imported gas will be sold to the New England Power Pool or other third parties. The other 12 percent of the new imports (or 6.1 MMcf per day) will supply industrial, commercial and residential customers in Duluth, Minnesota.

During the fourth quarter, 4 importers of LNG (Distrigas Corporation; Duke Energy LNG; CMS Marketing, Services and Trading Company; and Enron International Gas Sales Company) brought in 10 spot cargoes from 5 different countries (Algeria, Australia, Qatar, Trinidad and Tobago, and United Arab Emirates), totaling 20.8 Bcf.

Fourth Quarter Data: Comparing fourth quarter 1999 to fourth quarter 1998, total imports under long-term contracts declined slightly. Of this total, long-term Canadian imports also declined slightly while long-term $L N G$ imports increased significantly. Specifically, natural gas imports under all long-term contracts totaled $357.5 \mathrm{Bcf}$, compared to $358 \mathrm{Bcf}$ in the fourth quarter of 1998 . Of this total, long-term Canadian imports were down 1 percent ( 335.7 v. 340.4 $\mathrm{Bcf})$. The average price of this gas was $\$ 2.56$ per MMBtu, which is 12 cents or 5 percent higher than the preceding quarter and 42 cents or 20 percent higher than the fourth quarter of 1998. Long-term LNG imports increased 24 percent ( 21.8 v. $17.6 \mathrm{Bcf})$, reflecting the new long-term supply arrangement with Trinidad that began in May 1999. Under long-term LNG import contracts, Distrigas imported $7.4 \mathrm{Bcf}$ from Algeria at an average price of $\$ 2.61$ per MMBtu and 11.9 Bcf from Trinidad at \$2.73. Duke imported 2.5 Bcf from Algeria at $\$ 2.00$.

During the fourth quarter, 94 companies used short-term authorizations to import $559.8 \mathrm{Bcf}$, which is 26.5 percent more than the fourth quarter of 1998 (442.4 Bcf) but 2 percent less than last quarter (570.8 Bcf). Of this total, 525.1 Bcf was imported from Canada at an average price of $\$ 2.47$ per MMBtu, compared to $429.2 \mathrm{Bcf}$ at $\$ 1.91$ in the fourth quarter of 1998 , and $532.6 \mathrm{Bcf}$ at $\$ 2.27$ last quarter. Imports from Mexico totaled 13.9 Bcf at $\$ 2.31$, compared to $2.3 \mathrm{Bcf}$ at $\$ 1.70$ in the fourth quarter of 1998 and $14.5 \mathrm{Bcf}$ at \$2.44 last quarter. . Short-term LNG imports totaled 20.8 Bcf for the quarter, compared to $10.6 \mathrm{Bcf}$ in the fourth quarter of 1998 and 23.7 Bcf last quarter. Under these short-term contracts, Distrigas imported 5.9 Bcf from Trinidad at \$2.42 per MMBtu; Duke imported 2.7 Bef from Algeria at \$2.00; CMS imported 4.7 Bcf from Australia at \$2.24; Enron imported 2.7 Bcf from UAE at \$2.69; and CMS imported 4.8 Bcf from Qatar at \$3.13.

Approximately 31 percent of the short-term Canadian imports occurred at Eastport, ID at an average price of $\$ 2.42$ per MMBtu; 24 percent at Port of Morgan, MT at $\$ 2.37 ; 16$ percent at Sumas, WA at $\$ 2.46 ; 14$ percent at Noyes, MN at \$2.54; 7 percent at Niagara Falls, NY at \$2.78; 4 percent at Waddington, NY at $\$ 2.59$; and 4 percent at other entry points at $\$ 2.62$.

In addition, 15 short-term export authorizations were used, exporting a total of 30.7 Bcf of gas. Eight companies exported 17.6 Bcf to Canada, at an average price of $\$ 2.63$ per MMBtu. Seven companies exported 13.1 Bcf to Mexico at $\$ 2.66$. Finally, $14.9 \mathrm{Bcf}$ of $\mathrm{LNG}$ was exported to Japan at $\$ 3.55$ per MMBtu (delivered).

1999 Highlights: Comparing the 1999 data to that of 1998 , total gas imports increased by 12.6 percent $(3,549.3 \mathrm{v}$. $3,152.8 \mathrm{Bcf}$ ) and total gas exports increased by 2 percent (167.3v. 164.4Bcf). Based on preliminary gas consumption figures, net imports as a percentage of total domestic gas demand grew to 15.8 percent this year compared to 14 percent last year. Canadian imports increased by $279 \mathrm{Bcf}$ or 9 percent $(3,331.4$ v. $3,052.4$ Bcf); Mexican imports increased 276 percent ( $54.5 \mathrm{v}$. $14.5 \mathrm{Bcf}$ ); and LNG imports increased 90 percent ( 163.4 v. $85.9 \mathrm{Bcf}$ ). Exports to Canada decreased 6 percent ( 42.4 v. $45.3 \mathrm{Bcf}$ ) and exports to Mexico increased 15 percent (61.3 v. 53.1 Bcf). LNG exports to Japan decreased nearly 4 percent (63.6 V. 66 Bcf).

This quarter's focus report is a review of gas import/export activity for 1999. Next quarter's focus report will go into further detail of 1999's gas trade. The quarterly report and future revisions to the report will reside on our Electronic Bulletin Board at (202) 586-7853 and on the Fossil Energy Web Site at http://www.fe.doe.gov (click on Regulatory Natural Gas). Any questions or comments about this report should be directed to Yvonne. Caudillo at (202) 586-4587 or by E-mail at yvonne.caudillo@hq.doe.gov. 
NATURAL GAS IMPORTS

Volumes and Weighted Average Prices

By Point of Entry and Type of Authorization

$(10 / 01 / 99$ - 12/31/99)

VOLUME

(MMCF)

WEIGHTED

AVERAGE

PRICE

(\$MMMTU)

\section{POINT OF ENTRY}

Detroit, Michigan

Eastport, Idaho

Everett, Massachusetts

Grand Island, New York

Highgate Springs, Vermont

International Falls, Minnesota

Lake Charles, Louisiana

Massena, New York

Niagara Falls, New York

Noyes, Minnesota

Pittsburg, New Hampshire

Port of Morgan, Montana

Portal, North Dakota

St. Clair, Michigan

Sumas, Washington

Waddington, New York

Warroad, Minneșota
Babb, Montana

Champlain, New York

\section{LONG-TERM AUTHORIZATIONS}

$\begin{array}{rl}0.16 & 3.18 \\ 4,265.26 & 3.70 \\ 2,125.22 & 2.51 \\ 55,621.80 & 2.25 \\ 19,330.01 & 2.68^{*} \\ 3,556.82 & 4.02 \\ 2,183.88 & 3.35 \\ 183.86 & 2.45 \\ 2,472.78 & 2.00^{* *} \\ 1,045.22 & 3.13 \\ 43,987.70 & 2.82 \\ 57,087.94 & 2.52 \\ 6,194.89 & 2.68 \\ 75,587.70 & 2.26 \\ 112.76 & 1.19 \\ 2,886.24 & 2.92 \\ 22,535.27 & 2.54 \\ 58,215.24 & 2.87 \\ 114.45 & 2.49 \\ & \\ 357,507.20 & 2.57\end{array}$

\section{SHORT-TERM AUTHORIZATIONS}

\author{
Alamo, Texas \\ Babb, Montana \\ Champlain, New York \\ Detroit, Michigan \\ Eastport, Idaho \\ Everett, Massachusetts \\ Grand Island, New York \\ Hidalgo, Texas \\ Highgate Springs, Vermont \\ Lake Charles, Louisiana \\ Massena, New York \\ Niagara Falls, New York \\ Noyes, Minnesota \\ Pittsburg, New Hampshire \\ Port of Del Bonita, Montana \\ Port of Morgan, Montana \\ Portal, North Dakota \\ St. Clair, Michigan \\ Sumas, Washington \\ Waddington, New York \\ Warroad, Minnesota \\ Whitlash, Montana
}

$\begin{array}{rl}11,669.50 & 2.32 \\ 488.82 & 2.33 \\ 6.83 & 2.58 \\ 206.67 & 2.26 \\ 161,473.91 & 2.42 \\ 5,873.53 & 2.42^{*} \\ 5,466.08 & 2.75 \\ 2,258.06 & 2.24 \\ 245.31 & 2.50 \\ 14,881.18 & 2.50^{* *} \\ 1,018.98 & 3.12 \\ 39,772.76 & 2.78 \\ 70,976.35 & 2.54 \\ 3,282.82 & 2.73 \\ 3,697.59 & 2.19 \\ 126,338.82 & 2.37 \\ 289.18 & 2.14 \\ 5,903.51 & 2.72 \\ 82,498.68 & 2.46 \\ 21,371.51 & 2.59 \\ 1,389.24 & 2.64 \\ 650.42 & 2.33\end{array}$

$559,759.74$

* Average landed price

* Average tailgate price

Note: Import figures in this table may vary slightly from sum of components in report due to independent rounding in calculations. 


\section{QUARTERLY FOCUS: 1999 YEAR IN REVIEW}

Table 1

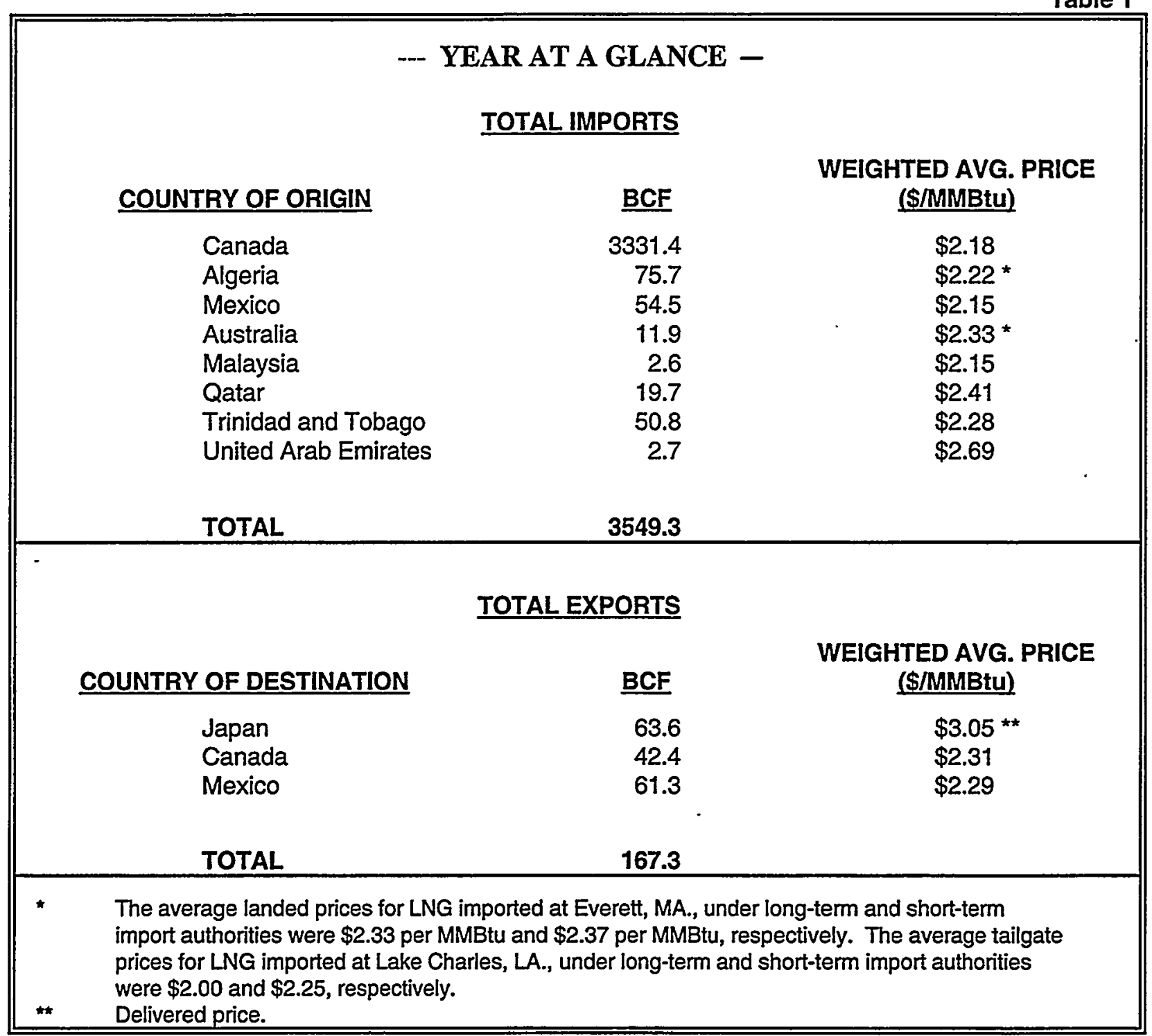

- Table 1 shows the volumes and prices of natural gas imports by country of origin, and natural gas exports by country of destination for 1999. The weighted average price for imports is the per unit price (MMBtu) at the point of entry into the United States. The price shown for exports is at the point of exit, with the exception of sales to Japan; the price of exports to Japan is shown as a delivered price.

- Natural gas imports, for the twelfth consecutive year, reached an historic high in 1999. The United States imported 3,549.3 billion cubic feet (Bcf) and exported 167.3 Bcf of natural gas, resulting in net imports of 3,382 Bcf for the year. This represents an increase of $393.5 \mathrm{Bcf}$, or 13 percent over the net import 1998 level $(2,988.5 \mathrm{Bcf})$.

- In 1999, natural gas exports increased by $3 \mathrm{Bcf}$, or 2 percent from the 1998 level (167.3 v. 164.4 Bcf). Exports to Mexico rose 15 percent, while exports to Canada and Japan saw moderate decline. 


\section{Natural Gas Import and Export Activity 1986 - 1999}
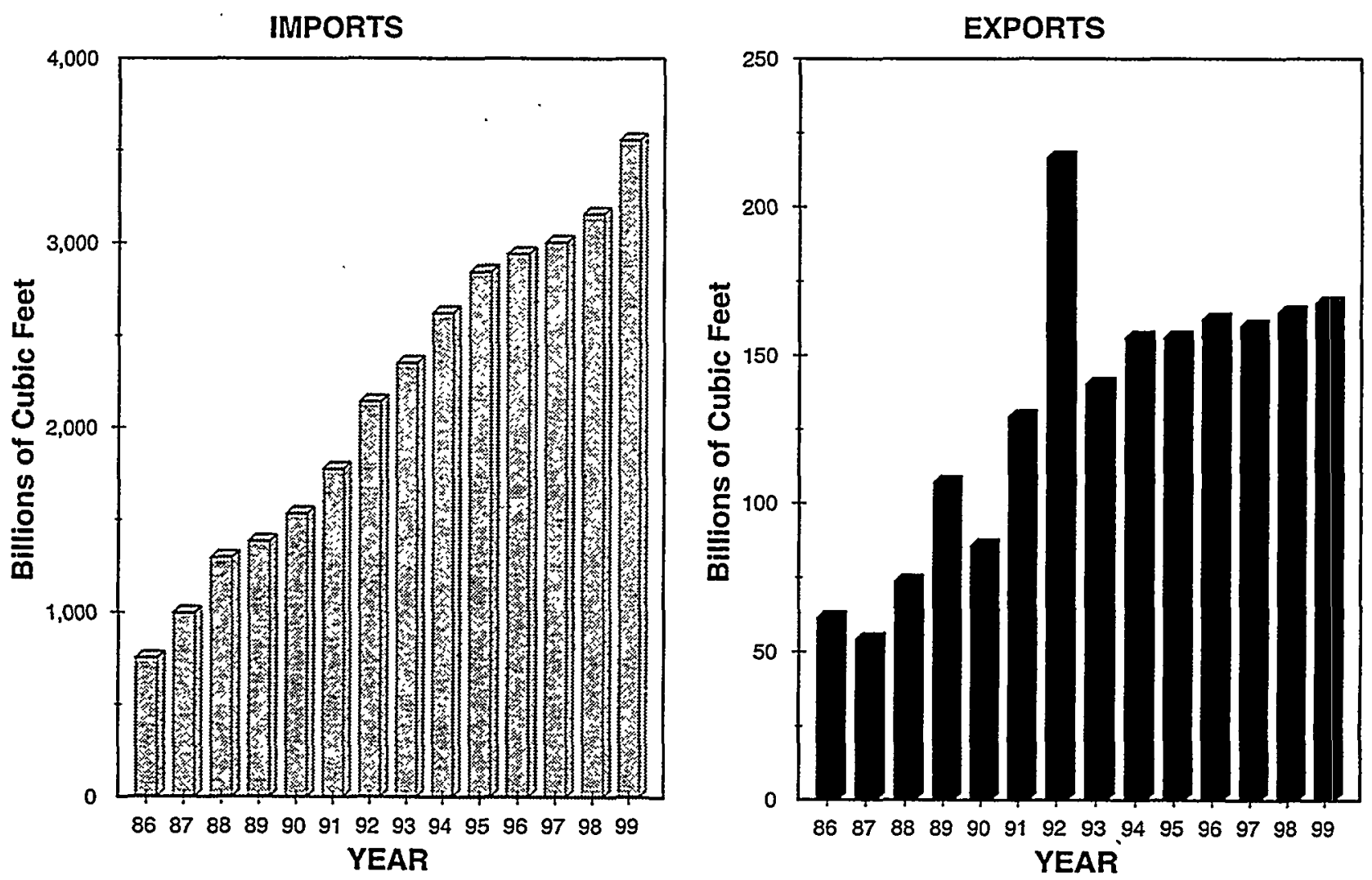

- Figure 1 shows natural gas import and export activity over the past 14 years (1986-1999).

- From 1986 to 1999, gross imports have grown by over 373 percent (750 Bcf v. 3,549 Bcf). Additionally, net imports (imports minus exports) as a percentage of total domestic gas demand have grown from 4.2 percent in 1986 to an estimated 15.8 percent in 1999. The surge in market share for gas imports in 1999 was due to the fact that domestic demand for natural gas grew by only one percent while net imports increased by 13 percent.

- Total gross imports into the U.S. increased by $396 \mathrm{Bcf}$, or 12.6 percent over last year's level (3,549 Bcf v. 3,153 Bcf in 1998). The 12.6 percent increase in imports is the first time in five years imports have increased by double digits and the largest percentage increase since 1992, when the Iroquois Gas Transmission System became operational. On a volumetric basis, the $396 \mathrm{Bcf}$ represents the largest ever year-to-year increase in imports. The large gain in import volumes came from a combination of events. During the year, there was a nine percent growth in Canadian supplies (largely due to the Northern Border Pipeline expansion), a 90 percent increase in LNG supplies (due to a new supply from Trinidad and a brisk spot market) and a 279 percent increase in Mexican volumes (54.5 v. 14.5 Bcf). 


\section{Estimated Sales of Imported Natural Gas By Census Division - 1998 and 1999 \\ (Billions of Cubic Feet)}

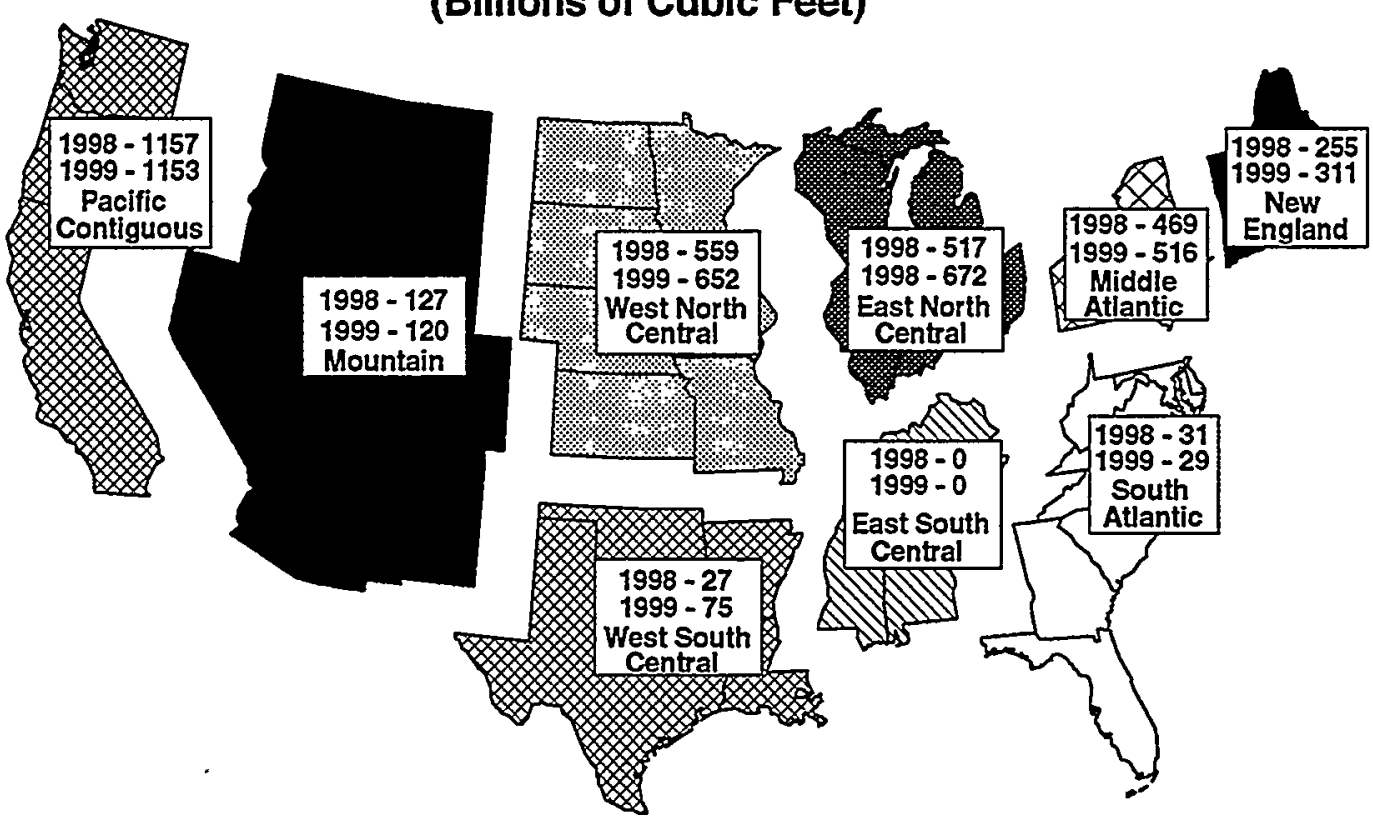

Note: Import sales do not equal Imports due to fuel use, linepack, storage, Imbalances and undetermined markets.

- Exports were at their highest level since 1992 and totaled 167.3 Bcf. During 1999, about 38 percent $(63.6 \mathrm{Bcf})$ of the gas exports were shipped to Japan, 37 percent $(61.3 \mathrm{Bcf})$ of the volumes were exported to Mexico, and 25 percent of the volumes ( $42.4 \mathrm{Bcf}$ ) were exported to Canada. As shown in Figure 1, the largest volume of gas exports occurred in 1992; this historic high level of exports was the result of record export sales to both Canada and Mexico.

- During 1999, natural gas imports into the United States continued their historic growth. As illustrated in Figure 2, five of the nine Census regions shown above experienced increased use of natural gas imports. The greatest volumetric increases occurred in the upper Midwest as Region 3 (East North Central) and Region 4 (West North Central) experienced increases of 155 Bcf and 93 Bcf, respectively. This growth was the direct result of the expansion of the Northern Border Pipeline. The expansion of this facility allowed for an additional 248 Bcf of Canadian natural gas to be marketed in these two Regions.

- The growth in natural gas imports depicted in Region 1 (New England) was almost totally attributable to increased sales of LNG, mostly from Algeria and Trinidad. Beginning in May 1999, Trinidad became a major suppler of LNG to the New England region. In 1999, Canadian natural gas supplies marketed in this region were only slightly higher than the 1998 level. The geographic area which had the largest year-to-year growth on a percentage basis was Region 7 (West South Central). The growth reflects the increased volumes of Mexican natural gas exported to the U.S. during the year. 


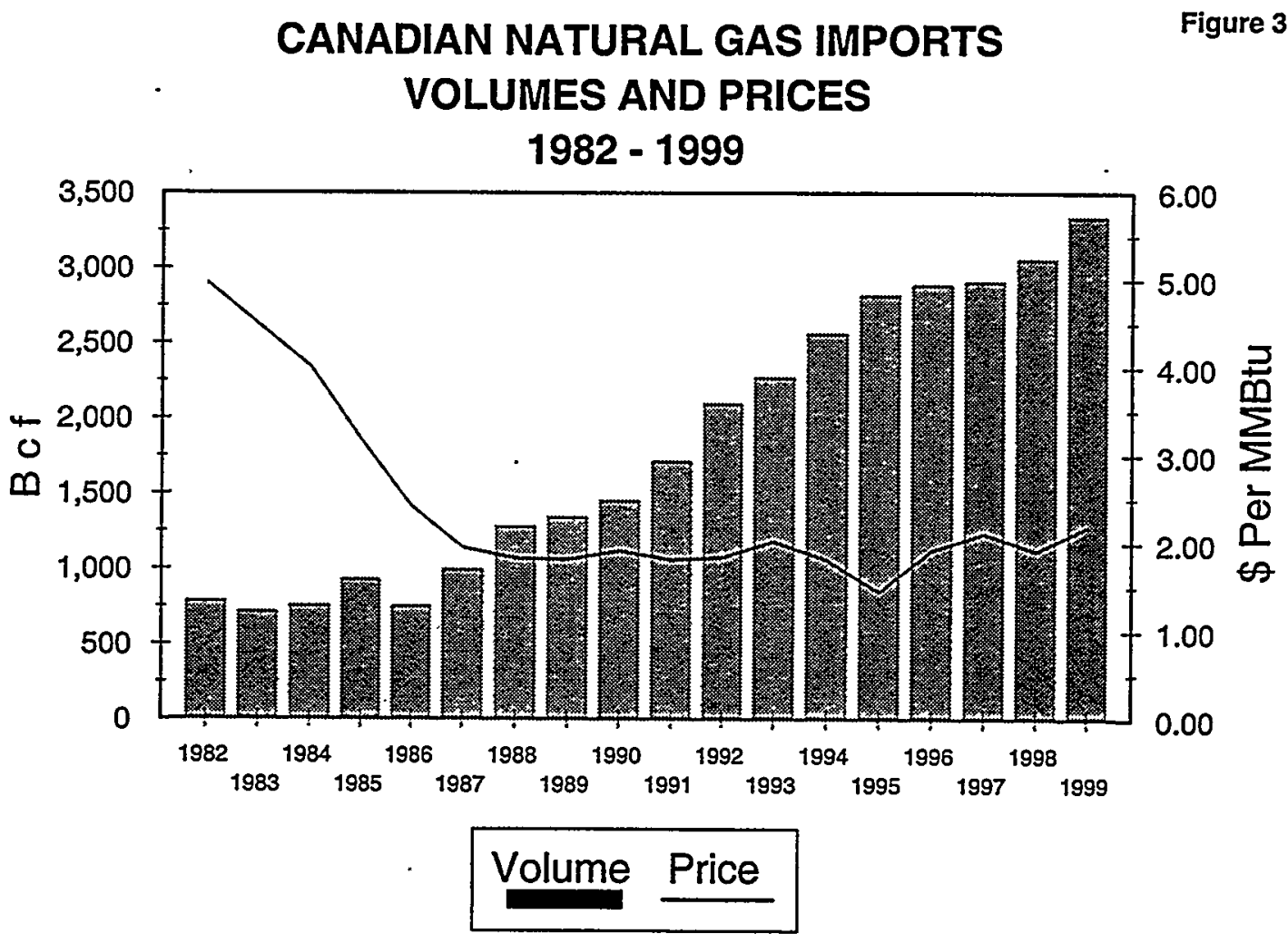

- Figure 3 shows the volume and price trend for Canadian natural gas imports during the past 18 years.

- Canadian natural gas imports in 1999 grew by 279 Bcf and established a new record at 3,331.4 Bcf. The rate of growth from the 1998 level was 9 percent, representing the biggest gain in volumes since 1995 when Canadian imports grew by 10 percent. The average international border price for Canadian gas supplies in 1999 was $\$ 2.18$ per MMBtu. This price was 14 percent higher than last year's average price of $\$ 1.91$ per MMBtu.

- The increased price for gas supplies in 1999 has resulted in a significant increase in revenues for Canadian gas producers. In 1999, it is estimated that Canadian gas revenues reached $\$ 7.3$ billion; this compares with estimated 1998 revenues of $\$ 5.8$ billion.

- The average price of gas imported from Canada in 1999 was $\$ 2.28$ per MMBtu under longterm contracts (contracts longer than 2 years) and $\$ 2.12$ per MMBtu under short-term contracts (contracts of 2 years or less).

- During 1999, Canada's share of the natural gas import market in the United States was 93.9 percent. LNG imports from Algeria, Australia, Malaysia, Qatar, Trinidad and Tobago, and the United Arab Emirates comprised about 4.6 percent of the import market, and Mexico's share equaled about 1.5 percent. 


\section{Canadian Natural Gas Imports By Point of Entry}

Figure 4
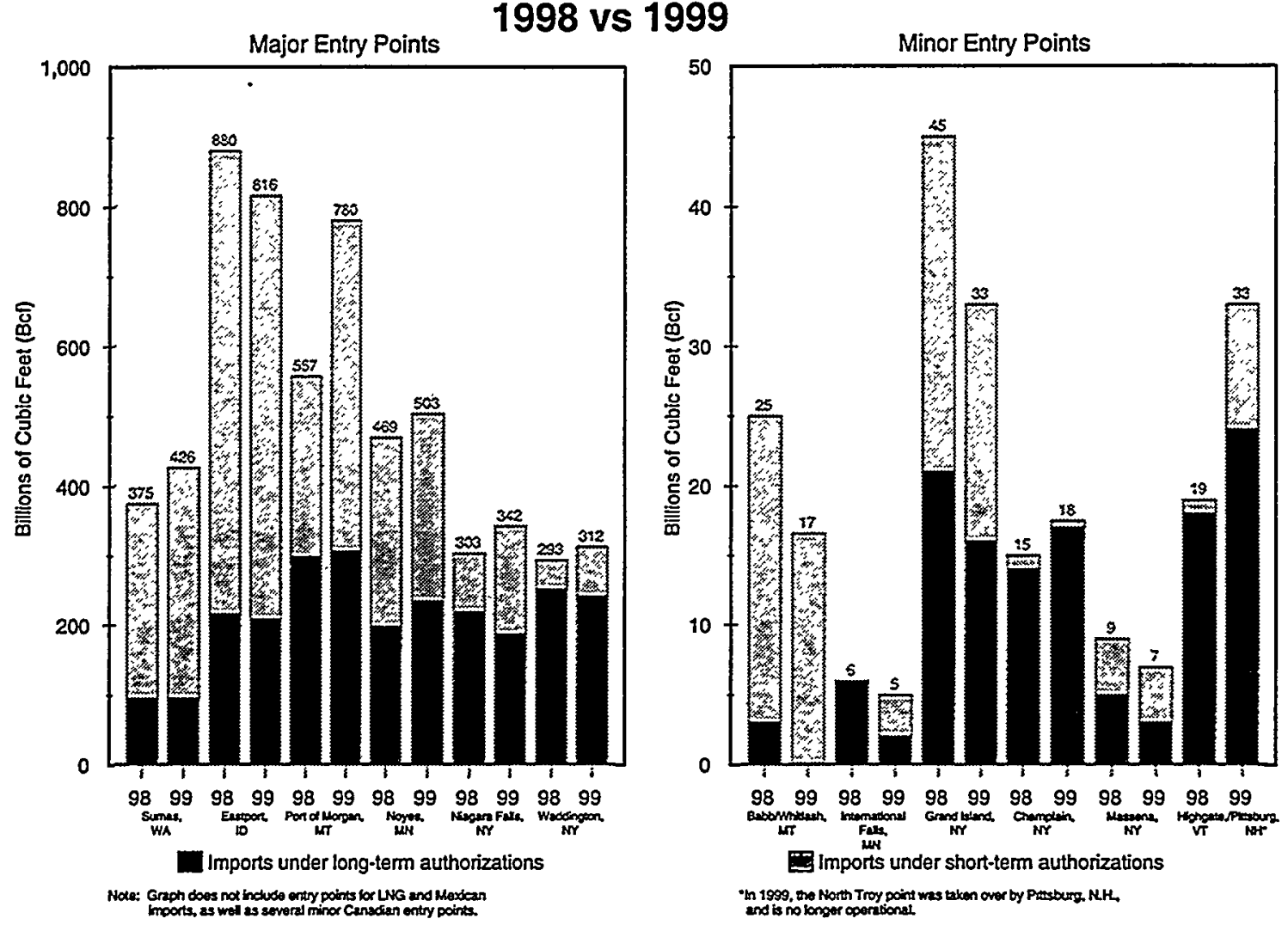

Figure 4 compares natural gas imports from Canada by point of entry for 1998 and 1999 and distinguishes between imports made under short-term and long-term import authorizations.

Figure 4 shows increased levels of imports at most major import points. During 1999, the international border point of Port of Morgan, Montana, showed the largest increase in volumes (up 36\%). This surge in activity was the direct result of the new Northern Border Pipeline Expansion, which commenced service on December 22, 1998. The newly operational pipeline increased Northern Borders's pipeline capacity by $700 \mathrm{MMcf}$ per day, allowing an additional $612 \mathrm{MMcf}$ per day to flow at this point in 1999. In addition, import levels at other major entry points rose significantly this year: Sumas, Washington (up 14\%); Niagara Falls, New York, (up 13\%); Noyes, Minnesota, (up 9\%); and Waddington, New York, (up 6\%).

- Natural gas imports at most of the minor points of entry on the U.S.- Canada international border showed declines in 1999 from the preceding year. Fewer spot sales to the Rocky Mountain states resulted in reduced imports at the Montana/Canada border. Reduced imports at two of the entry points in the State of New York reflect the termination of numerous longterm supply contracts with cogeneration facilities, as well as local utilities, located in the State of New York. The termination of these contracts was the direct result of the electric restructuring efforts by the State over the past few years. This year's data for the two points of entry into the States of New Hampshire and Vermont were previously both located in Vermont (Highgate Springs and North Troy). The North Troy import point no longer exists and has essentially been replaced by the Pittsburg, New Hampshire entry point. 


\section{Natural Gas Exports To Mexico By Point of Exit}

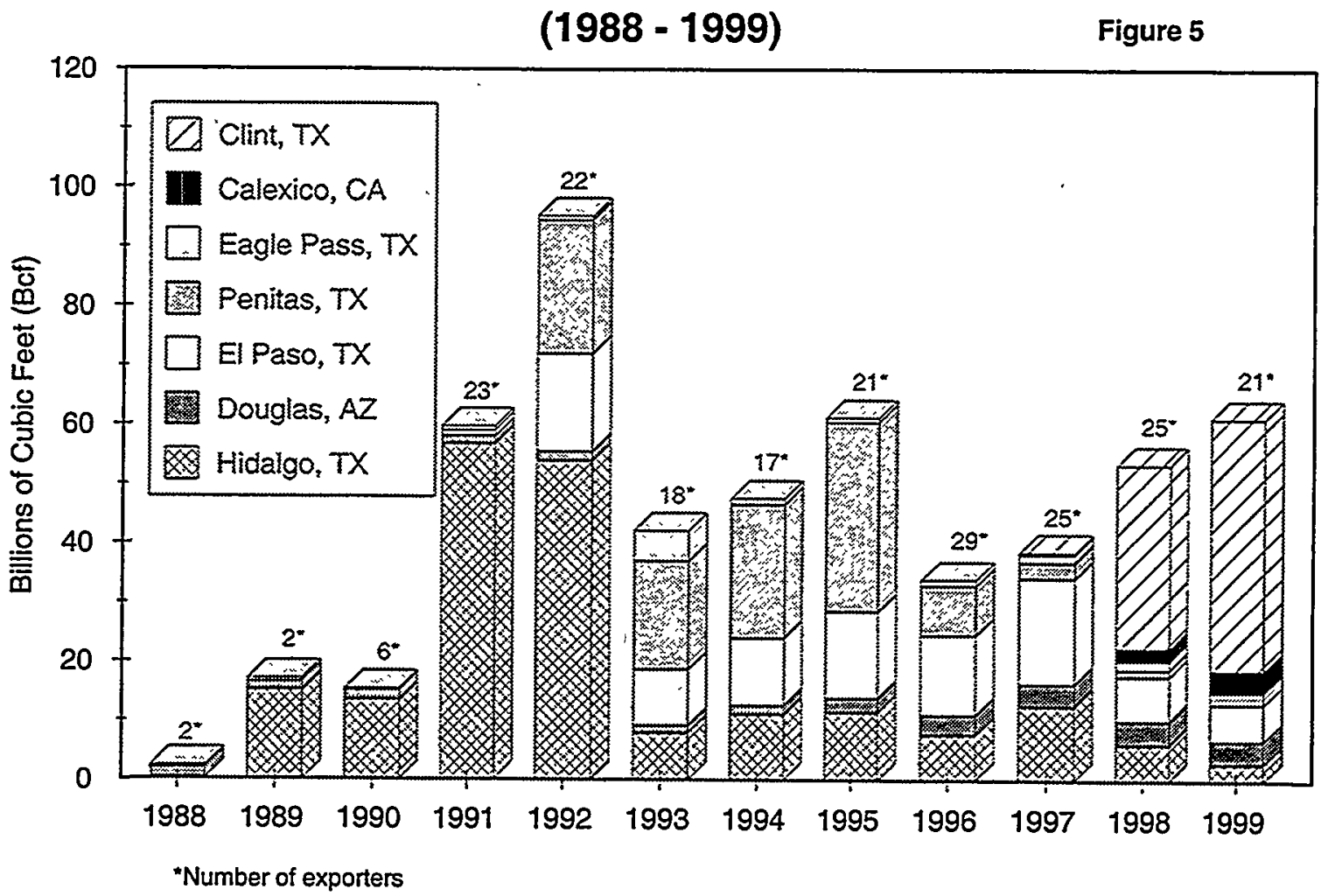

During 1999, 21 companies exported 61.3 Bcf of natural gas to Mexico, the highest level of annual exports since 1992. As shown in Figure 5, more than two-thirds of the volumes this year were exported at Clint, Texas, on the Samalayuca pipeline, which commenced operation on December 20, 1997. During the second quarter, the volumes exported at Clint totaled a sizeable $12.1 \mathrm{Bcf}$, and contributed toward making this location the leading point of export for the second year in a row. In addition, 277.5 MMcf of LNG was exported this year, via truck, to Nogales, Sonora, and Baja California, Mexico.

- The weighted average price of 1999 exports to Mexico was $\$ 2.29$ per MMBtu and was 13 percent higher than last year's average price of $\$ 2.02$ per MMBtu. In 1998, the weighted average price fell to its lowest level since 1995 , when the price was $\$ 1.48$ per MMBtu.

- During the second quarter of 2000 , it is likely that a new international border crossing facility will become operational and begin exporting natural gas to Mexico at Otay Mesa, California. The 30-inch diameter pipeline, which is being built by the San Diego Gas and Electric Company (an affiliate of Sempra Energy), will have the capacity to export up to 300 million cubic feet of natural gas per day to various customers in Northern Baja California. On March 16, 2000, Gasoducto Rosarito, S. de. R.L. de C. V., an indirect wholly-owned subsidiary of 
Sempra Energy, filed an application with our office seeking short-term, blanket authority to export up to $155 \mathrm{Bcf}$ over a two year period on this new pipeline facility to serve various markets, including power generators, commercial and industrial end-users, and local distribution companies.

- During 1999 imports from Mexico increased 276 percent over the 1998 level (54.5 Bcf v. 14.5 $\mathrm{Bcf})$. The average international border price for Mexican gas supplies was $\$ 2.15$ per MMBtu. This price was 12 percent higher than last year's average price of $\$ 2.01$ per MMBtu. It is interesting to note that during the second quarter, the volumes imported from Mexico approached the amount exported to Mexico for that period (16.0 Bcf v. $16.5 \mathrm{Bcf})$.

- During the year, most of the import volumes were brought into the United States on the Texas Eastern pipeline at Hidalgo, Texas; however, in the fourth quarter of 1999, the principal pipeline facility bringing in gas imports is the newly operational Tennessee Pipeline, located near Alamo, Texas. This bi-directional pipeline commenced operation in late September.

- Figure 6 on the following page is a map showing the identity and location of the eight existing natural gas pipelines enabling cross-border trade between the United States and Mexico. The Table included with Figure 6 estimates the daily design capacities in millions of cubic feet (MMcf) for all of the pipelines and provides their actual average daily throughput from 1992 through 1999. Our Office currently estimates that the average aggregate throughput capacity of these eight pipelines totals 1370 MMcf per day, or 500 billion cubic feet (Bcf) per year. 


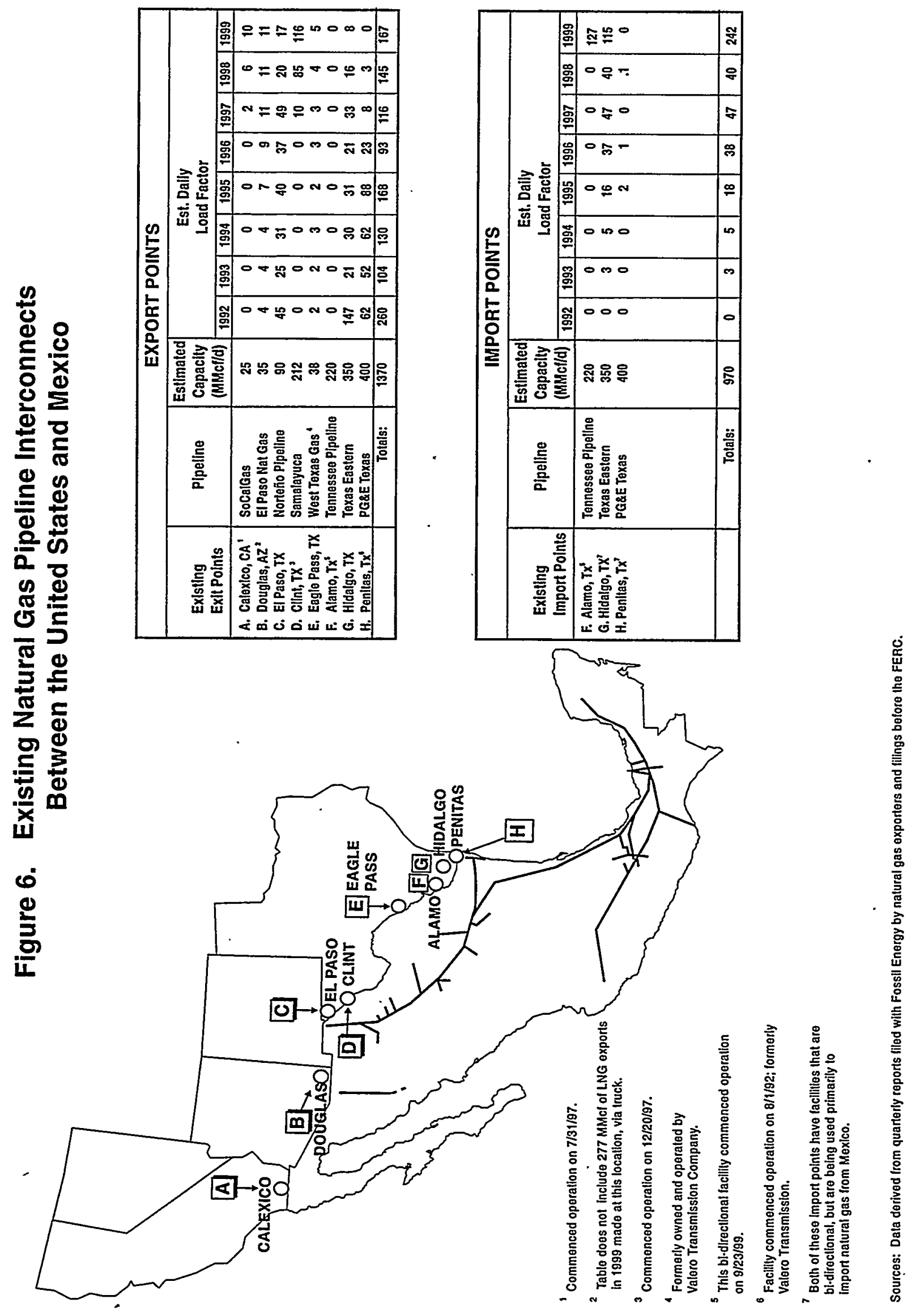

North American Gas Trade * North American Gas Trade * North American Gas Trade * North American Gas Trade * North American Gas Trade viii 


\section{LNG TRADE}

\section{LNG TRADE}

Figure 7

\section{8 vs 1999}
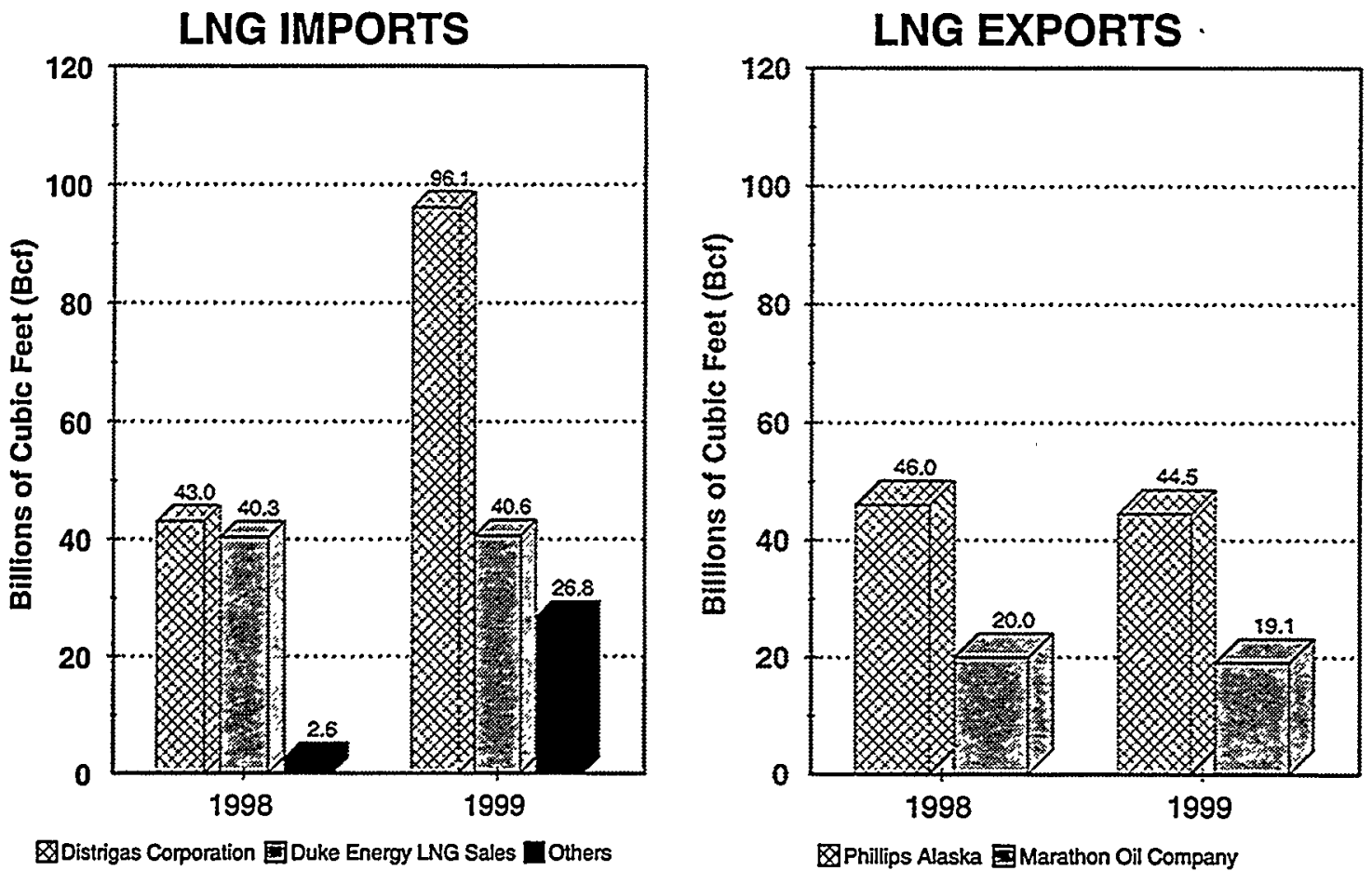

- $\quad$ Figure 7 compares imports and exports of liquefied natural gas (LNG) for 1998 and 1999.

- During 1999, five companies, led by Distrigas Corporation (Distrigas) and Duke Energy LNG Gas Sales, Inc. (Duke Energy), imported a record 163.4 Bcf of LNG into the United States. As shown in Figure 7, total LNG imports in 1999 increased by 90.2 percent from the 1998 level (163.4 v. $85.9 \mathrm{Bcf}$ ). Imports by Distrigas into its Everett, Massachusetts, terminal rose an impressive 123.2 percent (96.1 v. 43.0), and imports by Duke Energy into CMS Energy's Lake Charles, Louisiana, terminal increased slightly (40.6 v. 40.3). In addition, three companies made spot purchases of $26.8 \mathrm{Bcf}$ at the Lake Charles terminal this year: Enron International Gas, Coral Energy and CMS Marketing. The growth of LNG spot sales by new importers increased by $24.2 \mathrm{Bcf}$ from last year, when Enron imported 1 spot cargo of $2.6 \mathrm{Bcf}$.

- Table 2 on the following page shows a detailed listing of 1999 imports of LNG. During 1999, a total of 72 cargoes of LNG were imported into the United States. Distrigas imported a total of 45 cargoes; it purchased 17 cargoes from Algeria under two long-term import authorizations and 18 cargoes from another long-term import authorization involving the newly operational Trinidad and Tobago LNG facility. In addition, Distrigas imported 9 spot cargoes from Trinidad and 1 spot cargo from Australia. The total number of cargoes imported this year by Distrigas increased by $165 \%$ over last year's shipments ( 45 v. 17 cargoes). 
North American Gas Trade * North American Gas Trade * North American Gas Trade * North American Gas Trade * North American Gas Trade

1999 Imports of Liquefied Natural Gas

Table 2

\begin{tabular}{|c|c|c|c|c|c|}
\hline $\begin{array}{l}\text { Name of } \\
\text { Importer }\end{array}$ & $\begin{array}{c}\text { Country of } \\
\text { Origin }\end{array}$ & $\begin{array}{c}\text { Number of } \\
\text { Cargoes }\end{array}$ & $\begin{array}{l}\text { Receiving } \\
\text { Terminal }\end{array}$ & $\begin{array}{l}\text { Volume } \\
\text { (Mcf) }\end{array}$ & $\begin{array}{l}\text { Avg. Price } \\
\text { (\$/MMBtu) }\end{array}$ \\
\hline CMS Marketing & Australia & 4 & Lake Charles, LA & $9,346,442$ & $\$ 2.13 *$ \\
\hline CMS Marketing & Qatar & 5 & Lake Charles, LA & $12,141,012$ & $\$ 2.69 *$ \\
\hline Coral Energy & Malaysia & 1 & Lake Charles, LA & $2,575,895$ & $\$ 2.15$ \\
\hline Distrigas Corp. & Algeria & 17 & Everett, MA & $42,734,526$ & $\$ 2.36$ \\
\hline Distrigas Corp. & Australia & 1 & Everett, MA & $2,556,896$ & $\$ 3.06$ \\
\hline Distrigas Corp. & Trinidad & 27 & Everett, MA & $50,777,738$ & $\$ 2.28$ \\
\hline Duke Energy & Algeria & 13 & Lake Charles, LA & $33,028,938$ & $\$ 2.04 *$ \\
\hline Duke Energy & Qatar & 3 & Lake Charles, LA & $7,556,475$ & $\$ 1.96 *$ \\
\hline Enron Int'l. Gas & U.A.E. & 1 & Lake Charles, LA & $2,713,384$ & $\$ 2.69$ \\
\hline & & 72 & & $163,431,306$ & $\$ 2.28$ \\
\hline
\end{tabular}

* Denotes tailgate price. All imports coming into Everett, MA., are shown at the "Ianded cost" while most imports coming into Lake Charles, LA, (except Coral and Enron) are shown at the "tailgate selling price." Imports by Coral and Enron are at "landed cost."

Duke Energy imported a total of 16 cargoes in 1999, the same as in 1998. This year Duke purchased 13 cargoes from Algeria (10 under its long-term authorization and 3 under its shortterm authorization), and 3 cargoes from Qatar under its short-term import authority. In addition, CMS Marketing purchased 9 spot market cargoes (5 from Qatar, and 4 from Australia), and Enron International Gas purchased 1 spot cargo from the United Arab Emirates. This year, LNG was imported for the very first time from Malaysia. In August, Coral Energy, importing gas for the first time, purchased 1 spot cargo at the Lake Charles, Louisiana, terminal.

The average landed price of Algerian LNG imported in 1999 by Distrigas under its long-term authorization was $\$ 2.36$ per MMBtu, a nine percent decrease from the 1998 price of $\$ 2.59$ per $M M B t u$, and the average landed price of LNG imported from Trinidad under new long-term arrangements was $\$ 2.30$. With respect to Duke Energy's purchases of Algerian LNG under a long-term contract, the average tailgate price in 1999 was $\$ 2.00$ per MMBtu, up four cents from last year's price of $\$ 1.96$ per MMBtu. Under short-term authorizations, the average landed prices paid by Distrigas, Coral Energy and Enron were $\$ 2.37, \$ 2.15$, and $\$ 2.69$ per MMBtu, respectively, and the average tailgate prices paid by Duke and CMS Marketing were $\$ 2.06$ and $\$ 2.45$. 
Table 3 below shows the growth and diversity of countries supplying LNG to the U.S. over the last 5 years and signifies the expansion of spot sales in this trade.

Table 3

\begin{tabular}{|c|c|c|c|c|c|}
\hline \multicolumn{6}{|c|}{$\begin{array}{c}\text { SPOT / SHORT-TERM SELLERS OF LNG } \\
\text { TO THE UNITED STATES } \\
\text { Billions of Cubic Feet (Bcf) }\end{array}$} \\
\hline COUNTRY & 1995 & 1996 & 1997 & 1998 & 1999 \\
\hline Australia & \multirow{6}{*}{5.1} & \multirow{6}{*}{4.9} & 9.7 & 11.6 & 11.9 \\
\hline United Arab Emirates & & & \multirow[t]{5}{*}{2.4} & 5.3 & 2.7 \\
\hline Algeria & & & & 2.7 & 10.1 \\
\hline Qatar & & & & & 19.7 \\
\hline Trinidad and Tobago & & & & & 13.2 \\
\hline Malaysia & & & & & 2.6 \\
\hline TOTAL SPOT MARKET CARGOES & 2 & 3 & 5 & 8 & 27 \\
\hline TOTAL LNG SPOT SALES & 5.1 & 4.9 & 12.1 & 19.6 & 60.2 \\
\hline$\%$ of total LNG Imports & 28.5 & 12.2 & 15.6 & 22.8 & 36.8 \\
\hline
\end{tabular}

- The trend in LNG supply is expected to continue as new import facilities are planning to be reopened in Maryland and Georgia. In January 2000, Columbia LNG Corporation took over sole ownership of the Cove Point LNG terminal, located in Lusby, Maryland. Cove Point plans to reopen its LNG marine terminal facilities by late 2001, pending approval by the FederalEnergy Regulatory Commission (FERC). In another important development, the Department of Energy, on January 21, 2000, authorized El Paso Merchant Energy-Gas, L.P. to import up to 82 Bcf per year of LNG from Trinidad and Tobago for over 22 years to its currently idle Elba Island, Georgia, terminal. The FERC is expected soon to issue a Certificate of Public Convenience and Necessity authorizing the recommissioning of the facility after 20 years of inactivity. In the past few months, the FERC has given a preliminary determination on nonenvironmental issues and most recently also decided that recommissioning the Elba Island facility would have no significant impacts on the environment. 
- The future demand for imported LNG is uncertain; however, recent announcements by the industry would seem to indicate substantial growth in the foreseeable future. One area of growth will likely be for power generation. For example, in January, Sithe Energies signed an agreement with Cabot LNG to purchase approximately $70 \mathrm{Bcf}$ per year of regasified LNG. The supply, equal to about $25 \mathrm{LNG}$ cargoes per year, will be used to fuel Sithe's 1,600 MW Mystic Station powerplant currently under construction at Everett, Massachusetts. The facility is expected to begin operation in 2002. Cabot's recent agreement with Sithe joins two other arrangements made over the past six months. One involves a multi-year agreement between Cabot and the Berkshire cogeneration facility, owned by El Paso Energy, which specifies deliveries of $25 \mathrm{MMcf}$ per day of $\mathrm{LNG}$. Another recent arrangement requires delivery of regasified LNG to a nearby $350-\mathrm{MW}$ powerplant. The plant, owned by Cabot's affiliate, Cabot Power, currently is being constructed near the LNG terminal. Together, Cabot's three new supply agreements will require an additional $100 \mathrm{Bcf}$ per year of LNG supplies to meet this increased demand.

- Another LNG project, EcoElectrica, L.P., a limited partnership between Enron Development Corporation and Edison Mission Energy, is expected to begin importing LNG from Trinidad and Tobago by mid-year. The gas will be used to fuel a 461-MW combined-cycle cogeneration plant located on the south coast of Puerto Rico near the city of Ponce. EcoElectrica has a longterm purchase contract with Cabot LNG for acquiring 10 cargoes per year (approximately 29 Bcf) over a 20-year period.

- On March 13, 2000, the government of Trinidad and Tobago announced the signing of an agreement with Atlantic LNG Company of Trinidad and Tobago supporting a billion-dollar expansion of the company's existing LNG production and export facility located in Point Fortin. The facility expansion (adding two more trains) will triple the production and export of LNG by the year 2003. Much of this future LNG supply will likely be marketed in the United States.

Figure 7 shows the volume of LNG exported by Phillips Alaska Natural Gas Corporation (Phillips) and Marathon Oil Company (Marathon) from Kenai, Alaska, to Japan during 1998 and 1999.

LNG exports to Japan decreased 3.6 percent this year from the 1998 level (63.6 v. $66.0 \mathrm{Bcf}$ ). The weighted average delivered price for these volumes in 1999 was $\$ 3.05$ per MMBtu, which represents a 6 percent increase over the 1998 price of $\$ 2.87$ per MMBtu. Last year's price was the lowest since 1979, when the average annual sales price for LNG delivered to Japan was $\$ 2.32$ per thousand cubic feet (EIA/DOE-0130, Natural Gas Monthly, Table SR9, page xxxii). In addition, LNG volumes totaling 277.5 MMcf were exported to Nogales, Sonora, Mexico, via truck, this year. 
Note: Data used in this report are from company filings made with the Office of Fossil Energy (FE). All 1998/99 year-to-year comparisons utilize FE data. One should be mindful of the fact that FE data is collected on an equity (sales) basis, rather than on a custody (physical movements) basis, as employed by the Energy Information Administration (EIA) in its reports. As a consequence, the data may have some minor variances. 
Page - 1

\section{LONG-TERM IMPORTS}

\section{ACTUAL IMPORTS AS A PERCENT OF AUTHORIZED VOLUMES}

IMPORTERIEXPORTER

\section{ANDROSCOGGIN ENERGY LLCNarious Suppliers} AVISTA CORPORATION/AEC OII \& Gas Company AVISTA CORPORATION/Engage Energy Canada AVISTA CORPORATION/Enron Capital \& Trade AVISTA CORPORATION/PanCanadian Petroleum Limited BAY STATE GAS COMPANY/Renaissance Energy Ltd. BEAR PAW ENERGY, L.L.C./Amoco Canada Resources Ltd. BOSTON GAS COMPANYIAlberta NE Gas (TCGS Ltd. 1) BOUNDARY GAS, INC./TransCanada Pipelines Limited BROOKLYN NAVY YARD COGEN. PARTNERS, L.P./Crestar Energy BROOKLYN NAVY YARD COGEN. PARTNERS, L.P.IPanCanadian Petroleum Ltd. BROOKLYN UNION GAS COMPANYIAlberta NE Gas (AEC Oil \& Gas Company) BROOKLYN UNION GAS COMPANYIAlberta NE Gas (TCGS Lid. 1) BROOKLYN UNION GAS COMPANYIAlberta NE Gas (TCGS Lid. 2) BROOKLYN UNION GAS COMPANYIAlberta Northeast Gas (ProGas) BROOKLYN UNION GAS COMPANYIAlberta Northeast Gas (Producers Marketing) BROOKLYN UNION OF LONG ISLAND/Alberta NE Gas (AEC Oil \& Gas) BROOKLYN UNION OF LONG ISLAND/AIberta NE Gas (TCGS Lid. 1) BROOKLYN UNION OF LONG ISLAND/Alberta NE Gas (TCGS LId. 2) BROOKL.YN UNION OF LONG ISLAND/Alberta Northeast Gas (ProGas) BROOKLYN UNION OF LONG ISLAND/Alberta Northeast Gas (Producers Marketing) CASCADE NATURAL GAS COPORATION/IGI Resources, Inc.

CASCADE NATURAL GAS CORPORATION/Duke Energy Trad. \& Mklg. Svcs., LLC CASCADE NATURAL GAS CORPORATION/Engage Energy Canada CASCADE NATURAL GAS CORPORATION/Engage Energy Canada CENTRAL HUDSON COMPANYIAlberta NE Gas (AEC Oil \& Gas Company) CENTRAL HUDSON COMPANY/Alberta NE Gas (TCGS LId. 1) CENTRAL HUDSON COMPANY/Alberta NE Gas (TCGS Lid. 2) CENTRAL HUDSON COMPANY/Alberta Northeast Gas (ProGas) CENTRAL HUDSON COMPANY/Alberta Northeast Gas (Producers Marketing) CHEVRON NATURAL GAS SERVICES, INC./Chevron Canada Resources CHEVRON U.S.A. INC./Chevron Canada Resources CHEVRON U.S.A. INC./Chevron Canada Resources CITY OF BURBANK, CALIFORNIAJEngage Energy Canada, L.P. CITY OF DULUTH, MINNESOTA/ProGas Limited

\begin{tabular}{|c|c|c|c|c|}
\hline $\begin{array}{c}1998 \\
\text { qtr } \\
\# 4\end{array}$ & $\begin{array}{c}1999 \\
\text { gtr } \\
\# 1\end{array}$ & $\begin{array}{c}1999 \\
\text { qtr } \\
\# 2\end{array}$ & 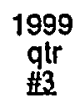 & $\begin{array}{c}1999 \\
\text { qtr } \\
\# 4\end{array}$ \\
\hline N/A & N/A & N/A & N/A & 32 \\
\hline 72 & 71 & N/A & N/A & 73 \\
\hline 79 & 80 & 79 & 80 & 81 \\
\hline 93 & 98 & 95 & 96 & 75 \\
\hline 75 & 78 & 78 & 78 & 82 \\
\hline 100 & 100. & 100 & 100 & 100 \\
\hline 39 & 32 & 32 & 32 & 36 \\
\hline 98 & 89 & 99 & 99 & 87 \\
\hline 97 & 100 & 100 & 100 & 98 \\
\hline 110 & 108 & 108 & 108 & 109 \\
\hline 110 & 109 & 108 & 108 & 109 \\
\hline 99 & 99 & 99 & 99 & 99 \\
\hline 100 & 100 & 100 & 100 & 100 \\
\hline 100 & 100 & 100 & 100 & 100 \\
\hline 100 & 100 & 100 & 100 & 84 \\
\hline 100 & 99 & 100 & 100 & 100 \\
\hline 99 & 99 & 99 & 99 & 81 \\
\hline 96 & 100 & 100 & 100 & 88 \\
\hline 96 & 100 & 100 & 100 & 88 \\
\hline 100 & 100 & 100 & 100 & 77 \\
\hline 99 & 98 & 99 & 99 & 86 \\
\hline 99 & 101 & N/A & $N / A$ & 100 \\
\hline 102 & 90 & 98 & 88 & 89 \\
\hline 78 & 74 & 79 & 80 & 78 \\
\hline 55 & 81 & 49 & 49 & 71 \\
\hline 96 & 96 & 96 & 96 & 79 \\
\hline 97 & 100 & 100 & 100 & 88 \\
\hline 97 & 100 & 100 & 100 & 88 \\
\hline 102 & 102 & 102 & 102 & 122 \\
\hline 99 & 99 & 99 & 99 & 90 \\
\hline 83 & 82 & .67 & 79 & 81 \\
\hline 82 & 72 & 79 & 69 & 82 \\
\hline 99 & 99 & 106 & 105 & 96 \\
\hline 95 & 93 & 94 & 89 & 99 \\
\hline N/A & N/A & $N / A$ & N/A & 97 \\
\hline
\end{tabular}

WEIGHTED AVERAGE PRICE (\$MMBTU) OF IMPORTS

\begin{tabular}{|c|c|c|c|c|}
\hline $\begin{array}{c}1998 \\
\text { qtr } \\
\# 4\end{array}$ & $\begin{array}{c}1999 \\
\text { qtr } \\
\mathbb{\# 1}\end{array}$ & $\begin{array}{c}1999 \\
\text { qtr } \\
\sharp 2\end{array}$ & $\begin{array}{c}1999 \\
\text { qtr } \\
\mathbb{1 3}\end{array}$ & $\begin{array}{c}1999 \\
\text { qtr } \\
\# 4\end{array}$ \\
\hline N/A & N/A & N/A & N/A & 2.66 \\
\hline 2.05 & 2.05 & N/A & N/A & 2.45 \\
\hline 1.91 & 1.87 & 1.89 & 2.16 & 2.38 \\
\hline 1.78 & 1.66 & 1.83 & 2.16 & 2.34 \\
\hline 1.76 & 1.65 & 1.65 & 2.14 & 2.44 \\
\hline 3.66 & 2.43 & 2.37 & 3.03 & 3.36 \\
\hline 1.19 & 1.19 & 1.31 & 1.21 & 1.13 \\
\hline 2.28 & 1.93 & 2.31 & 2.79 & 2.73 \\
\hline 2.18 & 2.01 & 2.14 & 2.49 & 2.91 \\
\hline 2.42 & 1.93 & 2.60 & 3.07 & 3.09 \\
\hline 2.37 & 1.87 & 2.55 & 3.02 & 3.04 \\
\hline 2.18 & 1.88 & 2.34 & 2.80 & 2.98 \\
\hline 2.32 & 1.93 & 2.31 & 2.79 & 2.79 \\
\hline 2.32 & 1.93 & 2.31 & 2.79 & 2.79 \\
\hline 2.18 & 1.89 & 2.36 & 2.85 & 2.99 \\
\hline 2.09 & 1.89 & 2.36 & 2.85 & 3.05 \\
\hline 2.18 & 1.88 & 2.34 & 2.80 & 2.91 \\
\hline 2.32 & 1.93 & 2.31 & 2.79 & 2.73 \\
\hline 2.32 & 1.93 & 2.31 & 2.79 & 2.73 \\
\hline 2.18 & 1.89 & 2.36 & 2.85 & 2.96 \\
\hline 2.09 & 1.89 & 2.36 & $2.85^{\circ}$ & 3.00 \\
\hline 2.11 & 2.10 & N/A & N/A & 2.77 \\
\hline 2.08 & 2.43 & 1.90 & 2.37 & 2.67 \\
\hline 2.02 & 2.16 & 1.93 & 2.07 & 2.52 \\
\hline 2.20 & 2.14 & 1.79 & 2.22 & 2.72 \\
\hline 2.18 & 1.88 & 2.34 & 2.80 & 2.91 \\
\hline 2.32 & 1.93 & 2,31 & 2.79 & 2.73 \\
\hline 2.32 & 1.93 & 2.31 & 2.79 & 2.73 \\
\hline 2.18 & 1.89 & 2.36 & 2.85 & 3.11 \\
\hline 2.09 & 1.89 & 2.36 & 2.85 & 3.01 \\
\hline 2.40 & 2.46 & 2.53 & 2.46 & 2.55 \\
\hline 1.73 & 1.55 & 1.66 & 1.99 & 2.26 \\
\hline 3.01 & 3.21 & 3.24 & 3.23 & 3.44 \\
\hline 1.83 & 2.02 & 2.02 & 1.93 & 1.91 \\
\hline N/A & N/A & N/A & N/A & 2.61 \\
\hline
\end{tabular}


LONG-TERM IMPORTS

ACTUAL IMPORTS AS A PERCENT OF AUTHORIZED VOLUMES

IMPORTER/EXPORTER

CITY OF GLENDALE, CALIFORNIAVEngage Energy Canada, L.P.

CITY OF PASADENA, CALIFORNIAVEngage Energy Canada

COENERGY TRADING COMPANYNarious Suppliers ।

COLONIAL NATURAL GAS/Alberta NE Gas (TCGS Ltd. 1)

COMMONWEALTH GAS COMPANY/Alberta NE Gas (TCGS Ltd. 1)

CONNECTICUT NATURAL GAS/Alberta NE Gas (AEC Oil \& Gas Company)

CONNECTICUT NATURAL GAS/AIberta NE Gas (TCGS Ltd. 1)

CONNECTICUT NATURAL GAS/AIberta NE Gas (TCGS Ltd. 2)

CONNECTICUT NATURAL GAS/Alberta Northeast Gas (ProGas)

CONNECTICUT NATURAL GAS/Alberta Northeast Gas (Producers Marketing)

CONSOLIDATED EDISON COMPANY OF N.Y./Alberta NE Gas (AEC Oil \& Gas)

CONSOLIDATED EDISON COMPANY OF N.Y./Alberta NE Gas (ProGas)

CONSOLIDATED EDISON COMPANY OF N.Y./Alberta NE Gas (Producers Marketing) 100 CONSOLIDATED EDISON COMPANY OF N.Y./Alberta NE Gas (TCGS Ltd. 1)

CONSOLIDATED EDISON COMPANY OF N.Y./Alberta NE Gas (TCGS Lid. 2)

CONSOLIDATED EDISON COMPANY OF N.Y./Amoco Canada

CORAL ENERGY RESOURCES, L.P.-ENRON GAS MARKET/Shell Canada Limiled CORAL ENERGY RESOURCES, L.P.-MIDAMERICAN ENER/Shell Canada Limited

CRESTAR ENERGY MARKETING CORPORATION/Crestar Energy

CRESTAR ENERGY MARKETING CORPORATION/Crestar Energy

DARTMOUTH POWER ASSOCIATES, L.P./Anderson Exploration Lid.

DARTMOUTH POWER ASSOCIATES, L.P./Crestar Energy Inc.

DARTMOUTH POWER ASSOCIATES, L.P./Direct Energy Marketing Ltd.

DARTMOUTH POWER ASSOCIATES, L.P./Remington Energy Ltd.

DISTRIGAS CORPORATION (88-37-LNG)/Sonatrach

DISTRIGAS CORPORATION (89-16-LNG)/Sonatrach

DISTRIGAS CORPORATION (95-100-LNG)/Cabot LNG Trading Limited

DUKE ENERGY LNG SALES, INC./Sonatrading

ELIZABETHTOWN GAS COMPANY/TCGS Ltd.

ENCOGEN NORTHWEST, L.P./CanWest Gas Supply, Inc.

ENERGYNORTH, INC./Alberta NE Gas (TCGS Ltd. 1)

ENGAGE ENERGY CANADA, L.P. - CIBOLA CORP./Engage Energy Canada, L.P. $\quad \begin{array}{r}97 \\ 103\end{array}$

ENGAGE ENERGY CANADA, L.P. - I'STATE POWER CO/Engage Energy Canada, L.P. 85

ENGAGE ENERGY CANADA, L.P. - METRO. UTIL. DIS/Engago Energy Canada, L.P. 82

ENGAGE ENERGY CANADA, L.P. - MIDAMERICAN/Engage Energy Canada, L.P. $\quad 103$

\begin{tabular}{|c|c|c|c|c|}
\hline $\begin{array}{l}1998 \\
\text { gtr } \\
\# 4\end{array}$ & $\begin{array}{l}1999 \\
\text { qtr } \\
\text { 此 }\end{array}$ & 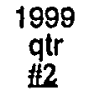 & 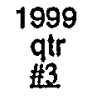 & 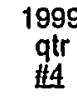 \\
\hline 91 & 98 & 95 & 99 & 101 \\
\hline 98 & 98 & 97 & 96 & 98 \\
\hline N/A & 29 & 41 & 83 & 146 \\
\hline 96 & 100 & 100 & 100 & 88 \\
\hline 100 & 100 & 100 & 100 & 88 \\
\hline 98 & 101 & 101 & 101 & 88 \\
\hline 95 & 100 & 100 & 100 & 88 \\
\hline 94 & 99 & 99 & 99 & 87 \\
\hline 98 & 100 & 100 & 100 & 78 \\
\hline 99 & 100 & 101 & 101 & 89 \\
\hline 101 & 101 & 101 & 100 & 83 \\
\hline 100 & 100 & 100 & 100 & 78 \\
\hline 100 & 100 & 100 & 100 & 91 \\
\hline 97 & 100 & 100 & 100 & 88 \\
\hline 97 & 100 & 100 & 100 & 88 \\
\hline 97 & 100 & 101 & 100 & 101 \\
\hline N/A & 99 & 102 & 101 & 101 \\
\hline N/A & 99 & 98 & 100 & 95 \\
\hline 96 & 98 & 83 & 79 & 88 \\
\hline 96 & 99 & 101 & 101 & 95 \\
\hline 123 & 122 & 121 & 117 & 72 \\
\hline 85 & 78 & 61 & 127 & 70 \\
\hline$N / A$ & N/A & N/A & N/A & N/A \\
\hline 57 & 70 & 89 & 80 & 64 \\
\hline N/A & N/A & N/A & N/A & N/A \\
\hline N/A & N/A & N/A & N/A & N/A \\
\hline N/A & $N / A$ & N/A & N/A & N/A \\
\hline 60 & 58 & 60 & 63 & 65 \\
\hline 80 & 86 & 100 & 100 & 100 \\
\hline 80 & 82 & 59 & 78 & $N / A$ \\
\hline 97 & 100 & 100 & 100 & 91 \\
\hline 103 & 104 & 103 & 103 & 102 \\
\hline 85 & 110 & 109 & 109 & 110 \\
\hline 82 & 103 & 102 & 102 & 74 \\
\hline 103 & 103 & 101 & 103 & 82 \\
\hline
\end{tabular}

WEIGHTED AVERAGE PAICE (\$MMBTU) OFIMPORTS

\begin{tabular}{|c|c|c|c|c|}
\hline $\begin{array}{c}1998 \\
\text { qtr } \\
\# 4\end{array}$ & 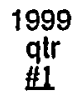 & $\begin{array}{c}1999 \\
\text { qtr } \\
\mathbb{2} 2\end{array}$ & 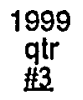 & $\begin{array}{c}1999 \\
\text { qtr } \\
\# 4\end{array}$ \\
\hline 2.01 & 2.03 & 2.02 & 1.91 & 1.64 \\
\hline 2.00 & 2.02 & 2.00 & 1.89 & 1.90 \\
\hline N/A & 1.98 & 2.41 & 2.71 & 2.75 \\
\hline 2.32 & 1.93 & 2.31 & 2.79 & 2.73 \\
\hline 2.32 & 1.93 & 2.31 & 2.79 & 2.73 \\
\hline 2.18 & 1.88 & 2.34 & 2.80 & 2.93 \\
\hline 2.32 & 1.93 & 2.31 & 2.79 & 2.73 \\
\hline 2.32 & 1.93 & 2.31 & 2.79 & 2.73 \\
\hline 2.18 & 1.89 & 2.36 & 2.85 & 2.96 \\
\hline 2.10 & 1.89 & 2.36 & 2.85 & 3.01 \\
\hline 2.18 & 1.88 & 2.34 & 2.81 & 2.91 \\
\hline 2.18 & 1.89 & 2.36 & 2.85 & 2.96 \\
\hline 2.09 & 1.89 & 2.36 & 2.85 & 3.01 \\
\hline 2.32 & 1.93 & 2.31 & 2.79 & 2.73 \\
\hline 2.32 & 1.93 & 2.31 & 2.79 & 2.73 \\
\hline 2.20 & 2.23 & 2.23 & 2.68 & 3.03 \\
\hline N/A & 1.48 & 1.73 & 2.09 & 2.26 \\
\hline N/A & 1.49 & 1.73 & 2.09 & 2.22 \\
\hline 1.99 & 1.93 & 2.32 & 2.87 & 2.86 \\
\hline 1.94 & 1.88 & 2.21 & 2.66 & 2.65 \\
\hline 2.45 & 2.51 & 2.62 & 2.84 & 3.19 \\
\hline 2.54 & 2.66 & 2.52 & 3.62 & 3.58 \\
\hline 2.30 & N/A & N/A & N/A & N/A \\
\hline 2.44 & 2.36 & 2.61 & 3.66 & 3.71 \\
\hline 2.34 & 2.35 & 2.18 & 2.36 & 2.61 \\
\hline N/A & 2.32 & 2.02, & N/A & $N / A$ \\
\hline$N / A$ & N/A & 1.85 & 2.24 & 2.73 \\
\hline 2.00 & 2.00 & 2.00 & 2.00 & 2.00 \\
\hline 3.15 & 3.08 & 2.57 & 3.01 & 3.12 \\
\hline 2.35 & 2.35 & 2.35 & 2.35 & N/A \\
\hline 2.32 & 1.93 & 2.31 & 2.79 & 2.75 \\
\hline 1.87 & 1.60 & 1.87 & 2.33 & 2.39 \\
\hline 1.97 & 1.72 & 2.02 & 2.33 & 2.35 \\
\hline 1.96 & 1.69 & 2.00 & 2.46 & 2.20 \\
\hline 1.81 & 1.60 & 1.88 & 2.28 & 2.14 \\
\hline
\end{tabular}




\section{LONG-TERM IMPORTS}

\section{ACTUALIMPORTS AS A PERCENT OFAUTHORIZED VOLUMES}

\section{IMPORTER/EXPORTER}

ENGAGE ENERGY CANADA, L.P. - N'WESTERN PUB./Engage Energy Canada, L.P. $\quad 85$ ENGAGE ENERGY CANADA, L.P. - WISCONSIN GAS/Engage Enengy Canada, L.P. 103 ENGAGE ENERGY U.S., L.P./Engage Energy Canada, L.P.

ENGAGE ENERGY U.S., L.P.Morgan Hydrocarbons Inc.

ENGAGE ENERGY U.S., L.P.Morrison Petroleums Lid.

ENGAGE ENERGY U.S., L.P./Petro-Canada Resources

ENGAGE ENERGY U.S., L.P./Rio Alto, Pinnacle, Jordan

ENGAGE ENERGY U.S., L.P.Narious Suppliers

ENGAGE ENERGY U.S., L.P.Narious Suppliers

ENGAGE ENERGY U.S., L.P.Narious Suppliers

ENRON NORTH AMERICA CORP.JECTR - Canada

ENRON NORTH AMERICA CORP.IECTR - Canada

ENPON NORTH AMERICA CORP.IECTR - Canada

ENRON NORTH AMERICA CORP./ECTR - Canada

ENRON NORTH AMERICA CORP.IECTR - Canada

ESSEX COUNTY GAS CO./Alberta NE Gas (TCGS Ltd. 1)

FULTON COGENERATION ASSOCIATES/Star Oil \& Gas Limited

GRANITE STATE GAS TRANSMISSION, INC./Direct Energy Marketing Ltd.

GRANITE STATE GAS TRANSMISSION, INC./Shell Canada Limited

GRANITE STATE GAS TRANSMISSION, INC./Shell Canada Limited

HERMISTON GENERATING COMPANY, L.P./CanStates Gas Marketing

HERMISTON GENERATING COMPANY, L.P./Home Oil Company Limited

HUSKY GAS MARKETING INC./Husky Oil Operations Ltd.

IGI RESOURCES, INC.Mobil Oil Canada

LOCKPORT ENERGY ASSOCIATES, L.P./ProGas Limited

MIDLAND COGENERATION VENTURE1/Union Pacific Resources

MIDLAND COGENERATION VENTURE2/Coral Energy

MIDLAND COGENERATION VENTURE3/Husky Oil

MIDLAND COGENERATION VENTURE4/TransCanada Pipelines Limited

MIDLAND COGENERATION VENTURE5/Poco Potroleums Litd.

MIDLAND COGENERATION VENTURE6/Union Pacilic Resources

MINNEGASCO, INC./TransCanada Pipelines Limited

N.Y. STATE ELECTRIC \& GAS CO./Alberta NE Gas (AEC Oil \& Gas Co.)

N.Y. STATE ELECTRIC \& GAS CO./Alberta NE Gas (TCGS Ltd. 2)

N.Y. STATE ELECTRIC \& GAS CO./Alberta Northeast Gas (ProGas)

\begin{tabular}{|c|c|c|c|c|}
\hline $\begin{array}{c}1998 \\
\text { qtr } \\
\# 4\end{array}$ & $\begin{array}{c}1999 \\
\text { qtr } \\
\# 1\end{array}$ & $\begin{array}{c}1999 \\
\text { qtr } \\
\# 2\end{array}$ & $\begin{array}{c}1999 \\
\text { qtr } \\
\# 3\end{array}$ & $\begin{array}{c}1999 \\
\text { qtr } \\
\# 4\end{array}$ \\
\hline 85 & 106 & 108 & 107 & 83 \\
\hline 103 & 104 & 103 & 103 & 104 \\
\hline 22 & 23 & 29 & 21 & 17 \\
\hline 98 & 111 & 102 & 102 & 102 \\
\hline 101 & 102 & 102 & 102 & 102 \\
\hline 101 & 101 & 101 & 101 & 101 \\
\hline 53 & 70 & 65 & 65 & 65 \\
\hline 85 & 105 & 61 & 61 & 61 \\
\hline 39 & 31 & 62 & 90 & 96 \\
\hline 94 & 73 & 98 & 98 & 100 \\
\hline 47 & 13 & 13 & 13 & 13 \\
\hline 100 & 99 & N/A & N/A & N/A \\
\hline 102 & 98 & 101 & 101 & 101 \\
\hline 11 & 95 & 95 & 95 & 95 \\
\hline 100 & 95 & 103 & 104 & 104 \\
\hline 100 & 100 & 100 & 100 & 88 \\
\hline 92 & N/A & N/A & N/A & N/A \\
\hline 102 & 102 & 100 & 100 & 100 \\
\hline 100 & 100 & N/A & N/A & N/A \\
\hline 101 & 101 & 101 & 101 & 101 \\
\hline 90 & 90 & 73 & 88 & 90 \\
\hline 90 & 90 & 73 & 72 & 72 \\
\hline 91 & 83 & 85 & 84 & 85 \\
\hline N/A & N/A & 0 & N/A & N/A \\
\hline 72 & 56 & 65 & 87 & 60 \\
\hline 100 & 100 & 100 & 100 & 100 \\
\hline 100 & 100 & 100 & 100 & 100 \\
\hline 100 & 100 & 100 & 100 & 100 \\
\hline 100 & 100 & 100 & 100 & 100 \\
\hline 83 & 100 & 83 & 100 & 100 \\
\hline 100 & 100 & 52 & N/A & 100 \\
\hline 95 & 101 & 62 & 62 & 62 \\
\hline 102 & 102 & 102 & 102 & 84 \\
\hline 95 & 99 & 99 & 99 & 87 \\
\hline 101 & 101 & 101 & 101 & 79 \\
\hline
\end{tabular}

WEIGHTED AVERAGE PRICE (\$MMBTU) OEIMPORTS

$\begin{array}{ccccc}\begin{array}{c}1998 \\ \text { qtr }\end{array} & \begin{array}{c}1999 \\ \text { qtr }\end{array} & \begin{array}{c}1999 \\ \text { qtr }\end{array} & \begin{array}{c}1999 \\ \text { qtr }\end{array} & \begin{array}{c}1999 \\ \text { qtr }\end{array} \\ \# & \text { \#1 } & \# 2 & \text { \#3 } & \text { \#4 } \\ & & & & \\ 1.98 & 1.69 & 2.00 & 2.43 & 2.24 \\ 1.87 & 1.62 & 1.93 & 2.36 & 2.29 \\ 2.45 & 2.23 & 2.33 & 2.50 & 2.95 \\ 2.30 & 2.07 & 2.33 & 2.77 & 2.92 \\ 1.91 & 1.67 & 1.94 & 2.33 & 2.13 \\ 1.91 & 1.67 & 1.94 & 2.33 & 2.13 \\ 2.37 & 2.09 & 2.32 & 2.77 & 2.92 \\ 1.97 & 1.85 & 2.24 & 2.68 & 2.74 \\ 1.95 & 1.86 & 2.37 & 2.66 & 2.93 \\ 1.67 & 1.58 & 2.14 & 2.56 & 2.62 \\ 2.00 & 1.72 & 2.04 & 2.47 & 2.48 \\ 2.26 & 1.87 & \text { N/A } & \text { N/A } & \text { N/A } \\ 2.32 & 1.85 & 2.23 & 2.67 & 2.73 \\ 2.06 & 1.63 & 2.01 & 2.54 & 2.59 \\ 2.28 & 1.85 & 2.24 & 2.67 & 2.69 \\ 2.32 & 1.93 & 2.31 & 2.79 & 2.73 \\ 2.85 & \text { N/A } & \text { N/A } & \text { N/A } & \text { N/A } \\ 2.48 & 2.52 & 2.51 & 2.49 & 2.34 \\ 2.72 & 2.24 & \text { N/A } & \text { N/A } & \text { N/A } \\ 2.35 & 2.05 & 2.26 & 2.60 & 2.86 \\ 2.42 & 2.47 & 2.55 & 2.48 & 2.56 \\ 2.42 & 2.47 & 2.55 & 2.48 & 2.56 \\ 1.62 & 1.48 & 1.76 & 2.15 & 3.04 \\ \text { N/A } & \text { N/A } & \text { N/A } & \text { N/A } & \text { N/A } \\ 4.12 & 4.70 & 4.34 & 3.93 & 4.66 \\ 1.72 & 1.72 & 1.68 & 1.66 & 1.65 \\ 1.65 & 1.65 & 1.63 & 1.58 & 1.57 \\ 1.90 & 1.90 & 1.90 & 1.90 & 1.90 \\ 1.64 & 1.64 & 1.59 & 1.57 & 1.56 \\ 2.49 & 2.44 & 2.47 & 2.49 & 2.52 \\ 2.87 & 2.90 & 2.92 & \text { N/A } & 2.99 \\ 2.13 & 1.61 & 1.90 & 2.37 & 2.40 \\ 2.18 & 1.88 & 2.34 & 2.80 & 2.91 \\ 2.32 & 1.93 & 2.31 & 2.79 & 2.73 \\ 2.18 & 1.89 & 2.36 & 2.85 & 2.96\end{array}$


Page - 4

\section{LONG-TERM IMPORTS}

ACTUAL MMPORTS AS A PERCENT OF AUTHORIZED VOLUMES

N.Y. STATE ELECTRIC \& GAS CO./Alberta Northeast Gas (Producers Markeling) N.Y. STATE ELECTRIC \& GAS CO./Crestar Energy

N.Y. STATE ELECTRIC \& GAS CO./Progas Limited

NATIONAL FUEL GAS DISTAIBUTION CORP./Alberta NE Gas (TCGS Ltd.)

NATIONAL STEEL CORPORATION/Direct Energy Marketing, Ltd.

NEW JERSEY NATURAL GAS COMPANY/Alberta NE Gas (AEC Oil \& Gas Company) 100

NEW JERSEY NATÜRAL GAS COMPANY/Alberta NE Gas (TCGS Ltd. 1)

NEW JERSEY NATURAL GAS COMPANY/Alberta NE Gas (TCGS Ltd. 2)

NEW JERSEY NATURAL GAS COMPANY/Alberta Northeast Gas (ProGas)

NEW JERSEY NATURAL GAS COMPANY/Alberta Northeast Gas (Producers Mklg.)

NIAGARA MOHAWK POWER CORPORATION/TCGS Ltd.

NORTH JERSEY ENERGY ASSOCIATES/ProGas Limited

NORTHEAST ENERGY ASSOCIATES/ProGas Limited

NORTHERN MINNESOTA UTILITIESTTCGS LId.

NORTHERN MINNESOTA UTILITIES - EASTERN MARKET/TCGS Ltd.

NORTHERN MINNESOTA UTILITIES - WESTERN MARKET/TCGS LId.

NORTHERN STATES POWER COMPANY/Amoco Canada (WI)

NORTHERN STATES POWER COMPANY/Canadian Occidental (WI)

NORTHERN STATES POWER COMPANY/ProGas Limited (WI)

NORTHERN STATES POWER COMPANY (MINNESOTA)/Amoco Canada (MN)

NORTHERN STATES POWER COMPANY (MINNESOTA)/TCGS Lid.

NORTHERN UTILITIES, INC./Renaissance Energy Ltd.

NORTHWEST NATURAL GAS COMPANYIAmoco Canada

NORTHWEST NATURAL GAS COMPANY/CanWest Gas Supply

NORTHWEST NATURAL GAS COMPANYIEngage Energy Canada

NORTHWEST NATURAL GAS COMPANY/Poco Petroleums Limited

NORTHWEST NATURAL GAS COMPANY/Summit Resources Limited

NUI CORPORATION/TCGS LId.

NW ALASKAN PIPELINE CO-Pan-Alberta Gas (l)/Pan-Alberta Gas Ltd.

NW ALASKAN PIPELINE CO-Pan-Alberta Gas (II)/Pan-Alberta Gas Ltd.

OCEAN STATE POWER/ProGas Limited

OCEAN STATE POWER II/ProGas Limited

OCEAN STATE POWER IITCGS L.td.

ORANGE AND ROCKLAND UTILITIES, INC.Mascana Energy Marketing

ORCHARD GAS CORPORATION/ProGas Limiled

\begin{tabular}{rrrrr}
1998 & 1999 & 1999 & 1999 & 1999 \\
qlr & \multicolumn{1}{c}{ qtr } & \multicolumn{1}{c}{ qtr } & \multicolumn{1}{c}{ qtr } & \multicolumn{1}{c}{ qtr } \\
\#4 & $\$ 11$ & $\$ 2$ & $\$ 3$ & 14 \\
& & & & \\
99 & 99 & 99 & 99 & 90 \\
70 & 67 & 98 & 38 & 79 \\
88 & 90 & 93 & 90 & 86 \\
97 & 100 & 100 & 100 & 91 \\
140 & 129 & 101 & 95 & 128 \\
102 & 102 & 102 & 102 & 90 \\
96 & 100 & 100 & 100 & 88 \\
95 & 99 & 99 & 99 & 87 \\
100 & 100 & 100 & 100 & 81 \\
99 & 99 & 99 & 99 & 87 \\
100 & 100 & 100 & 100 & 101 \\
87 & 91 & 90 & 90 & 90 \\
97 & 103 & 92 & 97 & 100 \\
100 & 100 & 100 & 100 & 100 \\
57 & 59 & 15 & 3 & 7 \\
92 & 132 & 27 & 24 & 83 \\
78 & 83 & 39 & 40 & 61 \\
83 & 100 & 100 & 99 & 112 \\
90 & 96 & 100 & 97 & 94 \\
85 & 99 & 96 & 99 & 94 \\
78 & 99 & 100 & 100 & 91 \\
99 & 101 & 99 & 99 & 99 \\
102 & 103 & 103 & 103 & 103 \\
103 & 101 & 104 & 102 & 103 \\
59 & 59 & 58 & 59 & 59 \\
98 & 98 & 69 & 69 & 99 \\
97 & 98 & 62 & 63 & 98 \\
76 & 100 & 93 & 100 & 94 \\
99 & 97 & 99 & 101 & 94 \\
88 & 79 & 79 & 80 & 78 \\
100 & 100 & 100 & 100 & 100 \\
100 & 100 & 100 & 100 & 100 \\
100 & 100 & 100 & 100 & 100 \\
100 & 101 & 101 & 100 & 100 \\
94 & 99 & 100 & 100 & 100 \\
& & & &
\end{tabular}

WEIGHTED AVERAGE PRICE (\$MMBTU) OFIMPORTS

\begin{tabular}{|c|c|c|c|c|}
\hline $\begin{array}{c}1998 \\
\text { qtr } \\
\text { 柆4 }\end{array}$ & $\begin{array}{c}1999 \\
\text { qtr } \\
\text { \#1 }\end{array}$ & $\begin{array}{c}1999 \\
\text { qtr } \\
\mathbb{\# 2}\end{array}$ & $\begin{array}{c}1999 \\
\text { qtr } \\
\# 3\end{array}$ & $\begin{array}{c}199 \\
\text { qtr } \\
\# 4\end{array}$ \\
\hline 2.09 & 1.89 & 2.36 & 2.85 & 3.0 \\
\hline 3.39 & 3.80 & 3.46 & 4.98 & \\
\hline 2.11 & 2.31 & 2.46 & 2.79 & \\
\hline 2.31 & 1.94 & 2.31 & 2.79 & \\
\hline 2.49 & 2.57 & 2.38 & 2.38 & \\
\hline 2.18 & 1.88 & 2.34 & 2.80 & \\
\hline 2.32 & 1.93 & 2.31 & 2.79 & 2.7 \\
\hline 2.32 & 1.93 & 2.31 & 2.79 & 2.7 \\
\hline 2.18 & 1.89 & 2.36 & 2.85 & 2.97 \\
\hline 2.09 & 1.89 & 2.36 & 2.85 & 3.0 \\
\hline 2.51 & 2.17 & 2.60 & 3.05 & 2.98 \\
\hline 3.37 & 3.24 & 3.01 & 2.97 & 2.97 \\
\hline 3.65 & 3.61 & 3.71 & 3.63 & 3.60 \\
\hline 1.85 & 1.47 & 1.85 & 2.32 & 2.36 \\
\hline 2.17 & 1.93 & 1.98 & 2.38 & $2.4 E$ \\
\hline 2.00 & 1.74 & 1.93 & 2.44 & 2.50 \\
\hline 2.80 & 2.35 & 2.80 & 2.78 & 2.37 \\
\hline 2.45 & 2.10 & 2.37 & 3.01 & 3.18 \\
\hline 2.32 & 2.11 & 2.34 & 2.80 & 2.88 \\
\hline 2.22 & 1.93 & 2.35 & 2.76 & 2.7 \\
\hline 2.31 & 1.93 & 2.18 & 2.65 & $2.7 \mathrm{~s}$ \\
\hline 3.66 & 2.67 & 2.30 & 3.03 & 3.34 \\
\hline 1.78 & 1.80 & 1.80 & 1.80 & 2.47 \\
\hline 1.99 & 2.10 & 1.81 & 2.25 & 2.56 \\
\hline 2.03 & 2.17 & 2.08 & 2.22 & 2.64 \\
\hline 2.09 & 2.11 & 2.22 & 2.22 & 2.57 \\
\hline 1.88 & 1.98 & 2.04 & 2.04 & 2.61 \\
\hline 2.76 & 2.28 & 2.80 & 3.19 & 3.17 \\
\hline 1.94 & 1.73 & 1.99 & 2.30 & 2.38 \\
\hline 1.70 & 1.44 & 1.70 & 2.07 & 2.16 \\
\hline 2.12 & 1.95 & 1.83 & 1.80 & 1.80 \\
\hline 2.12 & 1.95 & 1.83 & 1.80 & 1.80 \\
\hline 2.11 & 1.95 & 1.83 & 1.80 & 1.81 \\
\hline 2.88 & 3.06 & 2.78 & 3.29 & 3.47 \\
\hline 2.69 & 2.64 & 2.54 & 2.68 & 3.27 \\
\hline
\end{tabular}




\section{LONG-TERM IMPORTS}

\section{ACTUAL IMPORTS AS A PERCENT OF AUTHORIZED VOLUMES}

IMPORTER/EXPORTER

PAN-ALBERTA GAS (U.S.) INC./Pan-Alberta Gas Ltd.

PAWTUCKET POWER ASSOCIATES/Anderson Exploration Ltd.

PAWTUCKET POWER ASSOCIATES/Tarragon Oil \& Gas Limited

PG ENERGY/TransCanada PipeLines Limited

PITTSFIELD GENERATING CO., L.P.IAnderson Exploration Ltd.

PITTSFIELD GENERATING CO., L.P.TTalisman Energy Inc.

POCO MARKETING LTD./IGI Resources, Inc.

PORTAL MUNICIPAL GAS/Sask Energy Inc.

PROGAS U.S.A. INC./ProGas Limited

PROGAS U.S.A. INC.IProGas Limited

PROGAS U.S.A. INC.IProGas Limited

PROGAS U.S.A. INC.IProGas Limited

PROGAS U.S.A. INC.IProGas Limited

PROGAS U.S.A. INC. - (DYNEGY)/ProGas Ltd.

PROGAS U.S.A. INC. - (TENASKA GAS COMPANY)/ProGas Limited

PROGAS U.S.A., INC/Progas U.S.A. Inc.

PROGAS U.S.A., INC/Progas U.S.A. Inc.

PROJECT ORANGE ASSOCIATES, L.P./Noranda, Inc.

PUBLIC SERVICE ELECTRIC \& GAS/Aiberta NE Gas (TCGS Ltd. 1)

PUGET SOUND ENERGY, INC.IAmoco Canada

PUGET SOUND ENERGY, INC.IDuke Energy

PUGET SOUND ENERGY, INC./Engage Energy Canada

PUGET SOUND ENERGY, INC./Engage Energy Canada

PUGET SOUND ENERGY, INC./Poco Petroleums Ltd.

RENAISSANCE ENERGY (U.S.) INC./Renaissance Energy Limited

RENAISSANCE ENERGY (U.S.) INC./Renaissance Energy Limited

RENAISSANCE ENERGY (U.S.) INC./Renaissance Energy Limited

ROCHESTER GAS AND ELECTRIC CORPORATION/Renaissance Energy Limited

ROCK-TENN COMPANY, MILL DIV., INC.Nascana Energy Inc.

SAN DIEGO GAS \& ELECTRIC COMPANY/Bow Valley Industries Ltd.

SAN DIEGO GAS \& ELECTRIC COMPANY/Canadian Hunter Marketing Lid.

SAN DIEGO GAS \& ELECTRIC COMPANYISummit Resources Ltd.

SARANAC POWER PARTNERS, L.P./Shell Canada Limited

SELKIRK COGEN PARTNERS, L.P./Imperial Oil Resources Limited

SELKIRK COGEN PARTNERS, L.P./PanCanadian Petroleum Ltd.

\begin{tabular}{|c|c|c|c|c|}
\hline $\begin{array}{c}1998 \\
\text { qtr } \\
\# 4\end{array}$ & $\begin{array}{c}1999 \\
\text { qtr } \\
\# 1\end{array}$ & $\begin{array}{c}1999 \\
\text { qtr } \\
\# 2\end{array}$ & 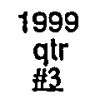 & 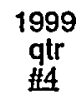 \\
\hline 88 & 91 & 77 & 90 & 99 \\
\hline 89 & 65 & 88 & 82 & 84 \\
\hline 85 & 63 & 85 & 83 & 81 \\
\hline 102 & 102 & 102 & N/A & N/A \\
\hline 92 & 92 & 91 & 99 & 101 \\
\hline 92 & 91 & 92 & 100 & 102 \\
\hline 99 & 99 & 99 & 99 & 99 \\
\hline 2 & 3 & 1 & 0 & 2 \\
\hline 100 & 100 & 100 & 100 & 100 \\
\hline 67 & 51 & 73 & 72 & 98 \\
\hline 84 & 101 & 57 & 50 & 97 \\
\hline 96 & 93 & 72 & 56 & 56 \\
\hline N/A & 85 & 81 & 81 & 81 \\
\hline 93 & 96 & 96 & 97 & 98 \\
\hline 114 & 96 & 97 & 97 & 78 \\
\hline 72 & 98 & 76 & 100 & 99 \\
\hline 92 & 86 & 139 & 89 & 92 \\
\hline N/A & N/A & N/A & N/A & N/A \\
\hline 100 & 100 & 100 & 100 & 93 \\
\hline 104 & 104 & 104 & 104 & 100 \\
\hline 103 & 103 & 103 & 103 & 100 \\
\hline 104 & 104 & 104 & 104 & 119 \\
\hline 81 & 81 & 81 & 81 & 77 \\
\hline 104 & 104 & 104 & 104 & 102 \\
\hline 102 & 103 & 102 & 99 & 102 \\
\hline 100 & 100 & 100 & 100 & 100 \\
\hline 58 & 72 & N/A & N/A & 61 \\
\hline N/A & 0 & 22 & $N / A$ & N/A \\
\hline 98 & 106 & 84 & 85 & 97 \\
\hline N/A & N/A & 0 & N/A & N/A \\
\hline N/A & $N / A$ & 0 & N/A & N/A \\
\hline N/A & N/A & 0 & N/A & N/A \\
\hline 75 & 83 & 83 & 85 & 83 \\
\hline 95 & 100 & 106 & 100 & 100 \\
\hline 95 & 100 & 106 & 100 & 100 \\
\hline
\end{tabular}

WEIGHTED AVERAGE PRICE (\$MMBTU) OF IMPORTS

\begin{tabular}{|c|c|c|c|c|}
\hline $\begin{array}{c}1998 \\
\text { qtr } \\
\# 4\end{array}$ & $\begin{array}{c}1999 \\
\text { qtr } \\
\# 1\end{array}$ & 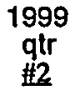 & 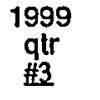 & $\begin{array}{c}1999 \\
\text { qtr } \\
\# 4\end{array}$ \\
\hline 1.25 & 1.64 & 1.24 & 1.73 & 1.97 \\
\hline 2.34 & 2.61 & 2.52 & 2.96 & 2.93 \\
\hline 2.38 & 2.62 & 2.63 & 3.13 & 3.19 \\
\hline 2.57 & 2.29 & 2.46 & N/A & N/A \\
\hline 2.21 & 2.16 & 2.34 & 2.59 & 2.72 \\
\hline 2.23 & 2.11 & 2.48 & 3.20 & 2.96 \\
\hline 1.98 & 2.10 & 1.84 & 2.27 & 2.44 \\
\hline 3.02 & 3.06 & 2.80 & 3.13 & 2.76 \\
\hline 2.44 & 2.56 & 2.27 & 2.22 & 2.44 \\
\hline 2.22 & 2.25 & 2.03 & 2.49 & 2.51 \\
\hline 2.56 & 2.63 & 2.38 & 2.89 & 2.87 \\
\hline 2.28 & 1.94 & 2.29 & 2.81 & 2.79 \\
\hline N/A & 1.60 & 2.06 & 2.52 & 2.58 \\
\hline 1.98 & 1.61 & 1.96 & 2.41 & 2.42 \\
\hline 1.97 & 1.60 & 1.95 & 2.41 & 2.42 \\
\hline 2.47 & 2.21 & 2.29 & 2.71 & 2.90 \\
\hline 2.10 & 1.90 & 2.16 & 2.35 & 2.70 \\
\hline 2.33 & 2.58 & 3.64 & 3.04 & 2.97 \\
\hline 2.32 & 1.93 & 2.31 & 2.79 & 2.77 \\
\hline 2.00 & 2.09 & 1.83 & 2.26 & 2.60 \\
\hline 2.01 & 2.09 & 1.83 & 2.26 & 2.60 \\
\hline 1.97 & 2.12 & 1.89 & 2.33 & 2.78 \\
\hline 1.92 & 1.88 & 1.91 & 2.21 & 2.50 \\
\hline 2.00 & 2.09 & 1.83 & 2.26 & 2.56 \\
\hline 1.59 & 1.69 & 1.71 & 2.02 & 2.28 \\
\hline 2.55 & 2.24 & 2.63 & 3.10 & 3.36 \\
\hline 1.95 & 1.90 & N/A & $N / A$ & 2.74 \\
\hline$N / A$ & N/A & 3.71 & N/A & N/A \\
\hline 2.77 & 2.46 & 3.81 & 4.49 & 3.33 \\
\hline$N / A$ & $N / A$ & N/A & N/A & N/A \\
\hline$N / A$ & N/A & N/A & N/A & N/A \\
\hline N/A & N/A & N/A & N/A & N/A \\
\hline 3.58 & 3.61 & 3.61 & 3.61 & 3.71 \\
\hline 2.49 & 2.20 & 2.43 & 2.46 & 2.61 \\
\hline 2.15 & 2.17 & 2.22 & 2.47 & 2.61 \\
\hline
\end{tabular}


Page - 6

\section{LONG-TERM IMPORTS}

ACTUAL IMPORTS AS A PERCENT OF AUTHORIZED VOLUMES

SELKIRK COGEN PARTNERS, L.P./Paramount Resources Ltd.

SELKIRK COGEN PARTNERS, L.P./Producers Marketing, Ltd.

SIERRA PACIFIC POWER COMPANYIAmoco Canada

SITHE/NDEPENDENCE POWER PARTNERS, L.P./Enron Power Services Inc. SOUTHEASTERN MICHIGAN GAS COMPANYITCGS LId.

SOUTHERN CONNECTICUT GAS/Alberta NE Gas (AEC Oil \& Gas Company)

SOUTHEAN CONNECTICUT GASIAlberta NE Gas (TCGS Ltd. 1)

SOUTHERN CONNECTICUT GAS/Alberta NE Gas (TCGS Ltd. 2)

SOUTHERN CONNECTICUT GAS/Alberta Northeast Gas (ProGas)

SOUTHERN CONNECTICUT GAS/Alberta Northeast Gas (Producers Marketing)

ST. LAWRENCE GAS COMPANY, INC./TCGS Ltd.

STATOIL ENERGY, INC./Talisman Energy Ine.

STATOIL ENERGY, INC./Talisman Energy Inc.

SUMAS COGENERATION COMPANY, L.P./ENCO Gas, Ltd.

TENASKA WASHINGTON PARTNERS, L.P./Puget Sound Energy, Inc.

THE U.S. GENERAL SERVICES ADMIN./Chief Mountain Natural Gas Coop.

TM STAR FUEL COMPANY/CanWest Gas Supply

TRANSCANADA GAS SERVICES INC./TransCanada Gas Services

TRANSCANADA GAS SERVICES INC./Yankee Gas Services

TRANSCO ENERGY MARKETING COMPANY/Canstates Gas Marketing

TRANSCO ENERGY MARKETING COMPANY-BG\&E/Canstates Gas Marketing

TRANSCO ENERGY MARKETING COMPANY-LILCO/Canstates Gas Marketing

TRANSCO ENERGY MARKETING COMPANY-PSE\&G/Canstates Gas Marketing

UNITED STATES GYPSUM COMPANYIHUsky Oil Operations Ltd.

UNITED STATES GYPSUM COMPANY/Renaissance Energy, L.td.

USGEN NEW ENGLAND, INC./Canadian Natural Resources

USGEN NEW ENGLAND, INC./Renaissance Energy Limited

UTILICORP UNITED, INC./ProGas Limiled

UTILICORP UNITED, INC./TCGS LId.

VALLEY GAS COMPANYIAlborta NE Gas (TCGS Ltd. 1)

VERMONT GAS SYSTEMS INC./TCGS LId.

VERMONT GAS SYSTEMS, INC./Renaissance Energy Lid.

VERMONT GAS SYSTEMS, INC.Narious Suppliers

WISCONSIN ELECTRIC POWER CO. (GAS OPS)/Progas Limited

WISCONSIN ELECTRIC POWER CO. (GAS OPS)/TCGS LId.
98

100

81

38

91

60

91

IA /A

\begin{tabular}{rrrrr}
1998 & 1999 & 1999 & 1999 & 1999 \\
qtr & qtr & $\begin{array}{c}\text { qtr } \\
\text { qtr }\end{array}$ & \multicolumn{1}{c}{ qtr } \\
\#1 & $\$ 2$ & $\# 3$ & $\# 4$ \\
& & & & \\
91 & 92 & 99 & 92 & 90 \\
94 & 99 & 106 & 99 & 99 \\
99 & 97 & 99 & 99 & 98 \\
87 & 88 & 88 & 86 & 88 \\
93 & 100 & 101 & 101 & 100 \\
99 & 99 & 99 & 99 & 81 \\
145 & 150 & 150 & 150 & 138 \\
N/A & N/A & 0 & N/A & N/A \\
100 & 100 & 100 & 100 & 80 \\
101 & 100 & 101 & 101 & 91 \\
54 & 74 & 29 & 13 & 60 \\
94 & 86 & 96 & 93 & 91 \\
92 & 90 & 82 & 93 & 93 \\
47 & 36 & 7 & 96 & 99 \\
N/A & N/A & N/A & 0 & N/A \\
0 & 1 & 1 & 0 & 1 \\
100 & 94 & 78 & 90 & 92 \\
N/A & 9 & 50 & 44 & 26 \\
N/A & N/A & N/A & N/A & 72 \\
102 & 107 & 107 & 107 & 84 \\
106 & 63 & N/A & N/A & N/A \\
105 & 105 & N/A & N/A & N/A \\
119 & 140 & N/A & N/A & N/A \\
99 & 115 & 122 & 122 & 122 \\
100 & 100 & 95 & 97 & 100 \\
28 & N/A & N/A & N/A & N/A \\
92 & N/A & N/A & N/A & N/A \\
94 & 95 & 91 & 95 & 96 \\
100 & 100 & 97 & 100 & 100 \\
96 & 100 & 100 & 100 & 100 \\
121 & 101 & 56 & 63 & 88 \\
78 & 106 & 74 & 38 & 100 \\
12 & 73 & 6 & N/A & 21 \\
80 & 100 & 96 & 99 & 80 \\
84 & 100 & 96 & 99 & 83
\end{tabular}

\section{9}

90

/A

6

88

21

WEIGHTED AVERAGE PRICE (\$MMBTU) OF IMPORTS

\begin{tabular}{|c|c|c|c|c|}
\hline $\begin{array}{c}1998 \\
\text { qtr } \\
\# 4\end{array}$ & $\begin{array}{c}1999 \\
\text { qtr } \\
\mathbb{1 1}\end{array}$ & 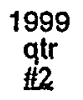 & $\begin{array}{c}1999 \\
\text { qtr } \\
\mathbb{\# 3}\end{array}$ & $\begin{array}{l}1999 \\
\text { qtr } \\
\# 4\end{array}$ \\
\hline 2.90 & 2.23 & 2.73 & 3.05 & 3.32 \\
\hline 2.13 & 2.11 & 2.17 & 2.39 & 2.54 \\
\hline 1.23 & 1.23 & 1.34 & 1.34 & 1.34 \\
\hline 3.93 & 4.16 & 4.16 & 4.16 & 4.16 \\
\hline 2.18 & 1.58 & 1.96 & 2.43 & 2.44 \\
\hline 2.18 & 1.88 & 2.34 & 2.80 & 2.91 \\
\hline 2.32 & 1.93 & 2.31 & 2.79 & 2.76 \\
\hline N/A & N/A & N/A & N/A & N/A \\
\hline 2.18 & 1.89 & 2.36 & 2.85 & 2.97 \\
\hline 2.09 & 1.89 & 2.36 & 2.85 & 3.01 \\
\hline 2.40 & 2.35 & 2.57 & 3.01 & 3.13 \\
\hline 1.81 & 1.76 & 1.81 & 1.89 & 1.97 \\
\hline 1.82 & 1.77 & 1.77 & 1.90 & 2.01 \\
\hline 2.25 & 2.19 & 2.28 & 2.28 & 2.35 \\
\hline N/A & N/A & N/A & N/A & N/A \\
\hline 2.16 & 2.37 & 2.52 & 3.04 & 3.18 \\
\hline 2.23 & 2.32 & 2.32 & 2.32 & 2.32 \\
\hline N/A & 1.91 & 2.29 & 2.64 & 2.58 \\
\hline N/A & N/A & N/A & N/A & 2.35 \\
\hline 2.47 & 2.76 & 2.41 & 2.48 & 2.81 \\
\hline 2.96 & 2.64 & N/A & N/A & N/A \\
\hline 3.14 & 2.79 & N/A & N/A & N/A \\
\hline 2.63 & 3.05 & N/A & N/A & N/A \\
\hline 2.49 & 2.24 & 2.48 & 2.72 & 3.20 \\
\hline 1.97 & 1.86 & 2.10 & 2.34 & 2.82 \\
\hline 2.41 & N/A & N/A & N/A & N/A \\
\hline 2.52 & N/A & N/A & N/A & N/A \\
\hline 2.15 & 1.88 & 2.29 & 2.73 & 2.71 \\
\hline 2.18 & 1.90 & 2.31 & 2.75 & 2.72 \\
\hline 2.32 & 1.93 & 2.31 & 2.79 & 2.79 \\
\hline 2.15 & 1.97 & 2.95 & 3.27 & 3.05 \\
\hline 4.66 & 2.40 & 2.95 & 4.31 & 3.42 \\
\hline 3.71 & 2.11 & 7.95 & N/A & 3.97 \\
\hline 2.25 & 1.89 & 2.30 & 2.74 & 2.71 \\
\hline 2.27 & 1.96 & 2.31 & 2.68 & 2.67 \\
\hline
\end{tabular}




\section{LONG-TERM IMPORTS}

\section{ACTUALIMPORTS AS APERCENT OF AUTHORIZED VOLUMES}

IMPORTER/EXPORTER

WISCONSIN FUEL \& LIGHT COMPANYIProgas Limited WISCONSIN FUEL \& LIGHT COMPANYTCGS LId.

WISCONSIN GAS COMPANY/ProGas Limited (I)

WISCONSIN GAS COMPANYIProGas Limited (II)

WISCONSIN GAS COMPANYITCGS Ltd.

WISCONSIN GAS COMPANYITCGS LId.

WISCONSIN POWER \& LIGHT COMPANYIProGas Limited

WISCONSIN POWER \& LIGHT COMPANYITCGS LId.

WISCONSIN PUBLIC SERVICE CORPORATION/ProGas Limited

WISCONSIN PUBLIC SERVICE CORPORATION/TCGS LId.

YANKEE GAS SERVICES CO./Alberta NE Gas (AEC Oil \& Gas Company)

YANKEE GAS SERVICES CO./Alberta NE Gas (TCGS Ltd. 1)

YANKEE GAS SERVICES CO./Alberta NE Gas (TCGS Litd. 2)

YANKEE GAS SERVICES CO./Alberta Northeast Gas (ProGas)

YANKEE GAS SERVICES CO./Alberta Northeast Gas (Producers Marketing)

\begin{tabular}{|c|c|c|c|c|}
\hline $\begin{array}{c}1998 \\
\text { qtr } \\
\# 4\end{array}$ & $\begin{array}{c}1999 \\
\text { qir } \\
\mathbb{\# 1}\end{array}$ & 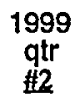 & $\begin{array}{c}1999 \\
\text { qtr } \\
\mathbb{1 3}\end{array}$ & $\begin{array}{c}1999 \\
\text { qtr } \\
\# 4\end{array}$ \\
\hline 96 & 103 & 100 & 99 & 100 \\
\hline 50 & 75 & 97 & 101 & 83 \\
\hline 83 & 100 & 99 & 77 & 76 \\
\hline 83 & 101 & 98 & 78 & 75 \\
\hline 87 & 100 & 98 & 77 & 65 \\
\hline 187 & 102 & 102 & 82 & 510 \\
\hline 99 & 99 & 96 & 99 & 99 \\
\hline 83 & 99 & 97 & 88 & 77 \\
\hline 79 & 84 & 96 & 84 & 100 \\
\hline 52 & 79 & 97 & 88 & 88 \\
\hline 101 & 101 & 101 & 101 & 101 \\
\hline 99 & 99 & 100 & 100 & 100 \\
\hline 101 & 99 & 100 & 100 & 100 \\
\hline 100 & 100 & 100 & 100 & 84 \\
\hline 100 & 100 & 100 & 100 & 100 \\
\hline
\end{tabular}

WEIGHTED AVERAGE PRICE (\$MMBTU) OFIMPORTS

$\begin{array}{ccccc}\begin{array}{c}1998 \\ \text { qtr }\end{array} & \begin{array}{c}1999 \\ \text { qtr } \\ \# 4\end{array} & \begin{array}{c}1999 \\ \text { qtr }\end{array} & \begin{array}{c}1999 \\ \text { qtr } \\ \# 3\end{array} & \begin{array}{c}1999 \\ \text { qtr } \\ \# 4\end{array} \\ 2.15 & 1.89 & 2.27 & 2.69 & 2.71 \\ 2.69 & 2.13 & 2.32 & 2.70 & 2.72 \\ 2.31 & 1.91 & 2.31 & 2.83 & 2.44 \\ 2.25 & 1.90 & 2.31 & 2.83 & 2.44 \\ 2.22 & 1.93 & 2.29 & 2.75 & 2.65 \\ 1.69 & 1.93 & 2.28 & 2.73 & 2.93 \\ 2.13 & 1.87 & 2.27 & 2.71 & 2.70 \\ 2.29 & 1.95 & 2.31 & 2.71 & 2.69 \\ 2.26 & 1.97 & 2.30 & 2.80 & 2.71 \\ 2.65 & 2.06 & 2.30 & 2.71 & 2.66 \\ 2.18 & 1.88 & 2.34 & 2.80 & 2.98 \\ 2.28 & 1.92 & 2.27 & 2.73 & 2.95 \\ 2.28 & 1.92 & 2.27 & 2.73 & 2.96 \\ 2.18 & 1.89 & 2.36 & 2.85 & 2.99 \\ 2.09 & 1.89 & 2.36 & 2.85 & 3.05\end{array}$


Quarterly Attachment B

Volumes and Weighted Average Prices

CANADA

LIQUEFIED NATURAL GAS

\begin{tabular}{|c|c|c|c|c|c|c|c|c|c|}
\hline $\begin{array}{l}\text { Year \& } \\
\text { Month }\end{array}$ & $\begin{array}{l}\text { Number of } \\
\text { Active } \\
\text { Contracts }\end{array}$ & $\begin{array}{c}\text { Volumes } \\
\text { (Mcf) }\end{array}$ & $\begin{array}{c}\text { Weighted } \\
\text { Avg. Price } \\
\text { (\$/MMBtu) }\end{array}$ & $\begin{array}{c}\text { Number of } \\
\text { Active } \\
\text { Contracts }\end{array}$ & $\begin{array}{l}\text { Algeria } \\
\text { Volumes } \\
\text { (Mcf) }\end{array}$ & $\begin{array}{r}\text { Weig } \\
\text { Everett, } \mathrm{MA}^{*}\end{array}$ & $\begin{array}{l}\text { ed Avg. Price } \\
\text { MMBtu) } \\
\text { Lake Charles, LA** }\end{array}$ & $\begin{array}{c}\text { Trinidad } \\
\text { Volumes } \\
\text { (Mcf) }\end{array}$ & $\begin{array}{c}\text { Weighted Avg. Price } \\
\text { (\$/MMBtu) } \\
\text { Everett, MA* }\end{array}$ \\
\hline \multicolumn{10}{|l|}{1997} \\
\hline January & 215 & $126,033,208$ & 3.48 & 2 & $7,301,466$ & 2.75 & 2.00 & 0 & 0.00 \\
\hline February & 212 & $109,281,936$ & 2.77 & 2 & $7,165,944$ & 3.01 & 2.00 & 0 & 0.00 \\
\hline March & 207 & $121,480,133$ & 1.93 & 1 & $2,530,039$ & 2.71 & 0.00 & 0 & 0.00 \\
\hline April & 207 & $114,763,739$ & 1.89 & 0 & 0 & 0.00 & 0.00 & 0 & 0.00 \\
\hline May & 206 & $119,618,080$ & 2.02 & 1 & $2,476,895$ & 0.00 & 2.00 & 0 & 0.00 \\
\hline June & 209 & $112,513,628$ & 2.12 & 2 & $4,906,283$ & 2.52 & 2.00 & 0 & 0.00 \\
\hline July & 204 & $111,325,034$ & 2.09 & 2 & $4,876,214$ & 2.50 & 2.00 & 0 & 0.00 \\
\hline August & 208 & $114,275,640$ & 2.09 & 2 & $7,456,909$ & 2.32 & 2.00 & 0 & 0.00 \\
\hline September & 208 & $107,958,822$ & 2.32 & 2 & $4,862,305$ & 2.39 & 2.00 & 0 & 0.00 \\
\hline October & 211 & $110,715,701$ & 2.67 & 2 & $4,963,689$ & 2.91 & 2.00 & 0 & 0.00 \\
\hline November & 214 & $116,855,355$ & 3.00 & 2 & $7,393,982$ & 2.95 & 2.00 & 0 & 0.00 \\
\hline December & 213 & $123,757,971$ & 2.40 & 2 & $7,492,080$ & 2.94 & 2.00 & 0 & 0.00 \\
\hline Total 1997 & & $1,388,579,246$ & 2.40 & & $61,425,806$ & 2.73 & 2.00 & 0 & 0.00 \\
\hline \multicolumn{10}{|l|}{1998} \\
\hline January & 211 & $119,517,456$ & 2.29 & 2 & $10,054,192$ & 2.84 & 2.00 & 0 & 0.00 \\
\hline February & 211 & $111,308,950$ & 2.13 & 3 & $7,220,277$ & 2.86 & 2.00 & 0 & 0.00 \\
\hline March & 210 & $120,179,623$ & 2.17 & 1 & $5,166,247$ & 2.84 & 0.00 & 0 & 0.00 \\
\hline April & 208 & $115,076,817$ & 2.23 & 1 & $2,398,524$ & 0.00 & 2.00 & 0 & 0.00 \\
\hline May & 207 & $111,680,329$ & 2.22 & 2 & $7,533,955$ & 2.44 & 2.00 & 0 & 0.00 \\
\hline June & 205 & $108,359,857$ & 2.07 & 2 & $4,961,946$ & 2.34 & 2.00 & 0 & 0.00 \\
\hline
\end{tabular}




\section{Quarterly Attachment B}

Volumes and Weighted Average Prices

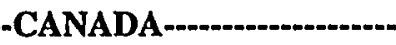

-...-.-

LIQUEFIED NATURAL GAS

\begin{tabular}{|c|c|c|c|c|c|c|c|c|c|}
\hline $\begin{array}{l}\text { Year \& } \\
\text { Month }\end{array}$ & $\begin{array}{c}\text { Number } \\
\text { of } \\
\text { Active } \\
\text { Contracts } \\
\end{array}$ & $\begin{array}{l}\text { Volumes } \\
\text { (Mcf) }\end{array}$ & $\begin{array}{l}\text { Weighted } \\
\text { Avg. Price } \\
\text { (\$/MMBtu) } \\
\end{array}$ & 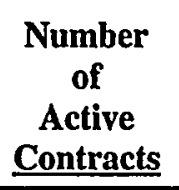 & $\begin{array}{c}\text { Algeria } \\
\text { Volumes } \\
\text { (Mcf) }\end{array}$ & \multicolumn{2}{|c|}{$\begin{array}{c}\text { Weighted Avg. Price } \\
\text { (\$/MMBtu) }\end{array}$} & $\begin{array}{c}\text { Trinidad } \\
\text { Volumes } \\
\text { (Mcf) }\end{array}$ & $\begin{array}{c}\text { Weighted Avg. Price } \\
\text { (\$/MMBtu) } \\
\text { Everett, MA* }\end{array}$ \\
\hline \multicolumn{10}{|l|}{1998} \\
\hline July & 193 & $110,819,028$ & 2.19 & 1 & $3,820,023$ & 0.00 & 2.00 & 0 & 0.00 \\
\hline August & 191 & $108,687,860$ & 1.97 & 1 & $2,205,882$ & 0.00 & 2.00 & 0 & 0.00 \\
\hline September & 192 & $107,592,471$ & 1.81 & 1 & $2,445,255$ & 0.00 & 1.57 & 0 & 0.00 \\
\hline October & 191 & $111,552,064$ & 2.02 & 1 & $5,216,505$ & 2.32 & 0.00 & 0 & 0.00 \\
\hline November & 202 & $115,882,342$ & 2.16 & 2 & $4,837,100$ & 2.33 & 2.00 & 0 & 0.00 \\
\hline December & 204 & $112,992,498$ & 2.23 & 2 & $7,500,950$ & 2.37 & 2.00 & 0 & 0.00 \\
\hline Total 1998 & & $1,353,649,295$ & 2.13 & & $63,360,856$ & 2.59 & 1.96 & 0 & 0.00 \\
\hline \multicolumn{10}{|l|}{1999} \\
\hline January & 205 & $122,344,648$ & 2.12 & 2 & $12,790,081$ & 2.29 & 2.00 & 0 & 0.00 \\
\hline February & 205 & $108,873,034$ & 2.02 & 2 & $7,211,930$ & 2.46 & 2.00 & 0 & 0.00 \\
\hline March & 207 & $117,960,699$ & 1.87 & 2 & $12,824,479$ & 2.32 & 2.00 & 0 & 0.00 \\
\hline April & 200 & $108,920,574$ & 1.92 & 2 & $7,180,718$ & 2.25 & 2.00 & 0 & 0.00 \\
\hline May & 197 & $111,168,762$ & 2.25 & 4 & $3,794,081$ & 1.91 & 2.00 & $4,031,987$ & 1.75 \\
\hline June & 201 & $107,532,391$ & 2.20 & 3 & $2,275,512$ & 0.00 & 2.00 & $6,619,872$ & 1.98 \\
\hline July & 195 & $114,308,602$ & 2.22 & 2 & $2,431,721$ & 0.00 & 2.00 & $6,598,926$ & 2.01 \\
\hline August & 196 & $112,930,969$ & 2.44 & 2 & $2,439,913$ & 0.00 & 2.00 & $5,512,175$ & 2.34 \\
\hline September & 198 & $106,880,296$ & 2.68 & 3 & $4,791,783$ & 2.36 & 2.00 . & $2,925,340$ & 2.55 \\
\hline October & 197 & $115,329,764$ & 2.52 & 3 & $4,975,657$ & 2.58 & 2.00 & $1,466,153$ & 2.79 \\
\hline November & 198 & $101,607,419$ & 2.89 & 2 & $2,440,328$ & 2.63 & 0.00 & $5,172,874$ & 2.74 \\
\hline December & 196 & $118,767,222$ & 2.33 & 2 & $2,492,066$ & 2.63 & 0.00 & $5,255,715$ & 2.70 \\
\hline Total 1999 & & $1,346,624,381$ & 2.28 & & $65,648,269$ & 2.36 & 2.00 & $37,583,042$ & 2.30 \\
\hline
\end{tabular}

* Landed Price

** Tailgate Price 


\section{VOLUME AND PRICE REPORT}

\section{Long-Term Imports}

Volumes (MMcf/d) \& Prices (\$/MMBTU) of Natural

Gas Imported During the Past 12 Months 01/01/99 - 12/31/99

Long-Term Importer

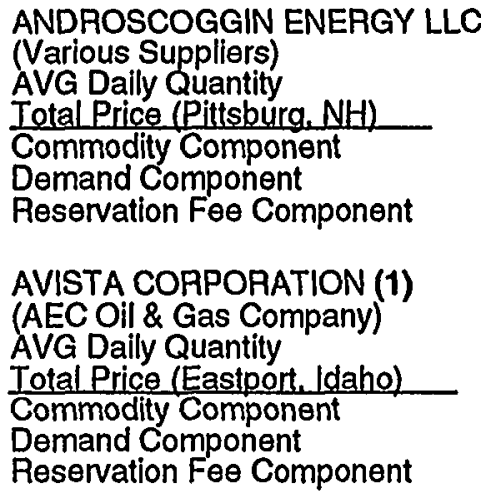

Reservation Fee Component

\begin{tabular}{|c|c|c|c|c|c|c|c|c|c|c|c|c|c|c|}
\hline $\begin{array}{l}\text { Auth. } \\
\text { Vols. }\end{array}$ & $\begin{array}{l}1999 \\
\text { Jan. }\end{array}$ & $\begin{array}{l}1999 \\
\text { Eab. }\end{array}$ & $\begin{array}{l}1999 \\
\text { Mar. }\end{array}$ & $\begin{array}{l}1999 \\
\text { Apr. }\end{array}$ & $\begin{array}{l}1999 \\
\text { May. }\end{array}$ & $\begin{array}{l}1999 \\
\text { Jun. }\end{array}$ & $\begin{array}{l}1999 \\
\text { Jul. }\end{array}$ & $\begin{array}{l}1999 \\
\text { Aug. }\end{array}$ & $\begin{array}{l}1999 \\
\text { Sep. }\end{array}$ & $\begin{array}{l}1999 \\
\text { Oct. }\end{array}$ & $\begin{array}{l}1999 \\
\text { Nov. }\end{array}$ & $\begin{array}{l}1999 \\
\text { Dec. }\end{array}$ & $\begin{array}{l}\text { 4Q999 } \\
\text { TOTAL MCf }\end{array}$ & $\begin{array}{l}\text { YTD } \\
\text { IOTALMCI }\end{array}$ \\
\hline 43.9 & $\begin{array}{r}0.0 \\
0.00 \\
0.00 \\
0.00 \\
0.00\end{array}$ & $\begin{array}{r}0.0 \\
0.00 \\
0.00 \\
0.00 \\
0.00\end{array}$ & $\begin{array}{r}0.0 \\
0.00 \\
0.00 \\
0.00 \\
0.00\end{array}$ & $\begin{array}{r}0.0 \\
0.00 \\
0.00 \\
0.00 \\
0.00\end{array}$ & $\begin{array}{r}0.0 \\
0.00 \\
0.00 \\
0.00 \\
0.00\end{array}$ & $\begin{array}{r}0.0 \\
0.00 \\
0.00 \\
0.00 \\
0.00\end{array}$ & $\begin{array}{r}0.0 \\
0.00 \\
0.00 \\
0.00 \\
0.00\end{array}$ & $\begin{array}{r}0.0 \\
0.00 \\
0.00 \\
0.00 \\
0.00\end{array}$ & $\begin{array}{r}0.0 \\
0.00 \\
0.00 \\
0.00 \\
0.00\end{array}$ & $\begin{array}{r}0.0 \\
0.00 \\
0.00 \\
0.00 \\
0.00\end{array}$ & $\begin{array}{r}9.0 \\
2.53 \\
1.76 \\
0.77 \\
0.00\end{array}$ & $\begin{array}{l}19.5 \\
2.72 \\
2.16 \\
0.56 \\
0.00\end{array}$ & 874,550 & 874,550 \\
\hline 25.6 & $\begin{array}{l}18.0 \\
2.05 \\
1.95 \\
0.10 \\
0.00\end{array}$ & $\begin{array}{l}18.4 \\
2.06 \\
1.95 \\
0.11 \\
0.00\end{array}$ & $\begin{array}{l}17.9 \\
2.05 \\
1.95 \\
0.10 \\
0.00\end{array}$ & $\begin{array}{r}0.0 \\
0.00 \\
0.00 \\
0.00 \\
0.00\end{array}$ & $\begin{array}{r}0.0 \\
0.00 \\
0.00 \\
0.00 \\
0.00\end{array}$ & $\begin{array}{r}0.0 \\
0.00 \\
0.00 \\
0.00 \\
0.00\end{array}$ & $\begin{array}{r}0.0 \\
0.00 \\
0.00 \\
0.00 \\
0.00\end{array}$ & $\begin{array}{r}0.0 \\
0.00 \\
0.00 \\
0.00 \\
0.00\end{array}$ & $\begin{array}{r}0.0 \\
0.00 \\
0.00 \\
0.00 \\
0.00\end{array}$ & $\begin{array}{r}0.0 \\
0.00 \\
0.00 \\
0.00 \\
0.00\end{array}$ & $\begin{array}{l}15.0 \\
2.55 \\
2.44 \\
0.11 \\
0.00\end{array}$ & $\begin{array}{l}22.6 \\
2.39 \\
2.29 \\
0.10 \\
0.00\end{array}$ & $1,148,852$ & $2,774,473$ \\
\hline 19.1 & $\begin{array}{l}15.2 \\
2.12 \\
1.58 \\
0.54 \\
0.00\end{array}$ & $\begin{array}{l}15.2 \\
1.81 \\
1.26 \\
0.55 \\
0.00\end{array}$ & $\begin{array}{l}15.3 \\
1.69 \\
1.15 \\
0.54 \\
0.00\end{array}$ & $\begin{array}{l}14.8 \\
1.71 \\
1.16 \\
0.55 \\
0.00\end{array}$ & $\begin{array}{l}15.3 \\
1.96 \\
1.42 \\
0.54 \\
0.00\end{array}$ & $\begin{array}{l}15.3 \\
1.98 \\
1.43 \\
0.55 \\
0.00\end{array}$ & $\begin{array}{l}15.3 \\
2.01 \\
1.47 \\
0.54 \\
0.00\end{array}$ & $\begin{array}{l}15.3 \\
2.10 \\
1.57 \\
0.53 \\
0.00\end{array}$ & $\begin{array}{l}15.0 \\
2.38 \\
1.82 \\
0.56 \\
0.00\end{array}$ & $\begin{array}{l}15.6 \\
2.26 \\
1.72 \\
0.54 \\
0.00\end{array}$ & $\begin{array}{l}15.3 \\
2.76 \\
2.23 \\
0.53 \\
0.00\end{array}$ & $\begin{array}{l}15.6 \\
2.12 \\
1.57 \\
0.55 \\
0.00\end{array}$ & $1,425,105$ & $5,571,761$ \\
\hline 16.8 & $\begin{array}{l}16.0 \\
1.73 \\
1.44 \\
0.29 \\
0.00\end{array}$ & $\begin{array}{l}17.3 \\
1.65 \\
1.32 \\
0.33 \\
0.00\end{array}$ & $\begin{array}{l}16.0 \\
1.59 \\
1.29 \\
0.30 \\
0.00\end{array}$ & $\begin{array}{l}16.0 \\
1.62 \\
1.31 \\
0.31 \\
0.00\end{array}$ & $\begin{array}{l}16.0 \\
1.91 \\
1.61 \\
0.30 \\
0.00\end{array}$ & $\begin{array}{l}16.0 \\
1.96 \\
1.65 \\
0.31 \\
0.00\end{array}$ & $\begin{array}{l}16.0 \\
2.05 \\
1.75 \\
0.30 \\
0.00\end{array}$ & $\begin{array}{l}16.0 \\
2.03 \\
1.73 \\
0.30 \\
0.00\end{array}$ & $\begin{array}{l}16.1 \\
2.39 \\
2.04 \\
0.35 \\
0.00\end{array}$ & $\begin{array}{l}16.1 \\
2.28 \\
1.98 \\
0.30 \\
0.00\end{array}$ & $\begin{array}{r}4.6 \\
2.72 \\
2.42 \\
0.30 \\
0.00\end{array}$ & $\begin{array}{l}17.2 \\
2.29 \\
1.98 \\
0.31 \\
0.00\end{array}$ & $1,171,666$ & $5,584,202$ \\
\hline 19.0 & $\begin{array}{l}15.0 \\
1.72 \\
1.72 \\
0.00 \\
0.00\end{array}$ & $\begin{array}{l}14.8 \\
1.64 \\
1.64 \\
0.00 \\
0.00\end{array}$ & $\begin{array}{l}14.8 \\
1.58 \\
1.58 \\
0.00 \\
0.00\end{array}$ & $\begin{array}{l}14.8 \\
1.09 \\
1.09 \\
0.00 \\
0.00\end{array}$ & $\begin{array}{l}14.8 \\
1.91 \\
1.91 \\
0.00 \\
0.00\end{array}$ & $\begin{array}{l}14.8 \\
1.95 \\
1.95 \\
0.00 \\
0.00\end{array}$ & $\begin{array}{l}14.8 \\
2.04 \\
2.04 \\
0.00 \\
0.00\end{array}$ & $\begin{array}{l}14.8 \\
2.01 \\
2.01 \\
0.00 \\
0.00\end{array}$ & $\begin{array}{l}14.8 \\
2.38 \\
2.38 \\
0.00 \\
0.00\end{array}$ & $\begin{array}{l}14.8 \\
2.33 \\
2.33 \\
0.00 \\
0.00\end{array}$ & $\begin{array}{l}15.8 \\
2.72 \\
2.72 \\
0.00 \\
0.00\end{array}$ & $\begin{array}{l}15.8 \\
2.27 \\
2.27 \\
0.00 \\
0.00\end{array}$ & $1,426,201$ & $5,474,630$ \\
\hline
\end{tabular}

Successor to Washington Water Power Company. . Successor to Washinglon Water Power Company. 


\section{VOLUME AND PRICE REPORT}

\section{Long-Term Imports}

Volumes (MMcf/d) \& Prices (\$MMBTU) of Natura

Gas Imported During the Past 12 Months 01/01/99 - 12/31/99

Long-Term Importer

\section{BAY STATE GAS COMPANY}

(Renaissance Energy Ltd.)

AVG Daily Quantity

Total Price (Niagara Falls, NY)

Commodity Component

Component

Reservation $\mathrm{Fe \theta}$ Component

\section{BEAR PAW ENERGY, L.L.C}

(Amoco Canada Resources Ltd.)

AVG Daily Quantity

Total Price (Portal. North Dakota)

Commodity Componen

Demand Component

Reservation Fee Component

BOSTON GAS COMPANY

(Alberta NE Gas (TCGS Ltd. 1))

AVG Daily Quantity

Total Price (Waddington. NY)

Commodity Component

Demand Component

Reservation $\mathrm{Fe \theta}$ Component

BOUNDARY GAS, INC. (1)

(TransCanada Pipelines Limited)

AVG Daily Quantity

Total Price (Niagara Falls. NY)

Commodity Component

Demand Component

Reservation Fee Component $\begin{array}{lll}1999 & 1999 \quad 1999\end{array}$

Vols.

6.4

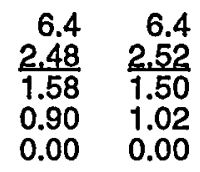

6.4
2.29
1.41
0.88
0.00

2.49
1.41
0.88

$\frac{2.44}{1.41}$

1.41
0.83

$0.00 \quad 0.00$

3.3

\begin{tabular}{rrr}
1.1 & 1.1 & 1.0 \\
1.16 & 1.17 & 1.24 \\
\hline 1.16 & 1.17 & 1.24 \\
0.00 & 0.00 & 0.00 \\
0.00 & 0.00 & 0.00
\end{tabular}

1.1
1.22
1.22
0.00
0.00

1.1
1.16
1.16

$\frac{1.16}{1.16}$

$\begin{array}{ll}0.00 & 0.00 \\ 0.00 & 0.00\end{array}$

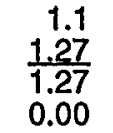

0.00
0.00
1.1
$\frac{1.19}{1.19}$
0.00

0.00
0.00

1.0
1.18
1.18
0.00
0.00

$\frac{1.0}{1.11}$

0.00
0.00

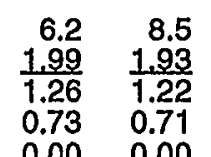

8.3
1.88
1.15
0.73
0.00

8.5
2.04
1.29
0.75
0.00

8.5
2.51
1.76
0.75

8.5
2.39
1.60
0.79
0.00

8.5
2.46
1.68
0.78

$\begin{array}{llll}0.00 & 0.00 & 0.00\end{array}$

8.5
2.76
1.98
0.78
0.00

8.5
$\frac{3.15}{2.36}$
0.79
0.00

8.5
2.80

$\frac{2.80}{2.01}$

0.79

$\frac{3.22}{2.43}$

2.43

0.79

2.36
1.57
0.79

0.79

83.0

$\begin{array}{llll}83.0 & 83.0 & 83.5 & 83.0 \\ 2.02 & 2.07 & \frac{1.93}{1.01} & \frac{1.89}{1.04} \\ 1.19 & 1.26 & 1.10 & 1.04 \\ 0.83 & 0.81 & 0.83 & 0.85 \\ 0.00 & 0.00 & 0.00 & 0.00\end{array}$

83.0
2.27
1.42
0.85
0.00

83.0
2.25
1.36
0.89
0.00

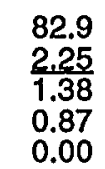

82.9
2.50
1.62
0.88

82.9
2.50
1.62
0.88
0.00

83.0
2.72
1.85
0.87
0.00

$\begin{array}{lll}83.0 & 77.9 & 83.0 \\ \frac{2.64}{1.77} & \frac{3.30}{2.43} & \frac{2.81}{1.95} \\ 0.87 & 0.87 & 0.86\end{array}$

$\begin{array}{lll}0.87 & 0.87 & 0.86\end{array}$

$\begin{array}{lll}0.87 & 0.87 & 0.86 \\ 0.00 & 0.00 & 0.00\end{array}$

BROOKLYN NAVY
(Crestar Energy)
AVG Daily Quantity

AVG Pally Quantity

Commodity Component

Demand Component

Reservation Fee Component

10.0

$\begin{array}{llll}10.9 & 10.8 & 10.8 & 10.9 \\ \frac{1.96}{1.39} & \frac{1.97}{1.32} & \frac{1.87}{1.27} & \frac{2.33}{1.41} \\ 0.57 & 0.65 & 0.60 & 0.92 \\ 0.00 & 0.00 & 0.00 & 0.00\end{array}$

10.9
2.76
1.87
0.89
0.00

10.8
2.71
1.75
0.96
0.00

10.7
$\frac{2.74}{1.81}$
0.93
0.00

10.8
$\frac{3.03}{2.10}$
0.93
0.00

10.8
$\frac{3.44}{2.48}$
0.96
0.00

10.9
3.08
2.15
0.93
0.00

$\begin{array}{r}10.9 \\ 3.53 \\ \hline 2.57\end{array}$

2.57

$0.96 \quad 0.93$

$0.00 \quad 0.00$

(1) As of July 1, 1998, Yankee Gas Services (9.5 MMc//day) is no longer importing under this authorization. 


\section{VOLUME AND PRICE REPORT}

\section{Long-Term Imports}

Volumes (MMct/d) \& Prices (\$MMBTU) of Natural Gas Imported During the Past 12 Months 01/01/99 - 12/31/99
Long-Term Importer

Auth

Vols.

BROOKLYN NAVY YARD COGEN. PARTNERS, L.P.

(PanCanadian Petroleum Ltd.)

AVG Daily Quantity

Total Price (Waddington. NY)

Commodity Component

Demand Component

Reservation Fee Component

BROOKLYN UNION GAS COMPANY

(Alberta NE Gas (AEC Oil \& Gas Company))

AVG Daily Quantity

Total Price (Waddington. NY)

Commodity Compone

Reservation Fee Component

\section{BROOKLYN UNION GAS COMPANY}

(Alberta NE Gas (TCGS Ltd. 1))

AVG Daily Quantity

Total Price (Waddington, NY)

Commodity Component

Demand Component

Reservation Fee Component

BROOKLYN UNION GAS COMPANY

(Alberta NE Gas (TCGS Ltd. 2))

AVG Daily Quantity

Total Price (Waddington. NY)

Commodity Component

Demand Component

Reservation Fee Component

BROOKLYN UNION GAS COMPANY

(Alberta Northeast Gas (ProGas))

AVG Daily Quantity

Total Price (Waddington, NY)

Commodity Component

Demand Component

Reservation Fee Component
15.0

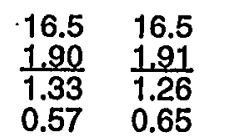

$\begin{array}{ll}0.00 & 0.00\end{array}$

4.0

\begin{tabular}{rr}
4.0 & 4.0 \\
1.90 & 1.95 \\
\hline 1.09 & 1.12 \\
0.81 & 0.83 \\
0.00 & 0.00
\end{tabular}

\begin{tabular}{r}
4.0 \\
1.80 \\
\hline 0.98 \\
0.82 \\
0.00
\end{tabular}

4.0

$\frac{2.03}{1.19}$

$0.00 \quad 0.00$

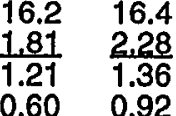

$0.60 \quad 0.92$

$0.00 \quad 0.00$
24.0

$\begin{array}{lll}24.0 & 24.0 & 24.0 \\ 1.99 & 1.93 & \frac{1.88}{1.15} \\ 1.26 & \frac{1.22}{1.26} & 0.73 \\ 0.73 & 0.71 & 0.00 \\ 0.00 & 0.00 & 0.00\end{array}$

18.0

\begin{tabular}{ll}
18.0 & 18.0 \\
1.99 & 1.93 \\
\hline 1.26 & 1.22 \\
0.73 & 0.71 \\
0.00 & 0.00
\end{tabular}

15.8

0.00

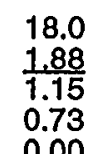

$\begin{array}{llll}1999 & 1999 & 1999 & 1999\end{array}$

Apr.

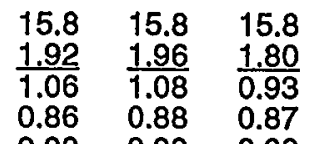

0.00
16.3
2.71
1.82 0.89 0.00

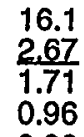

0.96
0.00

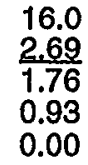

16.1
2.98
2.05
0.93

0.93
0.00
1999

4.0

$\frac{2.52}{1.67}$

1.67
0.85

0.00

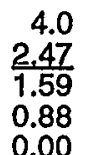

4.0
2.48
1.61
0.87
0.00

4.0
2.82
1.95
0.87
0.00

\begin{tabular}{r}
4.0 \\
3.11 \\
\hline 2.23 \\
0.88 \\
0.00
\end{tabular}

4.0
2.94
2.06

0.88
0.00

$\frac{3.34}{2.46}$

2.46

0.88

4.07
1.79

0.88

0.00

24.0
.29
.75
.00

24.0
2.51
1.76
0.75
0.00

24.0

24.0

24.0
2.76
1.98
0.78

24.0
3.15

$\frac{3.15}{2.36}$
0.79

0.00

24.0
2.80
2.01
0.79
0.00

$\begin{array}{r}24.0 \\ 3.22 \\ \hline\end{array}$

$\frac{3.22}{2.43}$

0.79

0.78

0.00

0.00

24.0

$\frac{2.36}{1.57}$

0.79

0.00

18.0
2.04

$\frac{2.04}{1.29}$

0.75

18.0
2.51
1.76
0.75
0.00

18.0
2.39
1.60
0.79
0.00

18.0
2.46
1.68
0.78
0.00

18.0
2.76
1.98
0.78
0.00

18.0
$\frac{3.15}{2.36}$
0.79
0.00

\begin{tabular}{l}
18.0 \\
2.80 \\
\hline 2.01 \\
0.79 \\
0.00
\end{tabular}

18.0
3.22
2.43
0.79
0.00 18.0
2.36
1.57
0.79
0.00

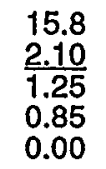

\begin{tabular}{l}
15.8 \\
2.53 \\
\hline 1.63 \\
0.90 \\
0.00
\end{tabular}

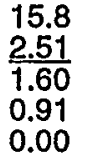

\begin{tabular}{l}
15.8 \\
2.51 \\
\hline 1.60 \\
0.91 \\
0.00
\end{tabular} \begin{tabular}{l}
15.8 \\
2.88 \\
\hline 1.97 \\
0.91 \\
0.00
\end{tabular}

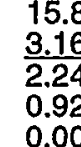

\begin{tabular}{lrr}
15.8 & 8.2 & 15.8 \\
2.99 & 3.38 & 2.79 \\
\hline 2.07 & 2.48 & 1.87 \\
0.92 & 0.90 & 0.92 \\
0.00 & 0.00 & 0.00
\end{tabular}
$1,656,000$

$6,570,000$

4099

TOTALMCf

YTD

$1,499,30$

$5,941,362$

363,768

$1,443,210$

$2,208,000$

$8,760,000$

$1,228,260$

$5,552,580$ 


\section{VOLUME AND PRICE REPORT}

\section{Long-Term Imports}

Volumes (MMcf/d) \& Prices (\$MMBTU) of Natural

Gas Imported During the Past 12 Months 01/01/99 - 12/31/99

Long-Term Importer

BROOKLYN UNION GAS COMPANY

(Alberta Northeast Gas (Producers Marketing)) AVG Daily Quantity

Total Price (Waddington. NY)

Commodity Componen

Demand Component

Reservation Fee Component

BROOKLYN UNION OF LONG ISLAND (1)

(Alberta NE Gas (AEC Oil \& Gas))

AVG Daily Quantity

Total Price (Waddington. NY)

Commodity Component

Demand Component

Reservation Fee Component

BROOKLYN UNION OF LONG ISLAND (2) (Alberta NE Gas (TCGS Ltd. 1))

AVG Daily Quantity

Total Price (Waddíngton. NY)

Commodity Component

Demand Component

Reservation Fee Component

BROOKLYN UNION OF LONG ISLAND (3)

(Alberta NE Gas (TCGS Ltd. 2))

AVG Daily Quantity

Total Price (Waddington. NY)

Commodity Component

Demand Component

Reservation Fee Component

BROOKLYN UNION OF LONG ISLAND (4)

(Alberta Northeast Gas (ProGas))

AVG Daily Quantity

Total Price (Waddington, NY)

Commodity Component

Demand Component

Reservation Fee Component

(1) Successor to Long Island Llghting Company.

(3) Succossor to Long Island Lighting Company.

(4) Successsor to Long Island Lighting Company.
Auth.

Auth

Auth. $1999 \quad 1999$

8.2

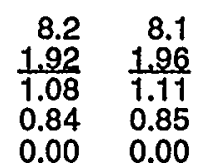

8.1
1.80
0.96
0.84
0.00
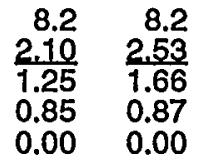

$\frac{2.45}{1.55}$

$\frac{2.51}{1.63}$

$0.90 \quad 0.88$

$0.00 \quad 0.00$

$\frac{2.88}{2.00}$

0.88

$0.00 \quad 0.00$

8.2
$\frac{3.16}{2.27}$
0.89
0.00

$\frac{2.29}{2.10}$

$\begin{array}{ll}0.89 & 0.93 \\ 0.00 & 0.00\end{array}$

2.79

1.90

0.89
0.00

3.1

$\begin{array}{rrr}3.1 & 3.1 & 3.1 \\ 1.90 & \frac{1.95}{1.12} & 1.80 \\ 1.09 & 0.98 \\ 0.81 & 0.83 & 0.82 \\ 0.00 & 0.00 & 0.00\end{array}$

3.1
2.03
1.19
0.84
0.00

3.1
$\frac{2.52}{1.67}$
0.85
0.00

3.1
2.47
1.59
0.88
0.00
3.1
2.48

$\frac{2.48}{1.61}$

3.1
2.82
1.95
0.87

3.1
$\frac{3.11}{2.23}$

$\begin{array}{r}3.1 \\ 2.67 \\ \hline 1.79\end{array}$

$\frac{2.67}{1.79}$

0.88

232,940

$1,069,685$

30.4

\begin{tabular}{lll}
30.4 & 30.4 & 30.4 \\
1.99 & 1.93 & 1.88 \\
\hline 1.26 & $\frac{1.22}{1.15}$ \\
0.73 & 0.71 & 0.73
\end{tabular}

\section{4}

$\frac{2.04}{129}$

30.4
$\frac{2.51}{1.76}$

$\frac{2.51}{1.76}$
0.75

30.4
2,39
1.60
0.79

0.00

$0.00 \quad 0.00$

30.4
2.46
1.68
0.78
0.00

30.4
2.76
1.98
0.78

\begin{tabular}{l}
30.4 \\
3.15 \\
\hline
\end{tabular}

$\frac{3.15}{2.36}$

0.79

0.00

$30.4 \quad 19.3$

$\frac{2.80}{2.01}$

2.01

0.79
0.00

$\frac{3.22}{2.43}$

2.43

0.79
0.00

30.4

$\frac{2.36}{1.57}$

1.57
0.79
0.00

13.5

$\begin{array}{llll}13.5 & 13.5 & 13.5 & 13.5 \\ 1.99 & 1.93 & 1.88 & 2.04 \\ 1.26 & .1 .22 & 1.15 & 1.29 \\ 0.73 & 0.71 & 0.73 & 0.75 \\ 0.00 & 0.00 & 0.00 & 0.00\end{array}$

13.5
2.51
1.76
0.75
0.00

$\begin{array}{ll}13.5 & 13.5 \\ 2.39 & 2.46 \\ 1.60 & 1.68 \\ 0.79 & 0.78 \\ 0.00 & 0.00\end{array}$

13.5
2.76
1.98
0.78
0.00

13.5
3.15
2.36
0.79
0.00

13.5

13.5
2.80
2.01

2.01
0.79

8.6
3.22

$\frac{3.22}{2.43}$

13.5

$\frac{2.36}{1.57}$

1.57
0.79

$\begin{array}{lll}0.00 & 0.00 & 0.00\end{array}$

11.9

\begin{tabular}{ll}
11.9 & 11.9 \\
1.92 & 1.96 \\
\hline 1.06 & 1.08 \\
0.86 & 0.88 \\
0.00 & 0.00
\end{tabular}

11.9
1.80
0.93
0.87
0.00

11.9
2.10
1.25
0.85
0.00

11.9
2.53
1.63
0.90
0.00

11.9
2.45
1.52
0.93
0.00

\begin{tabular}{lr}
11.9 & 11.9 \\
2.51 & 2.88 \\
\hline 1.60 & 1.97 \\
0.91 & .0 .91 \\
0.00 & 0.00
\end{tabular}

11.9
$\frac{3.16}{2.24}$
0.92
0.00

11.9
2.99
2.07
0.92
0.00

3.7
3.38
2.48
0.90
0.00

11.9

$\frac{2.79}{1.87}$

0.92

0.00

\section{$2,462,400 \quad 10,761,600$}

$1,093,500$

$4,779,000$

846,821

$4,090,061$ 


\section{VOLUME AND PRICE REPORT}

\section{Long-Term Imports}

Volumes (MMcf/d) \& Prices (\$MMBTU) of Natura Gas Imported During the Past 12 Months 01/01/99 - 12/31/99

Long-Term Importer

BROOKLYN UNION OF LONG ISLAND (1)

(Alberta Northeast Gas (Producers Marketing))

AVG Daily Quantity

total Price (Waddington, NY)

Commodity Component

Demand Component

Reservation Fee Component

\section{CASCADE NATURAL GAS. COPORATION (2)}

(IGI Resources, Inc.)
AVG Daily Quantity

Total Price (Eastport, Idaho)

Commodity Component

Demand Component

Reservation Fee Component

CASCADE NATURAL GAS CORPORATION

(Duke Energy Trad. \& Mktg. Svcs., LLC)

AVG Daily Quantity

Total Price (Sumas, Washington)

Commodity Component

Demand Component

Reservation $\mathrm{Fe \theta}$ Component

\section{CASCADE NATURAL GAS CORPORATION}

(Engage Energy Canada)

AVG Daily Quantity

Total Price (Eastport, Idaho)

Commodity Componen

Demand Component

Reservation Fee Component

CASCADE NATURAL GAS CORPORATION (3)

(Engage Energy Canada)

AVG Daily Quantity

Total Price (Sumas, Washington)

Commodity Component

Reservation Fee Component
Auth

6.2

\begin{tabular}{r}
6.2 \\
1.92 \\
\hline 1.08 \\
0.84 \\
0.00
\end{tabular}

7.4

\begin{tabular}{rrr}
7.3 & 7.8 & 7.3 \\
2.10 & 2.08 & 2.11 \\
\hline 1.80 & 1.79 & 1.80 \\
0.22 & 0.20 & 0.23 \\
0.08 & 0.09 & 0.08
\end{tabular}

12.0

\begin{tabular}{rrrr}
11.6 & 9.1 & 11.7 & 11.8 \\
3.11 & $\frac{1.88}{2.18}$ & 2.18 & 1.60 \\
\hline 2.37 & 1.21 & 1.54 & 0.98 \\
0.52 & 0.56 & 0.50 & 0.53
\end{tabular}

0.22

0.11

0.14

33.2

$\begin{array}{lll}23.5 & 24.7 & 25.6 \\ 2.30 & 2.17 & 2.03 \\ 1.89 & 1.74 & 1.66 \\ 0.29 & 0.31 & 0.26 \\ 0.12 & 0.12 & 0.11\end{array}$

30.0

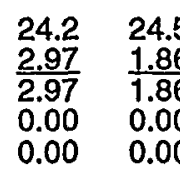

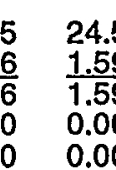

\begin{tabular}{rr}
0.0 & 0.0 \\
0.00 & 0.00 \\
\hline 0.00 & 0.00 \\
0.00 & 0.00
\end{tabular}

$\begin{array}{ll}0.00 & 0.00 \\ 0.00 & 0.00\end{array}$ $\begin{array}{llllll}1999 & 1999 & 1999 & 1999 & 1999 & 1999\end{array}$

1999

Jun. 
VOLUME AND PRICE REPORT

\section{Long-Term Imports}

Volumes (MMcf/d) \& Prices (\$MMBTU) of Natural

Gas Imported During the Past 12 Months 01/01/99 - 12/31/99
Long-Term Importer

\section{CENTRAL HUDSON COMPANY}

(Alberta NE Gas (AEC Oil \& Gas Company))

AVG Daily Quantity

Total Price (Waddington. NY)

Commodity Component

Demand Component

Reservation $\mathrm{Fee}$ Component

CENTRAL HUDSON COMPANY

(Alberta NE Gas (TCGS Ltd. 1))

AVG Daily Quantity

Total Price (Waddington. NY)

Commodity Component

Demand Component

Reservation Fee Component

\section{CENTRAL HUDSON COMPANY}

(Alberta NE Gas (TCGS Ltd. 2))

AVG Daily Quantity

Total Price (Waddington. NY)

Commodity Component

Demand Component

Reservation Fee Component

\section{CENTRAL HUDSON COMPANY}

(Alberta Northeast Gas (ProGas))

AVG Daily Quantity

Total Price (Waddington, NY)

Commodity Component

Demand Component

Reservation Fee Component

\section{CENTRAL HUDSON COMPANY}

(Alberta Northeast Gas (Producers Marketing))

AVG Daily Quantity

Total Price (Waddington. NY)

Commodity Component

Demand Componen

Reservation Fee Componen
Auth.

Ao

0.1
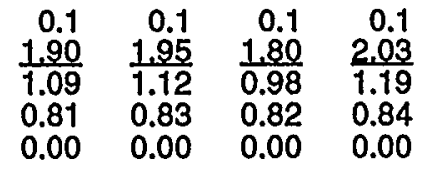

0.1
2.52
1.57

0.00

14.1

14.1
1.99
1.26
0.73
0.00

14.1
1.93
1.22
0.71
0.00

14.1

$\frac{1.88}{1.15}$

14.1
2.04
1.29
0.75
0.00

14.1
$\frac{2.51}{1.76}$
0.75

14.1
2.39

0.75
0.00

1.60
0.79

0.00
1.5

$\begin{array}{rrrr}1.5 & 1.5 & 1.5 & 1.5 \\ \frac{1.99}{1.26} & \frac{1.93}{1.22} & \frac{1.88}{1.15} & \frac{2.04}{1.29} \\ 0.73 & 0.71 & 0.73 & 0.75\end{array}$

0.00

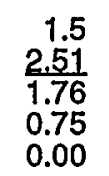

1.5
2.39
1.60
0.79
0.00

1.5
2.46
1.68
0.78
0.00

1.5
$\frac{2.76}{1.98}$

1.98

0.00
1.5
$\frac{3.15}{2.36}$
0.79
0.00

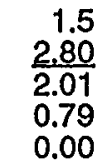

1.3

\begin{tabular}{rrrr}
1.3 & 1.3 & 1.3 & 1.3 \\
1.92 & 1.96 & 1.80 & 2.10 \\
\hline 1.06 & 1.08 & 0.93 & 1.25 \\
0.86 & 0.88 & 0.87 & 0.85 \\
0.00 & 0.00 & 0.00 & 0.00
\end{tabular}

1.3
$\frac{2.53}{1.63}$
0.90
0.00

1.3
2.45
1.52
0.93
0.00

$\frac{1.3}{1.51}$

$\frac{2.51}{1.60}$

0.91

0.00

1.3
2.88
1.97

0.91

0.91
0.00

1.3
$\frac{3.16}{2.24}$
0.92

0.00

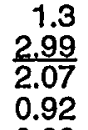

$\frac{2.99}{2.07}$

0.92
0.00

$\frac{3.38}{2.48}$

2.48

0.00

$\frac{2.79}{1.87}$

0.92

0.00

3.0

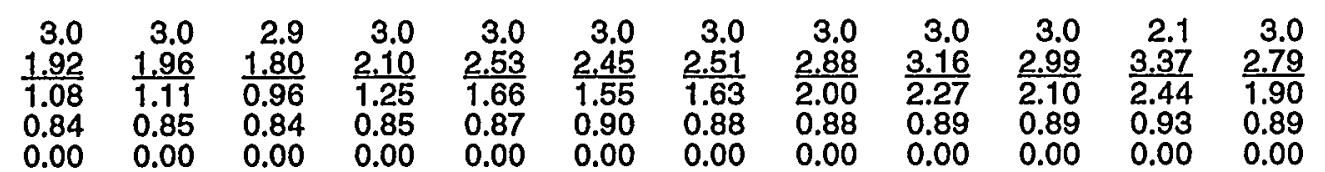

4099

TOTAL MCF

7,296

0.1
2.67
1.79

0.88

0.00

$1,142,100$

$.4,991,400$

$\begin{array}{ll}8.9 & 14.1 \\ \frac{3.22}{2.43} & \frac{2.36}{1.57} \\ 0.79 & 0.79 \\ 0.00 & 0.00\end{array}$

$\begin{array}{r}1.0 \\ 3.22 \\ \hline 2.43\end{array}$

0.79

1.5
2.36
1.57

0.79

0.00

121,531

531,274

144,844

505,204

248,012

$1,059,910$ 
VOLUME AND PRICE REPORT

\section{Long-Term Imports}

Volumes (MMct/d) \& Prices (\$/MMBTU) of Natural

Gas Imported During the Past 12 Months 01/01/99 - 12/31/99
Leng-Term Importer

CHEVRON NATURAL GAS SERVICES, INC. (1) (Chevron Canada Resources)

AVG Daily Quantity

Total Price (Eastport, Idaho)

Commodity Component

Demand Component

Reservation Fee Component

CHEVRON U.S.A. INC. (2)

(Chevron Canada Resources)

AVG Daily Quantity

Total Price (Eastport. Jdaho)

Commodity Component

Demand Component

Reservation Fee Component

CHEVRON U.S.A. INC. (3)

(Chevron Canada Resources)

AVG Daily Quantity

Total Price (Champlain. NY)

Commodity Component

Demand Component

Reservation Fee Component

CITY OF BURBANK, CALIFORNIA (4)

(Engage Energy Canada, L.P.)

AVG Daily Quantity

Total Price (Eastport, Idaho)

Commodity Component

Demand Component

Reservation Fee Component

CITY OF DULUTH, MINNESOTA

(ProGas Limited)

AVG Daily Quantity

Total Price (Noyes. Minnesota)

Commodity Component

Demand Component

Reservation Fee Component

\section{A}

ols. Jan. Feb Mar. Apr Mav Jun. Jul. Aug Sep oct. Nov Dec.

\begin{tabular}{|c|c|}
\hline $\begin{array}{l}19.0 \\
2.44 \\
2.11 \\
0.33 \\
0.00\end{array}$ & $\begin{array}{l}18.8 \\
2.47 \\
2.11 \\
0.36 \\
0.00\end{array}$ \\
\hline
\end{tabular}

74.0

$\begin{array}{lll}61.3 & 63.1 & 35 \\ 1.81 & 1.41 & 1 \\ \frac{1.81}{1.41} & \frac{1}{1} \\ 0.00 & 0.00 & 0.00 \\ 0.00 & 0.00 & 0.00\end{array}$

1.5

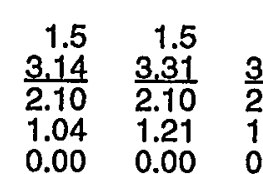

$\begin{array}{rr}1.5 & 1.6 \\ \frac{3.17}{2.10} & \frac{3.23}{2.10} \\ 1.07 & 1.13 \\ 0.00 & 0.00\end{array}$

$\begin{array}{rrr}1.6 & 1.5 & 1.5 \\ \frac{3.21}{2.10} & \frac{3.28}{2.10} & \frac{3.19}{2.10} \\ 1.11 & 1.18 & 1.09 \\ 0.00 & 0.00 & 0.00\end{array}$

1.5
3.19

$\begin{array}{lll}1.00 & 0.00 & 0.00\end{array}$

$16.4 \quad 15.0$

2.11

0.38
0.00

$\frac{2.51}{2.11}$

0.40

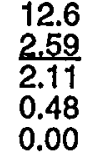

16.1

$\frac{2.48}{2.11}$

2.11
0.37

0.37
0.00

18.0
2.45
2.11
0.34
0.00

18.0

$\frac{2.46}{2.11}$

18.7

$\frac{2,45}{2.11}$

18.3

$\frac{2.59}{2.23}$

16.7

$\frac{2.61}{2.23}$

0.38

0.38
0.00

4.8

\begin{tabular}{rrrr}
4.0 & 4.7 & 4.7 & 4.2 \\
2.02 & 2.02 & 2.02 & 2.02 \\
\hline 1.74 & 1.74 & 1.74 & 1.74 \\
0.28 & 0.28 & 0.28 & 0.28
\end{tabular}

$\begin{array}{ll}0.28 & 0.28 \\ 0.00 & 0.00\end{array}$

0.28
0.00

6.1

\begin{tabular}{rrr}
0.0 & 0.0 & 0.0 \\
0.00 & 0.00 & 0.00 \\
\hline 0.00 & 0.00 & 0.00 \\
0.00 & 0.00 & 0.00 \\
0.00 & 0.00 & 0.00
\end{tabular}

0.00

61.1
$\frac{1.43}{1.43}$
0.00

$\begin{array}{lll}54.5 & 60.6 & 46.9\end{array}$

$\frac{1.77}{1.77} \quad \frac{1.78}{1.78}$

$0.00 \quad 0.00$

$\frac{1.80}{1.80}$

1.80
0.00
0.00

46.8

0.00

0.00

0.00 \begin{tabular}{llll}
59.0 & 60.3 & 61.0 & 61.4 \\
$\frac{2.23}{2.23}$ & $\frac{2.15}{2.15}$ & $\frac{2.61}{2.61}$ & $\frac{2.03}{2.03}$ \\
\hline & 0.00 & 0.00 & 0.00
\end{tabular}

$\begin{array}{llll}0.00 & 0.00 & 0.00 & 0.00\end{array}$

$\begin{array}{lllll}0.00 & 0.00 & 0.00 & 0.00 & 0.00\end{array}$

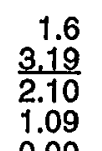

1.6
$\frac{3.31}{2.10}$
1.21

1.4
$\frac{3.24}{2.10}$
1.14

$\frac{3.45}{2.37}$

1.18

1.4
$\frac{3.53}{2.37}$
1.16 1.16
0.00

$\begin{array}{rr}4.6 & 4.9 \\ 2.02 & 2.02 \\ 1.74 & 1.74 \\ 0.28 & 0.28\end{array}$

3.8
$\frac{1.95}{1.64}$
0.31
0.00

4.8
$\frac{1.90}{1.64}$
0.26

4.3
$\frac{1.94}{1.64}$
0.30

$\frac{4.8}{1.61}$

0.0

$\frac{0.00}{0.00}$

0.00

$\begin{array}{lll}0.27 & 0.00 & 0.00 \\ 0.00 & 0.00 & 0.00\end{array}$

\section{$5,602,754$}

132,521

555,235

(1) Supplies Hermiston cogeneration facility.

(2) Supplies are resold to Dynegy Marketing \& Trade, primarily for markels in California.

(3) Gas delivered to Georgia-Pacific's Plattsburg. N.Y. plant. 


\section{VOLUME AND PRICE REPORT}

\section{Long-Term Imports}

Volumes (MMct/d) \& Prices (\$MMBTU) of Natural

Gas Imported During the Past 12 Months 01/01/99 - 12/31/99

Long-Term Importer

CITY OF GLENDALE, CALIFORNIA (Engage Energy Canada, L.P.) AVG Daily Quantity

Total Price (Eastport, Idaho)

Commodity Componen

Demand Component

Reservation Fee Component

CITY OF PASADENA, CALIFORNIA

(Engage Energy Canada)

AVG Daily Quantity

Total Price (Eastport. Idaho)

Commodity Component

Demand Component

Reservation Fee Component

\section{COENERGY TRADING COMPANY}

(Various Suppliers)

AVG Daily Quantity

Total Price (Pittsburg. NH)

Commodity Componen

Demand Component

Reservation Fee Component

\section{COLONIAL NATURAL GAS}

(Alberta NE Gas (TCGS Ltd. 1))

AVG Daily Quantity

Total Price (Waddington. NY)

Commodity Componen

Demand Component

Reservation Fee Component

COMMONWEALTH GAS COMPANY

(Alberta NE Gas (TCGS Ltd. 1))

AVG Daily Quantity

Total Price (Waddington, NY)

Commodity Component

Demand Component

Reservation Fee Component
Auth.

1999

1999

1999
Mar.

1999

Apr.

Mav

Jun.

Jul

4.1

4.2
2.00
1.74
0.26
0.00

4.1
2.04
1.74
0.30
0.00

3.8
2.04
1.74
0.30

$\begin{array}{r}3.9 \\ 2.02 \\ \hline\end{array}$

$\frac{2.02}{1.74}$

3.7
2.03
1.74

$\frac{2.03}{1.74}$

4.0
2.02
1.74

2.02
1.74
0.28

0.28
0.00

$\begin{array}{r}4.1 \\ 1.91 \\ \hline\end{array}$

$\frac{1.91}{1.64}$

0.27
0.00

$\begin{array}{lll}0.00 & 0.00 & 0.00\end{array}$

4.1
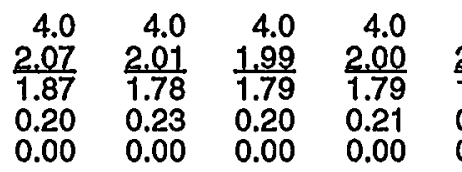

3.8
2.01
1.79

$\frac{4.00}{1.78}$

$0.00 \quad 0.00$

30.0

$\begin{array}{rrr}0.0 & 0.0 & 8.8 \\ \frac{0.00}{0.00} & \frac{0.00}{0.00} & \frac{1.98}{1.98} \\ 0.00 & 0.00 & 0.00\end{array}$

$\begin{array}{lll}0.00 & 0.00 & 0.00 \\ 0.00 & 0.00 & 0.00\end{array}$

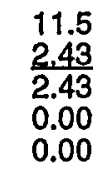

16.8
2.38
2.38
0.00
0.00

9.1
2.42
2.42
0.00
0.00

36.4
2.45
2.45
0.00
0.00

6.0

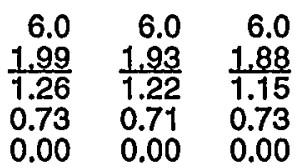

6.0
2.04
1.29
0.75
0.00

6.0
2.51
1.76
0.75
0.00

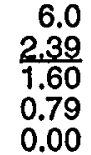

6.0
2.46
1.68
0.78

0.00

4.5

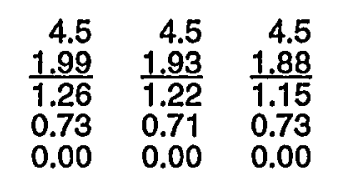

\begin{tabular}{r}
4.5 \\
2.04 \\
\hline 1.29 \\
0.75 \\
0.00
\end{tabular}

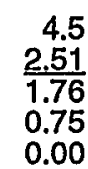

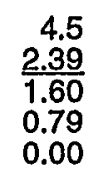

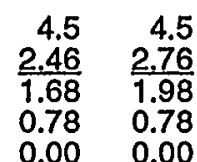

$\frac{1.91}{1.64}$

1.64
0.27

0.27
0.00

4.02
1.64

1.64
4.5
3.15
2.36
0.79
0.00
4Q99

TOTAL MC

382,194

$1,471,330$

$\frac{1.64}{1.64}$

$\frac{1.64}{1.64} \quad \frac{1.64}{1.64}$

$\begin{array}{lll}0.00 & 0.00 & 0.00\end{array}$

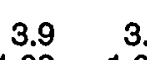

\begin{tabular}{rr}
3.9 & 4.0 \\
1.88 & 1.90 \\
\hline 1.67 & 1.68 \\
0.21 & 0.22 \\
0.00 & 0.00
\end{tabular}

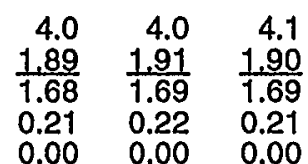

371,481

$1,457,851$

4.4
286

$\frac{2.86}{2.86}$

2.86
0.00

0.00

33.9
2.98
2.98
0.00
0.00

29.4

2.93

41.4
$\frac{3.33}{3.33}$

60.8
2.27

$\frac{2.27}{2.27}$

0.00

0.00

0.00
0.00

$4,034,909$

$7,725,159$

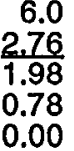

6.0
3.15
2.36
0.79

6.0
2.80
2.01
0.79

3.8

6.0

$\frac{3.22}{2.43}$

0.79

$\frac{2.36}{1.57}$

1.57
0.79

0.00

486,000

$2,124,000$

$\begin{array}{rrr}4.5 & 2.9 & 4.5 \\ \frac{2.80}{2.01} & \frac{3.22}{2.43} & \frac{2.36}{1.57} \\ 0.79 & 0.79 & 0.79 \\ 0.00 & 0.00 & 0.00\end{array}$

364,500

$1,593,000$ 


\section{VOLUME AND PRICE REPORT}

\section{Long-Term Imports}

Volumes (MMcf/d) \& Prices (\$MMBTU) of Natural

Gas Imported During the Past 12 Months 01/01/99 - 12/31/99

Long-Term Importer

CONNECTICUT NATURAL GAS

(Alberta NE Gas (AEC Oil \& Gas Company)) AVG Daily Quantity

Total Price (Waddington. NY)

Commodity Component

Demand Component

Reservation Fee Component

CONNECTICUT NATURAL GAS

(Alberta NE Gas (TCGS Ltd. 1))

AVG Daily Quantity

Total Price (Waddington. NY)

Commodity Component

Demand Component

Reservation Fee Component

CONNECTICUT NATURAL GAS

(Alberta NE Gas (TCGS Ltd. 2))

AVG Daily Quantity

Total Price (Waddington, NY)

Commodity Component

Demand Component

Reservation Fee Component

CONNECTICUT NATURAL GAS

(Alberta Northeast Gas (ProGas))

AVG Daily Quantity

Total Price (Waddington. NY)

Commodity Component

Demand Component

Reservation Fee Component

CONNECTICUT NATUAAL GAS

(Alberta Northeast Gas (Producers Marketing)) AVG Daily Quantity

Total Price (Waddington, NY)

Demand Component

Reservation Fee Component
Auth

1.2

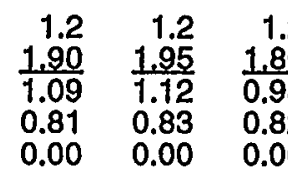

11.5

\begin{tabular}{ll}
11.5 & 11.5 \\
1.99 & 1.93 \\
\hline 1.26 & 1.22 \\
0.73 & 0.71 \\
0.00 & 0.00
\end{tabular}

\begin{tabular}{l}
11.5 \\
1.88 \\
\hline 1.15 \\
0.73 \\
0.00
\end{tabular}

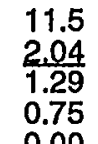

11.5
2.51
1.76
0.75
0.00

11.5
2.39

0.00

5.3

\begin{tabular}{rrrr}
5.3 & 5.3 & 5.3 & 5.3 \\
1.99 & $\frac{1.93}{1.22}$ & $\frac{1.88}{1.15}$ & 2.04 \\
\hline 1.26 & 1.29 \\
0.73 & 0.71 & 0.73 & 0.75 \\
0.00 & 0.00 & 0.00 & 0.00
\end{tabular}

5.3
2.51
1.76
0.75
0.00

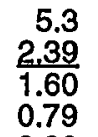

5.3
2.46
1.68
0.78
0.00

4.6

\begin{tabular}{rr}
4.6 & 4.6 \\
1.92 & 1.96 \\
\hline 1.06 & 1.08 \\
0.86 & 0.88 \\
0.00 & 0.00
\end{tabular}

4.6

$4.6 \quad 4.6$

$\frac{1.80}{0.93}$

$0.93 \quad 1.25$

$0.00 \quad 0.00$

0.00

2.4

\begin{tabular}{r}
2.4 \\
1.92 \\
\hline 1.08 \\
0.84 \\
0.00
\end{tabular}

\begin{tabular}{r}
2.4 \\
1.96 \\
\hline 1.11 \\
0.85 \\
0.00
\end{tabular}

\begin{tabular}{r}
2.4 \\
1.80 \\
\hline 0.96 \\
0.84 \\
0.00
\end{tabular}

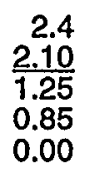

2.4
2.53
1.66
0.87
0.00

2.4
2.45
1.55
0.90
0.00

\begin{tabular}{r}
2.4 \\
2.51 \\
\hline 1.63 \\
0.88 \\
0.00
\end{tabular}

\begin{tabular}{r}
2.4 \\
2.88 \\
\hline 2.00 \\
0.88 \\
0.00
\end{tabular}

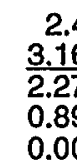

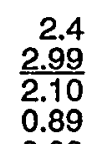

$\frac{2.99}{2.10}$

0.89

0.00
$4 Q 99$

IQTAL Mc

97,686

$\begin{array}{r}0.8 \\ 3.34 \\ \hline\end{array}$

3.34
0.86

$\begin{array}{ll}0.88 & 0.88\end{array}$

\begin{tabular}{r}
1.2 \\
2.67 \\
\hline 1.79 \\
0.88 \\
0.00
\end{tabular}

97,686

.

11.5
2.80

$\frac{2.80}{2.01}$

0.79

\begin{tabular}{rr}
7.3 & 11.5 \\
3.22 & 2.36 \\
\hline 2.43 & 1.57 \\
0.79 & 0.79 \\
0.00 & 0.00
\end{tabular}

931,500

$4,071,000$

.

425,250

$1,858,500$

\begin{tabular}{lrr}
2.80 & 3.22 & 2.36 \\
\hline
\end{tabular}

$2.01 \quad 2.43 \quad 1.57$

$\begin{array}{lll}0.79 & 0.79 & 0.79 \\ 0.00 & 0.00 & 0.00\end{array}$

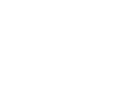

332,496

$1,593,756$

\begin{tabular}{rr}
1.5 & 4.6 \\
3.38 & 2.79 \\
\hline 2.48 & 1.87 \\
0.90 & 0.92 \\
0.00 & 0.00
\end{tabular}

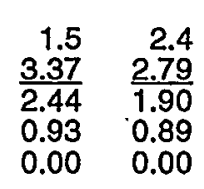




\section{VOLUME AND PRICE REPORT}

\section{Long-Term Imports}

Volumes (MMcf/d) \& Prices (\$MMBTU) of Natural

Gas Imported During the Past 12 Months 01/01/99 - 12/31/99

Long-Term importer

CONSOLIDATED EDISON COMPANY OF N.Y.

(Alberta NE Gas (AEC Oil \& Gas))

AVG Daily Quantity

Total Price (Waddington, NY)

Commodity Component

Demand Component

Reservation Fee Component

CONSOLIDATED EDISON COMPANY OF N.Y.

(Alberta NE Gas (ProGas))

AVG Daily Quantity

Total Price (Waddington, NY)

Commodity Component

Demand Component

Reservation Fee Component

CONSOLIDATED EDISON COMPANY OF N.Y.

(Alberta NE Gas (Producers Marketing))

AVG Daily Quantity

Total Price (Waddington. NY)

Commodity Componen

Demand Component

Reservation Fee Component

CONSOLIDATED EDISON COMPANY OF N.Y.

(Alberta NE Gas (TCGS Ltd. 1))

AVG Daily Quantity

Total Price (Waddington. NY)

Commodity Component

Demand Component

Reservation Fee Component

CONSOLIDATED EDISON COMPANY OF N.Y.

(Alberta NE Gas (TCGS Lid. 2))

AVG Daily Quantity

Total Price (Waddington, NY)

Commodity Component

Demand Component

Reservation Fee Component
Auth

1.1

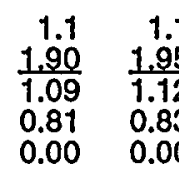

5.3

\begin{tabular}{rr}
5.3 & 5.3 \\
1.92 & 1.96 \\
\hline 1.06 & 1.08 \\
0.86 & 0.88 \\
0.00 & 0.00
\end{tabular}

5.3
1.80
0.93
0.87

$\begin{array}{ll}0.00 & 0.00\end{array}$

2.6

\begin{tabular}{rrr}
2.6 & 2.6 & 2.6 \\
1.92 & 1.96 & 1.80 \\
\hline 1.08 & 1.11 & 0.96 \\
0.84 & 0.85 & 0.84 \\
0.00 & 0.00 & 0.00
\end{tabular}
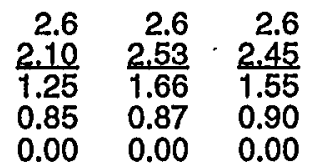

2.6
2.51
1.63
0.88

2.6
2.88
2.00

2.00
0.88

0.88
0.00

\begin{tabular}{r}
2.6 \\
3.16 \\
\hline 2.27 \\
0.89 \\
0.00
\end{tabular}

2.6
2.99
2.10
0.89

0.00

5.0

\begin{tabular}{rr}
5.0 & 5.0 \\
1.99 & 1.93 \\
\hline 1.26 & 1.22 \\
0.73 & 0.71 \\
0.00 & 0.00
\end{tabular}

\begin{tabular}{r}
5.0 \\
1.88 \\
\hline 1.15 \\
0.73 \\
0.00
\end{tabular}

5.0
2.04
1.29
0.75
0.00

5.0
2.51
1.76
0.75
0.00

5.0
2.39
1.60
0.79
0.00

5.0
2.46
1.68
0.78
0.00

5.0
2.76
1.98
0.78
0.00

\begin{tabular}{r}
5.0 \\
3.15 \\
\hline 2.36 \\
0.79 \\
0.00
\end{tabular}

5.0
2.80
2.01
0.79

$\frac{3.22}{2.43}$

$\frac{3.22}{2.43}$

0.79

5.0
2.36
1.57
0.79

0.79
0.00

6.0

\begin{tabular}{rr}
6.0 & 6.0 \\
1.99 & 1.93 \\
\hline 1.26 & 1.22 \\
0.73 & 0.71 \\
0.00 & 0.00
\end{tabular}

\begin{tabular}{r}
6.0 \\
1.88 \\
\hline 1.15 \\
0.73 \\
0.00
\end{tabular}

\begin{tabular}{r}
6.0 \\
2.04 \\
\hline 1.29 \\
0.75 \\
0.00
\end{tabular}

6.0
2.51
1.76
0.75
0.00

6.0
2.39
1.60
0.79
0.00

6.0
2.46
1.68
0.78
0.00

\begin{tabular}{r}
6.0 \\
2.76 \\
\hline 1.98 \\
0.78 \\
0.00
\end{tabular}

\begin{tabular}{r}
6.0 \\
3.15 \\
\hline 2.36 \\
0.79 \\
0.00
\end{tabular}

\begin{tabular}{r}
6.0 \\
2.80 \\
\hline 2.01 \\
0.79 \\
0.00
\end{tabular}

\begin{tabular}{r}
3.8 \\
3.22 \\
\hline 2.43 \\
0.79
\end{tabular}

6.0
2.36
1.57
0.79
0.00
4Q99

TQTAL Mc

84,284

386,041

382,493

$1,823,933$

405,000

$1,770,000$

486,000

$2,124,000$ 


\section{VOLUME AND PRICE REPORT}

\section{Long-Term Imports}

Volumes (MMcf/d) \& Prices (\$MMBTU) of Natural

Gas Imported During the Past 12 Months 01/01/99 - 12/31/99

Leng-Term Importer

Auth. $\quad 1999 \quad 1999 \quad 1999 \quad 1999$

Vols. Jan $\quad 1990.1999$

30.6

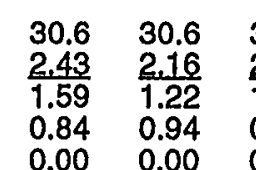

30.6
2.10
1.26
0.84
0.00

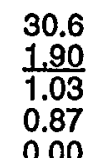

30.6
1.90
1.03
0.87
0.00

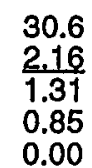

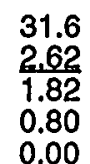

9.8

$\begin{array}{rrr}9.1 & 10.0 & 10.1 \\ \frac{1.56}{1.56} & \frac{1.55}{1.55} & 1.37 \\ 0.00 & 0.00 & 0.00 \\ 0.00 & 0.00 & 0.00\end{array}$

1.34
1.34
0.00

9.9
1.50

$\frac{1.50}{1.50}$

(Shell Canada Limited)

AVG Daily Quantity
Total Price (Port of Morgan. MT)

Commodity Component

Demand Component
Reservation Fee Component

$0.00 \quad 0.00$

$0.00 \quad 0.00$

CORAL ENERGY RESOURCES, L.P.-MIDAMERICAN ENERGY CO (2) (Shell Canada Limited)

AVG Daily Quantity

Total Price (Port of Morgan. MT)

Commodity Componen

Demand Component

Reservation Fee Component

20.5

CRESTAR ENERGY MARKETING CORPORATION (3)

(Crestar Energy)

AVG Daily Quantity

Total Price (Noyes, Minnesota)

15.0

Demand Component

Demand Component

Reservation Fee Component

CRESTAR ENERGY MARKETING CORPORATION (4)

(Crestar Energy)

AVG Daily Quantity $\quad 6.3$

Total Price (Port of Morgan, MT)

Commodity Component

Demand Component

Reservation Fee Component

$\begin{array}{rrr}10.3 & 9.9 & 9.9 \\ 1.86 & 1.81 & 1.86 \\ 1.86 & 1.81 & 1.86 \\ 0.00 & 0.00 & 0.00\end{array}$

$\begin{array}{lll}0.00 & 0.00 & 0.00\end{array}$ $\begin{array}{ll}1999 & 1999 \\ \text { Aug. } & \text { Sep }\end{array}$

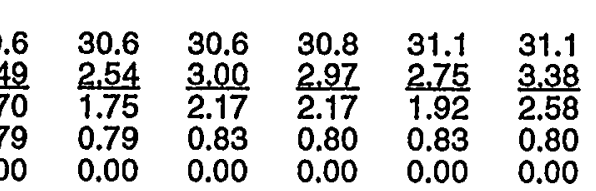

$2,849,234$

$11,232,554$

908,710

$3,612,644$

$\frac{2.10}{2.10}$

2.10

0.00
0.00

10.0
2.31
2.31
0.00

1.83

1.83

0.00

$\frac{2.84}{2.84}$

0.00

9.7
$\frac{2.12}{2.12}$

0.00

0.00

$\begin{array}{lll}20.7 & 20.4 & 19.7 \\ 1.56 & 1.55 & 1.34 \\ 1.56 & \frac{1.55}{1.34} \\ 0.00 & 0.00 & 0.00 \\ 0.00 & 0.00 & 0.00\end{array}$

\section{7}

1.50

1.50
0.00

0.00
0.00

$\begin{array}{ll}14.7 & 14.7 \\ 2.00 & 2.0 \\ 1.48 & 1.48 \\ 0.46 & 0.49 \\ 0.06 & 0.0\end{array}$

\begin{tabular}{ll}
14.7 & 14.7 \\
1.76 & 1.96 \\
\hline 1.24 & 1.43 \\
0.46 & 0.47 \\
0.06 & 0.06
\end{tabular}

$\begin{array}{lll}20.0 & 20.7 & 20.7 \\ 1.86 & \frac{1.81}{1.81} & 1.86 \\ 1.86 \\ 0.00 & 0.00 & 0.00 \\ 0.00 & 0.00 & 0.00\end{array}$

\section{4}

$\frac{2.10}{2.10}$

0.00
0.00

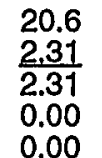

20.6

$\frac{1.83}{1.83}$

1.83

0.00
0.00

16.7
2.84

$\frac{2.84}{2.84}$

0.00

20.9

$\frac{2.12}{2.12}$

0.00
0.00
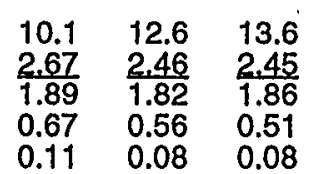

9.5
$\frac{3.07}{2.20}$
0.73
0.14

12.5
$\frac{3.17}{2.46}$
0.59

14.2
2.75
2.14
0.52
0.09

$\begin{array}{ll}10.6 & 14.6 \\ \frac{3.57}{2.74} & 2.44 \\ 0.68 & 0.49 \\ 0.15 & 0.08\end{array}$

$\begin{array}{ll}0.15 & 0.08\end{array}$

4.6
.44
.87
.49
.08

$1,210,641$

$4,758,684$

$1,789,673$

$7,337,633$

$\begin{array}{rrrrrrr}6.3 & 6.3 & 6.2 & 6.3 & 6.3 & 6.4 & 6.4 \\ .1 .96 & \frac{1.96}{1.64} & \frac{1.71}{1.39} & \frac{1.91}{1.59} & \frac{2.43}{2.11} & \frac{2.30}{1.98} & \frac{2.35}{2.03} \\ 0.32 & 0.32 & 0.32 & 0.32 & 0.32 & 0.32 & 0.32 \\ 0.00 & 0.00 & 0.00 & 0.00 & 0.00 & 0.00 & 0.00\end{array}$

6.3
$\frac{2.68}{2.36}$
0.32
0.00

6.4
$\frac{2.95}{2.63}$
0.32
0.00

6.4
2.70
2.38
0.32

5.1
$\frac{3.07}{2.74}$
0.33

6.4
$\frac{2.26}{1.94}$

1.94

0.32
0.00

(1) Successor to Salmon Resources Lid. (See DOE/FE order 690-A).

3 Supply is sold to Northern Slates Power Company for retail customers in the Grand Forks and Fargo areas of North Dakota. 
VOLUME AND PRICE REPORT

\section{Long-Term Imports}

Volumes (MMcf/d) \& Prices (\$MMBTU) of Natural

Gas Imported During the Past 12 Months 01/01/99 - 12/31/99
Long-Term Importer

DARTMOUTH POWER ASSOCIATES, L.P.

(Anderson Exploration Ltd.)

AVG Daily Quantity

Total Price (Waddington, NY)

Commodity Component

Demand Component

Reservation Fee Component

DARTMOUTH POWER ASSOCIATES, L.P.

(Crestar Energy Inc.)

AVG Daily Quantity

Total Price (Waddington. NY)

Commodity Component

Demand Component .

Reservation Fee Component

Auth.

Auth
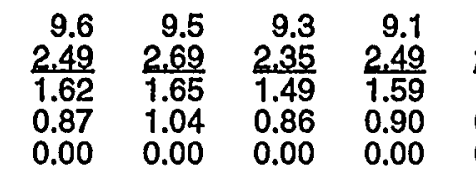

9.7
2.70
1.80
0.90
0.00

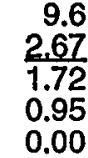

11.2
2.66
1.66
1.00
0.00

2.0

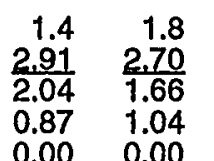

1.5
2.35
1.49
0.86
0.00

1.7
2.43
1.53
0.90
0.00

0.9
2.62
1.72
0.90
0.00

1.1
2.58
1.63
0.95
0.00
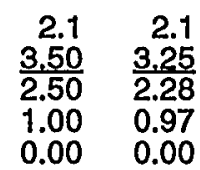

\begin{tabular}{r}
3.4 \\
3.91 \\
\hline 2.21 \\
1.70 \\
0.00
\end{tabular}

0.8
2.79
1.69
1.10

DARTMOUTH POWER ASSOCIATES, L.P.

(Remington Energy Ltd.)

AVG Daily Quantity

Total Price (Waddington. NY)

Commodity Component

Demand Component

Reservation Fee Component

DISTRIGAS CORPORATION (88-37-LNG) (1)

(Sonatrach)

AVG Daily Quantity

Total Price (Everett, Massachusetts)

Commodity Component

Demand Component

Reservation Fee Component

3.0

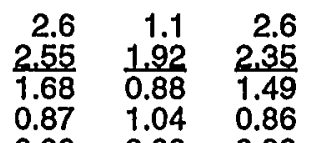

2.6
$\frac{2.45}{1.55}$

0.87

0.00

0.86

0.0

\begin{tabular}{rr}
339.2 & 188.9 \\
2.29 & 2.46 \\
\hline 2.29 & 2.46 \\
0.00 & 0.00
\end{tabular}

\begin{tabular}{r}
0.0 \\
0.00 \\
\hline 0.00 \\
0.00
\end{tabular}

168.7

$\frac{2.25}{2.25}$

0.00

0.00
0.00

2.7
2.70
1.80

1.80

0.90

2.7
2.67
1.72

1.72
0.95

$\begin{array}{r}0.9 \\ 3.29 \\ \hline\end{array}$

$\frac{3.29}{2.29}$

1.00

2.85

1.88
0.97

0.97
0.00

\begin{tabular}{r}
5.1 \\
3.91 \\
\hline 2.21 \\
1.70 \\
0.00
\end{tabular}

$\begin{array}{r}0.6 \\ 3.24 \\ \hline\end{array}$

$\frac{3.24}{2.14}$

1.10

0.00

$\begin{array}{r}0.0 \\ 0.00 \\ \hline .00\end{array}$

45.7

$\frac{1.91}{1.91}$

0.0

$\frac{0.00}{0.00}$

0.00
0.00

0.00
0.00
0.00

$\frac{0.00}{0.00}$

0.00
0.00

82.5

$\frac{2.36}{2.36}$

80.7

$\frac{2.58}{2.58}$

0.00

0.00

0.00

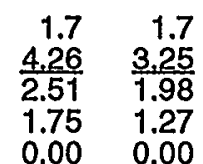

DISTRIGAS CORPORATION (89-16-LNG) (2)

(Sonatrach)

AVG Daily Quantity

Total Price (Everett, Massachusetts)

0.0

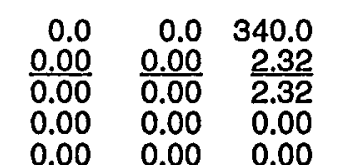

\begin{tabular}{r}
0.0 \\
0.00 \\
\hline 0.00 \\
0.00 \\
0.00
\end{tabular}

\begin{tabular}{l}
47.2 \\
1.97 \\
\hline 1.97 \\
0.00 \\
0.00
\end{tabular}

\begin{tabular}{lr}
48.8 & 0.0 \\
2.07 & 0.00 \\
\hline 2.07 & 0.00 \\
0.00 & 0.00 \\
0.00 & 0.00
\end{tabular}

81.3
2.63
2.63

$\frac{2.63}{2.63}$

0.00

2.63

2.63

0.00

Demand Component

$\begin{array}{llllllllllllll}0.00 & 0.00 & 0.00 & 0.00 & 0.00 & 0.00 & 0.00 & 0.00 & 0.00 & 0.00 & 0.00 & 0.00\end{array}$

129,219

612,096

$4 Q 99$

IQTAL Mcf

517,971

$3,079,716$

YTD
TOTALMCf

176,864

832,613

$7,435,270$

$32,193,657$

0

$13,468,289$

(1) Landed Price

(2) Landed Price. (The May and June volumes originated in Trinidad; the Algerian LNG volumes were delivered to Spain in a swap cargo arrangement.) 


\section{VOLUME AND PRICE REPORT}

\section{Long-Term Imports}

Volumes (MMcf/d) \& Prices (\$MMBTU) of Natural

Gas Imported During the Past 12 Months $01101 / 99$ - 12/31/99

\begin{tabular}{|c|c|c|c|c|c|c|c|c|c|c|c|c|c|c|c|}
\hline Leng-Term Importer & $\begin{array}{l}\text { Auth. } \\
\text { Vols. }\end{array}$ & $\begin{array}{l}1999 \\
\text { Jan. }\end{array}$ & $\begin{array}{l}1999 \\
\text { Eeb. }\end{array}$ & $\begin{array}{l}1999 \\
\text { Mar. }\end{array}$ & $\begin{array}{l}1999 \\
\text { Apr. }\end{array}$ & $\begin{array}{l}1999 \\
\text { May. }\end{array}$ & $\begin{array}{l}1999 \\
\text { Jun. }\end{array}$ & $\begin{array}{l}1999 \\
\text { Jul. }\end{array}$ & $\begin{array}{l}1999 \\
\text { Aug. }\end{array}$ & $\begin{array}{l}1999 \\
\text { Sep. }\end{array}$ & $\begin{array}{l}1999 \\
\text { Qct. }\end{array}$ & $\begin{array}{l}1999 \\
\text { Nov. }\end{array}$ & $\begin{array}{l}1999 \\
\text { Dec. }\end{array}$ & $\begin{array}{l}\text { 4099 } \\
\text { IOTAL Mcf }\end{array}$ & $\begin{array}{c}\text { YTD } \\
\text { IOTALMaf }\end{array}$ \\
\hline $\begin{array}{l}\text { DISTRIGAS CORPORATION (95-100- } \\
\text { (Cabot LNG Trading Limited) } \\
\text { AVG Daily Quantity } \\
\text { Total Price (Everett. Massachusetts) } \\
\text { Commodity Component } \\
\text { Demand Component } \\
\text { Reservation Fee Component }\end{array}$ & 0.0 & $\begin{array}{r}0.0 \\
0.00 \\
0.00 \\
0.00 \\
0.00\end{array}$ & $\begin{array}{r}0.0 \\
0.00 \\
0.00 \\
0.00 \\
0.00\end{array}$ & $\begin{array}{r}0.0 \\
0.00 \\
0.00 \\
0.00 \\
0.00\end{array}$ & $\begin{array}{r}0.0 \\
0.00 \\
0.00 \\
0.00 \\
0.00\end{array}$ & $\begin{array}{l}82.8 \\
1.63 \\
1.63 \\
0.00 \\
0.00\end{array}$ & $\begin{array}{r}171.9 \\
1.96 \\
1.96 \\
0.00 \\
0.00\end{array}$ & $\begin{array}{r}212.9 \\
2.01 \\
2.01 \\
0.00 \\
0.00\end{array}$ & $\begin{array}{r}177.8 \\
2.34 \\
2.34 \\
0.00 \\
0.00\end{array}$ & $\begin{array}{l}97.5 \\
2.55 \\
2.55 \\
0.00 \\
0.00\end{array}$ & $\begin{array}{l}47.3 \\
2.79 \\
2.79 \\
0.00 \\
0.00\end{array}$ & $\begin{array}{r}172.4 \\
2.74 \\
2.74 \\
0.00 \\
0.00\end{array}$ & $\begin{array}{r}169.5 \\
2.70 \\
2.70 \\
0.00 \\
0.00\end{array}$ & $11,894,742$ & $34,655,622$ \\
\hline $\begin{array}{l}\text { DUKE ENERGY LNG SALES, INC. (2) } \\
\text { (Sonatrading) } \\
\text { AVG Daily Quantity } \\
\text { Total Price (Lake Charles, LA) } \\
\text { Commodity Component } \\
\text { Demand Component } \\
\text { Reservation Fee Component }\end{array}$ & 123.3 & $\begin{array}{l}73.4 \\
2.00 \\
2.00 \\
0.00 \\
0.00\end{array}$ & $\begin{array}{l}68.7 \\
2.00 \\
2.00 \\
0.00 \\
0.00\end{array}$ & $\begin{array}{l}73.7 \\
2.00 \\
2.00 \\
0.00 \\
0.00\end{array}$ & $\begin{array}{l}70.7 \\
2.00 \\
2.00 \\
0.00 \\
0.00\end{array}$ & $\begin{array}{l}76.6 \\
2.00 \\
2.00 \\
0.00 \\
0.00\end{array}$ & $\begin{array}{l}75.9 \\
2.00 \\
2.00 \\
0.00 \\
0.00\end{array}$ & $\begin{array}{l}78.4 \\
2.00 \\
2.00 \\
0.00 \\
0.00\end{array}$ & $\begin{array}{l}78.7 \\
2.00 \\
2.00 \\
0.00 \\
0.00\end{array}$ & $\begin{array}{l}77.2 \\
2.00 \\
2.00 \\
0.00 \\
0.00\end{array}$ & $\begin{array}{l}79.8 \\
2.00 \\
2.00 \\
0.00 \\
0.00\end{array}$ & $\begin{array}{r}0.0 \\
0.00 \\
0.00 \\
0.00 \\
0.00\end{array}$ & $\begin{array}{r}0.0 \\
0.00 \\
0.00 \\
0.00 \\
0.00\end{array}$ & $2,472,781$ & $22,913,743$ \\
\hline $\begin{array}{l}\text { ELIZABETHTOWN GAS COMPANY } \\
\text { (TCGS LId.) } \\
\text { AVG Daily Quantity } \\
\text { Total Price (Niagara Falls, NY) } \\
\text { Commodity Component } \\
\text { Demand Component } \\
\text { Reservation Fee Component }\end{array}$ & 10.0 & $\begin{array}{l}10.0 \\
2.95 \\
2.02 \\
0.93 \\
0.00\end{array}$ & $\begin{array}{l}10.1 \\
2.95 \\
1.95 \\
1.00 \\
0.00\end{array}$ & $\begin{array}{r}5.8 \\
3.53 \\
1.88 \\
1.65 \\
0.00\end{array}$ & $\begin{array}{l}10.0 \\
2.49 \\
1.51 \\
0.98 \\
0.00\end{array}$ & $\begin{array}{l}10.0 \\
2.58 \\
1.62 \\
0.96 \\
0.00\end{array}$ & $\begin{array}{l}10.0 \\
2.64 \\
1.60 \\
1.04 \\
0.00\end{array}$ & $\begin{array}{l}10.0 \\
2.79 \\
1.78 \\
1.01 \\
0.00\end{array}$ & $\begin{array}{l}10.0 \\
2.95 \\
1.96 \\
0.99 \\
0.00\end{array}$ & $\begin{array}{l}10.0 \\
3.28 \\
2.25 \\
1.03 \\
0.00\end{array}$ & $\begin{array}{l}10.0 \\
3,33 \\
2.33 \\
1.00 \\
0.00\end{array}$ & $\begin{array}{l}10.0 \\
3.00 \\
1.97 \\
1.03 \\
0.00\end{array}$ & $\begin{array}{l}10.0 \\
3.03 \\
2.03 \\
1.00 \\
0.00\end{array}$ & 920,000 & $3,523,689$ \\
\hline $\begin{array}{l}\text { ENCOGEN NORTHWEST, L.P. (3) } \\
\text { (CanWest Gas Supply, Inc.) } \\
\text { AVG Daily Quantity } \\
\text { Total Price (Sumas. Washington) } \\
\text { Commodity Component } \\
\text { Demand Component } \\
\text { Reservation Fee Component }\end{array}$ & 9.6 & $\begin{array}{r}7.8 \\
2.35 \\
2.35 \\
0.00 \\
0.00\end{array}$ & $\begin{array}{r}7.9 \\
2.35 \\
2.35 \\
0.00 \\
0.00\end{array}$ & $\begin{array}{r}0.0 \\
0.00 \\
0.00 \\
0.00 \\
0.00\end{array}$ & $\begin{array}{r}1.0 \\
2.35 \\
2.35 \\
0.00 \\
0.00\end{array}$ & $\begin{array}{r}6.9 \\
2.35 \\
2.35 \\
0.00 \\
0.00\end{array}$ & $\begin{array}{r}9.0 \\
2.35 \\
2.35 \\
0.00 \\
0.00\end{array}$ & $\begin{array}{r}0.0 \\
0.00 \\
0.00 \\
0.00 \\
0.00\end{array}$ & $\begin{array}{r}7.6 \\
2.35 \\
2.35 \\
0.00 \\
0.00\end{array}$ & $\begin{array}{r}7.5 \\
2.35 \\
2.35 \\
0.00 \\
0.00\end{array}$ & $\begin{array}{r}0.0 \\
0.00 \\
0.00 \\
0.00 \\
0.00\end{array}$ & $\begin{array}{r}0.0 \\
0.00 \\
0.00 \\
0.00 \\
0.00\end{array}$ & $\begin{array}{r}0.0 \\
0.00 \\
0.00 \\
0.00 \\
0.00\end{array}$ & 0 & $1,436,765$ \\
\hline $\begin{array}{l}\text { ENERGYNORTH, INC. } \\
\text { (Alberta NE Gas (TCGS Ltd. 1)) } \\
\text { AVG Daily Quantity } \\
\text { Total Price (Waddington, NY) } \\
\text { Commodity Component } \\
\text { Demand Component } \\
\text { Reservation Fee Component }\end{array}$ & 4.0 & $\begin{array}{r}4.0 \\
1.99 \\
1.26 \\
0.73 \\
0.00\end{array}$ & $\begin{array}{r}4.0 \\
1.93 \\
1.22 \\
0.71 \\
0.00\end{array}$ & $\begin{array}{r}4.0 \\
1.88 \\
1.15 \\
0.73 \\
0.00\end{array}$ & $\begin{array}{r}4.0 \\
2.04 \\
1.29 \\
0.75 \\
0.00\end{array}$ & $\begin{array}{r}4.0 \\
2.51 \\
1.76 \\
0.75 \\
0.00\end{array}$ & $\begin{array}{r}4.0 \\
2.39 \\
1.60 \\
0.79 \\
0.00\end{array}$ & $\begin{array}{r}4.0 \\
2.46 \\
1.68 \\
0.78 \\
0.00\end{array}$ & $\begin{array}{r}4.0 \\
2.76 \\
1.98 \\
0.78 \\
0.00\end{array}$ & $\begin{array}{r}4.0 \\
\frac{3.15}{2.36} \\
0.79 \\
0.00\end{array}$ & $\begin{array}{r}4.0 \\
2.80 \\
2.01 \\
0.79 \\
0.00\end{array}$ & $\begin{array}{r}2.9 \\
3.22 \\
2.43 \\
0.79 \\
0.00\end{array}$ & $\begin{array}{r}4.0 \\
2.36 \\
1.57 \\
0.79 \\
0.00\end{array}$ & 336,000 & $1,428,000$ \\
\hline
\end{tabular}

(1) Under a 20-year contract, Cabot LNG Trading Ltd. will purchase up to 83,000,000 MMBtu of LNG from Atlantic LNG Co. of Trinidad and Tobago. Landed Price.

(3) Supply used to fuel a 160 Megawatt gas-fired cogeneration facility located at Bellingham. Washington. 


\section{VOLUME AND PRICE REPORT}

\section{Long-Term Imports}

Volumes (MMcf/d) \& Prices ( $\$ M M B T U$ ) of Natura

Gas Imported During the Past 12 Months 01/01/99 - 12/31/99

Long-Term Importer

$\begin{array}{lllll}\text { Auth. } & 1999 & 1999 & 1999 & 1999\end{array}$

19991999

Vols.

ENGAGE ENERGY CANADA, L.P. - CIBOLA CORP.

AVG Daily Quantity

Total Price (Port of Morgan. MT)

1.2

Commodity Component

Reservation Fee Component

$\begin{array}{rrrr}1.2 & 1.2 & 1.2 & 1.2 \\ \frac{1.70}{1.70} & \frac{1.65}{1.65} & \frac{1.45}{1.45} & \frac{1.60}{1.60} \\ 0.00 & 0.00 & 0.00 & 0.00 \\ 0.00 & 0.00 & 0.00 & 0.00\end{array}$

Jan. Feb. Mar. Apr.
May.

1.2
2.05
2.05
0.00
0.00

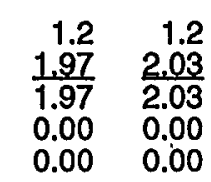

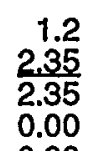

1.2
2.35
2.35
0.00
0.00

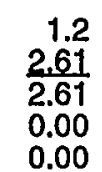

ENGAGE ENERGY CANADA, L.P. - I'STATE POWER CO. (1)

(Engage Energy Canada, L.P.)

AVG Daily Quantity

Total Price (Port of Morgan. MT)

1.0

Commodity Component

Demand Component

Reservation Fee Component

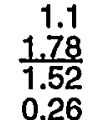

1.1

1.1
1.57
1.34

1.1
$\frac{1.77}{1.50}$

1.1
2.18

1.51

0.29
0.00

0.23
0.00

0.27
0.00

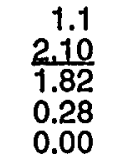

1.1
1.92
1.66
0.26

1.1
2.42
2.15

2.42
2.15
0.27
0.00

1.1
2.66
2.38
0.28

ENGAGE ENERGY CANADA, L.P. - METRO. UTIL. DIS

(Engage Energy Canada, L.P.)

AVG Daily Quantity

Total Price (Port of Morgan, MT)

3.7

Commodity Component

Reservation Fee Component

3.8
1.76
1.58
0.18
0.00

\begin{tabular}{r}
3.8 \\
1.75 \\
\hline 1.56 \\
0.19
\end{tabular}

3.8
1.56
1.38
0.18
0.00

\begin{tabular}{r}
3.8 \\
1.73 \\
\hline 1.55 \\
0.18 \\
0.00
\end{tabular}

3.8
2.18
2.00
0.18
0.00

3.8
2.09
1.91
0.18
0.00

3.8
2.14
1.96
0.18
0.00

3.8
2.55
2.37
0.18
0.00

3.8
2.70
2.52
0.18

0.18

$\frac{2.29}{2.20}$

0.09

$\frac{3.03}{2.70}$

2.70
0.33

0.00

1.97

1.86

0.11

ENGAGE ENERGY CANADA, L.P. - MIDAMERICAN

(Engage Energy Canada, L.P.)
6.7
2.29
2.15
0.14
0.00
AVG Daily Quantity

Total Price (Port of Morgan. MT)

6.5

Commodity Component

Demand Component

Reservation Fee Component

\begin{tabular}{rr}
6.7 & 6.7 \\
1.66 & 1.66 \\
\hline 1.52 & 1.51 \\
0.14 & 0.15
\end{tabular}

6.7

6.5

6.6

6.7
1.96
1.82

6.7
2,02
1.88

0.00

0.00

0.14

0.14

0.00

0.14

0.00

0.14

0.00
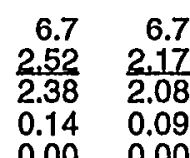

$\frac{2.17}{2.08}$

0.09

ENGAGE ENERGY CANADA, L.P. - N'WESTERN PUB.

(Engage Energy Canada, L.P.)

AVG Daily Quantity

Total Price (Port of Morgan. MT)

0.9

\begin{tabular}{r}
1.0 \\
1.76 \\
\hline 1.58 \\
0.18
\end{tabular}

\begin{tabular}{r}
0.9 \\
1.76 \\
\hline 1.56 \\
0.20
\end{tabular}

\begin{tabular}{r}
1.0 \\
1.56 \\
\hline 1.38 \\
0.18 \\
0.00
\end{tabular}

1.0
1.73
1.55
0.18
0.00

1.0
2.17
1.99
0.18
0.00

1.0
2.09
1.91
0.18
0.00

\begin{tabular}{r}
1.0 \\
2.46 \\
\hline 2.28 \\
0.18 \\
0.00
\end{tabular}

Reservation Fee Component \begin{tabular}{r}
1.0 \\
2.14 \\
\hline 1.96 \\
0.18 \\
0.00
\end{tabular}

$4 Q 99$

TOTAL MCf

YTD
TOTALMCf

(1) Contract with Interstale Power Company terminated 10/31/99. 
VOLUME AND PRICE REPORT

Long-Term Imports

Volumes (MMcf/d) \& Prices (\$MMBTU) of Natural

Gas Imported During the Past 12 Months 01/01/99 - 12/31/99
Long-Term Importer

Auth

Auth.
Vols.
1999
Jan

ENGAGE ENERGY CANADA, L.P. - WISCONSIN GAS

(Engage Energy Canada, L.P.)

AVG Daily Quantity

Total Price (Port of Morgan. MT)

Commodity Component

Reservation Fee Component

2.4

$\begin{array}{rrr}2.5 & 2.5 & 2.5 \\ 1.69 & 1.67 & 1.49 \\ 1.55 & \frac{1.52}{1.35} \\ 0.14 & 0.15 & 0.14 \\ 0.00 & 0.00 & 0.00\end{array}$

2.5
1.67
1.52
0.15
0.00

2.11

$\frac{2.11}{1.96}$

0.00

48.3

$\begin{array}{ll}10.4 & 1 \\ 2.31 & 2 \\ 2.31 & 2 \\ 0.00 & 0.00 \\ 0.00 & 0.00\end{array}$

$\begin{array}{rr}14.5 & 8.5 \\ 2.17 & 2.24 \\ 2.17 & 2.24 \\ 0.00 & 0.00 \\ 0.00 & 0.00\end{array}$

11.5

$\frac{2.17}{2.17}$

2.17
0.00

$0.00 \quad 0.00$

Reservation Fee Component

ENGAGE ENERGY U.S., L.P. (3)

(Morrison Petroleums Ltd.)

AVG Daily Quantity

Total Price (Noyes, Minnesota)

Commodity Component

Demand Component

Reservation Fee Component

ENGAGE ENERGY U.S., L.P. (4)

(Petro-Canada Resources)

AVG Daily Quantity

Total Price (Noyes, Minnesota)

Commodity Componen

Demand Component

Reservation Fee Component

\begin{tabular}{llll}
11.2 & 11.2 & 11.2 & 11.2 \\
1.76 & $\frac{1.71}{1.71}$ & $\frac{1.55}{1.55}$ & $\frac{1.65}{1.65}$ \\
\hline 1.76 & 1.71 & 0.00 & 0.00 \\
0.00 & 0.00 & 0.00 & 0.00 \\
0.00 & 0.00 & 0.00 & 0.00
\end{tabular}

10.0

$\begin{array}{lll}11.0 & 11.2 & 11.1 \\ 2.21 & 2.17 & 1.82 \\ 2.21 & 2.17 & 1.82 \\ 0.00 & 0.00 & 0.00 \\ 0.00 & 0.00 & 0.00\end{array}$

10.2
2.06
2.06
0.00
0.00

2.55

$\frac{2.55}{2.55}$

0.00

0.00

10.2
2.37
2.37
0.00

10.1

$\frac{2.39}{2.39}$

0.00

10.2
2.79
2.79
0.00
0.00

10.2
3.12
3.12
0.00

10.2
2.76
2.76
0.00

\begin{tabular}{l}
10.2 \\
3.42 \\
\hline 3.42
\end{tabular}

3.42

0.00

10.1
2.57
2.57
0.00
0.00
7.0

\begin{tabular}{rrrr}
7.1 & 7.1 & 7.1 & 7.1 \\
1.76 & $\frac{1.71}{1.71}$ & $\frac{1.55}{1.55}$ & $\frac{1.65}{1.65}$ \\
\hline .00 & 0.00 & 0.00 & 0.00 \\
0.00 & 0.00 & 0.00 & 0.00
\end{tabular}

$\begin{array}{rrr}7.1 & 7.1 & 7.1 \\ 2.11 & 2.07 & 2.04 \\ 2.11 & 2.07 & 2.04 \\ 0.00 & 0.00 & 0.00 \\ 0.00 & 0.00 & 0.00\end{array}$

$\begin{array}{lll}0.00 & 0.00 & 0.00\end{array}$
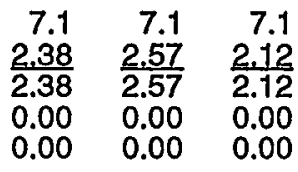

7.2
2.50
2.50
0.00

7.2
$\frac{1.76}{1.76}$
0.00

11.1

$\begin{array}{lll}11.2 & 11.2 & 11.2 \\ \frac{2.11}{2.11} & \frac{2.07}{2.07} & \frac{2.04}{2.04} \\ 0.00 & 0.00 & 0.00 \\ 0.00 & 0.00 & 0.00\end{array}$

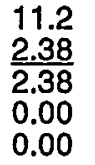

11.2
2.57
2.57
0.00
0.00

11.2
2.12
2.12
0.00
0.00

11.2
2.51
2.51
0.00
0.00

\begin{tabular}{l}
11.2 \\
1.76 \\
\hline 1.76 \\
0.00 \\
0.00
\end{tabular}

$1,029,016$

$4,085,781$

$\left(\begin{array}{l}1 \\ 2 \\ 3 \\ 4\end{array}\right)$

These volumes were delivered al Niagara Falls, NY: July (155,000 Mcf); August (155,000 Mcf); Seplember (155,000 Mcf).

Supplies will serve CGM's markels in the U.S, northeast, including CGM's Eagle Point Refinery in Westville, New Jersey.

Supplies sold to ProGold L.L.C. for its com milling facilities in North Dakota.
4) Supplies sold to American Crystal Sugar for its refineries in Minnesota.: 


\section{VOLUME AND PRICE REPORT}

\section{Long-Term Imports}

Volumes (MMct/d) \& Prices (\$MMBTU) of Natural

Gas Imported During the Past 12 Months 01/01/99 - 12/31/99

\section{Long-Term Importer}

ENGAGE ENERGY U.S., L.P. (1)

(Rio Alto, Pinnacle, Jordan)

AVG Daily Quantity

Total Price (Waddington. NY)

Commodity Component

Reservation Fee Component

ENGAGE ENERGY U.S., L.P. (2)

(Various Suppliers)

A Total Pric (uantity Minnesota)

Total Price. Noyes. Minnes

Demand Component

Reservation Fee Component

ENGAGE ENERGY U.S., L.P.

(Various Suppliers)

AVG Daily Quantity

Total Price (St, Clair. Michigan)

Commodity Component

Demand Component

Reservation Fee Component

ENGAGE ENERGY U.S., L.P.

(Various Suppliers)

Total Price (Noves, Minnesota)

Commodity Component

Demand Component

Reservation Fee Component

ENRON NORTH AMERICA CORP. (3)

\section{(ECTR - Canada)}

AVG Daily Quantity

Total Price (Waddington. NY)

Commodity Component

Demand Component

Reservation Fee Component

\begin{tabular}{|c|c|c|c|c|c|c|c|c|c|c|c|}
\hline $\begin{array}{l}\text { Auth. } \\
\text { Vols. }\end{array}$ & $\begin{array}{l}1999 \\
\text { Jan. }\end{array}$ & $\begin{array}{l}1999 \\
\text { Feb. }\end{array}$ & $\begin{array}{l}1999 \\
\text { Mar. }\end{array}$ & $\begin{array}{l}1999 \\
\text { Apr. }\end{array}$ & $\begin{array}{l}1999 \\
\text { May. }\end{array}$ & $\begin{array}{c}1999 \\
\text { Jun. }\end{array}$ & $\begin{array}{l}1999 \\
\text { Jul. }\end{array}$ & $\begin{array}{l}1999 \\
\text { Aug. }\end{array}$ & $\begin{array}{l}1999 \\
\text { Sep. }\end{array}$ & $\begin{array}{l}1999 \\
\text { Qct. }\end{array}$ & $\begin{array}{l}1999 \\
\text { Nov. }\end{array}$ \\
\hline 14.0 & $\begin{array}{l}10.2 \\
2.21 \\
2.21 \\
0.00 \\
0.00\end{array}$ & $\begin{array}{l}10.1 \\
2.17 \\
2.17 \\
0.00 \\
0.00\end{array}$ & $\begin{array}{r}9.2 \\
1.87 \\
1.87 \\
0.00 \\
0.00\end{array}$ & $\begin{array}{r}9.2 \\
2.06 \\
2.06 \\
0.00 \\
0.00\end{array}$ & $\begin{array}{r}9.2 \\
2.52 \\
2.52 \\
0.00 \\
0.00\end{array}$ & $\begin{array}{r}9.2 \\
2.37 \\
2.37 \\
0.00 \\
0.00\end{array}$ & $\begin{array}{r}9.1 \\
2.39 \\
2.39 \\
0.00 \\
0.00\end{array}$ & $\begin{array}{r}9.1 \\
2.79 \\
2.79 \\
0.00 \\
0.00\end{array}$ & $\begin{array}{r}9.1 \\
3.12 \\
3.12 \\
0.00 \\
0.00\end{array}$ & $\begin{array}{r}9.2 \\
2.76 \\
2.76 \\
0.00 \\
0.00\end{array}$ & $\begin{array}{r}9.2 \\
3.42 \\
3.42 \\
0.00 \\
0.00\end{array}$ \\
\hline 25.0 & $\begin{array}{l}31.1 \\
1.86 \\
1.86 \\
0.00 \\
0.00\end{array}$ & $\begin{array}{l}33.0 \\
1.90 \\
1.90 \\
0.00 \\
0.00\end{array}$ & $\begin{array}{l}14.6 \\
1.74 \\
1.74 \\
0.00 \\
0.00\end{array}$ & $\begin{array}{l}15.2 \\
1.99 \\
1.99 \\
0.00 \\
0.00\end{array}$ & $\begin{array}{l}15.2 \\
2.42 \\
2.42 \\
0.00 \\
0.00\end{array}$ & $\begin{array}{l}15.2 \\
2.31 \\
2.31 \\
0.00 \\
0.00\end{array}$ & $\begin{array}{l}15.2 \\
2.35 \\
2.35 \\
0.00 \\
0.00\end{array}$ & $\begin{array}{l}15.2 \\
2.70 \\
2.70 \\
0.00 \\
0.00\end{array}$ & $\begin{array}{l}15.2 \\
2.98 \\
2.98 \\
0.00 \\
0.00\end{array}$ & $\begin{array}{l}15.2 \\
2.64 \\
2.64 \\
0.00 \\
0.00\end{array}$ & $\begin{array}{l}15.2 \\
3.24 \\
3.24 \\
0.00 \\
0.00\end{array}$ \\
\hline 49.0 & $\begin{array}{l}15.2 \\
1.91 \\
1.91 \\
0.00 \\
0.00\end{array}$ & $\begin{array}{l}15.2 \\
1.92 \\
1.92 \\
0.00 \\
0.00\end{array}$ & $\begin{array}{l}15.2 \\
1.74 \\
1.74 \\
0.00 \\
0.00\end{array}$ & $\begin{array}{r}0.6 \\
2.30 \\
2.30 \\
0.00 \\
0.00\end{array}$ & $\begin{array}{l}45.0 \\
2.43 \\
2.43 \\
0.00 \\
0.00\end{array}$ & $\begin{array}{l}45.0 \\
2.31 \\
2.31 \\
0.00 \\
0.00\end{array}$ & $\begin{array}{l}45.0 \\
2.33 \\
2.33 \\
0.00 \\
0.00\end{array}$ & $\begin{array}{l}45.0 \\
2.69 \\
2.69 \\
0.00 \\
0.00\end{array}$ & $\begin{array}{l}42.0 \\
2.97 \\
2.97 \\
0.00 \\
0.00\end{array}$ & $\begin{array}{l}49.5 \\
2.63 \\
2.63 \\
0.00 \\
0.00\end{array}$ & $\begin{array}{l}45.0 \\
3.25 \\
3.25 \\
0.00 \\
0.00\end{array}$ \\
\hline 30.5 & $\begin{array}{l}19.7 \\
1.76 \\
1.76 \\
0.00 \\
0.00\end{array}$ & $\begin{array}{l}19.4 \\
1.71 \\
1.71 \\
0.00 \\
0.00\end{array}$ & $\begin{array}{l}28.0 \\
1.36 \\
1.36 \\
0.00 \\
0.00\end{array}$ & $\begin{array}{l}27.1 \\
1.83 \\
1.83 \\
0.00 \\
0.00\end{array}$ & $\begin{array}{l}30.3 \\
2.34 \\
2.34 \\
0.00 \\
0.00\end{array}$ & $\begin{array}{l}32.3 \\
2.22 \\
2.22 \\
0.00 \\
0.00\end{array}$ & $\begin{array}{l}30.0 \\
2.24 \\
2.24 \\
0.00 \\
0.00\end{array}$ & $\begin{array}{l}30.0 \\
2.59 \\
2.59 \\
0.00 \\
0.00\end{array}$ & $\begin{array}{l}30.0 \\
2.86 \\
2.86 \\
0.00 \\
0.00\end{array}$ & $\begin{array}{l}30.0 \\
2.51 \\
2.51 \\
0.00 \\
0.00\end{array}$ & $\begin{array}{l}30.9 \\
3.21 \\
3.21 \\
0.00 \\
0.00\end{array}$ \\
\hline 15.0 & $\begin{array}{l}2.0 \\
2.23 \\
2.23 \\
0.00 \\
0.00\end{array}$ & $\begin{array}{r}2.0 \\
1.39 \\
1.39 \\
0.00 \\
0.00\end{array}$ & $\begin{array}{r}2.0 \\
1.53 \\
1.53 \\
0.00 \\
0.00\end{array}$ & $\begin{array}{r}2.0 \\
1.77 \\
1.77 \\
0.00 \\
0.00\end{array}$ & $\begin{array}{r}2.0 \\
2.24 \\
2.24 \\
0.00 \\
0.00\end{array}$ & $\begin{array}{r}2.0 \\
2.12 \\
2.12 \\
0.00 \\
0.00\end{array}$ & $\begin{array}{r}2.0 \\
2.13 \\
2.13 \\
0.00 \\
0.00\end{array}$ & $\begin{array}{r}2.0 \\
2.48 \\
2.48 \\
0.00 \\
0.00\end{array}$ & $\begin{array}{r}2.0 \\
2.79 \\
2.79 \\
0.00 \\
0.00\end{array}$ & $\begin{array}{r}2.0 \\
2.44 \\
2.44 \\
0.00 \\
0.00\end{array}$ & $\begin{array}{l}2.0 \\
2.96 \\
2.96 \\
0.00 \\
0.00\end{array}$ \\
\hline
\end{tabular}

$4 Q 99$ TOTALMCF

842,174

$3,398,675$

$1,397,940$

$6,517,176$

$2,886,238$

$11,073,703$

$2,805,156$

$10,305,182$

184,000

730,000

(1) Some of these supplies are used via displacement, at the company's Eagle Point refinery in Westville. N.J.

(2) Exported under Engage's blanket export authorization at St. Clair, Ml: Jan $(310,000)$, Feb (337,652), Mar (945,004), Apr (16,470), May (148,924), and Jun (2,280). 


\section{VOLUME AND PRICE REPORT}

\section{Long-Term Imports}

Volumes (MMcf/d) \& Prices (\$MMBTU) of Natural Gas Imported During the Past 12 Months 01/01/99 - 12/31/99
Long-Term Importer

\section{ENRON NORTH AMERICA CORP. (1)}

(ECTR - Canada)

Total Price (Niagara Falls, NY)

Commodity Component

Demand Component

Reservation Fee Component

ENRON NORTH AMERICA CORP. (2)

AVG Daily Quantity

Total Price (Niagara Falls. NY)

Demand Component

Reservation Fee Component

ENRON NORTH AMERICA CORP. (3)

ECTR - Canada)

Total Price (Port of Morgan, MT)

Commodity Component

Demand Component

Reservation Fee Component

ENRON NORTH AMERICA CORP. (4)

(ECTR - Canada)

AVG Daily Quantity

Total Price (Noyes, Minnesota)

Commodity Component

Demand Component

Reservation Fee Component

ESSEX COUNTY GAS CO

(Alberta NE Gas (TCGS Ltd. 1))

AVG Daily Quantity

Total Price (Waddington. NY)

Commodity Componen

Reservation Fee Component
ECTR - Canada)

Commodity Componen

AVG Daily Quantity
Auth

1999

1999

1999

1999

1999

15.4

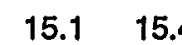

\begin{tabular}{ll}
1.87 & $\frac{1.95}{1.87}$ \\
\hline .95
\end{tabular}

$\begin{array}{ll}0.00 & 0.00 \\ 0.00 & 0.00\end{array}$

15.4
1.79
1.79
0.00

0.0
0.00
0.00

0.0

0.0

0.00

0.00

9.0

$\begin{array}{rrr}8.3 & 9.1 & 9.2 \\ \frac{1.90}{1.90} & \frac{1.92}{1.92} & \frac{1.73}{1.73} \\ 0.00 & 0.00 & 0.00\end{array}$

$0.00 \quad 0.00$

0.00

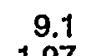

9.1
$\frac{1.97}{1.97}$

$\frac{1.97}{1.97}$
0.00

0.00

9.1
2.42
2.42

$\frac{2.42}{2.42}$

0.00
0.00

\begin{tabular}{rr}
9.1 & 9.1 \\
2.31 & 2.35 \\
\hline
\end{tabular}

$\frac{2.31}{2.31} \cdot \frac{2.35}{2.35}$

0.00

$0.00 \quad 0.00$

9.1
2.70
270

$\frac{2.70}{2.70}$

0.00
0.00

$\frac{2.97}{2.97}$

000

$0.00 \quad 0.00$

42.0

$\begin{array}{llll}40.0 & 40.0 & 40.0 & 40.0 \\ \frac{1.78}{1.78} & \frac{1.52}{1.52} & \frac{1.58}{1.58} & \frac{1.73}{1.73} \\ 0.00 & 0.00 & 0.00 & 0.00 \\ 0.00 & 0.00 & 0.00 & 0.00\end{array}$

40.0
2.21
2.21
0.00
0.00

40.0
2.08
2.08
0.00
0.00

40.0
2.21
2.21
0.00
0.00

40.0
2.64
2.64
0.00
0.00

40.0

$$
2.00
$$

\begin{tabular}{lll}
40.0 & 40.0 & 40.0 \\
2.60 & $\frac{3.16}{2.60}$ & 2.02 \\
\hline 2.16 & 2.02 \\
0.00 & 0.00 & 0.00 \\
0.00 & 0.00 & 0.00
\end{tabular}

$3,680,000$

$14,600,000$

30.4

$\begin{array}{llll}30.4 & 26.2 & 30.4 & 31.3 \\ \frac{1.90}{1.90} & \frac{1.92}{1.92} & \frac{1.73}{1.73} & \frac{1.98}{1.98} \\ 0.00 & 0.00 & 0.00 & 0.00\end{array}$

31.4
2.42
2.42
0.00

31.4
2.31
2.31
0.00

31.4
2.35
2.35
0.00

31.6
2.70
2.70
0.00

31.4
2.97
2.97
0.00
0.00

31.6
2.63
2.63
0.00
0.00

31.6
3.22
3.22
0.00

$\begin{array}{ll}0.00 & 0.00 \\ 0.00 & 0.00\end{array}$

$0.00 \quad 0.00$
0

2.0

$\begin{array}{rrrr}2.0 & 2.0 & 2.0 & 2.0 \\ \frac{1.99}{1.26} & \frac{1.93}{1.22} & \frac{1.88}{1.15} & \frac{2.04}{1.29} \\ 0.73 & 0.71 & 0.73 & 0.75 \\ 0.00 & 0.00 & 0.00 & 0.00\end{array}$

2.0
2.51
1.76
0.75
0.00

2.0
$\frac{2.39}{1.60}$
0.79
0.00

2.0
2.46
1.68
0.78
0.00

2.0
2.76
1.98
0.78
0.00

2.0
$\frac{3.15}{2.36}$
0.79
0.00

2.0
$\frac{2.80}{2.01}$
0.79
0.00

1.3
$\frac{3.22}{2.43}$
0.79

2.0
2.36
1.57

$\frac{2.36}{1.57}$

0.79
0.00

4Q99

TOTALMcf

IOTAL MC

0

$1,375,842$

832,692

$3,285,169$

$\frac{2.23}{2.23}$

2.23

0.00

0.00

31.6

$\frac{2.23}{2.23}$

0.00

0.00
Jan. Feb. 1999

$\begin{array}{lll}0.00 & 0.00 & 0.00\end{array}$

(1) ECT Canada purchases this supply from Poco Marketing, Ltd. The gas is marketed to various New York customers. This contract was terminated.

(2) Supply is delivered to Leldy. Pennsylvania for future deliveries to New York Slate.

3 U. Under this supply contracl. about 25 MMct/day will be delivered to Manhattan. illinois with the remaining 15 MMclday will be delivered to Ventura, lowa.
4) Primarily for markets in Michigan. 


\section{VOLUME AND PRICE REPORT}

\section{Long-Term Imports}

Volumes (MMct/d) \& Prices (\$MMBTU) of Natural

Gas Imported During the Past 12 Months 01/01/99 - 12/31/99

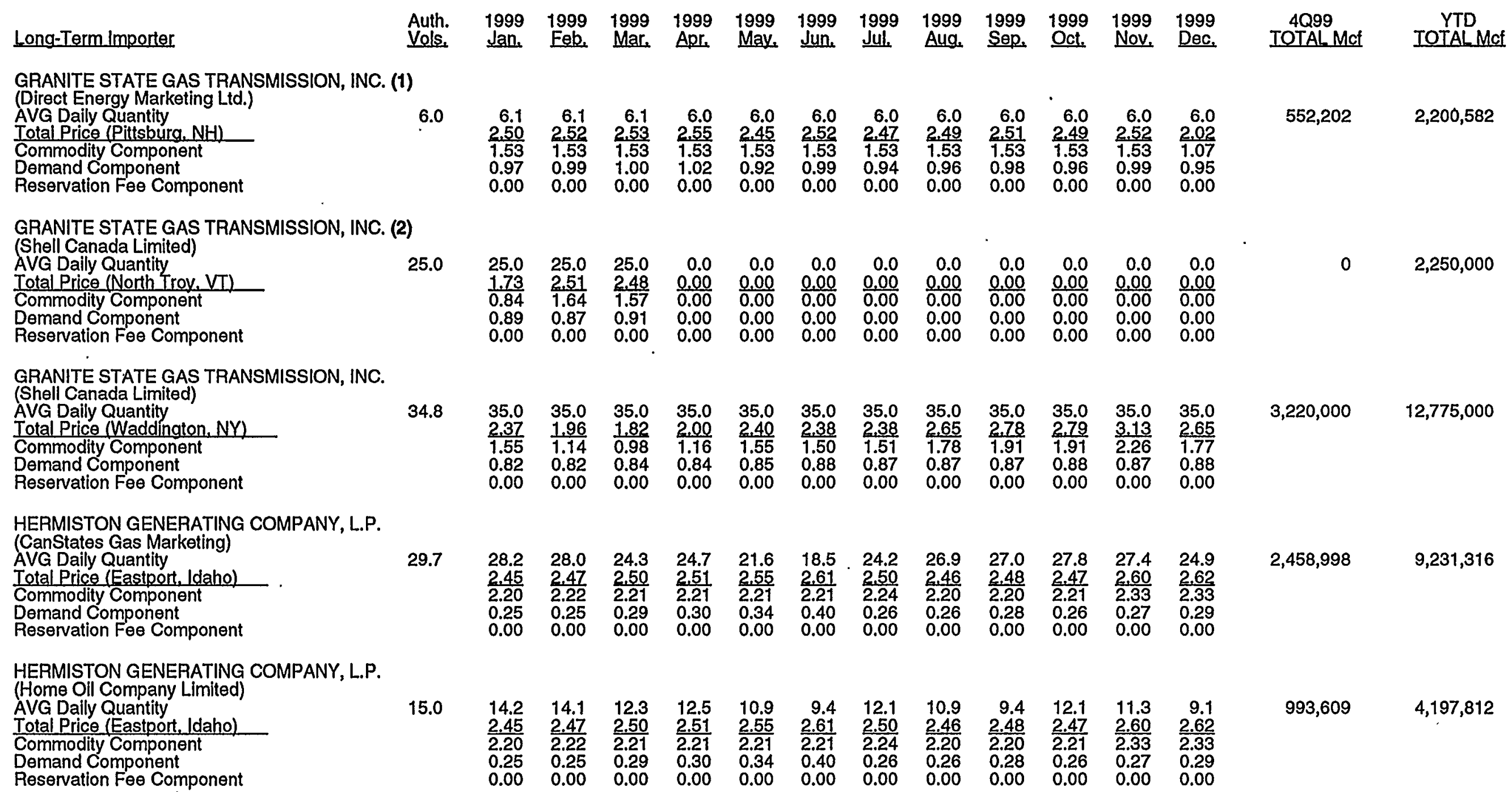

(1) As of $4 / 1 / 99$, Ihis authorizalion began imporling volumes at Pittsburg, NH.
(2) This import authorizalion expired on $3 / 31 / 99$. 
VOLUME AND PRICE REPORT

\section{Long-Term Imports}

Volumes (MMcf/d) \& Prices (\$MMBTU) of Natural

Gas Imported During the Past 12 Months 01/01/99 - 12/31/99
Long-Term Importer

HUSKY GAS MAAKETING INC. (1)

(Husky Oil Operations Ltd.)

AVG Daily Quantity

Total Price (Eastport. Idaho)

Commodity Component

Demand Component

Reservation Fee Component

IGI RESOURCES, INC.

(Mobil Oil Canada)

Total Price (Sumas, Washington)

Demand Component

Reservation Fee Component

LOCKPORT ENERGY ASSOCIATES, L.P.

(ProGas Limited)

AVG Daily Quantity

Total Price (Niagara Falls, NY)

Demand Component

MIDLAND COGENERATION VENTURE1

(Union Pacific Resources)

AVG Daily Quantity

Total Price (Noyes, Minnesota)

Commodity Component

Demand Component

Reservation Fee Component

\section{MIDLAND COGENERATION VENTURE2}

\section{(Coral Energy)}

AVG Daily Quantity

Total Price (Noyes, Minnesota)

Commodity Component

Demand Component

Reservation Fee Component
Auth

Auth. 19991999

Jan.

Eeb.

21.9

\begin{tabular}{ll}
21.3 & 21.1 \\
1.40 & 1.25 \\
\hline 1.40 & 1.25 \\
0.00 & 0.00 \\
0.00 & 0.00
\end{tabular}

$0.00 \quad 0.00$

10.0

1999

1999

1999

1999

1999

19991999

Nov Dec

TOTAL M

$\frac{2.19}{2.19} \quad 4.00$

1.42

$\frac{2.02}{2.02}$

18.7
1.84

1.84

\begin{tabular}{lr}
17.7 & 18.7 \\
1.87 & 2.09 \\
\hline 1.87 & -2.09 \\
0.00 & 0.00 \\
0.00 & 0.00
\end{tabular}

$\frac{2.47}{2.47}$

0.00

0.00

$0.00 \quad 0.00$

21.8

$\frac{3.09}{3.09}$

0.00
0.00
Commodity Component

Commodity Componen

Reservation Fee Component

\begin{tabular}{r}
0.0 \\
0.00 \\
\hline 0.00 \\
0.00
\end{tabular}

0.00
0.00
0.00

0.00
0.00

\begin{tabular}{rr}
0.0 & 0.0 \\
0.00 & 0.00 \\
\hline 0.00 & 0.00 \\
0.00 & 0.00 \\
0.00 & 0.00
\end{tabular}

0.00

12.0

\begin{tabular}{rrr}
9.3 & 3.2 & 7.7 \\
$\frac{4.06}{2.81}$ & $\frac{7.59}{2.80}$ & $\frac{4.28}{2.77}$ \\
\hline 2.81 & 3.51 & 1.28 \\
0.19 & 1.28 & 0.23
\end{tabular}

3.0

$\frac{6.88}{2.79}$

0.23

0.62

$\frac{4.20}{2.78}$

1.21

10.0

\begin{tabular}{ll}
10.0 & 10.0 \\
1.71 & 1.75 \\
\hline 1.29 & 1.26 \\
0.42 & 0.49 \\
0.00 & 0.00
\end{tabular}

10.0
1.70
1.27
0.43
0.00

10.0
1.69
1.24
0.45
0.00

\begin{tabular}{l}
10.0 \\
1.68 \\
\hline 1.23 \\
0.45 \\
0.00
\end{tabular}

10.0
$\frac{1.68}{1.21}$
0.47
0.00

10.0
$\frac{1.66}{1.21}$
0.45

\begin{tabular}{l}
1.66 \\
\hline 1.21 \\
0.45
\end{tabular}

10.0
1.67
1.20
0.47
0.00

$\begin{array}{lll}10.0 & 10.0 & 10.0 \\ \frac{1.64}{1.19} & \frac{1.66}{1.19} & \frac{1.64}{1.18} \\ 0.45 & 0.47 & 0.46 \\ 0.00 & 0.00 & 0.00\end{array}$

15.0

$\begin{array}{llll}15.0 & 15.0 & 15.0 & 15.0 \\ \frac{1.64}{1.25} & \frac{1.68}{1.23} & \frac{1.64}{1.24} & \frac{1.64}{1.22} \\ 0.39 & 0.45 & 0.40 & 0.42 \\ 0.00 & 0.00 & 0.00 & 0.00\end{array}$

\begin{tabular}{l}
15.0 \\
1.62 \\
\hline 1.21 \\
0.41 \\
0.00
\end{tabular}

\begin{tabular}{l}
15.0 \\
1.63 \\
\hline 1.20 \\
0.43 \\
0.00
\end{tabular}

\begin{tabular}{l}
15.0 \\
1.58 \\
\hline 1.17 \\
0.41 \\
0.00
\end{tabular}

\begin{tabular}{l}
15.0 \\
1.58 \\
\hline 1.17 \\
0.41 \\
0.00
\end{tabular}

\begin{tabular}{l}
15.0 \\
1.59 \\
\hline 1.16 \\
0.43 \\
0.00
\end{tabular}

15.0
1.57
1.15
0.4
0.00

0.0

$\frac{0.00}{0.00}$

0.00

0.0

0.0

$\frac{0.00}{0.00}$

0.00

0.00

(1) These volumes are delivered to San Dlego Gas \& Electric Company. 
VOLUME AND PRICE REPORT

\section{Long-Term Imports}

Volumes (MMcf/d) \& Prices (\$MMBTU) of Natural

Gas Imported During the Past 12 Months 01/01/99 - 12/31/99
Long-Term Importer

MIDLAND COGENERATION VENTURE3 (Husky Oil)

AVG Daily Quantity

Total Price (Noyes, Minnesota)

Commodity Component

Demand Component

Reservation $\mathrm{Fee}$ Component

MIDLAND COGENERATION VENTURE4

(TransCanada Pipelines Limited)

AVG Daily Quantity

Total Price (Noyes. Minnesota)

Commodity Componen

Demand Component

Reservation Fee Component

MIDLAND COGENERATION VENTURE5 (Poco Petroleums Ltd.)

AVG Daily Quantity

Total Price (Noves. Minnesota)

Demand Component

Reservation Fee Component

MIDLAND COGENERATION VENTURE 6 (Union Pacific Pesources)

AVG Daily Quantity

Total Price (Noyes, Minnesota)

Commodity Componen

Demand Component

Reservation Fee Component

MINNEGASCO, INC.

(TransCanada Pipelines Limited)

AVG Daily Quantity

Total Price (Noyes Minnesota)

Commodity Componen

Reservation Fee Component
Auth

Yuth

15.0

\begin{tabular}{ll}
15.0 & 15.0 \\
1.90 & 1.90 \\
\hline 1.51 & 1.47 \\
0.39 & 0.43
\end{tabular}

$\frac{15.0}{1.51}$

15.0
1.90
1.49
0.41

15.0
1.90

$\frac{1.90}{1.49}$

0.00

0.00

$0.00 \quad 0.00$

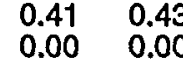

15.0

$\begin{array}{ll}15.0 & 15.0 \\ 1.63 & 1.67 \\ 1.22 & 1.21 \\ 0.41 & 0.46 \\ 0.00 & 0.00\end{array}$

$\begin{array}{ll}15.0 & 15 . \\ 1.62 & 1.60 \\ 1.21 & 1.16 \\ 0.41 & 0.44 \\ 0.00 & 0.00\end{array}$

15.0
$\frac{1.60}{1.16}$
0.44
0.00

15.0
1.59
1.16
0.43
0.00

$\begin{array}{ll}15.0 & 15.0 \\ 159 & 157\end{array}$

$\frac{1.59}{1.14}$

0.45

0.45
0.00

15.0

$\begin{array}{lll}15.0 & 15.0 & 15.0 \\ 2.42 & 2.47 & 2.44 \\ 2.04 & 2.03 & 2.05 \\ 0.38 & 0.44 & 0.39 \\ 0.00 & 0.00 & 0.00\end{array}$

$\begin{array}{lr}15.0 & 7.5 \\ 2.46 & 2.47 \\ 2.05 & 2.07 \\ 0.41 & 0.40 \\ 0.00 & 0.00\end{array}$

15.0
2.49
2.06
0.43
0.00

15.0
2.48

$\frac{2.48}{2.07}$

0.00

$0.00 \quad 0.00$

10.0

\begin{tabular}{ll}
10.0 & 10.0 \\
2.88 & 2.93 \\
\hline 2.52 & 2.52 \\
0.36 & 0.41 \\
0.00 & 0.00
\end{tabular}

10.0
2.90
2.53
0.37
0.00

10.0
2.92
2.53
0.39

0.5
2.92
2.54
0.38

0.0
0.00
0.00
0.00
0.00

0.0

$\frac{0.00}{0.00}$

0.00

0.00

50.0

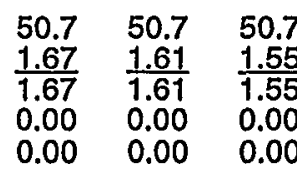

\begin{tabular}{l}
30.9 \\
1.62 \\
\hline 1.62 \\
0.00 \\
0.00
\end{tabular}

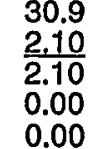

30.9
1.97
1.97
0.00
0.00
30.9
2.04
2.04
0.00

0.00 \begin{tabular}{l}
30.9 \\
2.34 \\
\hline 2.34 \\
0.00 \\
0.00
\end{tabular}
30.9
2.73
2.73
0.00

0.00
$4 Q 99$

TOTALMCf

TOTALMCf

$1,380,000$

$5,475,000$

$$
5,475,000
$$

1.49

.41

0.00

$1,380,000$

$5,475,000$

$\frac{1.56}{1.13}$

15.0
1.58
1.15

\begin{tabular}{l}
15.0 \\
1.56 \\
\hline 1.45
\end{tabular}

$15.0 \quad 15.0$

$\frac{1.57}{1.14} \frac{1.55}{1.14}$

1.55
1.14
0.41

$1,380,000$

$0.00 \quad 0.00$

$1,380,000$

$5,242,500$

610,000

$1,825,000$

$\begin{array}{lllll}0.00 & \frac{0.00}{0.00} & \frac{0.00}{0.00} & \frac{2.99}{2.59} & \frac{2.98}{2.59}\end{array}$

$\begin{array}{lllll}0.00 & 0.00 & 0.00 & 0.40\end{array}$

$\begin{array}{llll}0.00 & 0.00 & 0.00 & 0.00\end{array}$

0.39
0.00

30.9
2.38
2.38
0.00
0.00

30.9

30.9

$\frac{1.97}{1.97}$

$\frac{2.84}{2.84} \quad \frac{1.97}{1.97}$

$\begin{array}{lll}0.00 & 0.00 & 0.00\end{array}$

$2,843,904$

$13,062,082$ 


\section{VOLUME AND PRICE REPORT}

\section{Long-Term Imports}

Volumes (MMcf/d) \& Prices (\$MMBTU) of Natura

Gas Imported During the Past 12 Months 01/01/99 - 12/31/99

Long-Term Importer

N.Y. STATE ELECTRIC \& GAS CO.

(Alberta NE Gas (AEC Oil \& Gas Co.))

AVG Daily Quantity

Total Price (Waddington. NY)

Commodity Component

Demand Component

Reservation Fee Component

N.Y. STATE ELECTRIC \& GAS CO.

(Alberta NE Gas (TCGS Ltd. 2))

AVG Daily Quantity

Total Price (Waddington, NY)

Commodity Component

Demand Component

Reservation $\mathrm{Fee}$ Component

N.Y. STATE ELECTAIC \& GAS CO.

(Alberta Northeast Gas (ProGas))

AVG Daily Quantity

Total Price (Waddíngton. NY)

Commodity Component

Demand Component

Reservation Fee Component

N.Y. STATE ELECTRIC \& GAS CO.

(Alberta Northeast Gas (Producers Marketing))

AVG Daily Quantity

Total Price (Waddington. NY)

Commodity Component

Demand Component

Reservation Fee Component

N.Y. STATE ELECTRIC \& GAS CO.

(Crestar Energy)

AVG Daily Quantity

Total Price (Grand Island, NY)

Commodity Component

Demand Component

Reservation Fee Component $\begin{array}{lllllllllllll}\text { Auth. } & 1999 & 1999 & 1999 & 1999 & 1999 & 1999 & 1999 & 1999 & 1999 & 1999 & 1999 & 1999\end{array}$

Vols. Jan. Feb. Mar. Apr. Mav Jun. Jul. Aug Sep Qct. Nov Dec.

1.3

\begin{tabular}{rr}
1.3 & 1.3 \\
1.90 & $\frac{1.95}{1.12}$ \\
\hline 1.09 & 1.12 \\
0.81 & 0.83
\end{tabular}

1.3
1.80

$\frac{1.80}{0.98}$

1.3
2.03
1.19
0.84

1.3
2.52
1.67
0.85
0.00

1.3
2.47
1.59
0.88
0.00

1.3
2.48
1.61
0.87
0.00

1.3
2.82
1.95
0.87
0.00

1.3
$\frac{3.11}{2.23}$
0.88

\begin{tabular}{rrr}
1.3 & 0.6 & 1.3 \\
2.94 & $\frac{3.34}{2.34}$ & 2.67 \\
\hline 2.06 & $\frac{2.66}{1.79}$ \\
0.88 & 0.88 & 0.88 \\
0.00 & 0.00 & 0.00
\end{tabular}

6.8

\begin{tabular}{rrr}
6.8 & 6.8 & 6.8 \\
1.99 & $\frac{1.93}{1.22}$ & 1.88 \\
\hline 1.26 & 1.15 \\
0.73 & 0.71 & 0.73 \\
0.00 & 0.00 & 0.00
\end{tabular}

6.8
2.04
1.29
0.75
0.00

6.8
2.51
1.76
0.75

6.8
2.39
1.60
0.79

\begin{tabular}{r}
6.8 \\
2.46 \\
\hline 1.68 \\
0.78
\end{tabular}

6.8
2.76
1.98
0.78

0.00

0.00

0.00

5.9

\begin{tabular}{rr}
5.9 & 5.9 \\
1.92 & 1.96 \\
\hline 1.06 & 1.08 \\
0.86 & 0.88 \\
0.00 & 0.00
\end{tabular}

5.9
1.80
0.93
0.87
0.00

5.9
2.90
1.25
0.85
0.00

5.9
2.53
1.63
0.90
0.00

5.9
2.45
1.52
0.93
0.00

5.9
2.51
1.60
0.91
0.00

5.9
2.88
1.97
0.91
0.00

5.9
$\frac{3.16}{2.24}$
0.92
0.00

$\frac{2.99}{2.07}$

2.07
0.92

$\frac{3.38}{2.48}$

0.90
0.00

2.79

1.87

0.92
0.00

3.0

\begin{tabular}{rrr}
3.0 & 3.0 & 2.9 \\
1.92 & $\frac{1.96}{1.11}$ & $\frac{1.80}{0.96}$ \\
\hline 1.08 & 1.11 & 0.84 \\
0.84 & 0.85 & 0.84 \\
0.00 & 0.00 & 0.00
\end{tabular}

3.0
2.10
1.25
0.85
0.00

3.0
2.53
1.66
0.87
0.00

3.0
2.45
1.55
0.90
0.00

3.0
2.51
1.63
0.88

3.0
2.88
2.00
0.88

3.0
$\frac{3.16}{2.27}$

3.0
2.99
2.10

$0.89 \quad 0.89$

0.00

10.0

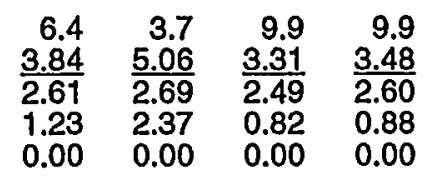

\begin{tabular}{r}
9.8 \\
3.57 \\
\hline 2.73 \\
0.84 \\
0.00
\end{tabular}

\begin{tabular}{r}
9.7 \\
3.33 \\
\hline 2.42 \\
0.91 \\
0.00
\end{tabular}

\begin{tabular}{r}
1.6 \\
7.68 \\
\hline 2.56 \\
5.12 \\
0.00
\end{tabular}

\begin{tabular}{r}
8.0 \\
3.72 \\
\hline 2.66 \\
1.06 \\
0.00
\end{tabular}

\begin{tabular}{r}
1.6 \\
8.36 \\
\hline 2.95 \\
5.41 \\
0.00
\end{tabular}

$\begin{array}{r}9.6 \\ 3.68 \\ \hline 278\end{array}$

$\frac{3.68}{2.78}$

0.90
0.00
4Q99

TOTAL MA

100,776

$\cdot 462,503$

431,275

$2,052,895$

248,003

$1,059,726$

732,097

$2,580,915$ 


\section{VOLUME AND PRICE REPORT}

\section{Long-Term Imports}

Volumes (MMcf/d) \& Prices (\$MMBTU) of Natural Gas Imported During the Past 12 Months 01/01/99 - 12/31/99

Long-Term Importer

N.Y. STATE ELECTRIC \& GAS CO. (1)

(Progas Limited)

AVG Daily Quantity

Total Price (Niagara Falls. NY)

Commodity Component

Demand Component

Reservation Fee Component

NATIONAL FUEL GAS DISTRIBUTION CORP.

(Alberta NE Gas (TCGS Ltd.))

AVG Daily Quantity

Total Price (Niagara Falls. NY)

Commodity Component

Demand Component

Reservation Fee Component

\section{NATIONAL STEEL CORPORATION}

(Direct Energy Marketing, Ltd.)

AVG Daily Quantity

Total Price (Detroit, Michigan)

Commodity Component

Demand Component

Reservation $\mathrm{Fee}$ Component

NEW JERSEY NATURAL GAS COMPANY

(Alberta NE Gas (AEC Oil \& Gas Company))

AVG Daily Quantity

Total Price (Waddington. NY)

Demand Component

Reservation Fee Component

NEW JERSEY NATURAL GAS COMPANY

(Alberta NE Gas (TCGS Ltd. 1))

AVG Daily Quantity

Total Price (Waddington, NY)

Commodity Componen

Demand Component

Reservation Fee Component
Auth. 199

Vols. 199919991999

9.0

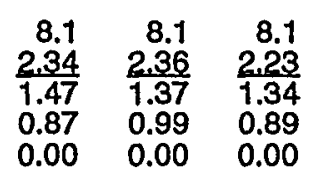

8.1
2.44
1.51
0.93
0.00

8.1
2.52
1.61
0.91
0.00

8.9
2.41
1.55
0.86
0.00

8.1
2.43
1.50
0.93

0.93
0.00

2.90
1.97
0.93

0.93

$\frac{3.04}{2.07}$

0.97
0.00

$\frac{3.17}{2.23}$

0.94
0.00

$\frac{3.27}{2.19}$

2.19
1.08
0.00

$0.00 \quad 0.0$

10.0

$\begin{array}{lll}10.0 & 10.0 & 10.0 \\ 2.00 & \frac{1.93}{1.24} & \frac{1.88}{1.16} \\ 1.28 & 1.69 & 0.72 \\ 0.72 & 0.69 & 0.7 \\ 0.00 & 0.00 & 0.00\end{array}$

10.0
2.03
1.30
0.73
0.00

10.0
2.51
1.77
0.74
0.00

10.0
2.38
1.61
0.77
0.00

10.0
2.45
1.69
0.76
0.00

10.0
2.76
2.00
0.76
0.00

10.0
3.15
2.38
0.77
0.00

10.0

$\frac{2.79}{2.02}$

7.3

$\frac{3.22}{2.45}$

0.77

0.00

18.0

\begin{tabular}{lrr}
25.9 & 26.4 & 17.1 \\
2.60 & - & 2.55 \\
\hline 2.60 & 2.55 & 2.55 \\
0.00 & 0.00 & 0.55 \\
0.00 & 0.00 & 0.00
\end{tabular}

22.2
2.35
2.35
0.00
0.00

16.3
2.38
2.38
0.00

16.2
2.41
2.41
0.00

17.6
2.27
2.27

$0.00 \quad 0.00$

$\begin{array}{lll}0.00 & 0.00 & 0.00\end{array}$
20.5
2.42

$\frac{2.42}{2.42}$

0.00

0.00 $\begin{array}{r}13.1 \\ 2.47 \\ \hline\end{array}$

2.47

0.00

0.00 $\begin{array}{r}22.5 \\ 2.43 \\ \hline 2.43\end{array}$

2.43

0.00 \begin{tabular}{ll}
21.5 & 25.2 \\
2.47 & 2.62 \\
\hline 2.47 & 2.62 \\
0.00 & 0.00
\end{tabular}

$\begin{array}{ll}0.00 & 0.00 \\ 0.00 & 0.00\end{array}$
1.9

\begin{tabular}{rrr}
1.9 & 1.9 & 1.9 \\
1.90 & 1.95 & 1.80 \\
\hline 1.09 & 1.12 & 0.98 \\
0.81 & 0.83 & 0.82
\end{tabular}

$\begin{array}{lll}0.81 & 0.83 & 0.82 \\ 0.00 & 0.00 & 0.00\end{array}$

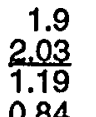

1.9
2.52
1.67
0.85

1.9
2.47
1.59
0.88

1.9
2.48
1.61

2.48
1.61
0.87

0.87
0.00
1.9
2.82
1.95
0.87

0.00

\begin{tabular}{|c|c|}
\hline $\begin{array}{l}25.2 \\
1.99 \\
1.26 \\
0.73\end{array}$ & $\begin{array}{l}25.2 \\
1.93 \\
1.22 \\
0.71 \\
0.00\end{array}$ \\
\hline
\end{tabular}

25.2
2.04
1.29
0.75
0.00

25.2
2.51
1.76
0.75
0.00

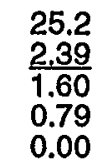

25.2
2.46
1.68
0.78
0.00

\begin{tabular}{l}
25.2 \\
2.76 \\
\hline 1.98 \\
0.78 \\
0.00
\end{tabular}

$\begin{array}{r}1.9 \\ 3.11 \\ \hline 2.93\end{array}$

$\frac{3.11}{2.23}$

1.9
2.94
2.06

2.06
0.88

$\begin{array}{r}1.2 \\ 3.34 \\ \hline\end{array}$

$\frac{3.34}{2.46}$

0.88

0.00

1.9
1.67

$\frac{2.67}{1.79}$

0.88
0.00

157,626

688,884

(1) NYSEG's imports include 1st Qtr 1999: 433,215 at Champlain, NY, 256,294 at Nlagara, NY, and 40,228 at Grand Island, NY; 2nd QIr 1999: 311,893 at Champlain, NY, and 22,923 at Waddington. NY, and 426,855 at Nlagara, NY: 3rd Oir 1999: 260,762 at Champlain, NY, 346,713 at Waddinglon, NY, and 139,416 al Nlagara, NY; 4th Qir 1999: 424,917 at Champlain, NY, 170,167 al Waddinglon, NY, 108,395 at Nlagara, NY, and 13,045 at Grand Island, NY. 


\section{VOLUME AND PRICE REPORT}

\section{Long-Term Imports}

Volumes (MMcf/d) \& Prices (\$MMBTU) of Natural

Gas Imported During the Past 12 Months 01/01/99 - 12/31/99

Long-Term Importer

NEW JERSEY NATURAL GAS COMPANY

(Alberta NE Gas (TCGS Ltd. 2))

AVG Daily Quantity

Total Price (Waddington. NY)

Commodity Component

Demand Component

Reservation Fee Component

NEW JERSEY NATURAL GAS COMPANY

(Alberta Northeast Gas (ProGas))

AVG Daily Quantity

Total Price (Waddington, NY)

Commodity Component

Demand Component

Reservation Fee Component

\section{NEW JERSEY NATURAL GAS COMPANY}

(Alberta Northeast Gas (Producers Mktg.))

AVG Daily Quantity

Total Price (Waddington. NY)

Commodity Component

Demand Component

Reservation Fee Component

NIAGARA MOHAWK POWER CORPORATION

(TCGS Ltd.)

AVG Daily Quantity

Total Price (Waddington. NY)

Commodity Component

Demand Component

Reservation Fee Component

NORTH JERSEY ENERGY ASSOCIATES

(ProGas Limited)

AVG Daily Quantity

Total Price (Niagara Falls, NY)

Commodity Component

Demand Component

Reservation Fee Component
Vols.

Jan. Feb. 19991999

19991999

1999

5.3

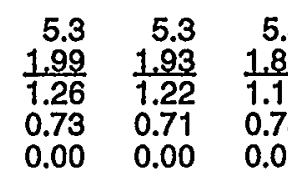

4.6

\begin{tabular}{rr}
4.6 & 4.6 \\
1.92 & $\frac{1.96}{1.08}$ \\
\hline 1.06 & 0.86 \\
0.88
\end{tabular}

$0.00 \quad 0.00$

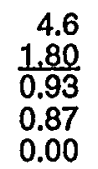

4.6
2.10

$\frac{2.10}{1.25}$

$.87 \quad 0.85$

3.0

\begin{tabular}{rr}
3.0 & 3.0 \\
1.92 & $\frac{1.96}{1.11}$ \\
\hline 1.08 & 1.11 \\
0.84 & 0.85 \\
0.00 & 0.00
\end{tabular}

2.9

$\frac{1.80}{0.96}$

0.96
0.84

0.00

3.0
2.10
1.25
0.85
0.00

3.0

$\frac{2.53}{1.66}$

0.87
0.00

$\begin{array}{r}3.0 \\ 2.45 \\ \hline 1.55\end{array}$

1.55
0.90

0.00

3.0
2.51
1.63
0.88

0.88
0.00

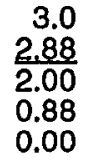

$\begin{array}{r}3.0 \\ 3.16 \\ \hline .27\end{array}$

0.89
0.00

3.0
2.99
2.10
0.89
0.00

$\frac{3.37}{2.44}$

0.93
0.00

3.0
2.79
1.90

0.89

0.89
0.00

51.0

$\begin{array}{lll}51.0 & 51.0 & 51.0 \\ \frac{2.16}{1.35} & \frac{2.33}{1.41} & \frac{2.0}{1.20} \\ 0.81 & 0.92 & 0.8 \\ 0.00 & 0.00 & 0.00\end{array}$

$51.0 \quad 51.0$

$\frac{2.33}{1.47} \frac{2.80}{1.96}$

$\begin{array}{lll}51.0 & 51.0 & 51.0\end{array}$

$\frac{2.68}{1.78}$

51.0
2.70
1.84
0.86

0.86

0.84
0.00

0.90
0.00

0.86
0.00

$\frac{3.06}{2.20}$

\begin{tabular}{l}
51.0 \\
3.39 \\
\hline
\end{tabular}

$\frac{3.39}{2.51}$

51.0

$\frac{2.97}{2.12}$

2.12
0.85
0.00

51.6

$\frac{3.48}{2.59}$

2.59
0.89
0.00

51.6

$\frac{2.48}{1.63}$

1.63
0.85

-

24.0

$\begin{array}{llll}22.4 & 22.4 & 20.9 & 21.3 \\ \frac{3.30}{2.46} & \frac{3.39}{2.45} & \frac{3.00}{2.11} & \frac{3.05}{2.13} \\ 0.84 & 0.94 & 0.89 & 0.92 \\ 0.00 & 0.00 & 0.00 & 0.00\end{array}$

21.6
2.97
2.08
0.89
0.00

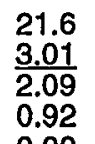

21.6
2.96
2.05
0.91
0.00

\begin{tabular}{l}
21.6 \\
2.96 \\
\hline 2.05 \\
0.91 \\
0.00
\end{tabular}

\begin{tabular}{l}
21.6 \\
2.99 \\
\hline 2.04 \\
0.95 \\
0.00
\end{tabular}

\begin{tabular}{l}
21.6 \\
2.96 \\
\hline 2.04 \\
0.92 \\
0.00
\end{tabular}

21.6
2.98
2.03
0.95

21.6
2.96

$\frac{2.96}{2.04}$

0.92

0.00

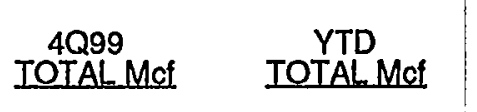

$425,250 \quad 1,858,500$

$343,136 \quad 1,604,396$

$241,704 \quad 1,053,596$

$4,728,356$

$18,651,356$

$1,991,340$

$7,910,292$ 
VOLUME AND PRICE REPORT

Long-Term Imports

Volumes (MMcf/d) \& Prices (\$MMBTU) of Natural

Gas Imported During the Past 12 Months 01/01/99 - 12/31/99

\section{Long-Term Importer}

NORTHEAST ENERGY ASSOCIATES

(ProGas Limited)

AVG Daily Quantity

Total Price (Niagara Falls, NY)

Commodity Component

Demand Component

Reservation Fee Component

\section{NORTHERN MINNESOTA UTILITIES}

(TCGS Ltd.)

AVG Daily Quantity

Total Price (Noyes. Minnesota)

Demand Component

Reservation Fee Component

NORTHERN MINNESOTA UTILITIES - EASTERN MARKET (1)

(TCGS Ltd.)

AVG Daily Quantity

\begin{tabular}{ll}
18.3 & 17.6 \\
2.02 & 1.92 \\
\hline 1.80 & 1.69 \\
0.22 & 0.23
\end{tabular}

Auth. $\quad 1999 \quad 1999$

1999

1999

1999

1999

199

ul. Au

48.0

$\begin{array}{lll}49.6 & 49.6 \quad 49.6\end{array}$

$\begin{array}{lll}\frac{3.60}{2.76} & \frac{3.67}{2.73} & \frac{3.56}{2.73}\end{array}$

$0.84 \quad 0.94$

0.00

$0.00 \quad 0.00$
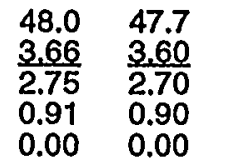

36.5
$\frac{3.91}{2.71}$
1.20

\begin{tabular}{l}
45.8 \\
3.63 \\
\hline
\end{tabular}

$\frac{3.63}{2.68}$

2.68
0.95

0.00

$\frac{3.63}{2.69}$

2.69
0.94

0.94
0.00

10.0

$\begin{array}{llll}10.0 & 10.0 & 10.0 & 10.0 \\ \frac{1.53}{1.12} & \frac{1.47}{1.00} & \frac{1.41}{1.00} & \frac{1.57}{1.13} \\ 0.41 & 0.47 & 0.41 & 0.44 \\ 0.00 & 0.00 & 0.00 & 0.00\end{array}$

10.0
2.05
1.62
0.43
0.00

$10.1 \quad 10.1$

$\frac{1.92}{1.47} \quad \frac{1.99}{1.57}$

$0.45 \quad 0.42$

10.0
2.29
1.86
0.43
0.00

Total Price (Noyes, Minnesota)
Total Price (International Falls, MN)

Commodity Component

Demand Component

Reservation Fee Component

29.8

\section{NORTHERN MINNESOTA UTILITIES - WESTERN MARKET}

(TCGS Ltd.)

AVG Daily Quantity

Total Price (Warroad, Minnesota)

Commodity Component

Demand Component

Reservation Fee Component

NORTHERN STATES POWER COMPANY

(Amoco Canada (WI))

AVG Daily Quantity

Commodity Componen

Demand Component

Reservation Fee Component

15.0

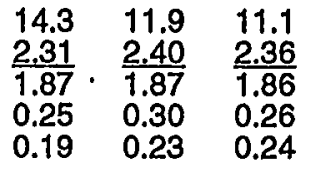

5.9
2.80
1.87
0.48
0.45

\begin{tabular}{r}
5.9 \\
2.79 \\
\hline 1.86 \\
0.48 \\
0.45
\end{tabular}

\begin{tabular}{r}
5.9 \\
2.80 \\
\hline 1.86 \\
0.49 \\
0.45
\end{tabular}

\begin{tabular}{r}
5.9 \\
2.77 \\
\hline 1.85 \\
0.47 \\
0.45
\end{tabular}

5.9
2.78
1.86
0.47
0.45

5.9
2.80
1.86
0.49

0.49
0.45

(1) Beginning in January 1999, additional volumes were imported under a short-term authorization using various suppliers. $\frac{3.62}{2.67}$
0.95

3.59

$\frac{3.59}{2.67}$

0.92
0.00

10.0
2.68
2.25
0.43

10.0
2.33
1.91
0.42
0.00

10.0
2.33
1.91
0.42
0.00

$\begin{array}{ll}10.0 & 10.0\end{array}$

$\frac{2.76}{2.33} \quad \frac{1.89}{1.48}$

$0.43 \quad 0.41$

$\begin{array}{ll}0.43 & 0.41 \\ 0.00 & 0.00\end{array}$

$\begin{array}{rrrr}0.7 & 0.8 & 2.0 & 3.2 \\ \frac{2.77}{2.77} & \frac{2.42}{2.42} & \frac{2.99}{2.99} & \frac{2.14}{2.14} \\ 0.00 & 0.00 & 0.00 & 0.00 \\ 0.00 & 0.00 & 0.00 & 0.00\end{array}$

3.2
2.14
2.14
0.00
0.00

183,861

$2,248,893$

4Q99

$4,414,899$

919,963

$3,654,569$

14,445

363,326

$\frac{2.77}{2.77} \quad \frac{2.42}{2.42} \quad \frac{2.99}{2.99} \quad \frac{2.14}{2.14}$

$\begin{array}{llll}2.77 & 2.42 & 2.99 & 2.14 \\ 0.00 & 0.00 & 0.00 & 0.00\end{array}$

$\begin{array}{llll}0.00 & 0.00 & 0.00 & 0.00 \\ 0.00 & 0.00 & 0.00 & 0.00\end{array}$

$\begin{array}{rr}5.9 & 10.3\end{array}$

$\frac{2.79}{1.87}$

2.24

11.2

$\frac{2.27}{1.89}$

842,334

$3,045,549$ 
कृष्ठ

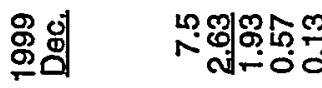

요을

क्ष.व

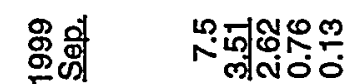

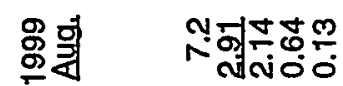

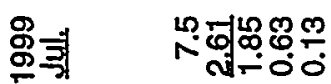

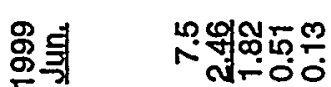

हे

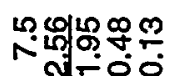

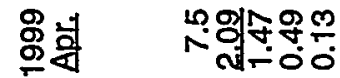

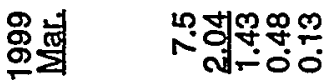

รัㅇํㅁ

수워ํㅇํㅇํำ

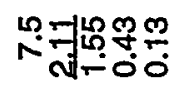

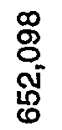

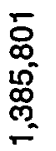

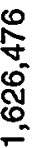

$\infty$
0
0
8
8

हैं

$\frac{1}{8}$

है

ํㅗㄹㅇำ

in

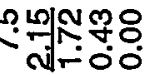
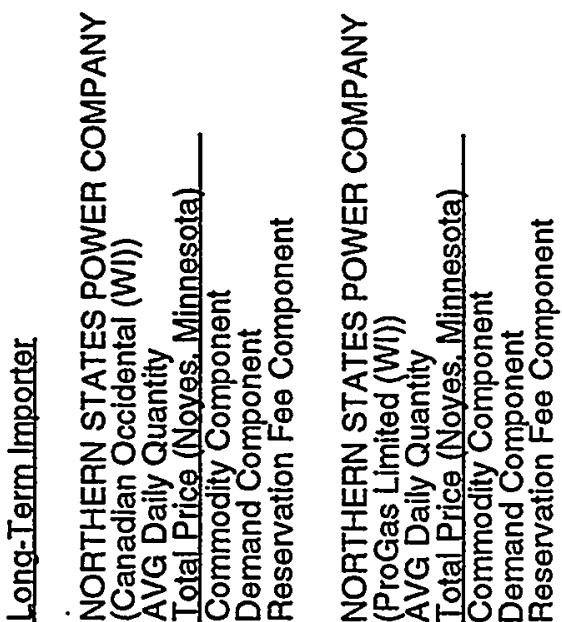

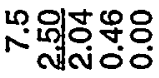

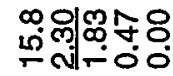

क데용

우ㅇㅟㅟ용ㅇㅇ

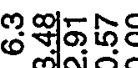

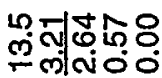

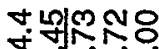

우이용ㅇㅇㅇ

ํํำ

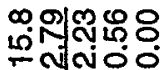

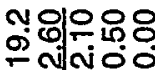

어윘요용

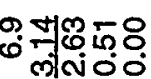

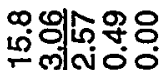

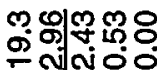

유부잉ㅇㅇㅇ

ำ ำ

的ㄴำ

뚜윰ㅇㅇㅇ

우이묘용

aido

10 जीं

- Nvio

Nivio

ำ 웡ㅇํㅇㅇㅇ

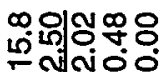

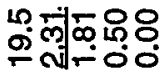

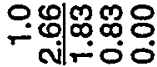

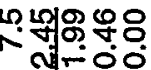

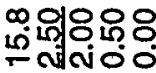

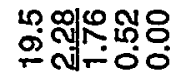

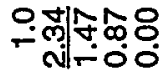

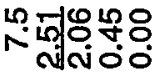

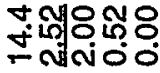

우씼용요

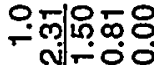

수엉웡ㅇㅇㅇ

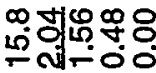

모이용ㅇㅇㅇㅇ

の

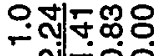

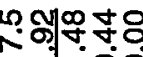

$\infty$ 인

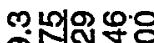

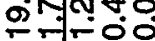

ํㅐㅁำ

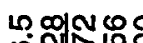

$\infty$ 이윰용

ตํํำ용

कर्षा०००

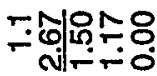

$\mathbb{O}$

N
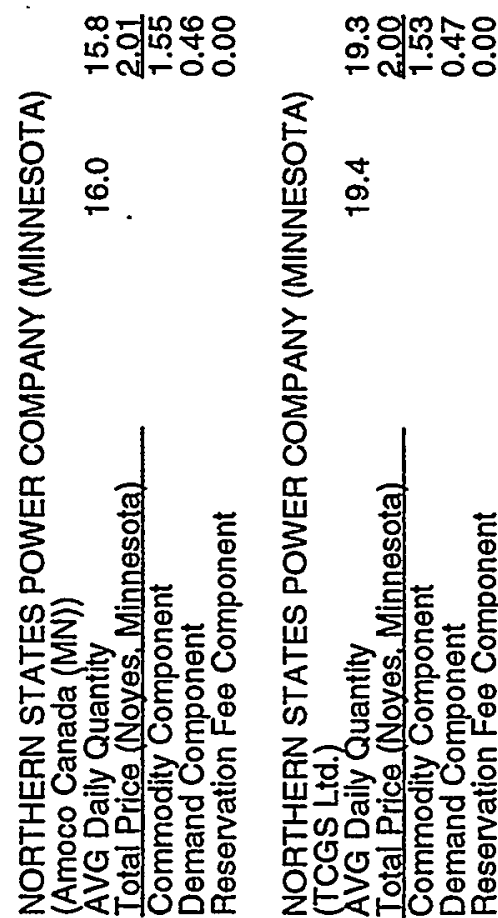

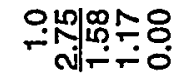

$\stackrel{0}{\circ}$ 


\section{VOLUME AND PRICE REPORT}

\section{Long-Term Imports}

Volumes (MMcf/d) \& Prices (\$MMBTU) of Natura

Gas Imported During the Past 12 Months 01/01/99 - 12/31/99

Long-Term Importer

NORTHWEST NATURAL GAS COMPANY (Amoco Canada)

AVG Daily Quantity

Total Price (Sumas, Washington)

Commodity Component

Demand Component

Reservation Fee Component

\section{NORTHWEST NATURAL GAS COMPANY} (CanWest Gas Supply)

AVG Daily Quantity

Total Price (Sumas. Washington)

Commodity Component

Demand Component

Reservation Fee Component

\section{NORTHWEST NATUAAL GAS COMPANY}

(Engage Energy Canada)

AVG Daily Quantity

Total Price (Eastport, Idaho)

Commodity Compone

Demand Component

Reservation Fee Component

NORTHWEST NATURAL GAS COMPANY

(Poco Petroleums Limited)

AVG Daily Quantity

Total Price (Eastport. Idaho)

Commodity Component

Demand Component

Reservation Fe日 Component

NORTHWEST NATURAL GAS COMPANY

(Summit Resources Limited)

AVG Daily Quantity

Total Price (Eastport, Idaho)

Commodity Component

Demand Component

Reservation $\mathrm{Fee}$ Component

\section{Auth.}

$\begin{array}{lllll}\text { Auth. } & 1999 & 1999 & 1999 & 1999\end{array}$

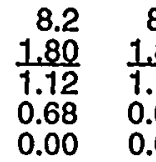

\begin{tabular}{rr}
8.2 & 8.2 \\
1.80 & $\frac{1.80}{1.12}$ \\
\hline .68 & 0.68 \\
0.00 & 0.00
\end{tabular}

8.2
1.80
1.12
0.68
0.00

8.2
1.80
0.12
0.08

8.2
1.80
0.12

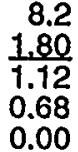
8.2
1.80
1.12
$\frac{1.80}{1.12}$
0.68

$0.00 \quad 0.00$ $\frac{1.80}{1.12}$

1.12

0.00

92.0

$\begin{array}{llllllllllll}90.9 & 93.9 & 94.7 & 94.7 & 94.7 & 98.0 & 98.1 & 87.9 & 94.7 & 94.7 & 94.6 & 94.7 \\ \frac{2.94}{2.28} & \frac{1.81}{1.17} & \frac{1.58}{0.90} & \frac{1.54}{0.91} & \frac{1.98}{1.35} & \frac{1.92}{1.31} & \frac{1.95}{1.34} & \frac{2.29}{1.61} & \frac{2.53}{1.90} & \frac{2.42}{1.79} & \frac{2.95}{2.26} & \frac{2.31}{1.62} \\ 0.66 & 0.64 & 0.68 & 0.63 & 0.63 & 0.61 & 0.61 & 0.68 & 0.63 & 0.63 & 0.69 & 0.69 \\ 0.00 & 0.00 & 0.00 & 0.00 & 0.00 & 0.00 & 0.00 & 0.00 & 0.00 & 0.00 & 0.00 & 0.00\end{array}$

23.3

$\begin{array}{lll}13.8 & 13.8 & 13.8 \\ \frac{2.10}{1.58} & \frac{2.10}{1.55} & 2.30 \\ 0.26 & 0.29 & 0.25 \\ 0.26 & 0.26 & 0.26\end{array}$

13.8
$\frac{1.95}{1.43}$
0.26
0.26

12.8
2.14
1.58
0.28
0.28

13.8
2.14
1.61
0.27
0.26

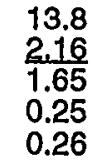

13.8

$\frac{2.14}{1.63}$

0.25

0.25
0.26

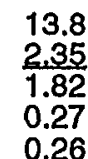

13.8
2.62
2.10
0.26
0.26

13.8

$\frac{2.69}{2.16}$

2.16

0.27
0.26

15.5

$\begin{array}{llll}15.3 & 15.2 & 15.3 & 10.8 \\ 2.09 & 2.13 & 2.11 & 2.21 \\ 1.60 & 1.60 & 1.61 & 1.59 \\ 0.24 & 0.28 & 0.25 & 0.37 \\ 0.25 & 0.25 & 0.25 & 0.25\end{array}$

8.0

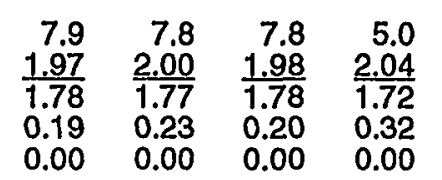

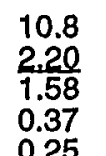

10.8
2.17
1.58
0.34
0.25

10.2
2.31
1.68
0.36
0.27

10.8
2.18
1.58
0.35
0.25

15.3
2.52
2.02
0.25
0.25

15.3
2.60
2.09
0.26
0.25

15.3
2.60
2.10
0.25
0.25

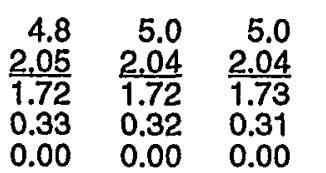

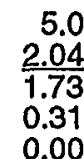

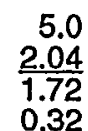

$\frac{2.04}{1.72}$ 0.32

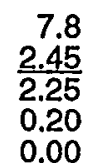

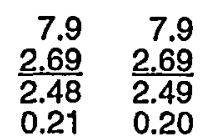

$\frac{2.69}{2.48} \quad \frac{2.69}{2.49}$

$0.21 \quad 0.20$
4Q99

TOTAL MC

YTD

TOTAL MCf

$755,780 \quad 2,998,475$

$8,708,394 \quad 34,413,803$

$1,270,217$

$5,007,597$

$1,407,763$

$4,727,279$

720,389

$2,339,497$ 
VOLUME AND PRICE REPORT

\section{Long-Term Imports}

Volumes (MMcfld) \& Prices (\$MMBTU) of Natural

Gas Imported During the Past 12 Months 01/01/99 - 12/31/99
Long-Term Importer

NUI CORPORATION (1)

(TCGS Ltd.)

AVG Daily Quantity

Total Price (Niagara Falls. NY)

Commodity Component

Demand Component

Reservation Fee Component

NW ALASKAN PIPELINE CO-Pan-Alberta Gas (I)

(Pan-Alberta Gas Ltd.)

AVG Daily Quantity.

Commodity Component

Demand Component

Reservation Fee Component

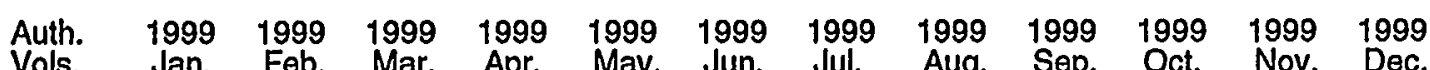

vols. Jan. Eeb. Mar. Apr. May. Jun. Jul. Aug, Sep. Oct. Nov. Dec.

2.0

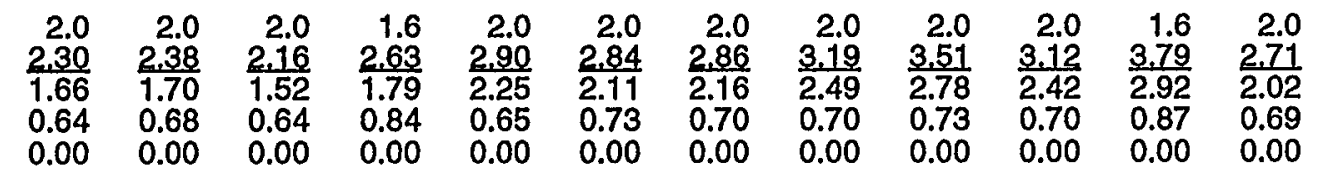

NW ALASKAN PIPELINE CO-Pan-Alberta Gas (II)

(Pan-Alberta Gas Ltd.)

AVG Daily Quantity

600.0

Total Price (Port of Morgan. MT)

Commodity Component

Demand Component

Reservation Fee Component

200.0
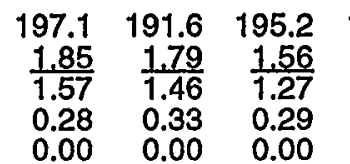

194.0
1.70
1.40
0.30
0.00

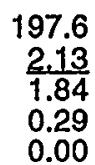

200.7
2.12
1.83
0.29
0.00

202.6
2.03
1.79
0.24
0.00

$\begin{array}{rr}198.6 & 201.8 \\ 2.31 & 2.57\end{array}$

$\frac{2.31}{2.07}$

$0.00 \quad 0.00$

\begin{tabular}{rrr}
475.1 & 473.7 & 46 \\
1.58 & 1.47 & 1 \\
\hline 1.57 & 1.46 & 1 \\
0.01 & 0.01 & 0.0 \\
0.00 & 0.00 & 0.00
\end{tabular}

$\begin{array}{ll}466.2 & 473 \\ 1.28 & 1.27 \\ 0.01 & 1.4 \\ 0.00 & 0.0\end{array}$

\begin{tabular}{rr}
473.9 & 47 \\
1.41 & 1 \\
\hline 1.40 & 1 \\
0.01 & 0.04 \\
0.00 & 0.00
\end{tabular}
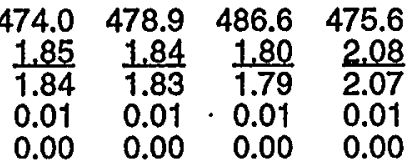

$\begin{array}{r}479.8 \\ 2.33 \\ \hline 2.32\end{array}$

$\frac{2.33}{2.32}$

OCEAN STATE POWER

(ProGas Limited)

AVG Daily Quantity

Total Price (Niagara Falls. NY)

Commodity Component

Demand Component

Reservation Fee Component

OCEAN STATE POWER II

(ProGas Limited)

AVG Daily Quantity

Total Price (Niagara Falls. NY)

Commodity Component

Demand Component

Reservation Fee Component

\section{0}

$\begin{array}{llll}50.0 & 50.0 & 50.0 & 50 \\ 2.02 & 1.94 & 1.89 & 1.81 \\ 1.17 & 1.07 & 1.01 & 0.9 \\ 0.85 & 0.87 & 0.88 & 0.8 \\ 0.00 & 0.00 & 0.00 & 0.0\end{array}$

50.0
1.85
0.98
0.87
0.00

50.0
1.83
0.96
0.87

0.96
0.87

0.0

25.0

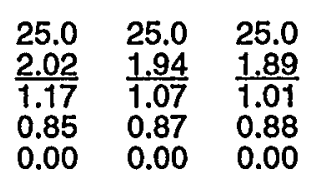

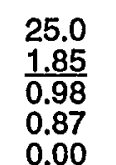

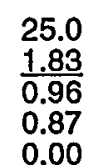

$50.0 \quad 50.0$

$\frac{1.81}{0.91} \quad \frac{1.81}{0.88}$

$0.90 \quad 0.93$

$0.00 \quad 0.00$

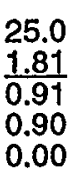

\begin{tabular}{l}
25.0 \\
1.81 \\
\hline 0.91 \\
0.90 \\
0.00
\end{tabular}

0.00

$\begin{array}{llllll}25.0 & 25.0 & 25.0 & 25.0 & 25.0 & 25.0 \\ \frac{1.81}{0.88} & \frac{1.80}{0.89} & \frac{1.80}{0.89} & \frac{1.81}{0.88} & \frac{1.80}{0.88} & \frac{1.80}{0.87} \\ 0.93 & 0.91 & 0.91 & 0.93 & 0.92 & 0.93 \\ 0.00 & 0.00 & 0.00 & 0.00 & 0.00 & 0.00\end{array}$

4099 IOTALMGI

172,562

YTD
TOTAL MC

\section{5,211}

$17,296,997$

$71,286,366$

$43,319,472$

$173,261,449$

\subsection{17}

2.16
0.01

$\begin{array}{r}457.7 \\ 2.53 \\ \hline 2.52\end{array}$

$\frac{2.53}{2.52}$
0.01

1.81

1.80

0.01
0.00

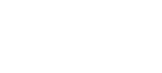

$4,600,000$

$18,250,000$

$2,300,000$

$9,125,000$

(1) Gas supplies NUl's Waverly Gas Service and Valley Cilies Gas Service LDC divisions. 


\section{VOLUME AND PRICE REPORT}

Long-Term Imports

Volumes (MMcf/d) \& Prices (\$MMBTU) of Natural

Gas Imported During the Past 12 Months 01/01/99 - 12/31/99

Long-Term Importer

OCEAN STATE POWER II

(TCGS Ltd.)

AVG Daily Quantity

Total Price (Niagara Falls. NY)

Commodity Component

Demand Component

Reservation Fee Component

ORANGE AND ROCKLAND UTILITIES, INC.

(Wascana Energy Marketing)

AVG Daily Quantity

Total Price (Niagara Falls. NY)

Commodity Componen

Demand Component

Reservation Fee Component

ORCHARD GAS CORPORATION (1)

(ProGas Limited)

AVG Daily Quantity

Total Price (Waddington. NY)

Commodity Component

Demand Component

Reservation Fee Component

PAN-ALBERTA GAS (U.S.) INC. (2)

(Pan-Alberta Gas Ltd.)

AVG Daily Quantity

Total Price (Eastport, Idaho)

Commodity Component

Demand Component

Reservation Fee Component

PAWTUCKET POWER ASSOCIATES

(Anderson Exploration Ltd.)

AVG Daily Quantity

Total Price (Waddíngton, NY)

Commodity Component

Demand Component

Reservation Fee Component

\section{Auth}

$\begin{array}{lllllllllllll}\text { Auth. } & 1999 & 1999 & 1999 & 1999 & 1999 & 1999 & 1999 & 1999 & 1999 & 1999 & 1999 & 1999\end{array}$

25.0

$\begin{array}{ll}25.0 & 25.0 \\ 2.02 & \frac{1.94}{1.12} \\ 1.20 & 1.12 \\ 0.82 & 0.82\end{array}$

$\begin{array}{ll}0.00 & 0.00\end{array}$

25.0
1.89
1.05
0.84
0.00

25.0
1.85
1.02
0.83
0.00

25.0
1.83
1.00
0.83
0.00

25.0
1.80
0.95
0.85
0.00

25.0
1.81
0.92
0.89
0.00

25.0
1.80
0.93
0.87
0.00

25.0
1.80
0.92
0.88
0.00
$25.0 \quad 25.0 \quad 25.0$
$1.81 \cdot 1.80$
0.940 .94
$0.87 \quad 0.86$
$\frac{1.81}{0.94}$
0.94
0.87

25.0

\begin{tabular}{ll}
25.3 & 25.3 \\
3.32 & $\frac{3.18}{2.06}$ \\
\hline 2.26 & 0.06 \\
0.81 & 0.89 \\
0.25 & 0.23
\end{tabular}

$\begin{array}{lll}25.3 & 25.3 & 25.3 \\ 2.69 & 2.54 & 2.86 \\ 1.70 & 1.61 & 1.90 \\ 0.80 & 0.75 & 0.74 \\ 0.19 & 0.18 & 0.22\end{array}$

24.9
2.94
1.80
0.94
0.20

24.5
2.95
1.84
0.90
0.21

\begin{tabular}{l}
24.9 \\
3.28 \\
\hline 2.21 \\
0.82 \\
0.25
\end{tabular}

\begin{tabular}{l}
25.3 \\
3.63 \\
\hline 2.49 \\
0.86 \\
0.28
\end{tabular}

25.0

$\frac{3,25}{2.18}$

24.9

24.9
3.63
2.48

$\frac{3.63}{2.48}$

$\begin{array}{ll}0.82 & 0.87 \\ 0.25 & 0.28\end{array}$

$\begin{array}{r}24.9 \\ 3.53 \\ \hline\end{array}$

$\frac{3.53}{2.44}$

-

25.0

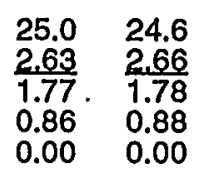

$\begin{array}{ll}24.7 & 25.0 \\ 2.62 & 2.57 \\ 1.76 & 1.69 \\ 0.86 & 0.88 \\ 0.00 & 0.00\end{array}$

24.7
2.53
1.64
0.89
0.00

25.0
2.51
1.59
0.92
0.00

25.0
$\frac{2.57}{1.66}$
0.01

$\begin{array}{ll}0.02 & 0.91 \\ 0.00\end{array}$

25.0
2.67
1.76
0.91
0.00

25.0
2.80

1.88

$0.00 \quad 0.92$
\begin{tabular}{lll}
25.0 & 25.0 & 25.0 \\
3.20 & 3.28 & 3.32 \\
\hline
\end{tabular}
$\begin{array}{lll}2.27 & 2.36 & 2.35\end{array}$
$\begin{array}{lll}0.93 & 0.92 & 0.97\end{array}$
$0.00 \quad 0.97$

240

\begin{tabular}{|c|c|c|c|c|c|c|c|c|c|c|c|}
\hline $\begin{array}{r}235.9 \\
1.75 \\
1.75 \\
0.00 \\
0.00\end{array}$ & $\begin{array}{r}229.3 \\
1.63 \\
1.63 \\
0.00 \\
0.00\end{array}$ & $\begin{array}{r}187.2 \\
1.51 \\
1.51 \\
0.00 \\
0.00\end{array}$ & $\begin{array}{r}192.5 \\
1.08 \\
1.08 \\
0.00 \\
0.00\end{array}$ & $\begin{array}{r}203.3 \\
1.47 \\
1.47 \\
0.00 \\
0.00\end{array}$ & $\begin{array}{r}161.1 \\
1.14 \\
1.14 \\
0.00 \\
0.00\end{array}$ & $\begin{array}{r}219.4 \\
1.46 \\
1.46 \\
0.00 \\
0.00\end{array}$ & $\begin{array}{r}218.7 \\
1.70 \\
1.70 \\
0.00 \\
0.00\end{array}$ & $\begin{array}{r}209.3 \\
2.04 \\
2.04 \\
0.00 \\
0.00\end{array}$ & $\begin{array}{r}242.5 \\
1.87 \\
1.87 \\
0.00 \\
0.00\end{array}$ & $\begin{array}{r}232.6 \\
2.38 \\
2.38 \\
0.00 \\
0.00\end{array}$ & $\begin{array}{r}237.6 \\
1.68 \\
1.68 \\
0.00 \\
0.00\end{array}$ \\
\hline
\end{tabular}

8.2

$\begin{array}{rrrr}6.6 & 6.7 & 2.7 & 7.1 \\ \frac{2.43}{1.51} & \frac{2.36}{1.34} & \frac{3.64}{1.43} & \frac{2.38}{1.48} \\ 0.92 & 1.02 & 2.21 & 0.90 \\ 0.00 & 0.00 & 0.00 & 0.00\end{array}$

$\begin{array}{ll}7.4 & 7.1 \\ \frac{2.59}{1.74} & 2.59 \\ 0.85 & 0.95 \\ 0.00 & 0.00\end{array}$

6.9
2.68
1.72

2.68
1.72
0.96

6.2
$\frac{2.87}{1.90}$
0.97
0.00

$\frac{7.2}{3.30}$
2.11
1.19
0.00

6.7
2.95
1.98

$\frac{2.95}{1.98}$

6.7

$\frac{3.16}{2.16}$

7.2
2.70
1.80

1.80

0.00

4Q99

TOTAL MCF

$2,300,000$

$9,125,000$

$2,296,365$

$9,151,869$

$2,300,000$

$9,095,345$

$21,863,274$

$78,173,927$

(1) Acts as agent for MASSP.OWER and Granite State Gas Transmisslon System.
(2) Successor to NW-Alaskan Pipeline Co; gas sold to SoCal. (See order 1009-A). 
역

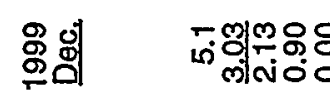

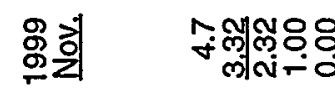

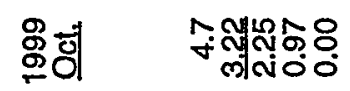

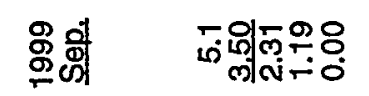

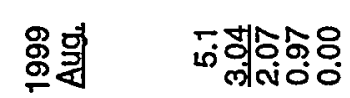

总司

용고

뜰롱

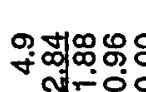

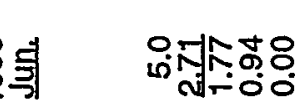

藏 통

웜워

द 을웅

u1 $0 \times \frac{\infty}{5}$

핑

ㄱํㄴ

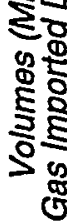

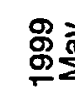

橸

호ㅇㅝㅓ

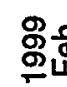

兽牙

o

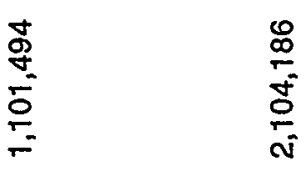

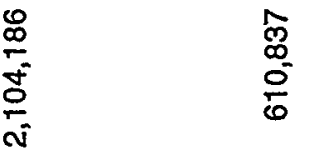

ㄴํํำํํํㅇำ

영잉웅유

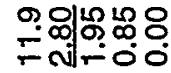

ㄱํำ

웅잉ㅇㅇㅇㅇㅇㅇ

웅잉ㅇㅇㅇㅇㅇㅇ

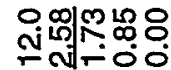

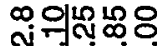

สูल

웅잉용ㅇㅇㅇ

엉엉ㅇㅇㅇㅇㅇㅇㅇ

어권ㅉㅇㅇㅇㅇ

엉건ㄴㅇㅇㅛ

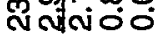

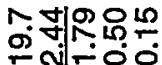

웅잉ㅇㅇㅇ웅

구쇼용요

위어ํํㅇㅇㅛ

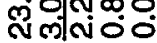

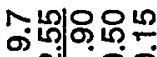

(1)

잉융유.

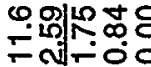

경유용요

다잉요

0 바용요

유용요

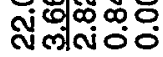

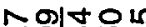

응잉ㅇㅇㅇㅇ

Nㅐㅇㅇㅇ

둥배뇽요

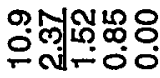

ถึज्ञ००

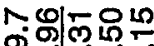

웅엉용ㅇㅇㅇ

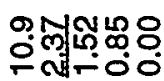

ํํำก용

(1)

웅잉ㅇㅇㅇㅇㅇ

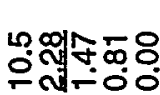

政

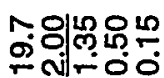

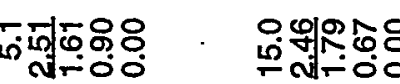

엤ํํำ

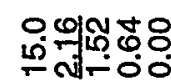

뚱뮈융ㅇㅇㅇ

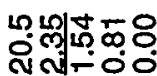

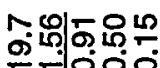

\section{Nल⿵人一}

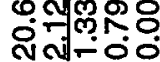

ำเำㅇํㅇำ:

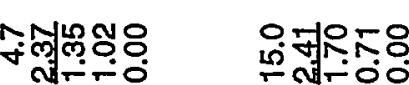

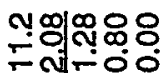

พั่ํำำ용

लNं०

긍궈ำㅇํㅇ

누ㄱㅝㅜㅇㅇㅇㅇ

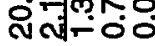

क.

稁䒺

$\stackrel{5}{\ddagger}$

$\stackrel{\infty}{=}$

มี่

ஷั่
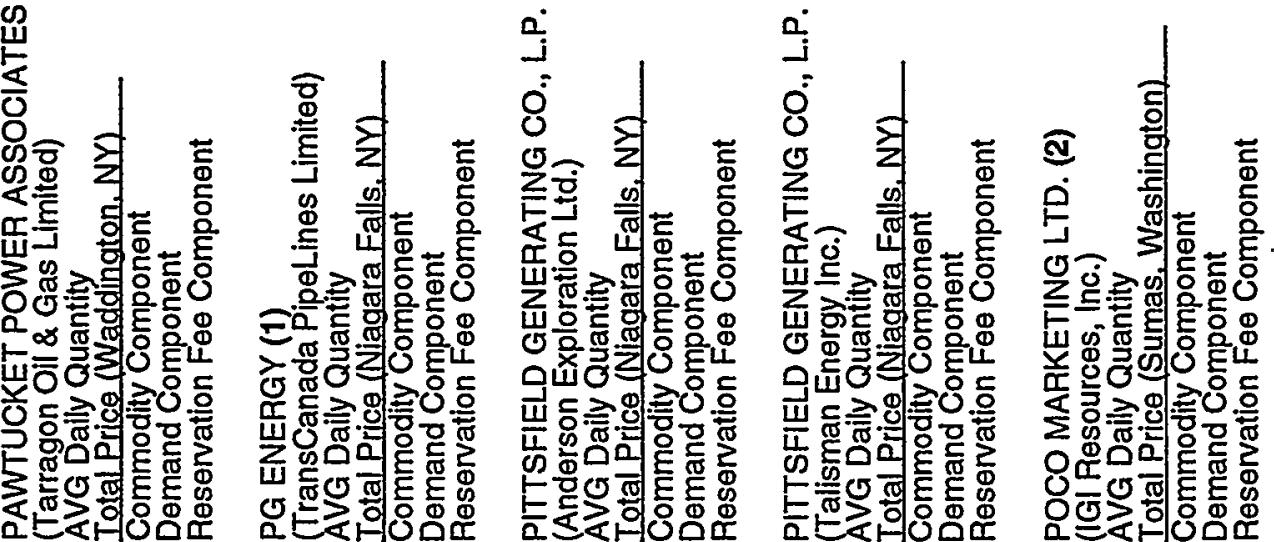


\section{VOLUME AND PRICE REPORT}

\section{Long-Term Imports}

Volumes (MMcf/d) \& Prices ( $\$$ MMBTU) of Natural

- Gas Imported During the Past 12 Months 01/01/99 - 12/31/99

Leng-Term Importer

\section{PORTAL MUNICIPAL GAS (1)}

(Sask Energy Inc.)

Total Price (Portal. North Dakota)

Commodity Component

Demand Component

Reservation Fee Component

PROGAS U.S.A. INC. (2)

ProGas Limited)

AVG Daily Quantity

Total Price (Noyes, Minnesota)

Commodity Component

Demand Component

Reservation Feo Component

PROGAS U.S.A. INC. (3)

(ProGas Limited)

AVG Daily Quantity

Total Price (Noves, Minnesota)

Commodity Component

Demand Component

Reservation Fee Component

PROGAS U.S.A. INC. (4)

(ProGas Limited)

AVG Daily Quantity

Total Price (Noyes, Minnesota)

Commodity Componen

Demand Component

Reservation Fee Component

PROGAS U.S.A. INC. (5)

(ProGas Limited)

AVG Daily Quantity

Total Price (Noyes. Minnesota)

Commodity Component

Demand Component

Reservation Fee Component
Auth. $\begin{array}{llllll}1999 & 1999 & 1999 & 1999 \cdot 1999 & 1999\end{array}$

2.3

\begin{tabular}{|c|c|c|c|c|c|c|c|c|c|c|}
\hline $\begin{array}{r}0.1 \\
2.36 \\
2.36 \\
0.00 \\
0.00\end{array}$ & $\begin{array}{r}0.1 \\
4.07 \\
4.07 \\
0.00 \\
0.00\end{array}$ & $\begin{array}{r}0.1 \\
3.05 \\
3.05 \\
0.00 \\
0.00\end{array}$ & $\begin{array}{r}0.0 \\
2.71 \\
2.71 \\
0.00 \\
0.00\end{array}$ & $\begin{array}{r}0.0 \\
2.80 \\
2.80\end{array}$ & $\begin{array}{r}0.0 \\
3.23 \\
3.23 \\
0.00 \\
0.00\end{array}$ & $\begin{array}{r}0.0 \\
3.51 \\
3.51 \\
0.00 \\
0.00\end{array}$ & $\begin{array}{r}0.0 \\
\frac{3.43}{3.43} \\
3.43\end{array}$ & $\begin{array}{r}0.0 \\
2.89 \\
2.89 \\
0.00 \\
0.00\end{array}$ & $\begin{array}{r}0.0 \\
2.72 \\
2.72 \\
0.00 \\
0.00\end{array}$ & $\begin{array}{r}0.0 \\
2.67 \\
2.67 \\
0.00 \\
0.00\end{array}$ \\
\hline
\end{tabular}

85.0

$\begin{array}{lll}85.1 & 85.1 & 85.1 \\ 2.60 & 2.56 & 2.52 \\ 1.85 & 1.81 & 1.77 \\ 0.75 & 0.75 & 0.75 \\ 0.00 & 0.00 & 0.00\end{array}$

85.1

85.1
2.24
1.49
0.75

85.1
2.24
1.49
0.75
0.00

85.1

0.00

2.6
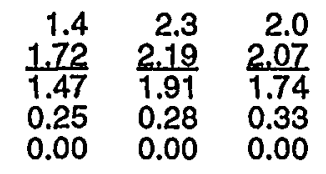

2.0
2.83
2.50
0.33
0.00
12.8

$\begin{array}{lll}12.9 & 12.9 & 12.9 \\ 2.61 & 2.68 & 2 \\ 2.02 & 2.02 & 2.02 \\ 0.59 & 0.66 & 0.59 \\ 0.00 & 0.00 & 0.00\end{array}$

26.5

$\begin{array}{rrr}1.3 & 1.3 & 1.3 \\ 2.23 & 2.30 & 2.23 \\ 1.75 & 1.76 & 1.75 \\ 0.48 & 0.54 & 0.48\end{array}$

0.00

$\begin{array}{lll}24.7 & 24.7 & 24.7 \\ \frac{1.98}{1.98} & \frac{1.96}{1.96} & \frac{1.88}{1.88} \\ 0.00 & 0.00 & 0.00 \\ 0.00 & 0.00 & 0.00\end{array}$

0.00

2.3
2.19
1.91
0.28
0.00

1.7
2.14
1.76
0.38
0.00

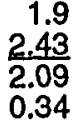

1.9
2.43
2.09
0.34
0.00
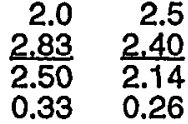

2.5
2.40
2.14
0.26
0.00

2.6
2.79
2.55
0.24

0.00 $\begin{array}{r}2.6 \\ 2.33 \\ \hline .08\end{array}$

2.08

0.25
0.00

$\begin{array}{ll}12.9 & \\ 2.61 & 1 \\ 2.02 & 1.51 \\ 0.59 & 0 \\ 0.00 & 0.00 \\ & \\ & \\ 24.7 & 2 \\ 1.88 & 2 \\ 1.88 & 2 \\ 0.00 & 0 . \\ 0.00 & 0.00\end{array}$

7.0
1.88
1.51
0.37
0.00


24.7
2.05
2.05
0.00
0.00

\begin{tabular}{rr}
7.2 & 7.7 \\
2.66 & 2.56 \\
\hline 1.97 & 1.90
\end{tabular}

1.97

0.69
0.00

$\begin{array}{ll}0.00 & 0.00\end{array}$

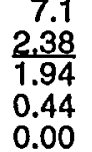

7.1
2.95

2.95
2.28
0.67

0.67
0.00

$\begin{array}{r}4.8 \\ 3.57 \\ \hline\end{array}$

$\frac{3.57}{2.54}$

1.03

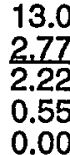

11.0

$\frac{3.47}{2.75}$

13.0

2.47
1.88

1.88
0.59

$\begin{array}{ll}0.72 & 0.59 \\ 0.00 & 0.00\end{array}$

\begin{tabular}{lll}
16.7 & 15.8 & 14.8 \\
2.53 & 2.40 & 2.48 \\
\hline 2.53 & $\frac{2.40}{2.48}$ \\
0.00 & 0.00 & 0.00 \\
0.00 & 0.00 & 0.00
\end{tabular}

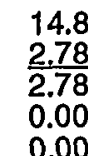

\begin{tabular}{l}
14.8 \\
2.78 \\
\hline 2.78 \\
0.00 \\
0.00
\end{tabular}

\begin{tabular}{l}
14.8 \\
.3 .17 \\
\hline 3.17 \\
0.00 \\
0.00
\end{tabular}

14.8
$\frac{2.81}{2.81}$
0.00
0.00

14.8
3.21
3.21
0.00
0.00

14.8
2.34
2.34
0.00
0.00

$1,363,910$

$6,684,539$

(1) Volumes often are too small to be shown on a monthly basis

3) Supply is resold to ConsumersEnergy Company. Dally contract volume specified in Order 1197-B

4) Supplles are resold to Great Plalns Natural Gas Company. 


\section{VOLUME AND PRICE REPORT}

\section{Long-Term Imports}

Volumes (MMcf/d) \& Prices (\$/MMBTU) of Natural Gas Imported During the Past 12 Months 01/01/99 - 12/31/99

Long-Term Importer

PROGAS U.S.A. INC. (1)

(ProGas Limited)

AVG Daily Quantity

Total Price (Port of Morgan. MT)

Commodity Component

Reservation Fee Component

PROGAS U.S.A. INC. - (DYNEGY)

(ProGas Ltd.)

AVG Daily Quantity

Total Price (Port of Morgan. MT)

Commodity Component

Demand Component

Reservation Feo Component

PROGAS U.S.A. INC. - (TENASKA GAS COMPANY)

(ProGas Limited)

AVG Daily Quantity

Total Price (Port of Morgan. MT)

Commodity Component

Demand Component

Reservation Fee Component

Auth.
Vols.

Jan. Fob.

Apr.

30.0

$\begin{array}{llll}24.0 & 23.2 & 29.1 & 24.3 \\ 1.88 & \frac{1.84}{1.19} & \frac{1.19}{1.19} & \frac{1.79}{1.79} \\ 1.88 & 1.84 & 2.00 & \\ 0.00 & 0.00 & 0.00 & 0.00 \\ 0.00 & 0.00 & 0.00 & 0.00\end{array}$

24.0
2.24
2.24
0.00
0.00

24.5

$\frac{2.16}{2.16}$

0.00

0.00

30.0

\begin{tabular}{lll}
28.8 & 28.7 & 29.0 \\
1.67 & 1.67 & 1.48 \\
\hline 1.20 & 1.20 & 1.02 \\
0.47 & 0.47 & 0.46
\end{tabular}

0.47

0.00

0.00

20.0

$\begin{array}{lll}19.2 & 18.9 & 1 \\ \frac{1.67}{1.16} & \frac{1.67}{1.15} & \frac{1}{0.97} \\ 0.51 & 0.52 & 0.50 \\ 0.00 & 0.00 & 0.00\end{array}$

29.4

$\begin{array}{ll}29.3 & 29.2 \\ 2.30 & 2.28 \\ 2.30 & 2.28 \\ 0.00 & 0.00 \\ 0.00 & 0.00\end{array}$

28.1
2.03
2.03
0.00
0.00

29.0
2.05
2.05
0.00
0.00

$\begin{array}{lr}29.4 & 9.0 \\ 2.49 & 2.42 \\ 2.49 & 2.42 \\ 0.00 & 0.00 \\ 0.00 & 0.00\end{array}$

29.2

Total Price (Waddington. NY)

Commodity Compone

Reservation Fee Component

PROGAS U.S.A., INC (3)

(Progas U.S.A. Inc.)

AVG Daily Quantity

Total Price (Eastport, Idaho)

Commodity Component

Demand Component

Reservation Fee Component
7.8

$\begin{array}{rrrr}7.2 & 7.2 & 5.7 & 7.1 \\ \frac{2.24}{2.24} & \frac{1.76}{1.76} & \frac{1.65}{1.65} & \frac{1.75}{1.75} \\ 0.00 & 0.00 & 0.00 & 0.00 \\ 0.00 & 0.00 & 0.00 & 0.00\end{array}$

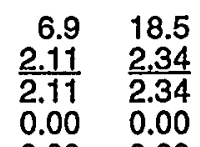

$0.00 \quad 0.00$
19991999

Jul.
(Progas U.S.A. Inc.)

91999

1999

19991999

ov. Dec.

2 Supplles are markeled in the Northeast, primarily in New York State. Dally contract quantity increase from 16.4 to 29.4 approved in Order 1206-A.

Supplies are markeled in California. 
Long-Term Importer

PROJECT ORANGE ASSOCIATES, L.P. (1)

(Noranda, Inc.)

AVG Daily Quantity

Total Price (Niagara Falls, NY)

Commodity Component

Demand Component

Reservation Fee Component

PUBLIC SERVICE ELECTRIC \& GAS

(Alberta NE Gas (TCGS Ltd. 1))

AVG Daily Quantity

Total Price (Waddington. NY)

Commodity Component

Demand Component

Reservation Fee Component

PUGET SOUND ENERGY, INC.

(Amoco Canada)

AVG Daily Quantity

Total Price (Sumas, Washington)

Commodity Component

Demand Component

Reservation Fee Component

PUGET SOUND ENERGY, INC.

(Duke Energy)

AVG Daily Quantity

Total Price (Sumas, Washington)

Commodity Component

Demand Component

Reservation Fee Component

PUGET SOUND ENERGY, INC.

(Engage Energy Canada)

AVG Daily Quantity

Total Price (Sumas, Washington)

Commodity Component

Reservation Fee Component

\section{VOLUME AND PRICE REPORT}

\section{Long-Term Imports}

Volumes (MMcf/d) \& Prices (\$/MMBTU) of Natural Gas Imported During the Past 12 Months 01/01/99 - 12/31/99
Auth. $1999 \quad 1999 \cdot 1999 \quad 1999$

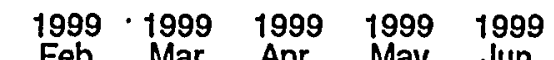

0.0

\begin{tabular}{ll}
17.5 & 14.2 \\
2.42 & 2.76 \\
\hline 0.73 & 0.73 \\
1.69 & 2.03 \\
0.00 & 0.00
\end{tabular}

10.0

$\begin{array}{lll}10.0 & 10.0 & 10.0 \\ 1.99 & 1.93 & \frac{1.88}{1.15} \\ 1.26 & 1.22 & 1.73 \\ 0.73 & 0.71 & 0.73 \\ 0.00 & 0.00 & 0.00\end{array}$

$\begin{array}{lll}0.00 & 0.00 & 0.00\end{array}$

24.0

$\begin{array}{llll}25.0 & 25.0 & 25.0 & 25.0 \\ 2.92 & 1.81 & 1.54 & \frac{1.55}{0.91} \\ 2.12 & 1.06 & 0.94 & 0.64 \\ 0.80 & 0.75 & 0.60 & 0.64\end{array}$

$\begin{array}{llll}0.80 & 0.75 & 0.60 & 0.64 \\ 0.00 & 0.00 & 0.00 & 0.00\end{array}$

14.5

$\begin{array}{lll}15.0 & 15.0 & 15.0 \\ 2.92 & 1.81 & \frac{1.54}{1.00} \\ 2.37 & 1.21 & 1.00 \\ 0.55 & 0.60 & 0.54 \\ 0.00 & 0.00 & 0.00\end{array}$

15.0
1.55
0.98
0.57
0.00

9.6

$\begin{array}{lll}10.0 & 10.0 & 10.0 \\ \frac{2.96}{2.35} & \frac{1.84}{1.19} & \frac{1.55}{1.02} \\ 0.61 & 0.65 & 0.53 \\ 0.00 & 0.00 & 0.00\end{array}$

\begin{tabular}{l}
10.0 \\
1.59 \\
\hline 1.02 \\
0.57 \\
0.00
\end{tabular}
15.0
1.99
1.42
0.57
0.00

15.0
1.95
1.37
0.58
0.00

15.0

$\frac{1.98}{1.46}$

$\begin{array}{lll}25.0 & 25.0 & 25.0\end{array}$

$\frac{1.99}{1.29} \quad \frac{1.95}{1.24} \quad \frac{1.98}{1.29}$

$\begin{array}{lll}1.29 & 1.24 & 1.29 \\ 0.70 & 0.71 & 0.69\end{array}$

$\begin{array}{lll}0.00 & 0.00 & 0.00\end{array}$

25.0
2.25

$\frac{2.25}{1.56}$

0.69
0.00

25.0
2.54

1.81

0.73
0.00

24.2
2.54
1.81

1.81

0.73
0.00

24.0
2.95
2.19

2.19
0.76

0.00

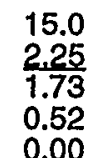

15.0
2.54
2.00
0.54
0.00

14.5
2.54
2.00
0.54
0.00

14.6
2.96
2.42
0.54

14.5

$\frac{2.31}{1.78}$

0.53

$\begin{array}{llllll}0.00 & 0.00 & 0.00 & 0.00 & 0.00 & 0.00\end{array}$

\begin{tabular}{l}
10.0 \\
2.06 \\
\hline 1.44 \\
0.62 \\
0.00
\end{tabular}

10.0
2.02
1.38
0.64
0.00

10.0
2.03
1.42
0.61
0.00

10.0
2.31
1.69
0.62
0.00

9.9
2.64
1.99
0.65

9.6
2.64
1.99
0.65

\begin{tabular}{l}
19.8 \\
2.96 \\
\hline 2.32 \\
0.64
\end{tabular}

\begin{tabular}{r}
4.8 \\
2.31 \\
\hline 1.87 \\
0.44
\end{tabular}

$1,041,599$

$3,768,221$

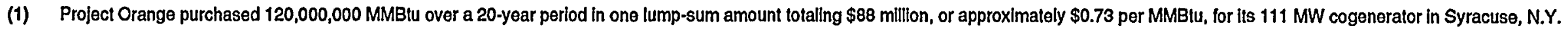




\section{VOLUME AND PRICE REPORT}

\section{Long-Term Imports}

Volumes (MMcf/d) \& Prices (\$/MMBTU) of Natural

Gas Imported During the Past 12 Months 01/01/99 - 12/31/99

Long-Term importer

PUGET SOUND ENERGY, INC.

(Engage Energy Canada)

AVG Daily Quantity

Total Price (Eastport. Idaho)

Commodity Component

Demand Component

Reservation $\mathrm{Fee}$ Component

PUGET SOUND ENERGY, INC.

(Poco Petroleums Ltd.)

AVG Daily. Quantity

Total Price (Sumas, Washington) '

Commodity Component

Demand Component

Reservation Fee Component

RENAISSANCE ENERGY (U.S.) INC. (1)

(Renaissance Energy Limited)

AVG Daily Quantity

Total Price (Port of Morgan, MT)

Commodity Component

Demand Component

Reservation Fee Component

RENAISSANCE ENERGY (U.S.) INC. (2)

(Renaissance Energy Limited)

AVG Daily Quantity

Total Price (Niagara Falls, NY)

Commodity Component

Demand Component

Reservation $\mathrm{Fe \theta}$ Component

RENAISSANCE ENERGY (U.S.) INC. (3)

(Renaissance Energy Limited)

AVG Daily Quantity

Total Price (Niagara Falls, NY)

Commodity Componen

Demand Component

Reservation $\mathrm{Fee}$ Component $\begin{array}{lllllllllllll}\text { Auth. } & 1999 & 1999 & 1999 & 1999 & 1999 & 1999 & 1999 & 1999 & 1999 & 1999 & 1999 & 1999\end{array}$

Vols. Jan Eeb Mar. Apr. Mav, Jun Jul. Aug Sep. Oct. Nov Dec.

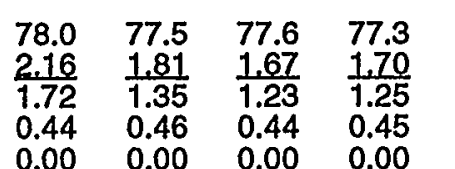

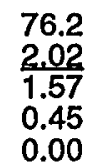

77.9
2.01
1.56
0.45
0.00

77.5
2.04
1.60
0.44

77.9
2.1
1.71
0.44
0.00

76.0

$0.00 \quad 0.00$

14.4

$\begin{array}{lll}15.0 & 15.0 & 15.0 \\ \frac{2.92}{2.25} & \frac{1.81}{1.14} & \frac{1.54}{1.01} \\ 0.67 & 0.67 & 0.53 \\ 0.00 & 0.00 & 0.00\end{array}$

15.0
1.55
0.99
0.56
0.00

15.0

$\frac{1.99}{1.38}$

0.61

0.00

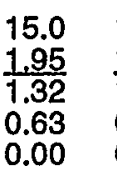

\begin{tabular}{l}
15.0 \\
1.98 \\
\hline 1.38 \\
0.60 \\
0.00
\end{tabular}

15.0
2.25
1.63
0.62
0.00

15.0
2.54
1.86
0.68

$\begin{array}{rrrr}5.1 & 5.1 & 5.1 & 5.1 \\ 1.84 & \frac{1.83}{1.39} & 1.39 & 1.44 \\ 1.84 & 1.83 & 1.39 & 1.44 \\ 0.00 & 0.00 & 0.00 & 0.00\end{array}$

5.1
1.85
1.85
0.00

5.1
$\frac{1.84}{1.84}$
0.00

5.1
1.86
1.86
0.00
0.00

$\begin{array}{r}5.0 \\ 1.98 \\ \hline 1.98\end{array}$

0.00

$0.00 \quad 0.00$

0.00

2.8

$\begin{array}{rrrr}2.8 & 2.8 & 2.8 & 2.8 \\ 2.31 & \frac{2.37}{1.53} & \frac{2.05}{1.27} & 2.35 \\ 1.55 \\ 0.78 & 0.89 & 0.78 & 0.80 \\ 0.00 & 0.00 & 0.00 & 0.00\end{array}$

2.8
2.80
2.02
0.78
0.00

$\begin{array}{ll}2.8 & 2.8 \\ 2.75 & \frac{2.75}{1.92} \\ 1.91 & \\ 0.84 & 0.83 \\ 0.00 & 0.00\end{array}$

2.8
$\frac{3.04}{2.21}$
0.83
0.00

\begin{tabular}{r}
2.8 \\
3.50 \\
\hline 2.63 \\
0.87 \\
0.00
\end{tabular}

$\begin{array}{r}2.8 \\ 3.21 \\ \hline\end{array}$

$\frac{3.21}{2.37}$

0.84
0.00

$\frac{3.66}{2.81}$

2.81
0.85

0.00

2.37

0.83

0.00

23.4

$\begin{array}{lll}15.2 & 18.6 & 16.5 \\ \frac{2.04}{2.04} & \frac{1.73}{1.73} & \frac{1.95}{1.95} \\ 0.00 & 0.00 & 0.00 \\ 0.00 & 0.00 & 0.00\end{array}$

0.0
0.00
0.00
0.00
0.00

\begin{tabular}{rr}
0.0 & 0.0 \\
$\underline{0.00}$ & 0.00 \\
\hline 0.00 & 0.00 \\
0.00 & 0.00 \\
0.00 & 0.00
\end{tabular}

0.0
0.00
0.00
0.00
0.00

0.0
0.00
0.00
0.00
0.00

0.0
0.00
0.00
0.00
0.00

0.0
0.00
0.00
0.00
0.00

13.3
3.1
3.1
0.00

15.2

2.40

2.40

0.00
4Q99

TOTAL MCA

YTD

IOTAL MCf

$6,778,620$

$27,885,067$

$1,349,422$

$5,444,345$

470,776

$1,855,056$

257,600

$1,022,000$

867,553

$2,369,426$

(1) Renaissance resells these volumes to AmGas Inc., for final sale to various customers in lowa.

(3) Supplies are sold to markels off the pipeline system of National Fuel Gas Supply Corporalion. This contract is for winter-only supplies. 


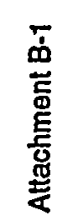

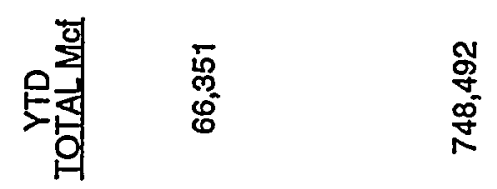

翼ㄴ 童

管。”。

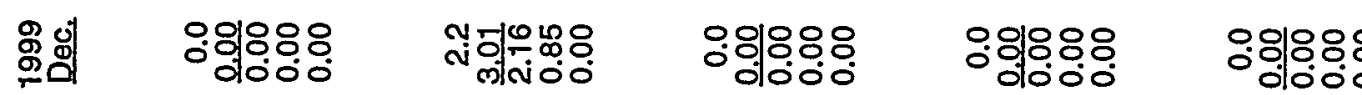

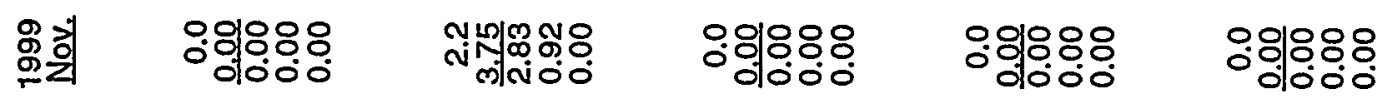

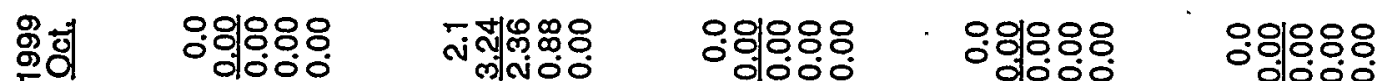

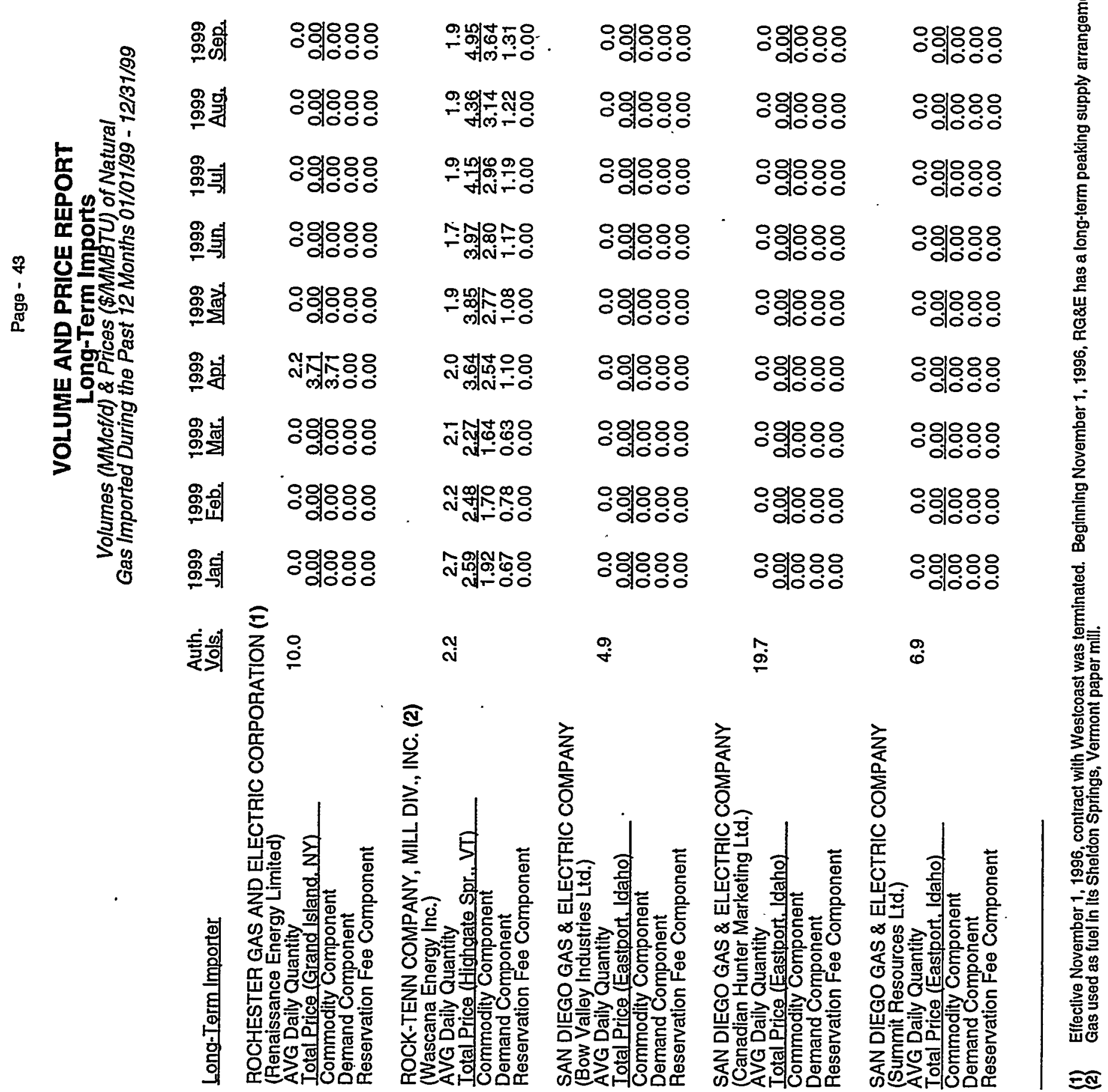




\section{VOLUME AND PRICE REPORT}

\section{Long-Term Imports}

Volumes (MMcf/d) \& Prices (\$MMBTU) of Natural

Gas Imported During the Past 12 Months 01/01/99 - 12/31/99

\section{Long-Term Importer}

SARANAC POWER PARTNERS, L.P.

(Shell Canada Limited)

AVG Daily Quantity

Commodity Component

Demand Component

Reservation Fee Component

SELKIRK COGEN PARTNERS, L.P.

(Imperial Oil Resources Limited)

AVG Daily Quantity

Total Price (Waddington. NY)

Commodity Component

Demand Component

Reservation Fee Component

\section{SELKIRK COGEN PARTNERS, L.P.}

(PanCanadian Petroleum Ltd.)

AVG Daily Quantity

Total Price (Waddington. NY)

Commodity Component

SELKIRK COGEN PARTNERS, L.P. (1)

(Paramount Resources Ltd.)

AVG Daily Quantity

Commodity Component

Demand Component

Reservation Fee Component

SELKIRK COGEN PARTNERS, L.P.

\section{(Producers Marketing, Ltd.)}

AVG Daily Quantity

Total Price (Waddington, NY)

Commodity Component

Demand Component

Reservation Fee Component
Total Price (Champlain. NY)

Demand Component

Reservation Fee Component

Total Price (Waddington. NY)

\begin{tabular}{|c|c|c|c|c|c|c|c|c|c|c|c|c|c|c|}
\hline $\begin{array}{l}\text { Auth. } \\
\text { Vols. }\end{array}$ & $\begin{array}{l}1999 \\
\text { Jan. }\end{array}$ & $\begin{array}{l}1999 \\
\text { Feb. }\end{array}$ & $\begin{array}{l}1999 \\
\text { Mar. }\end{array}$ & $\begin{array}{l}1999 \\
\text { Apr. }\end{array}$ & $\begin{array}{l}1999 \\
\text { Mav. }\end{array}$ & $\begin{array}{l}1999 \\
\text { Jun. }\end{array}$ & $\begin{array}{l}1999 \\
\text { Jull. }\end{array}$ & $\begin{array}{l}1999 \\
\text { Aug. }\end{array}$ & $\begin{array}{l}1999 \\
\text { Sep. }\end{array}$ & $\begin{array}{l}1999 \\
\text { Qㄷ. }\end{array}$ & $\begin{array}{l}1999 \\
\text { Nov. }\end{array}$ & $\begin{array}{l}1999 \\
\text { Dec. }\end{array}$ & $\begin{array}{l}4099 \\
\text { TOTALMCf }\end{array}$ & $\begin{array}{l}\text { YTD } \\
\text { IOTALMCF }\end{array}$ \\
\hline 54.0 & $\begin{array}{l}46.2 \\
3.61 \\
2.82 \\
0.79 \\
0.00\end{array}$ & $\begin{array}{l}44.4 \\
3.61 \\
2.72 \\
0.89 \\
0.00\end{array}$ & $\begin{array}{l}43.7 \\
\frac{3.61}{2.82} \\
0.79 \\
0.00\end{array}$ & $\begin{array}{l}45.4 \\
\frac{3.61}{2.78} \\
0.83 \\
0.00\end{array}$ & $\begin{array}{l}44.4 \\
3.61 \\
2.79 \\
0.82 \\
0.00\end{array}$ & $\begin{array}{l}44.8 \\
3.62 \\
2.77 \\
0.85 \\
0.00\end{array}$ & $\begin{array}{l}46.7 \\
3.62 \\
2.81 \\
0.81 \\
0.00\end{array}$ & $\begin{array}{l}46.3 \\
\frac{3.61}{2.81} \\
0.80 \\
0.00\end{array}$ & $\begin{array}{l}45.3 \\
\frac{3.61}{2.77} \\
0.84 \\
0.00\end{array}$ & $\begin{array}{l}45.4 \\
\frac{3.61}{2.80} \\
0.81 \\
0.00\end{array}$ & $\begin{array}{l}44.5 \\
\frac{3.75}{2.91} \\
0.84 \\
0.00\end{array}$ & $\begin{array}{l}44.8 \\
3.76 \\
2.94 \\
0.82 \\
0.00\end{array}$ & $4,132,738$ & $16,490,874$ \\
\hline 19.0 & $\begin{array}{l}19.0 \\
2.34 \\
1.27 \\
1.07 \\
0.00\end{array}$ & $\begin{array}{l}19.0 \\
2.24 \\
1.19 \\
1.05 \\
0.00\end{array}$ & $\begin{array}{l}19.0 \\
2.03 \\
1.00 \\
1.03 \\
0.00\end{array}$ & $\begin{array}{l}19.8 \\
2.23 \\
1.23 \\
1.00 \\
0.00\end{array}$ & $\begin{array}{l}20.4 \\
2.52 \\
1.51 \\
1.01 \\
0.00\end{array}$ & $\begin{array}{l}20.4 \\
2.53 \\
1.46 \\
1.07 \\
0.00\end{array}$ & $\begin{array}{l}19.0 \\
2.31 \\
1.25 \\
1.06 \\
0.00\end{array}$ & $\begin{array}{l}19.0 \\
2.45 \\
1.40 \\
1.05 \\
0.00\end{array}$ & $\begin{array}{l}19.0 \\
2.63 \\
1.58 \\
1.05 \\
0.00\end{array}$ & $\begin{array}{l}19.0 \\
2.57 \\
\frac{2.52}{1.52} \\
1.05 \\
0.00\end{array}$ & $\begin{array}{l}19.0 \\
2.77 \\
1.71 \\
1.06 \\
0.00\end{array}$ & $\begin{array}{l}19.0 \\
2.49 \\
1.43 \\
1.06 \\
0.00\end{array}$ & $1,747,908$ & $7,045,183$ \\
\hline 19.0 & $\begin{array}{l}19.0 \\
2.34 \\
1.28 \\
1.06 \\
0.00\end{array}$ & $\begin{array}{l}19.0 \\
2.17 \\
1.13 \\
1.04 \\
0.00\end{array}$ & $\begin{array}{l}19.0 \\
1.99 \\
0.97 \\
1.02 \\
0.00\end{array}$ & $\begin{array}{l}19.6 \\
2.04 \\
1.05 \\
0.99 \\
0.00\end{array}$ & $\begin{array}{l}20.5 \\
2.30 \\
1.29 \\
1.01 \\
0.00\end{array}$ & $\begin{array}{l}20.4 \\
2.32 \\
1.27 \\
1.05 \\
0.00\end{array}$ & $\begin{array}{l}19.0 \\
2.31 \\
1.26 \\
1.05 \\
0.00\end{array}$ & $\begin{array}{l}19.0 \\
2.46 \\
1.42 \\
1.04 \\
0.00\end{array}$ & $\begin{array}{l}19.0 \\
2.63 \\
1.60 \\
1.03 \\
0.00\end{array}$ & $\begin{array}{l}19.0 \\
2.58 \\
1.54 \\
1.04 \\
0.00\end{array}$ & $\begin{array}{l}19.0 \\
2.77 \\
1.73 \\
1.04 \\
0.00\end{array}$ & $\begin{array}{l}19.0 \\
2.49 \\
1.44 \\
1.05 \\
0.00\end{array}$ & $1,747,908$ & $7,042,173$ \\
\hline 16.4 & $\begin{array}{l}15.1 \\
2.26 \\
1.59 \\
0.67 \\
0.00\end{array}$ & $\begin{array}{l}15.1 \\
2.34 \\
1.69 \\
0.65 \\
0.00\end{array}$ & $\begin{array}{l}15.1 \\
2.08 \\
1.44 \\
0.64 \\
0.00\end{array}$ & $\begin{array}{l}16.2 \\
2.52 \\
1.70 \\
0.82 \\
0.00\end{array}$ & $\begin{array}{l}16.3 \\
2.85 \\
2.00 \\
0.85 \\
0.00\end{array}$ & $\begin{array}{l}16.3 \\
2.82 \\
1.95 \\
0.87 \\
0.00\end{array}$ & $\begin{array}{l}15.1 \\
2.83 \\
1.95 \\
0.88 \\
0.00\end{array}$ & $\begin{array}{l}15.1 \\
2.98 \\
\frac{2.12}{2.12} \\
0.86 \\
0.00\end{array}$ & $\begin{array}{l}15.1 \\
3.34 \\
2.47 \\
0.87 \\
0.00\end{array}$ & $\begin{array}{l}15.1 \\
\frac{3.26}{2.38} \\
0.88 \\
0.00\end{array}$ & $\begin{array}{l}15.1 \\
\frac{3.76}{2.88} \\
0.88 \\
0.00\end{array}$ & $\begin{array}{l}14.1 \\
2.92 \\
2.05 \\
0.87 \\
0.00\end{array}$ & $1,358,524$ & $5,585,441$ \\
\hline 17.0 & $\begin{array}{l}16.9 \\
2.28 \\
1.32 \\
0.96 \\
0.00\end{array}$ & $\begin{array}{l}16.9 \\
2.12 \\
1.17 \\
0.95 \\
0.00\end{array}$ & $\begin{array}{l}16.9 \\
1.94 \\
1.02 \\
0.92 \\
0.00\end{array}$ & $\begin{array}{l}17.5 \\
1.99 \\
1.10 \\
0.89 \\
0.00\end{array}$ & $\begin{array}{l}18.3 \\
2.24 \\
1.33 \\
0.91 \\
0.00\end{array}$ & $\begin{array}{l}18.3 \\
2.27 \\
1.30 \\
0.97 \\
0.00\end{array}$ & $\begin{array}{l}16.9 \\
2.24 \\
1.29 \\
0.95 \\
0.00\end{array}$ & $\begin{array}{l}16.9 \\
2.38 \\
1.44 \\
0.94 \\
0.00\end{array}$ & $\begin{array}{l}16.9 \\
\frac{2.55}{1.61} \\
0.94 \\
0.00\end{array}$ & $\begin{array}{l}16.9 \\
2.51 \\
1.56 \\
0.95 \\
0.00\end{array}$ & $\begin{array}{l}16.9 \\
2.69 \\
1.74 \\
0.95 \\
0.00\end{array}$ & $\begin{array}{l}16.9 \\
2.42 \\
1.47 \\
0.95 \\
0.00\end{array}$ & $1,555,645$ & $6,272,457$ \\
\hline
\end{tabular}

(1) As a result of restructuring its power sales agreement wilh Niagara Mohawk Power Corp., Selkink's daily contract demand was reduced to $16.4 \mathrm{MMcl}$, effective 8/31/98. 


\section{VOLUME AND PRICE REPORT}

\section{Long-Term Imports}

Volumes (MMcf/d) \& Prices (\$MMBTU) of Natural

Gas Imported During the Past 12 Months 01/01/99 - 12/31/99

Long-Term Importer

SIERRA PACIFIC POWER COMPANY

(Amoco Canada)

AVG Daily Quantity

Total Price (Eastport. Idaho)

Commodity Component

Demand Component

Reservation Fee Component

$1999 \quad 1999$

Jan

15.0

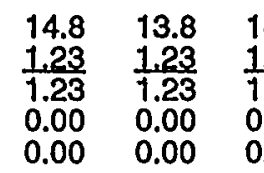

14.8
1.23
1.23
0.00
0.00

14.8
1.34
1.34
0.00
0.00

14.8
1.34
1.34
0.00
0.00

14.8
1.34
1.34
0.00
0.00

\begin{tabular}{l}
14.8 \\
1.34 \\
\hline 1.34 \\
0.00 \\
0.00
\end{tabular}

14.8
1.34
1.34
0.00
0.00

SITHEINDEPENDENCE POWER PARTNERS, L.P.

(Enron Power Services Inc.)

AVG Daily Quantity

32.0

Tommodity (Grand sland. NY)

Demand Component

Reservation Fee Component

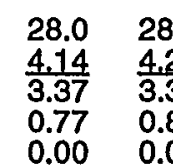

28.0
4.13
3.36
0.77
0.00

28.0
4.17
3.37
0.80
0.00

28.0

3.38

0.77

28.0

$\frac{4.16}{3.36}$

$\begin{array}{lll}0.00 & 0.00 & 0.00\end{array}$

15.0

\begin{tabular}{ll}
15.0 & 15.0 \\
1.64 & 1.58 \\
\hline 1.64 & 1.58 \\
0.00 & 0.00
\end{tabular}

15.0
$\frac{1.52}{1.52}$
0.00

15.0
1.68
1.68
0.00

0.00

0.00

2.2

\begin{tabular}{rrr}
2.2 & 2.2 & 2.2 \\
1.90 & $\frac{1.95}{1.12}$ & $\frac{1.80}{0.98}$ \\
\hline 1.09 & 1.12 & 0.82 \\
0.81 & 0.83 & 0.82 \\
0.00 & 0.00 & 0.00
\end{tabular}

2.2
2.03
1.19
0.84

2.2
2.52
1.67

Alberta NE Gas (AE

Total Price (Waddington. NY)

Demand Component

Reservation Fee Component

SOUTHERN CONNECTICUT GAS

(Alberta NE Gas (TCGS Ltd. 1))

AVG Daily Quantity

Total Price (Waddington, NY)

Commodity Componen

Demand Component

Reservation Fee Component
15.0

$\begin{array}{lll}22.5 & 22.5 & 22.5 \\ \frac{1.99}{1.26} & \frac{1.93}{1.22} & \frac{1.88}{1.15} \\ 0.73 & 0.71 & 0.73 \\ 0.00 & 0.00 & 0.00\end{array}$

$\begin{array}{llll}22.5 & 22.5 & 22.5 & 22.5 \\ 2.04 & 2.51 & 2.39 & 2.46 \\ \frac{2.29}{1.29} & 1.76 & 1.60 & 1.68 \\ 0.75 & 0.75 & 0.79 & 0.78 \\ 0.00 & 0.00 & 0.00 & 0.00\end{array}$

22.5
$\frac{3.15}{2.36}$
0.79
0.00

\begin{tabular}{l}
22.5 \\
2.80 \\
\hline 2.01 \\
0.79 \\
0.00
\end{tabular}

\begin{tabular}{ll}
17.0 & 22.5 \\
3.22 & 2.36 \\
\hline 2.43 & 1.57 \\
0.79 & 0.79 \\
0.00 & 0.00
\end{tabular}
22.5
2.76
1.98
0.78
0.00
14.8
1.34
1.34
0.00
0.00

14.8
1.34
1.34
0.00
0.00

14.6
1.34

4Q99

TOTALMC

TOTALM

$1,357,965$

$5,378,739$

$\frac{1.34}{1.34}$

0.00

$0.00 \quad 0.00$

$\begin{array}{llllll}.0 & 28.0 & 26.1 & 28.0 & 28.0 & 28.0 \\ 36 & \frac{4.13}{3.36} & \frac{4.22}{3.37} & \frac{4.15}{3.38} & \frac{4.18}{3.37} & \frac{4.16}{3.37} \\ 77 & 0.77 & 0.85 & 0.77 & 0.81 & 0.79 \\ 0.00 & 0.00 & 0.00 & 0.00 & 0.00 & 0.00\end{array}$

$2,576,727$

$10,166,868$

0.00

$1,381,748$

$5,497,996$

164,920

757,330

$1,905,000$

$8,047,500$ 
VOLUME AND PRICE REPORT

\section{Long-Term Imports}

Volumes (MMcf/d) \& Prices (\$MMBTU) of Natural Gas Imported During the Past 12 Months 01/01/99 - 12/31/99

Long-Term Importer

Auth. $\quad 1999 \quad 1999 \quad 1999 \quad 1999$

Vols.

$\begin{array}{lllll}1999 & 1999 & 1999 & 1999\end{array}$

1999

SOUTHERN CONNECTICUT GAS

(Alberta NE Gas (TCGS Ltd. 2))

AVG Daily Quantity

Total Price (Waddington. NY)

Commodity Component

Demand Component

Reservation Fee Component

SOUTHERN CONNECTICUT GAS

(Alberta Northeast Gas (ProGas))

AVG Daily Quantity

Total Price (Waddington. NY)

Commodity Component

Demand Component

Reservation Fee Component

SOUTHERN CONNECTICUT GAS

(Alberta Northeast Gas (Producers Marketing))

AVG Daily Quantity

Total Price (Waddington. NY)

Commodity Component

Demand Component

Reservation Fee Component

ST. LAWRENCE GAS COMPANY, INC. (1)

(TCGS Ltd.)

AVG Daily Quantity

Total Price (Massena, New York)

Commodity Component

Demand Component

Reservation Fee Component

STATOIL ENERGY, INC. (2)

(Talisman Energy Inc.)

AVG Daily Quantity

Total Price (Sumas, Washington)

Commodity Component

Demand Component

Reservation Fee Component
7.5

\begin{tabular}{|c|c|c|c|c|c|c|c|c|c|c|c|}
\hline $\begin{array}{r}0.0 \\
0.00 \\
0.00 \\
0.00 \\
0.00\end{array}$ & $\begin{array}{r}0.0 \\
0.00 \\
0.00 \\
0.00 \\
0.00\end{array}$ & $\begin{array}{r}0.0 \\
0.00 \\
0.00 \\
0.00 \\
0.00\end{array}$ & $\begin{array}{r}0.0 \\
0.00 \\
0.00 \\
0.00 \\
0.00\end{array}$ & $\begin{array}{r}0.0 \\
0.00 \\
0.00 \\
0.00 \\
0.00\end{array}$ & $\begin{array}{r}0.0 \\
0.00 \\
0.00 \\
0.00 \\
0.00\end{array}$ & $\begin{array}{r}0.0 \\
0.00 \\
0.00 \\
0.00 \\
0.00\end{array}$ & $\begin{array}{r}0.0 \\
0.00 \\
0.00 \\
0.00 \\
0.00\end{array}$ & $\begin{array}{r}0.0 \\
0.00 \\
0.00 \\
0.00 \\
0.00\end{array}$ & $\begin{array}{r}0.0 \\
0.00 \\
0.00 \\
0.00 \\
0.00\end{array}$ & $\begin{array}{r}0.0 \\
0.00 \\
0.00 \\
0.00 \\
0.00\end{array}$ & $\begin{array}{r}0.0 \\
0.00 \\
0.00 \\
0.00 \\
0.00\end{array}$ \\
\hline
\end{tabular}

6.6

\begin{tabular}{rr}
6.6 & 6.6 \\
1.92 & 1.96 \\
\hline 1.06 & 1.08 \\
0.86 & 0.88
\end{tabular}

0.86

0.00

0.00

6.6
1.80
0.93
0.87
0.00

6.6
$\frac{2.10}{1.25}$
0.85
0.00

6.6
253

$\frac{2.53}{1.63}$

0.90

0.00

\begin{tabular}{r}
6.6 \\
2.45 \\
\hline 1.52 \\
0.93 \\
0.00
\end{tabular}

6.6
$\frac{2.51}{1.60}$
0.91

6.6
$\frac{2.88}{1.97}$
0.91

6.6
$\frac{3.16}{2.24}$
0.92

6.6
2.99

2.07

0.92
0.00

\begin{tabular}{r}
2.6 \\
3.38 \\
\hline 2.48 \\
0.90
\end{tabular}

0.90
0.00

6.6
$\frac{2.79}{1.87}$
0.92

3.7

$\begin{array}{rrr}3.7 & 3.7 & 3.7 \\ 1.92 & 1.96 & 1.80 \\ 1.08 & \frac{1.11}{0.96} & 0.84 \\ 0.84 & 0.85 & 0.84 \\ 0.00 & 0.00 & 0.00\end{array}$

3.7
2.10
1.25
0.85
0.00

$\begin{array}{rrr}3.7 & 3.7 & 3.7 \\ 2.53 & 2.45 & 2.51 \\ 1.66 & 1.55 & 1.63 \\ 0.87 & 0.90 & 0.88 \\ 0.00 & 0.00 & 0.00\end{array}$

3.7
2.88
2.00
0.88
0.00

\begin{tabular}{rr}
3.7 & 3.7 \\
3.16 & 2.99 \\
\hline
\end{tabular}

2.27

0.89

3.7
2.99
2.10
0.89
0.00

2.6
3.37

$\frac{3.37}{2.44}$

2.44

$0.93 \quad 0.89$

$\begin{array}{llllllllllll}0.00 & 0.00 & 0.00 & 0.00 & 0.00 & 0.00 & 0.00 & 0.00 & 0.00 & 0.00 & 0.00 & 0.00\end{array}$

18.8

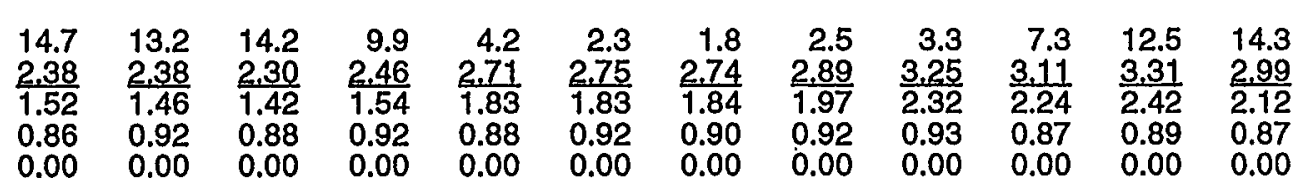

2.7

$\begin{array}{rrrr}2.7 & 2.4 & 1.9 & 2.0 \\ \frac{1.74}{1.74} & \frac{1.78}{1.78} & \frac{1.77}{1.77} & \frac{1.76}{1.76} \\ 0.00 & 0.00 & 0.00 & 0.00 \\ 0.00 & 0.00 & 0.00 & 0.00\end{array}$

\begin{tabular}{r}
3.0 \\
1.84 \\
\hline 1.84 \\
0.00 \\
0.00
\end{tabular}

2.8
$\frac{1.82}{1.82}$
0.00

2.6
$\frac{1.82}{1.82}$
0.00
0.00

2.6
$\frac{1.91}{1.91}$
0.00
0.00

2.3
$\frac{1.93}{1.93}$
0.00

$\frac{2.3}{1.91}$

$\frac{2.6}{2.08}$

$\begin{array}{lll}0.00 & 0.00 & 0.00 \\ 0.00 & 0.00 & 0.00\end{array}$

2.5
1.92
1.92
0.00
0.00
4099

TOTALMC

YOTAL

TIALM

0

0

487,955

$2,289,755$

310,015

$1,324,787$

$1,045,222$

$3,037,032$

226,167

902,387

(1) As the result of a contract amendment, daily contract volumes were reduced to 18.8 MMcf, effective January 1. 1997:

(2) Statoil Energy, Inc. was formerly known as Eastern Energy Marketling, Inc. Supply used to fuel a 10 Megawalt gas-fir,ed cogeneration facility near Glenns Ferny, Idaho. 


\section{VOLUME AND PRICE REPORT}

\section{ENg-Term Imports}

Volumes (MMcf/d) \& Prices (\$MMBTU) of Natural

Gas Imported During the Past 12 Months 01/01/99 - 12/31/99

Long-Term Importer

STATOIL ENERGY, INC. (1)

(Talisman Energy Inc.)

AVG Daily Quantity
Total Price (Sumas. Washington)

Commodity Component

Demand Component

Reservation $\mathrm{Fee}$ Component

SUMAS COGENERATION COMPANY, L.P. (2)

(ENCO Gas, Ltd.)

Total Price (Sumas, Washington)

Commodity Component

Demand Component

Reservation $\mathrm{Fee}$ Component

$\begin{array}{lr}22.3 & 0.7 \\ 2.17 & 2.28 \\ 2.17 & 2.28 \\ 0.00 & 0.00\end{array}$

3.5
2.28
2.28
0.00
0.00

0.6
2.28
2.28
0.00
0.00
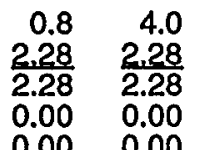

24.4
2.28
2.28
0.00

$0.00 \quad 0.00$

TENASKA WASHINGTON PARTNERS, L.P. (3)

(Puget Sound Energy, Inc.)

AVG Daily Quantity

Total Price (Sumas, Washington)

50.0

$\begin{array}{rrrrrrrrrrrr}0.0 & 0.0 & 0.0 & 0.0 & 0.0 & 0.0 & 0.0 & 0.0 & 0.0 & 0.0 & 0.0 & 0.0 \\ 0.00 & \frac{0.00}{0.00} & \frac{0.00}{0.00} & \frac{0.00}{0.00} & \frac{0.00}{0.00} & \frac{0.00}{0.00} & \frac{0.00}{0.00} & \frac{0.00}{0.00} & \frac{0.00}{0.00} & \frac{0.00}{0.00} & \frac{0.00}{0.00} & \frac{0.00}{0.00} \\ 0.00 & 0.00 & 0.00 & 0.00 & 0.00 & 0.00 & 0.00 & 0.00 & 0.00 & 0.00 & 0.00 & 0.00 \\ 0.00 & 0.00 & 0.00 & 0.00 & 0.00 & 0.00 & 0.00 & 0.00 & 0.00 & 0.00 & 0.00 & 0.00\end{array}$

Demand Component

Reservation Fee Component

THE U.S. GENERAL SERVICES ADMIN. (4)

(Chief Mountain Natural Gas Coop.)

AVG Daily Quantity

Total Price (Babb. Montana)

Commodity Compone

Demand Component

Reservation Fee Component

0.2

\begin{tabular}{rr}
0.0 & 0.0 \\
1.75 & 2.55 \\
\hline 1.75 & 2.55 \\
0.00 & 0.00 \\
0.00 & 0.00
\end{tabular}

0.00
0.00

0.00

$\begin{array}{rr}0.0 & 0.0 \\ 0 & 0\end{array}$

0.0
2.55
2.55
0.00

0.0
2.57
2.57
0.00

0.0
2.46
2.46
0.00

0.0
2.62
2.62
0.00

0.0
3.15
3.15

$\frac{3.15}{3.15}$

0.00

0.00

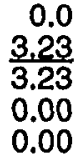

0.0
3.26
3.26

0.00

0.00

$0.0 \quad 0.0$

$\frac{3.11}{3.11} \quad \frac{3.19}{3.19}$

$3.11 \quad 3.19$

$0.00 \quad 0.00$

10.0

\begin{tabular}{rrrrrr}
9.8 & 10.0 & 8.5 & 8.5 & 7.3 & 7.5 \\
$\frac{2.32}{2.32}$ & $\frac{2.32}{2.32}$ & $\frac{2.32}{2.32}$ & $\frac{2.32}{2.32}$ & $\frac{2.32}{2.32}$ & $\frac{2.32}{2.32}$ \\
\hline 2.32 & 0.32 & 0.00 & 0.00 & 0.00 & 0.00 \\
0.00 & 0.00 & 0.00 & 0.00 & 0.00 & 0.00 \\
0.00 & 0.00 & 0.00 & 0.00 & 0
\end{tabular}

8.5
2.32
2.32
0.00
0.00

8.5
$\frac{2.32}{2.32}$
0.00
0.00

10.0

$\frac{2.32}{2.32}$

$10.0 \quad 10.0$

$\frac{2.32}{2.32}$

$\frac{2.32}{2.32}$

$\begin{array}{lll}0.00 & 0.00 & 0.32\end{array}$

$\frac{2.32}{2.32}$

$\begin{array}{lll}0.00 & 0.00 & 0.00 \\ 0.00 & 0.00 & 0.00\end{array}$

238,439

913,609

Reservation Fee Component

(1) Statoil Energy, Inc. was formerly kniown as Eastern'Energy Markeling, Inc. Supply used to fuel a 10 Megawatl gas-fired cogeneration facility near Rupert, Idaho. 


\section{VOLUME AND PRICE REPORT}

\section{Long-Term Imports}

Volumes (MMcf/d) \& Prices ( $\$ M M M B T U$ ) of Natural Gas Imported During the Past 12 Months 01/01/99 - 12/31/99
Long-Term Importer

TRANSCANADA GAS SERVICES INC.

(TransCanada Gas Services)

AVG Daily Quantity

Total Price (Pittsburg, NH)

Commodity Component

Demand Component

Reservation Fee Component

TRANSCANADA GAS SERVICES INC. (1)

(Yankee Gas Services)

AVG Daily Quantity

Total Price (Waddington. NY)

Commodity Component

Demand Component

Reservation Fee Component

\section{TRANSCO ENERGY MARKETING COMPANY (2)}

Canstates Gas Marketing)

AVG Daily Quantity

Commodity Componen

Reservation Fee Component

$\begin{array}{lllllllll}\text { Auth. } & 1999 & 1999 & 1999 & 1999 & 1999 & 1999 & 1999 & 1999\end{array}$

1999

Vols.

Jan.

30.1

$\begin{array}{rrr}0.0 & 0.0 & 2.6 \\ 0.00 & 0.00 & \frac{1.91}{1.91} \\ 0.00 & 0.00 & 1.00 \\ 0.00 & 0.00 & 0.00 \\ 0.00 & 0.00 & 0.00\end{array}$
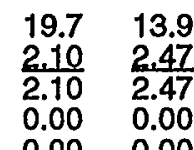

11.9
2.41

000.00

68.5

\begin{tabular}{rrr}
0.0 & 0.0 & 0.0 \\
0.00 & 0.00 & 0.00 \\
\hline 0.00 & 0.00 & 0.00 \\
0.00 & 0.00 & 0.00
\end{tabular}

0.00

0.00

0.00

48.4

\begin{tabular}{llllllllllll}
51.8 & 51.8 & 51.8 & 51.8 & 51.8 & 51.8 & 51.8 & 51.8 & 51.8 & 51.8 & 19.0 & 51.8 \\
2.89 & 2.70 & 2.70 & 2.19 & 2.59 & 2.46 & 2.41 & $\frac{2.44}{2.44}$ & $\frac{2.60}{2.60}$ & $\frac{2.80}{2.80}$ & $\frac{2.81}{2.81}$ & $\frac{2.81}{2.81}$ \\
\hline 2.89 & 2.70 & 2.70 & 2.19 & 2.59 & 2.46 & 2.41 & 2.44 & 2.60 & 2.80 & 0.00 & 0.00 \\
0.00 & 0.00 & 0.00 & 0.00 & 0.00 & 0.00 & 0.00 & 0.00 & 0.00 & 0.00 & 0.00 & 0.00 \\
0.00 & 0.00 & 0.00 & 0.00 & 0.00 & 0.00 & 0.00 & 0.00 & 0.00 & 0.00 & 0.00 & 0.00
\end{tabular}

TRANSCO ENERGY MARKETING COMPANY-BG\&E (3)

AVG Daily Quantity

Total Price (Niagara Falls, NY)

Commodity Component

25.0

15.8
2.68
1.80
0.88
0.00

15.8
2.71
1.83
0.88
0.00

15.8
2.53
1.65
0.88
0.00

0.0
0.00
0.00

$\frac{0.00}{0.00}$

0.00
0.00

0.0
0.00
0.00

$\frac{0.00}{0.00}$

0.00
0.00

$\begin{array}{r}0.0 \\ 0.00 \\ \hline\end{array}$

$\frac{0.00}{0.00}$

0.00
0.00

$\begin{array}{r}0.0 \\ 0.00 \\ \hline\end{array}$

$\frac{0.00}{0.00}$

$\begin{array}{r}0.0 \\ 0.00 \\ \hline\end{array}$

$\frac{0.00}{0.00}$

0.0
0.00
0.00

$\frac{0.00}{0.00}$

0.0
0.00

0.00

0.00

0.0
0.00
0.00
0.00

$\begin{array}{r}0.0 \\ 0.00 \\ \hline 0.00\end{array}$

0.00
Total Price (Niagara Falls. NY)

Demand Component

(Canstates Gas Marketing)

Demand Component

TRANSCO ENERGY MARKETING COMPANY-LILCO (4)

(Canstates Gas Marketing)

AVG Daily Quantity

15.0

$\begin{array}{ll}15.8 & 15 \\ 2.83 & 1.93 \\ 0.90 & 0.9\end{array}$

15.8
$\frac{2.86}{1.96}$
0.90
0.00

15.8
2.68
1.78
0.90
0.00

\begin{tabular}{rr}
0.0 & 0.0 \\
0.00 & -0.00 \\
\hline 0.00 & 0.00 \\
0.00 & 0.00 \\
0.00 & 0.00
\end{tabular}

0.0
0.00
0.00
0.00
0.00

\begin{tabular}{r}
0.0 \\
0.00 \\
\hline 0.00 \\
0.00 \\
0.00
\end{tabular}

\begin{tabular}{r}
0.0 \\
0.00 \\
\hline 0.00 \\
0.00 \\
0.00
\end{tabular}

0.0
0.00
0.00
0.00
0.00

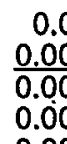

$\begin{array}{rrr}0.0 & 0.0 & 0.0 \\ 0.00 & \frac{0.00}{0.00} & \frac{0.00}{0.00} \\ 0.00 & 0.00 & 0.00 \\ 0.00 & 0.00 & 0.00\end{array}$

4Q99

TOTAL MC

733,224

$3,42,2,506$

$1,531,772$

$1,531,772$

0

$1,423,730$

1) 293,291 was imported at Niagara Falls, NY, and 1,238,481 was imported at Waddinglon, NY. Gas is sold to Yankee Gas Services; replaces Boundary / ANE contracts. Gas resold to Hopewell Cogeneralion Facility and other TEMCO customers.

(4) This contract has been terminated. 
Page - 49

\section{VOLUME AND PRICE REPORT}

\section{Long-Term Imports}

Volumes (MMcf/d) \& Prices (\$MMBTU) of Natural

Gas Imported During the Past 12 Months 01/01/99 - 12/31/99

Long-Term Importer

$\begin{array}{lllllllllllll}\text { Auth. } & 1999 & 1999 & 1999 & 1999 & 1999 & 1999 & 1999 & 1999 & 1999 & 1999 & 1999 & 1999\end{array}$

Vols. Jan. Eeb. Mar Apr May Jun Jul Aug. Sep Oct Nov Dec.

$4 Q 99$

TOTALMCI

TRANSCO ENERGY MARKETING COMPANY-PSE\&G (1)

(Canstates Gas Marketing)

AVG Daily Quantity

Total Price (Niagara Falls NY)

Commodity Component

Reservation Fee Component
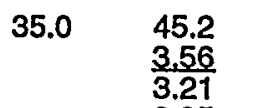

45.2
3.56
3.21
0.35

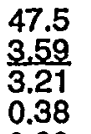

54.1

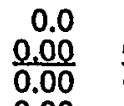

0.00

0.00

0.00

\begin{tabular}{ll}
13.5 & 16.8 \\
2.30 & 2.22 \\
\hline 1.67 & 1.59 \\
0.63 & 0.63
\end{tabular}

$\begin{array}{ll}0.63 & 0.63 \\ 0.00 & 0.00\end{array}$

16.5
2.20
1.57
0.63
0.00

16.6
2.28
1.65
0.63

$0.00 \quad 0.00$

12.2
2.16
1.91
0.25
0.00

Reservation Fee Component

0

UTILICORP UNITED, INC. (2)

(ProGas Limited)

AVG Daily Quantity

Total Price (Noyes, Minnesota)

Commodity Componen

Demand Component

Reservation Fee Component

UTILICORP UNITED, INC. (3)

(TCGS Ltd.)

AVG Daily Quantity

Total Price (Noyes. Minnesota)

Commodity Component

Demand Component

Reservation Fee Component
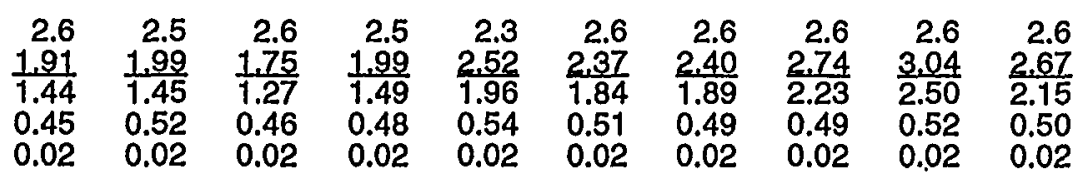

\begin{tabular}{r}
2.6 \\
3.20 \\
\hline 2.66 \\
0.52 \\
0.02
\end{tabular}

2.6
2.27
1.77
0.48
0.02

238,046

YTD

TOTALMCf
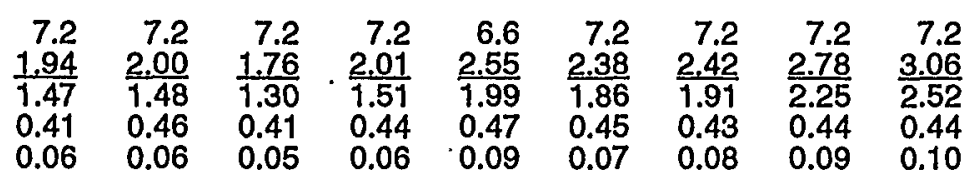

\begin{tabular}{r}
7.2 \\
2.68 \\
\hline 2.17 \\
0.42 \\
0.09
\end{tabular}

$\begin{array}{r}7.2 \\ 3.20 \\ \hline\end{array}$

$\frac{3.20}{2.68}$

7.2
2.27
1.79
0.41

663,596

$2,611,951$

(1) This contract has been terminated.

(2) Volumes used by Michlgan Gas Utilities 


\section{VOLUME AND PRICE REPORT}

\section{Long-Term Imports}

Volumes (MMcf/d) \& Prices (\$MMBTU) of Natural

Gas Imported During the Past 12 Months 01/01/99 - 12/31/99

Long-Term Importer

Auth. $\quad \begin{array}{llll}1999 & 1999 & 1999 & 1999\end{array}$

Vols.

Jan.

1.0

\begin{tabular}{rrr}
1.0 & 1.0 & 1.0 \\
1.99 & $\frac{1.93}{1.22}$ & $\frac{1.88}{1.15}$ \\
\hline 1.26 & 0.71 & 0.73 \\
0.73 & 0.71 & 0.00 \\
0.00 & 0.00 & 0.00
\end{tabular}

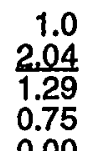

1.0
2.51
1.76
0.75
0.00

1.0
2.39
1.60
0.79
0.00

1.0
2.46
1.68
0.78
0.00

1.0
2.76
1.98
0.78
0.00

1.0
3.15
2.36
0.79
0.00

1.0
2.80
2.01 $\frac{2.80}{2.01}$
0.79
0.00

$\frac{3.22}{2.43}$

2.36

Commodity Componen

Reservation Fee Component

VERMONT GAS SYSTEMS INC. (1)

(TCGS Ltd.)

AVG Daily Quantity

Total Price (Highgate Spr. VT)

Commodity Component

Demand Component

Reservation Feo Component

\section{VERMONT GAS SYSTEMS, INC.}

(Renaissance Energy Ltd.)

AVG Daily Quantity

Total Price (Hiahgate Spr. VT)

Commodity Componen

Demand Component

Reservation Fee Component

VERMONT GAS SYSTEMS, INC. (2)

(Various Suppliers)

AVG Daily Quantity

Total Price (Highgate Spr. VT)

Commodity Component

Demand Component

Reservation Fee Component

11.0

11.3
$\frac{1.95}{1.13}$
0.82

10.9
2.05
1.14
0.91

11.0

9.8
2.28
1.28

0.00

0.91

8.0

\begin{tabular}{rrr}
8.8 & 8.7 & 8.0 \\
2.42 & 2.35 & 2.43 \\
\hline 1.84 & 1.69 & 1.78 \\
0.58 & 0.66 & 0.65 \\
0.00 & 0.00 & 0.00
\end{tabular}

8.0
2.53
1.86
0.67
0.00

7.3
2.88
2.16
0.72
0.00

2.5
4.52
2.21
2.31
0.00

1.8
$\frac{5.20}{2.14}$
3.06
0.00

1.8
$\frac{5.17}{2.15}$
3.02
0.00

5.4
$\frac{3.72}{2.66}$
1.06

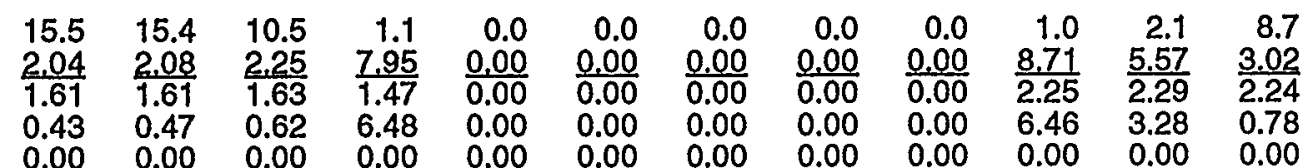

WISCONSIN ELECTRIC POWER CO. (GAS OPS)

(Progas Limited)

AVG Daily Quantity

Total Price (Noyes. Minnesota)

Commodity Componen

Demand Component

Reservation Fee Component
10.7

$\begin{array}{lllllll}10.7 & 10.7 & 10.7 & 10.7 & 9.3 & 10.7 & 10.7 \\ \frac{1.92}{1.44} & \frac{1.99}{1.45} & \frac{1.75}{1.27} & \frac{1.99}{1.49} & \frac{2.56}{1.96} & \frac{2.37}{1.84} & \frac{2.41}{1.89} \\ 0.44 & 0.50 & 0.44 & 0.46 & 0.53 & 0.47 & 0.46 \\ 0.04 & 0.04 & 0.04 & 0.04 & 0.07 & 0.06 & 0.06\end{array}$

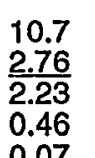

\begin{tabular}{l}
10.7 \\
2.76 \\
\hline 2.23 \\
0.46 \\
0.07
\end{tabular}
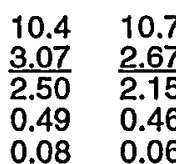

10.7
2.67
2.15
0.46
0.06

4.3
$\frac{3.96}{2.66}$
1.20

10.7
2.26
1.77
0.46
0.03
$4 Q 99$

TOTALMCI

YTD

92,000

365,000

888,009

$3,077,346$

735,894

$2,314,308$

363,418

$1,637,050$

794,127

$3,671,007$

(1) Daily contract demand was reduced from $32 \mathrm{MMcf}$ to $21 \mathrm{MMcf}$, effective April 1, 1995. On November 1, 1998, the daily contract demand was reduced to 11 MMct.
(2) This import is part of a gas services agreement with Gaz Metropolitain Limited Partnership. In October 1998. Vermont imported 1,010 Mcf at a price of $\$ 220.17$. 


\section{VOLUME AND PRICE REPORT}

\section{Long-Term Imports}

Volumes (MMcf/d) \& Prices (\$MMBTU) of Natural

Gas Imported During the Past 12 Months 01/01/99 - 12/31/99

Long-Term Importer

Auth. $\quad 1999 \quad 1999 \quad 1999 \quad 1999$

WISCONSIN ELECTRIC POWER CO. (GAS OPS)

(TCGS Ltd.)

AVG Daily Quantity

Total Price (Noyes. Minnesota)

Commodity Component

Demand Component

Reservation Fee Component

WISCONSIN FUEL \& LIGHT COMPANY

(Progas Limited)

AVG Daily Quantity

Total Price (Noyes. Minnesota)

Commodity Componen

Demand Component

Reservation Fee Component

WISCONSIN FUEL \& LIGHT COMPANY

(TCGS Ltd.)

AVG Daily Quantity

Total Price (Noves. Minnesota)

Commodity Component

Demand Component

Reservation Fee Component

WISCONSIN GAS COMPANY (1)

(ProGas Limited (I))

AVG Daily Quantity

Commodity Component

Demand Component

Reservation Fee Component

WISCONSIN GAS COMPANY

(ProGas Limited (II))

AVG Daily Quantity

Commodity Component

Demand Component

Reservation Fee Component

(1) Imports Include speclal spot purchases from ProGas.

26.5

\begin{tabular}{llll}
26.4 & 26.4 & 26.4 & 26.6 \\
1.99 & 2.06 & 1.83 & 2.05 \\
\hline 1.51 & 1.52 & 1.36 & 1.55 \\
0.43 & 0.49 & 0.43 & 0.45
\end{tabular}

23.2
2.54
1.96
0.51
0.07

26.6
2.36
1.85
0.46
0.05

26.6
2.39
1.89
0.44
0.06

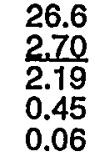

.6
.70
.19
.45

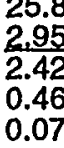

.95

26.6
2.61
2.12
0.43

0.43
0.06

3.0

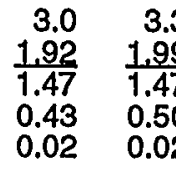

7.4

$\begin{array}{rrr}4.3 & 5.3 & 7.1 \\ 2.35 & 2.27 & 1.88 \\ 1.57 & 1.56 & 1.41 \\ 0.70 & 0.65 & 0.43 \\ 0.08 & 0.06 & 0.04\end{array}$

6.6

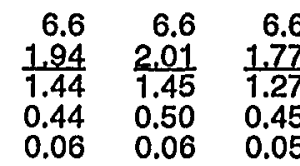

28.5

\begin{tabular}{lll}
28.9 & 28.9 & 28.9 \\
1.93 & 2.01 & 1.76 \\
\hline 1.44 & 1.45 & 1.27 \\
0.43 & 0.50 & 0.44 \\
0.06 & 0.06 & 0.05
\end{tabular}

0.06

$\begin{array}{rr}3.0 & 3.0 \\ .76 & 1.98 \\ .30 & 1.50 \\ 0.44 & 0.46 \\ 0.02 & 0.02 \\ . & \\ & . \\ 7.1 & 7.4 \\ 1.88 & 2.07 \\ 1.41 & 1.58 \\ 0.43 & 0.44 \\ 0.04 & 0.05\end{array}$
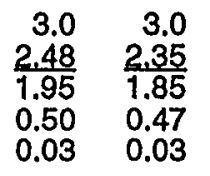

3.0
2.39
1.90
0.46
0.03

3.0
2.69
2.20
0.46

3.0

$\frac{3,00}{2.48}$

0.48
0.04

$\begin{array}{r}3.0 \\ 2.65 \\ \hline\end{array}$

$\frac{2.65}{2.16}$

0.46
0.03

3.0
3.18

3.18
2.66
0.48

0.48
0.04

2.31

.82

0.46
0.03

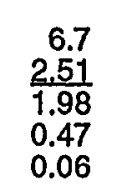

7.4
2.40
1.89
0.45
0.06

7.4
2.42
1.94
0.42
0.06

7.4
2.71
2.21
0.43
0.07

7.4
2.96
2.46
0.43
0.07

7.4
2.66
2.18

$\begin{array}{r}3.7 \\ 3.58 \\ \hline 255\end{array}$

2.55
0.87

7.3
2.35
1.88

1.88

0.41
0.06

6.7
2.02
1.49
0.47
0.06

6.2
2.53
1.96
0.49
0.08

6.7
2.39
1.84
0.48

6.7
2.41
1.89
0.46
0.06

0.48

6.7
2.78
2.23
0.46
0.09

$\begin{array}{r}2.0 \\ 4.43 \\ \hline 2.50\end{array}$

2.50

1.60

2.2
4.21
2.66

2.66
0.44

6.2
2.01
1.45
0.50

6.7
2.27
1.77
0.46
0.04

\begin{tabular}{ll}
28.9 & 26.1 \\
2.01 & 2.55 \\
\hline 1.49 & 1.96 \\
0.46 & 0.50 \\
0.06 & 0.09
\end{tabular}

28.9
2.39
1.84
0.48
0.07

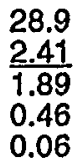

28.9
2.41
1.89
0.46
0.06

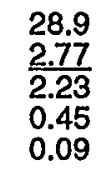

8.7
4.41
2.50
1.58

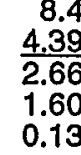

26.9

$\frac{2.01}{1.45}$

1.45
0.50

0.06

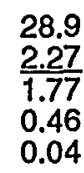

568,305

$2,409,00$

458,137

$2,117,177$

4099

TOTAL MCF

YTD

$2,025,326 \quad 9,145,425$

275,540

$1,101,160$

$9,161,725$ 
VOLUME AND PRICE REPORT

\section{Long-Term Imports}

Volumes (MMct/d) \& Prices (\$MMBTU) of Natural

Gas Imported During the Past 12 Months 01/01/99 - 12/31/99

Long-Term Importer

Auth. 1999

WISCONSIN GAS COMPANY (1)

(TCGS Ltd.)

AVG Daily Quantity

Total Price (Noyes. Minnesota)

Commodity Component

Demand Component

Reservation Fee Component

WISCONSIN GAS COMPANY (2)

(TCGS Ltd.)

AVG Daily Quantity

Total Price (Noyes, Minnesota)

Commodity Component

Demand Component

Reservation Fee Component

WISCONSIN POWER \& LIGHT COMPANY

(ProGas Limited)

AVG Daily Quantity

Total Price (Noyes. Minnesota)

Commodity Component

Demand Component

Reservation Fee Component

WISCONSIN POWER \& LIGHT COMPANY

(TCGS Ltd.)

AVG Daily Quantity

Total Price (Noyes. Minnesota)

Commodity Componen

Demand Component

Reservation Fee Component

WISCONSIN PUBLIC SERVICE CORPORATION

(ProGas Limited)

AVG Daily Quantity

Total Price (Noyes, Minnesota)

Commodity Component

Demand Component

Reservation Fee Component
89.4

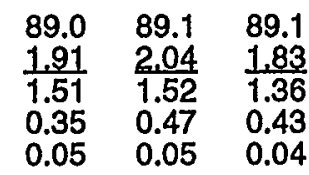

1.9

1.9
2.04
1.55
0.44
0.05

2.0
$\frac{2.43}{1.96}$
$\frac{0}{2.41}$

0.41

2.0

1.85

0.04

$\begin{array}{rrr}1.9 & 1.9 & 1.9 \\ 1.92 & 2.04 & 1.82 \\ 1.51 & 1.52 & 1.36 \\ 0.37 & 0.48 & 0.42 \\ 0.04 & 0.04 & 0.04\end{array}$

3.4

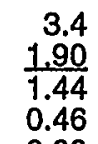

\begin{tabular}{r}
3.4 \\
1.90 \\
\hline 1.44 \\
0.46 \\
0.00
\end{tabular}

\begin{tabular}{r}
3.4 \\
1.97 \\
\hline 1.45 \\
0.52 \\
0.00
\end{tabular}

\begin{tabular}{r}
3.4 \\
1.73 \\
\hline 1.27 \\
0.46 \\
0.00
\end{tabular}

\begin{tabular}{r}
3.4 \\
1.97 \\
\hline 1.49 \\
0.48 \\
0.00
\end{tabular}

3.0
2.50
1.96
0.54
0.00

0.54
0.00

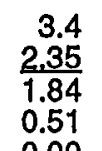

3.4
2.38
1.89

2.38
1.89
0.49

$\begin{array}{ll}1.51 & 0.49 \\ 0.00 & 0.00\end{array}$

2.0
2.38
1.89
0.43

1.9
2.69
2.19
0.44
0.06

0.8
$\frac{3.71}{2.42}$

20.9
2.60

1.11

$\frac{2.60}{2.12}$

0.42

6.3
4.26
2.56
1.45
0.25

1.9
2.26
1.79
0.42
0.05

895,601

$1,390,137$

8.4

$\begin{array}{rrr}8.3 & 8.3 & 8 \\ 1.98 & 2.04 & 1.83 \\ 1.51 & 1.52 & 1.36 \\ 0.47 & 0.52 & 0.47 \\ 0.00 & 0.00 & 0.00\end{array}$

\begin{tabular}{rr}
8.3 & 8.4 \\
1.83 & 2.05 \\
\hline 1.36 & 1.55 \\
0.47 & 0.50
\end{tabular}

$\begin{array}{ll}7.5 & 8.3\end{array}$

$\frac{2.52}{1.96} \quad \frac{2.37}{1.85}$

1.96
0.56
0.00

1.85
0.52

8.3

$\frac{2.39}{1.89}$

1.89
0.50

0.00

3.4
2.72
2.23
0.49

3.4

$\frac{3.02}{2.50}$

0.52

3.4
2.65
2.15

2.15
0.50

$\begin{array}{r}3.4 \\ 3.18 \\ \hline 2.66\end{array}$

2.66

0.00

0.00

$\begin{array}{lll}.50 & 0.52 & 0.49 \\ .00 & 0.00 & 0.00\end{array}$

$\begin{array}{lrll}11.1 & 5.9 & 11.1 & 11.1 \\ \frac{1.92}{1.44} & \frac{2.47}{1.45} & \frac{1.75}{1.27} & \frac{2.00}{1.49} \\ 0.43 & 0.93 & 0.44 & 0.46 \\ 0.05 & 0.09 & 0.04 & 0.05\end{array}$

10.0
2.54
1.96
0.50
0.08

11.1
2.38
1.84
0.48
0.06

11.1
2.42
1.89
0.46
0.07

8.3
2.71
2.19
0.52

5.5
3,22
2,42

8.3
2.62
2.12

2.12

2.8
4.14
2.56

8.3
$\frac{2.27}{1.79}$

0.48

$\begin{array}{lllll}0.00 & 0.00 & 0.00 & 0.00 & 0.00\end{array}$

601,041

$2,771,054$

(1) Imports include special spot purchases from TransCanada.
(2) Imports include special spot purchases from TransCanada. 
VOLUME AND PRICE REPORT

\section{Long-Term Imports}

Volumes (MMcf/d) \& Prices (\$MMBTU) of Natural

Gas Imported During the Past 12 Months 01/01/99 - 12/31/99

Lona-Term Importer

Auth.

1999

1999

1999

1999

1999

1999

1999

1999

WISCONSIN PUBLIC SERVICE CORPORATION

(TCGS Ltd.)

AVG Daily Quantity

Total Price (Noves. Minnesota)

Commodity Component

Demand Component

Reservation Fe日 Component

27.4

$\begin{array}{llll}27.3 & 10.7 & 27.3 & 27.6\end{array}$

$\begin{array}{lll}1.98 & 2.87 & 1.83\end{array}$

$1.51 \quad 1.52$

$\begin{array}{ll}0.04 & 0.11\end{array}$

1.36
0.43

0.04

27.6
$\frac{2.05}{1.55}$
0.45

24.9

$\frac{2.51}{1.96}$

$\frac{2.36}{1.85}$

\begin{tabular}{ll}
27.6 \\
6 & 2.39 \\
\hline 5 & 1.89 \\
05 & 0.44 \\
\hline 5 & 0.06
\end{tabular}

$\begin{array}{lll}27.6 & 17.3 & 27.6\end{array}$

$\frac{2.70}{2.19}$

$\begin{array}{llllll}0.49 & 0.46 & 0.44 & 0.45 & 0.71 & 0.43 \\ 0.06 & 0.05 & 0.06 & 0.06 & 0.11 & 0.06\end{array}$

$\begin{array}{llllll}0.49 & 0.46 & 0.44 & 0.45 & 0.71 & 0.43 \\ 0.06 & 0.05 & 0.06 & 0.06 & 0.11 & 0.06\end{array}$

$\frac{3.24}{2.42}$

$\frac{2.61}{2.12}$

$\begin{array}{r}17.3 \\ 3.39 \\ \hline\end{array}$

$\frac{3.39}{2.56}$

$\frac{3.39}{2.56}$

$0.71 \quad 0.42$

YANKEE GAS SERVICES CO

(Alberta NE Gas (AEC Oil \& Gas Company))

AVG Daily Quantity

Total Price (Waddington, NY)

Commodity Componen

Demand Component

Reservation $\mathrm{Fee}$ Component

3.9

\begin{tabular}{rr}
3.9 & 3.9 \\
1.90 & 1.95 \\
\hline 1.09 & 1.12 \\
0.81 & 0.83 \\
0.00 & 0.00
\end{tabular}

3.9
1.80
0.98
0.82
0.00

3.9
2.03
1.19
0.84
0.00

3.9
2.52
1.67
0.85
0.00

$\frac{3.9}{2.47}$

3.9
2.48
1.61
0.87
0.00

3.9
2.82
1.95
0.87
0.00

\begin{tabular}{r}
3.9 \\
3.11 \\
\hline 2.23 \\
0.88 \\
0.00
\end{tabular}

3.9
2.94
2.06
0.88

3.9
$\frac{3.34}{2.46}$

0.00

28.7

$\begin{array}{ll}27.6 & 28.7 \\ 1.97 & 1.93 \\ 1.24 & 1.17 \\ 0.73 & 0.76 \\ 0.00 & 0.00\end{array}$

\section{7}

28.7

AVG Daily Quantity

Total Price (Waddington. NY)

Demand Component

Reservation Fee Component

YANKEE GAS SERVICES CO. (2)

(Alberta NE Gas (TCGS Ltd. 2))

AVG Daily Quantity

Total Price (Waddington. NY)

Commodity Component

Demand Component

Reservation Fee Component

YANKEE GAS SERVICES CO.

(Alberta Northeast Gas (ProGas))

AVG Daily Quantity

Total Price (Waddington, NY)

Commodity Component

Demand Component

Reservation Fee Component

11.3

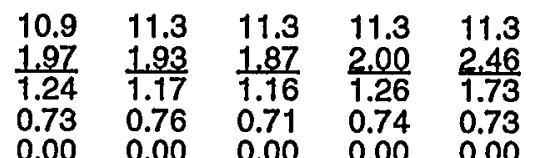

$0.00 \quad 0.00$

0.00

28.7
2.46
1.73
0.73
0.00

28.7
2.36
1.57
0.79
0.00

28.7
2.40
1.65
0.75
0.00

28.7
2.70
1.95
0.75
0.00

28.7
$\frac{3.10}{2.32}$
0.78
0.00

28.7
2.73
1.97
0.76

$\frac{28.7}{2.39}$

0.79

0.00

3.9
2.67
1.79
0.88
0.00

361,376

$1,433,720$

0.0
0.00

$\frac{0.00}{0.00}$

0.00

0.00

$1,748,555$

$\begin{array}{ll}11.3 & 11.3 \\ 2.36 & 2.40 \\ 1.57 & 1.65 \\ 0.79 & 0.75\end{array}$

11.3
2.70
1.95
0.75

11.3
3.10
2.32
0.78
0.00

11.3
2.73
1.97
0.76
0.00

$\begin{array}{ll}11.3 & 0.0\end{array}$

$\frac{3.18}{2.39}$

2.39

0.79
0.00

0.00
0.00
0.00

0.00
0.00

9.9

\begin{tabular}{rr}
9.9 & 9.9 \\
1.92 & 1.96 \\
\hline 1.06 & 1.08 \\
0.86 & 0.88
\end{tabular}

9.9
1.80
0.93
0.87
0.00

\begin{tabular}{rr}
9.9 & 9.9 \\
2.10 & 2.53 \\
\hline 1.25 & 1.63 \\
0.85 & 0.90 \\
0.00 & 0.00
\end{tabular}

9.9
2.45
1.52
0.93
0.00

9.9
2.51
1.60
0.91
0.00

9.9
2.88
1.97
0.91
0.00

\begin{tabular}{r}
9.9 \\
3.16 \\
\hline 2.24 \\
0.92 \\
0.00
\end{tabular}

$\begin{array}{r}9.9 \\ 2.99 \\ \hline\end{array}$

$\frac{2.99}{2.07}$

5.2

$\frac{3.38}{2.48}$

9.9

$\frac{2.79}{1.87}$

$\begin{array}{lll}0.92 & 0.90 & 0.92\end{array}$

$\begin{array}{lllllllllllll}0.00 & 0.00 & 0.00 & 0.00 & 0.00 & 0.00 & 0.00 & 0.00 & 0.00 & 0.00 & 0.00 & 0.00\end{array}$

(1) Beginning on 12/1/99, these volumes will be Imported and reported under TransCanada Gas Services' long-term authorizallon in Order Number 1543.
(2) Beginning on 1211/99, these volumes will be Imported and reported under TransGanada Gas Services' long-term authorizallon in Order Number 1543. 


\section{VOLUME AND PRICE REPORT}

\section{Long-Term Imports}

Volumes (MMct/d) \& Prices (\$MMBTU) of Natural

Gas Imported During the Past 12 Months 01/01/99 - 12/31/99

Leng-Term importer

YANKEE GAS SERVICES CO.

(Alberta Northeast Gas (Producers Marketing))

AVG Daily Quantity

Total Price (Waddington. NY)

Commodity Componen

Demand Component

Reservation Fee Component

Auth. $\quad 1999 \quad 1999 \quad 1999 \quad 1999$

1999

19991999

1999

1999

Vols.

Jan.

Mar.

5.2

\begin{tabular}{rr}
5.2 & \\
1.92 & 1 \\
\hline 1.08 & 1 \\
0.84 & 0 \\
0.00 & 0.00
\end{tabular}

$5.2 \quad 5.1$

5.1
1.80
0.96
0.84

5.2
2.10
1.25
0.85

5.2
2.53
1.66
0.87
0.00

0.85

0.84
0.00

0.00
5.2
2.45

2.51

2.51
1.63
0.88
0.00

5.2
2.88
2.00

2.00

$\begin{array}{ll}0.88 & 0.89 \\ 0.00 & 0.00\end{array}$

0.00
$2.88 \quad 3.16$

Total Mcf 


\section{VOLUME AND PRICE REPORT}

\section{Long Term Exports}

Volumes (MMcf/d) \& Prices (\$/MMBTU) of Natura

Gas Exported During the Past 12 Months 01/01/99 - 12/31/99

Long-Term Exporter

MARATHON OIL COMPANY

(Tokyo Electric Co./Tokyo Gas Co.)

Auth.

$1999 \quad 1099$

1999

Jan. Fob. Mar. 1999

$1999 \quad 1999$

1999

$\begin{array}{lllll}1999 & 1999 & 1999 & 1999 & 1999\end{array}$

May.

54.7
2.85
2.85
0.00
0.00

54.5
3.08
3.08
0.00
0.00

56.3
3.20
3.20
0.00
0.00

AVG Daily Quantity

Total Price (Kenai. Alaska)

Commodity Component

Demand Component

Reservation Fee Component

PHILLIPS ALASKA NATURAL GAS CORPORATION

(Tokyo Electric Co./Tokyo Gas Co.)

AVG Daily Quantity

Total Price (Kenai. Alaska)

Commodity Component

Demand Component

Reservation Fee Component

136.4

50.4

$\begin{array}{lll}55.9 & 59.4 & 52.1 \\ 2.92 & 2.91 & 2.85 \\ \frac{2.92}{2.91} & 2.85 \\ 0.00 & 0.00 & 0.00 \\ 0.00 & 0.00 & 0.00\end{array}$

56.9
2.74
2.74
0.00

54.6
2.75
2.75
0.00
0.00

37.4
2.74
2.74

0.00

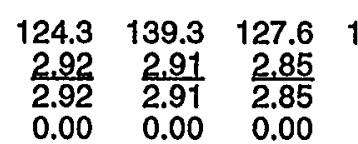

132.7
2.74
2.74
0.00

0.00
.

\begin{tabular}{ll}
56.0 & 54.1 \\
3.52 & 3.77 \\
\hline 3.52 & 3.77 \\
0.00 & 0.00 \\
0.00 & 0.00
\end{tabular}

$0.00 \quad 0.00$

Total Mcf

\begin{tabular}{|c|c|c|c|c|c|}
\hline $\begin{array}{l}87.8 \\
2.74 \\
2.74 \\
0.00 \\
0.00\end{array}$ & $\begin{array}{r}128.3 \\
2.85 \\
2.85 \\
0.00 \\
0.00\end{array}$ & $\begin{array}{r}127.5 \\
3.08 \\
3.08 \\
0.00 \\
0.00\end{array}$ & $\begin{array}{r}130.6 \\
3.20 \\
3.20 \\
0.00 \\
0.00\end{array}$ & $\begin{array}{l}83.2 \\
3.25 \\
3.25 \\
0.00 \\
0.00\end{array}$ & $\begin{array}{r}130.0 \\
3.52 \\
3.52 \\
0.00 \\
0.00\end{array}$ \\
\hline
\end{tabular}

4Q999

TOTALMCF

$4,500,644$

$19,108,230$

\section{$10,379,064 \quad 44,499,314$}

$14,879,708$

$63,607,544$ 


\section{SHORT-TERM BLANKET IMPORTS}

\section{Year \&}

Month

Active

Importers
Est. Volumes

(MMCF)
Weighted Avg.

Price (\$/MMBTU)

1997

$\begin{array}{ll}\text { January } & 98 \\ \text { February } & 99 \\ \text { March } & 95 \\ \text { April } & 87 \\ \text { May } & 88 \\ \text { June } & 88 \\ \text { July } & 88 \\ \text { August } & 80 \\ \text { September } & 80 \\ \text { October } & 83 \\ \text { November } & 90 \\ \text { December } & 85\end{array}$

144857

2.97

123770

2.17

133195

121258

120037

114877

119335

123057

126596

137702

149872

133682

1.43

1.38

1.55

1.55

1.50

1.47

1.65

1.94

2.38

1.86

1998

January

89

156808

1.81

February

132208

1.66

138012

1.72

April

135700

1.79

May

133217

1.74

128632

1.63

July

151492

163301

152042

1.72

1.63

1.51

September

149495

1.78

October

138962

1.99

December

153651

2.01

1999

$\begin{array}{ll}\text { January } & 88 \\ \text { February } & 87 \\ \text { March } & 84 \\ \text { April } & 75 \\ \text { May } & 76 \\ \text { June } & 76 \\ \text { July } & 79 \\ \text { August } & 80 \\ \text { September } & 79 \\ \text { October } & 80 \\ \text { November } & 86 \\ \text { December } & 86\end{array}$

172928

1.89

162118

166059

148628

165456

153682

178870

197471

191807

180089

189859

186244

1.77

1.64

1.71

2.05

2.01

2.04

2.26

2.50

2.41

2.74

2.26 


\section{SHORT-TERM IMPORTERS \\ Estimated Volumes (MMCF)}

AEC MARKETING (USA) INC.

AEC WEST LTD.

ALCOA, INC.

AMERICAN HUNTER EXPLORATION LTD.

AMOCO CANADA MARKETING CORPORATION

AMOCO ENERGY TRADING CORPORATION

ANDROSCOGGIN ENERGY LLC

AQUILA ENERGY MARKETING CORPORATION

ARCO PRODUCTS COMPANY .

AVISTA CORPORATION

AVISTA ENERGY, INC.

BARRINGTON PETROLEUM LTD.

BC GAS UTILITY LTD.

BIG SKY GAS MARKETING

CABOT OIL \& GAS MARKETING CORPORATION

CANWEST GAS SUPPLY U.S.A., INC.

CARTHAGE ENERGY SERVICES, INC.

CASCADE NATURAL GAS CORPORATION

CHEVRON U.S.A. INC.

CINERGY MARKETING \& TRADING, LLC

CMS MARKETING SERVICES AND TRADING COMPANY (1)

COAST ENERGY GROUP

COENEAGY TRADING COMPANY

COGEN ENERGY TECHNOLOGY, L.P.

COMMONWEALTH GAS COMPANY

CONSUMERS ENERGY COMPANY

CORAL ENERGY RESOURCES, L.P.

CORAL ENERGY RESOURCES, L.P. (LNG)

CRESTAR ENERGY MARKETING CORPORATION

CXY ENERGY MARKETING (U.S.A.) INC.

DARTMOUTH POWER ASSOCIATES L.P.

DEK ENERGY COMPANY

DISTRIGAS CORPORATION (2)

DUKE ENERGY LNG MKTG \& MGMT CO (3)

DUKE ENERGY LNG SALES, INC. (4)

DUKE ENERGY TRADING AND MKTG SERVICES L.LC.

DYNEGY MARKETING \& TAADE

EL PASO ENERGY MARKETING COMPANY

ENERGY WEST RESOURCES, INC.

ENGAGE ENERGY CANADA, L.P.

ENGAGE ENERGY U.S., L.P.

ENRON CAPITAL \& TRADE RESOURCES CORP.

ENRON ENERGY SERVICES, INC.

ENRON GAS LIQUIDS, INC.

ENRON INTERNATIONAL GAS SALES COMPANY

ERI SERVICES, INC.

FLORIDA POWER \& LIGHT COMPANY

\begin{tabular}{|c|c|c|c|c|}
\hline $\begin{array}{c}1998 \\
\text { Oct-Dec. }\end{array}$ & $\begin{array}{c}1999 \\
\text { Jan.-Mar. }\end{array}$ & $\begin{array}{c}1999 \\
\text { Apr-Jun. }\end{array}$ & $\begin{array}{c}1999 \\
\text { Jul.-Sep. }\end{array}$ & $\begin{array}{c}1999 \\
\text { Oct.-Dec. }\end{array}$ \\
\hline 6271 & 6143 & 6146 & 6581 & 6346 \\
\hline 1360 & 7226 & 7454 & 7406 & 7378 \\
\hline 651 & 891 & 1490 & 1286 & 1516 \\
\hline 593 & 0 & 0 & 0 & 0 \\
\hline 14173 & 10014 & 10157 & 10099 & 13086 \\
\hline 127 & 497 & 0 & 0 & 0 \\
\hline 0 & 0 & 0 & 0 & 167 \\
\hline 1426 & 3273 & 247 & 5340 & 10701 \\
\hline 1310 & 1790 & 2283 & 2351 & 2335 \\
\hline 4089 & 5479 & 3920 & 3365 & 6137 \\
\hline 11711 & 13961 & 17776 & 11407 & 12689 \\
\hline 0 & 0 & 0 & 147 & 451 \\
\hline 0 & 0 & 85 & 127 & 896 \\
\hline 751 & 774 & 817 & 762 & 991 \\
\hline 1008 & 1238 & 326 & 538 & 507 \\
\hline 3660 & 2244 & 3062 & 1406 & 2028 \\
\hline 1582 & 1533 & 1262 & 1549 & 1462 \\
\hline 2245 & 3351 & 542 & 40 & .3596 \\
\hline 120 & 139 & 328 & 144 & 127 \\
\hline 3 & 3 & 0 & 0 & 0 \\
\hline 0 & 0 & 2076 & 6864 & 6949 \\
\hline 0 & 1352 & 1354 & 1752 & 2309 \\
\hline 167 & 295 & 31 & 177 & 171 \\
\hline 510 & 1126 & 787 & 0 & 0 \\
\hline 0 & 0 & 0 & 0 & 276 \\
\hline 1048 & 0 & 0 & 0 & 0 \\
\hline 4566 & 2215 & 4031 & 5121 & 6456 \\
\hline 0 & 0 & 0 & 2576 & 0 \\
\hline 196 & 10 & 795 & 306 & 142 \\
\hline 10550 & 10803 & 7808 & 14051 & 15079 \\
\hline 96 & 54 & 42 & 302 & 384 \\
\hline 815 & 1009 & 903 & 1056 & 1062 \\
\hline 2039 & 2433 & 1356 & 5860 & 5874 \\
\hline 5967 & 3047 & 3638 & 5676 & 0 \\
\hline 3 & 1306 & 1853 & 48 & 1651 \\
\hline 21471 & 17155 & 27204 & 46403 & 45460 \\
\hline 1735 & 4100 & 3460 & 5981 & 1982 \\
\hline 159 & 1380 & 1113 & 643 & 1392 \\
\hline 657 & 628 & 730 & 508 & 446 \\
\hline 10938 & 13852 & 6577 & 6792 & 6675 \\
\hline 12964 & 17181 & 16834 & 18792 & 22861 \\
\hline 23850 & 27929 & 30041 & 27654 & 24844 \\
\hline 0 & 0 & 0 & 0 & 904 \\
\hline 2585 & 0 & 0 & 0 & 0 \\
\hline 0 & 0 & 0 & 0 & 2713 \\
\hline 3779 & 0 & 0 & 0 & 0 \\
\hline 624 & 878 & 0 & 0 & 0 \\
\hline
\end{tabular}

(1) Sales for resale rather than imports. Actual imports: 2nd Qtr $99(2,314)$; 3rd Qtr $99(9,677)$; 4th Qtr $99(9,496)$.

(2) Actual imports: 4th Qtr 98 (2,353); 1st Qtr $99(2,557)$; 2nd Qtr $99(1,461)$; 3rd Qtr $99(5,860)$; 4 th Qtr $99(5,874)$.

(3) Actual imports: 4th Qtr $98(5,015)$; 1st Qtr $99(2,647)$; 2nd Qtr $99(4,910)$; 3rd Qtr $99(5,162)$; 4th Qtr $99(0)$.

(4) Actual imports: 4th Qtr 98 (575); 1st Qtr $99(1,014)$; 2nd Qtr 99 (813); 3rd Qtr 99 (456); 4th Qtr $99(2,671)$. 
Page - 58

\section{SHORT-TERM IMPORTERS}

Estimated Volumes (MMCF)

H.Q. ENERGY SERVICES (U.S.) INC.

HESS ENERGY SERVICES COMPANY, LLC

HOWARD ENERGY MARKETING, INC.

HUSKY GAS MARKETING, INC.

IDACORP, ENERGY SOLUTIONS L.P. (1)

IGI RESOURCES, INC.

INDECK-OSWEGO, LP.

INDECK-YERKES, LP.

INTERENERGY CORPORATION

KN MARKETING, L.P.

KCS ENERGY MARKETING, INC.

KOCH ENERGY TRADING, INC.

MASSPOWER

MONTANA-DAKOTA UTILITIES CO.

MURPHY GAS GATHERING

NATIONAL FUEL GAS DISTRIBUTION

NATIONAL FUEL RESOURCES, INC.

NATIONAL STEEL CORPORATION

NEW YORK STATE ELECTRIC \& GAS CORPORATION

NORTH AMERICAN ENERGY, INC.

NORTHEAST GAS MARKETS LLC.

NORTHERN STATES POWER COMPANY (WI)

NORTHWEST NATURAL GAS COMPANY

NUI ENERGY BROKERS, INC.

NUMAC ENERGY (U.S.) INC.

ONYX GAS MARKETING COMPANY, LC.

PAN-ALBERTA GAS (U.S.), INC.

PANCANADIAN PETROLEUM COMPANY

PAWTUCKET POWER ASSOCIATES L.P.

PEMEX GAS

PETRO-CANADA HYDROCARBONS, INC.

PG\&E CORE PROCUREMENT DEPT.

PHIBRO INC.

PITTSFIELD GENERATING PLANT, LP.

POCO MARKETING LTD.

PORTLAND GENERAL ELECTRIC COMPANY

PORTLAND NATURAL GAS TRANSMISSION SYSTEM

PRAIRIELANDS ENERGY MARKETING, INC.

PREMSTAR ENERGY CANADA, LTD.

PROGAS U.S.A., INC.

PUGET SOUND ENERGY, INC.

QUESTAR ENERGY TRADING COMPANY

RELIANT ENERGY SERVICES, INC.

RENAISSANCE ENERGY (U.S.), INC.

ROCHESTER GAS \& ELECTRIC CORPORATION

SACRAMENTO MUNICIPAL UTILITY DISTRICT

SAN DIEGO GAS \& ELECTRIC

\section{8 \\ Oct.-Dec.}

376
317
10560
11269

1111

1152

1090

0

659

0

18

0

299

979

2974

0

55

289

0
116

3506

35

2787

190

4175

15675

961

10286

55695

1205

0

11082

3797

0
113

335

10646

910

1195

44
10394

10394
0

671

1541

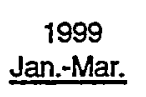

0
3581
1729

9977

285

11141

1078

1078

1011

150

0

256

0

913

293

1941

4621

10

102

361

0

0.

3406

0
2760

278

0

23623

262

4382

15645

51310

851

13

15097

4004

265

0

0
7863

2050

1846

702

10743

215

731

2182

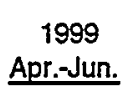

921
3676
0
10978

0
13983

1094

1090

849

0

0
0

0

917

296

1525

3979

0

0

267

0
15

850

0

2776

0

1559

23641

223

11586

13566

50600

0
0

16807

4642

0
0
0
7723
982
15
602
10752
1591
1056
2100
1999
Jul.-Sep.

1380

1380
3724
0

10795

0
17589

1103

1106

858

0

0

0
0

925

299

1639

3361

0

0
166

166
0

0
1193

1193
-0

2841

1756

22695

22994

135

12614

13918

52397

0

17617

4891

0

1676

8089

2061

15

1423

13469

2514

528

2033
1999

Oct.-Dec.

1380

3742

0

8457

610

16576

1053

1108

289

0

0

0

941

299

1248

3861

0

5

234

2702

663

2818

2238

883

24551

55

13928

13941

53204

0

0

16469

4611

0

378

8864

3588

349

4056

10061

3004

1882

(1) Formerly known as Idaho Power Company. Volumes imported during 4th Qtr $98(0.363)$ are too low to appear in the body of Attachments C2 and C3; however, they are included in the total volumes on both Attachments. 


\section{SHORT-TERM IMPORTERS}

Estimated Volumes (MMCF)

SELKIRK COGEN PARTNERS, L.P. SEMPRA ENERGY TRADING CORPORATION SIERRA PACIFIC POWER COMPANY SOUTHERN COMPANY ENERGY MARKETING L.P. SPRAGUE ENERGY CORPORATION

ST. LAWRENCE GAS COMPANY, INC. STAMPEDER ENERGY (U.S.) INC. STATOIL ENERGY SERVICES, INC. SUMAS COGENERATION COMPANY, LP SUNCOR ENERGY INC.

SUNOMA ENERGY CORPORATION TENASKA MARKETING VENTURES TEXACO NATURAL GAS INC. TEXAS-OHIO ENERGY, INC. THE MEAD CORPORATION THE MONTANA POWER COMPANY

THE MONTANA POWER TRADING \& MARKETING COMPANY TRANSCANADA GAS SERVICES INC.

TRANSCO ENERGY MARKETING COMPANY

TXU ENERGY TRADING COMPANY

UNION PACIFIC FUELS, INC.

UNITED STATES GYPSUM COMPANY

UPSTATE ENERGY INC.

US GEN NEW ENGLAND

UTILICORP UNITED INC.

VERMONT GAS SYSTEMS, INC.

WESTERN GAS RESOURCES, INC.

WGR CANADA, INC.

WISCONSIN GAS COMPANY

WISCONSIN PUBLIC SERVICE CORPORATION

\begin{tabular}{|c|c|}
\hline $\begin{array}{c}1998 \\
\text { Oct.-Dec. }\end{array}$ & $\begin{array}{r}1999 \\
\text { Jan-MM }\end{array}$ \\
\hline 16 & \\
\hline 4012 & 583 \\
\hline 2270 & \\
\hline 0 & \\
\hline 0 & \\
\hline 298 & \\
\hline 1258 & \\
\hline 1890 & 1969 \\
\hline 1020 & \\
\hline 5183 & \\
\hline 0 & \\
\hline 1804 & \\
\hline 1535 & \\
\hline 202 & \\
\hline 0 & \\
\hline 622 & \\
\hline 4098 & \\
\hline 69947 & 64681 \\
\hline . 959 & 49 \\
\hline 5271 & \\
\hline 13433 & 1908 \\
\hline 1039 & \\
\hline 0 & \\
\hline 3564 & \\
\hline 181 & 18 \\
\hline 531 & \\
\hline 18 & \\
\hline 0 & \\
\hline 0 & \\
\hline 641 & \\
\hline
\end{tabular}

Totals

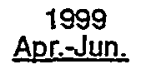

1999
Jul.-Sep.

\section{0}

4422

2713

0

1993

125

799

465

0

4754

0

5150

1204

0

455

0
4000

69001

5070

3796

0

878

289

5463

1836

120

0

5

0

0

0

0

0

0

0

467766
1999

Oct.-Dec.

$\begin{array}{rr}0 & 0 \\ 4460 & 6657 \\ 2504 & 3176 \\ 0 & 276 \\ 493 & 801 \\ 108 & 237 \\ .4483 & 4561\end{array}$

6640

13313

0

5005

0

4864

905

0

455

0

4140

76418

7724

1835

0

929

225

4847

1871

0

0

0

2657

0

0

5109

250

5214

694

0

455

619

5132

70328

10072

2083

0

923

10

2655

3694

245

0

0

270

$\underline{568149}$ 
PURCHASERS OF SHORT-TERM GAS Estimated Volumes Imported (MMCF)

A-G Energy, L.P.

AEC Marketing (USA) Inc.

$A E C$ Storage and Hub

AEP Energy Services

Abitibi Consolidated Sales Corporation

Adams Resources Marketing, Ltd.

Advanced Wood Products

Affiliated Health

Alliance Energy Services

Altrade

Aluminum Company of America

Amerada Hess

Ameren

American Electric Power

American National Power Co.

Amoco Canada Marketing Co.

Amoco Canada Petroleum

Amoco Trading

Anadarko Trading Company

Androscoggin Energy LLC

Aquila Canada Corporation

Aquila Energy Marketing

Aquila Power Corporation

Arco Products Company

Armstrong World Industries, Inc.

Ash Grove Cement

Associated Sand \& Gravel

Avista Corporation

Avista Energy

BC Gas Utility

BHP Coated Steel Corporation

Barret Resources Corporation

Basic American Foods

Bay State Gas Company

Berkshire Gas Company

Big Sky Gas Marketing

Bimingham Steel

Black Hills Energy

Boeing Company

Boise Cascade Corporation

Borden

Boston Gas Company

Bowman Manufacturing

Bullseye Glass

CMS Gas Marketing

CXY Energy Marketing

Cabot Oil

CalPine Fuels Corporation

Cardinal IG Company

Cargill Energy Division
1998

Qct.-Dec.
1999 Jan.-Mar.

23

56

21

7

0

0

14

0

339

0

803

0

0

0

48

4

661

169

0

1209

2867

327

1310

84

98

44

14457

0

0

3500

14

43

751

184

72

433

503

31

3262

0

2

316

462

390

137

0

246

Apr.-Jun.

895

0

0

0

86

8

387

2645

432

0

141

12493

242

1790

3

16

14

5504

16072

16

0

5742

0

184

37

774

144

437.

1119

71

44

13

0

0

1165

567
1999

Jul.-Sep.
15

13599

0

12

36

0

33

12

300

0

1490

(2)$$
\begin{array}{r}
0 \\
13987
\end{array}
$$

13987

0

204

0

0

0

0

448

45

1286

0

0
19

19

9

0

1277

0

0

3981

265

2351

128

204

59

3920

19522

0

90

2593

144

24

17

817

264

455

230

1642

14

50

1

29

278

1067

0

8

0

1006

14255

100

9449

$\circ$

0

0

762

0

112

233

0

0

0

0

0

2620

1131
1999

오.-Dec.

3

13723

0

854

0

172

0

0

5

223

1516

0

156

14

0

4

$0 \ldots$.

1338

0

167

1387

2049

0

2335

0

0

0

6721

14872

3
0
0
0
0
5
0
0
4
0
0

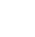

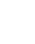

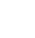

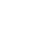

72
0

0

3576

0

132

0

991

0

219

561

0

0

317

0

0

2429

1014

4 
Page - 61

PURCHASERS OF SHORT-TERM GAS

Estimated Volumes Imported (MMCF)

Carthage Energy Services

Cascade Hardwood

Cascade Natural Gas

Central Hudson Gas \& Electric

Central Premix

Chevron Natural Gas Services, Inc.

Chevron U.S.A. Inc.

Chiquita Processed Foods

Cibola Corporation

Cintas

City of Ellensburg, WA

City of Long Beach, CA

City of Perham, MN

City of Two Harbors, MI

Cleco

Cleveland Cliffs

Clinton Gas Marketing .

CoEnergy Trading Company

Coast Energy Group

Coastal Gas Marketing Company

Cogen Energy Technology

Colonial Energy, Inc.

Colonial Gas Company

Columbia Beverage

Columbia Energy Services

Columbia Natural Gas

Columbia Steel

ComGas

Commonwealth Edison

Commonwealth Energy Services

Commonwealth Gas Company

ConAgra Energy

Connecticut Natural Gas

Conoco Inc.

Consolidated Edison

Consumers Energy Company

Cook Inlet

Coral Energy Resources

Coral Redwood

Com Plus

Comerstone Gas Resources, Inc.

Corporation

Crockett Cogeneration

DFAS-Columbus Center

Darling Delaware

Darling International

Defense Department

Delmarva Power \& Light Company

Destec Gas Services

Detroit Edison Company
1998

Oat.-Dec.

1999 Jan.-Mar.

1999
Apr.-Jun.

1999

Jul.-Sep.

1999

284

15500

352

51

31

11

50

65

0

9216

510

8
26

5

251

13

0

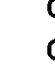

27

26

0
2738

4843

842

1172

1092

30

115

0

2931

0

13

6
0

- 0

249

1956
733
.12
3789
64
7
29
8
0
24675

7

248

0

74

0

0

0

71

1795

5674

1126

38

30

4

298

15

0

0

493

0

37

0

81
0

2848

2387

222

935

907

29

0
8

1178

1178
0

6

5

56

29

0
303
3

697

82

0

122

14

23633

307

10

0

23

0

128

175

2074

9853

787

0

20

16

688

0

32

0

411

0
113

113
0

15

1
3152

3125

737

1718

252

0

0

2012

423

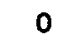

26

23

1948

0

386
697

$\begin{array}{rr}0 & 269 \\ 0 & 0 \\ 3689 & 8104 \\ 75 & 222 \\ 0 & 0 \\ 0 & 0 \\ 0 & 0 \\ 0 & 0\end{array}$

19455

25678

338

0

1470

$$
0
$$

18

31

408

417

1301

11278

0

0

0

1731

0

0

276

441

50

276

0

$\begin{array}{rr}235 & 182 \\ 24 & 1\end{array}$

$2836 \quad 3104$

$4799 \quad 1770$

$778 \quad 456$

$5093 \quad 3618$

191

157

0

0

3224

1999

1987

1814

380 
PURCHASERS OF SHORT-TERM GAS Estimated Volumes Imported (MMCF)

Draper Farms

Duke Energy Marketing Canada

Duke Energy Resource Management Co.

Duke Energy Trading \& Marketing

Duke Field Services

Dynegy Inc.

Dynegy Marketing \& Trade

Dyno Overlays, Inc.

Dyno Polymers

ERI Services, Inc.

ESCO Corporation

Eagle Picher

Eagle Veneer

El Paso Energy Marketing

Elanbaas

Encina Gas Marketing

Energy Express

Energy Masters Intemational, Inc.

Energy North Inc.

Energy USA

Energy West Inc.

Engage Energy U.S. LP

Enline Energy Solutions

Enron Capital \& Trade Resources

Enron Gas Marketing

Enron Gas Services

Enron Intemational Gas Sales Co.

Enron North America Corp.

Ensearch Gas Company

Enserch Energy Services, inc.

Enserco Energy, Inc.

Equitable Resources Marketing

Essex County Gas Company

Eugene Water \& Electric Board

Evanite Fiber

Fall River Gas Company

Famland Industries

Faultine

Fircrest Schools

Fitchburg Gas \& Electric Company

Florida Power \& Light Company

Fort James Corporation

Fuel Imbalance

Fuel Use

Fullmer Forest Products

GAS Senvices

Gaslantic

General Chemical

Georgia Pacific Corporation

Great Westem Malting

\begin{tabular}{|c|c|c|c|c|}
\hline $\begin{array}{c}1998 \\
\text { Oct.-Dec. }\end{array}$ & $\begin{array}{c}1999 \\
\text { Jan.-Mar. }\end{array}$ & $\begin{array}{c}1999 \\
\text { Apr.-Jun. }\end{array}$ & $\begin{array}{c}1999 \\
\text { Jul.-Sep. }\end{array}$ & $\begin{array}{c}1999 \\
\text { Oct.-Dec. }\end{array}$ \\
\hline
\end{tabular}

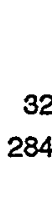

$\begin{array}{rr}8 & 8 \\ 186 & 0 \\ 3047 & 3638 \\ 21755 & 29616 \\ 0 & 569 \\ 4100 & 3460\end{array}$

1848

3706

7957

5346

40

4574

16

30

13

1889

0

0

25

60

63

0

63

5170

0
26497

1524

17

0

0

151

1779

261

0

0

0
121

34

1059

2

39

0
951

1073

1012

0

1120

2

0

0

39
Jul.Sep.

$\begin{array}{rr}0 & 0 \\ 0 & 0 \\ 0 & 0 \\ 53870 & 48340 \\ 2203 & 1797 \\ 5910 & 1982 \\ 5085 & 3164 \\ 0 & 0 \\ 0 & 0 \\ 0 & 0 \\ 0 & 0 \\ 0 & 0 \\ 0 & 0\end{array}$

2687

2876

643

0

0

0

134

58

685

200

33438

14869

37

33340

37943

105

1527

35685

455

524

0

0

0

1036

606

16

2

57

148

164

37

905

0

876

17

48

23

1474

1134

938

0

0

138

2713

1923

32

0

120

404

353

0

1

0

0

0

0

193

847

0

38

45

0

922

0

15

0

31

30

10

89






\section{PURCHASERS OF SHORT-TERM GAS Estimated Volumes Imported (MMCF)}

\begin{tabular}{|c|c|c|c|c|c|}
\hline$\cdot$ & $\begin{array}{c}1998 \\
\text { Oct.-Dec. }\end{array}$ & $\begin{array}{c}1999 \\
\text { Jan.-Mar. }\end{array}$ & $\begin{array}{c}1999 \\
\text { Apr.-Jun. }\end{array}$ & $\begin{array}{c}1999 \\
\text { Jul.-Sep. }\end{array}$ & $\begin{array}{c}1999 \\
\text { Oct.-Dec. }\end{array}$ \\
\hline GulfMark Energy Inc. & 1025 & 739 & 0 & 0 & 0 \\
\hline H.Q. Energy Services (U.S.) Inc. & 0 & 0 & 921 & 1380 & 1380 \\
\hline HL Power & 47 & 53 & 64 & 125 & 120 \\
\hline Haggens Market & 0 & 0 & 1 & 0 & 0 \\
\hline Hesse Gas Company & 359 & 3509 & 0 & 3649 & 3667 \\
\hline Hexcel & 4 & 1 & 10 & 0 & 0 \\
\hline Holnam Inc. & 490 & 246 & 76 & 33 & 0 \\
\hline Holy Rosary Medical Center & 0 & 0 & 4 & 0 & 0 \\
\hline Holyoke Gas Company & 13 & 17 & 0 & 0 & 0 \\
\hline Honeymead Products Company & 0 & 59 & 61 & 92 & 92 \\
\hline Howard Energy Company & 5259 & 5043 & 611 & 1672 & 455 \\
\hline IBP & 0 & 0 & 11 & 34 & 34 \\
\hline IDA Corporation Energy Solutions L.P. & 0 & 0 & 23 & 0 & 9 \\
\hline IGI Resources & 1545 & 2008 & 3050 & 3351 & 2673 \\
\hline idacorp Energy Solutions L.P. & 0 & 0 & 444 & 448 & 151 \\
\hline Idaho Power Company & 86 & 4 & 78 & 1 & 0 \\
\hline Indeck Energy Services of Oswego & 1128 & 1078 & 1094 & 1103 & 1053 \\
\hline Indeck Energy Services of Yerkes & 1152 & 1078 & 1090 & 1106 & 1108 \\
\hline Intalco Aluminum Corporation & 161 & 227 & 203 & $23 \dot{5}$ & 264 \\
\hline Interenergy Corporation & 1090 & 1011 & 849 & 858 & 289 \\
\hline Intermountain Gas Company & 0 & 0 & 0 & 3549 & 4000 \\
\hline Interox & 66 & 0 & 0 & 0 & 0 \\
\hline Interstate Power Company & 0 & 0 & 25 & 0 & 0 \\
\hline Iroquois Energy Management & 6 & 0 & 0 & 0 & 0 \\
\hline J.H. Gypsum & 275 & 101 & 313 & 0 & 0 \\
\hline J.R. Simplot Company & 4218 & 8158 & 4923 & 0 & 0 \\
\hline Jeld Wen & 0 & 9 & 0 & 0 & 0 \\
\hline Johnson Control & 11 & 21 & 11 & 0 & 0 \\
\hline Jorgenson Forge & 45 & 69 & 86 & 0 & 0 \\
\hline K-N Gas Marketing & 0 & 6 & 294 & 32 & 417 \\
\hline KN Energy & .493 & 0 & 0 & 22 & 125 \\
\hline KN Marketing L.P. & 0 & 493 & 831 & 325 & 766 \\
\hline Kaztec Energy & 258 & 250 & 84 & 42 & 0 \\
\hline Kaztex Energy Management & 850 & 1374 & 847 & 453 & 720 \\
\hline KeySpan Gas East Corporation & 0 & 320 & 500 & 605 & 375 \\
\hline Kimball Energy Corporation & 2 & 546 & 0 & 27 & 208 \\
\hline Kimball Trading Company, L.L.C. & 1141 & 15 & 11 & 1 & 0 \\
\hline Kind \& Knox Gelatine, Inc. & 46 & 67 & 0 & 0 & 0 \\
\hline Kings Meat & 17 & $\cdot 16$ & 16 & 0 & 0 \\
\hline Koch Energy Trading & 3 & 273 & 25 & 270 & 369 \\
\hline Koch Gas Services Company & 434 & 6 & 143 & 154 & 0 \\
\hline L'Energia L.P. & 19 & 128 & 0 & 0 & 0 \\
\hline LG\&E Natural Marketing Inc. & 581 & 0 & 0 & 0 & 0 \\
\hline LG\&E Westmoreland & 163 & 0 & 0 & 0 & 0 \\
\hline Lakeside & 75 & 19 & 127 & 0 & .0 \\
\hline Lamb Weston & 0 & 0 & 479 & 0 & 0 \\
\hline Lane Community College & 0 & 0 & 4 & 0 & 0 \\
\hline Legacy Health System & 26 & 10 & 36 & 0 & 0 \\
\hline Lehigh Portland Cement Company & 29 & 58 & 0 & 0 & 0 \\
\hline Lignotech & 0 & 0 & 39 & 0 & 0 \\
\hline
\end{tabular}




\section{PURCHASERS OF SHORT-TERM GAS}

Estimated Volumes Imported (MMCF)

Long Island Lighting Company

Longview Fibre

Louisiana Pacific

M.A. Segale

MA Municipal Wholesale Electric Co.

MCV Gas

MGI

Madison Gas \& Electric

Marketspan Gas Corporation

Masonite Corporation

Massachusetts Municipal Wholesale Electric Co.

Masspower

McCain Foods, Inc.

Mead Paper

MichCon Trading

Michigan Consolidated Gas Company

Mid American Natural Resources

MidAmerican Energy Company

Midcon Gas Services Corporation

Middleborough Gas Company

Midland Cogeneration Venture

Mieco

Milford Power, L.P.

Minnegasco

Minnesota Com Processors

Minnesota Gas Company

Montana Power

Montana Resources

Montana-Dakota Utilities Co.

Murphy Gas Gathering

N.Y. State Electric \& Gas

NESI Energy Marketing

NORPAC

NUI Corporation

National Fuel Gas Distribution

National Fuel Marketing Company, L.L.C.

National Fuel Resources

National Gas \& Electric

National Gas Resources

National Steel Corporation

Natural Gas Transportation Services

Naumes

Neste Resins

Neste Trading USA Inc.

Nestle USA, Inc.

Nevàda Power Company

New England Power Company

New Jersey Natural Gas

Niagara Mohawk

Nicor Gas Company

\begin{tabular}{|c|c|}
\hline $\begin{array}{c}1998 \\
\text { Oct.-Dec. } \\
\end{array}$ & $\begin{array}{c}1999 \\
\text { Jan.-Mar }\end{array}$ \\
\hline 1086 & 8 \\
\hline 0 & 0 \\
\hline 0 & 0 \\
\hline 31 & 0 \\
\hline 0 & 3 \\
\hline 150 & 0 \\
\hline 0 & 3423 \\
\hline 0 & 0 \\
\hline 12 & 0 \\
\hline 0 & 0 \\
\hline 0 & 0 \\
\hline 37 & 0 \\
\hline 0 & 0 \\
\hline 0 & 217 \\
\hline 248 & 0 \\
\hline 5661 & 6156 \\
\hline 0 & 0 \\
\hline 1349 & 0 \\
\hline 153 & 0 \\
\hline 8 & 7 \\
\hline 495 & 0 \\
\hline 0 & 14 \\
\hline 564 & 655 \\
\hline 3642 & 5907 \\
\hline 879 & 986 \\
\hline 155 & 450 \\
\hline 1090 & 305 \\
\hline 101 & 50 \\
\hline 273 & 1184 \\
\hline 299 & 293 \\
\hline 196 & 23 \\
\hline 353 & 280 \\
\hline 1 & 1 \\
\hline 7 & 0 \\
\hline 602 & 1711 \\
\hline 0 & 0 \\
\hline 323 & 435 \\
\hline 531 & 461 \\
\hline 0 & 0 \\
\hline 0 & 10 \\
\hline 0 & 0 \\
\hline 0 & 0 \\
\hline 0 & 0 \\
\hline 7 & 7 \\
\hline 0 & 0 \\
\hline 174 & 65 \\
\hline 3564 & 4916 \\
\hline 82 & 0 \\
\hline 0 & 0 \\
\hline 321 & 724 \\
\hline
\end{tabular}

1999
Jul.-Sep.

1986

215

0

36

0

0
9631

0

0

0
62

116

102

910

0

5403

318

0

3

258

76

554

1347

623

463

1

14

917

296

795

0

31

2822

0

669

460

0

0

0

58

5

0

228

33

5472

0
78

3095

306

0

0

2172

0

2310

464

0

0

82

0

0

0

0

9

4847

192

69

4197
Jul.-Sep, Oct.Dec.

$32 \quad 800$

$0 \quad 0$

$71 \quad 6$

$0 \quad 0$

$\begin{array}{ll}0 & 0\end{array}$

0

$12472 \quad 13928$

02

$0 \quad 0$

20

$0 \quad 0$

$0 \quad 0$

$0 \quad 0$

$915 \quad 1092$

$0 \quad 0$

$6861 \quad 5789$

$\begin{array}{rr}0 & 177 \\ 417 & 255\end{array}$

$0 \quad 0$

$0 \quad 0$

$47 \quad 0$

$65 \quad 46$

$182 \quad 559$

1362459

$310 \quad 529$

$460 \quad 1409$
754

0

1130

299

331

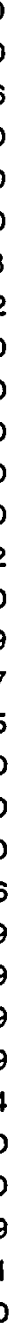


PURCHASERS OF SHORT-TERM GAS Estimated Volumes Imported (MMCF)

Nipsco Energy Trading Corporation Noble Gas Marketing

Noram Energy Services

North American Energy Inc.

North Attleboro Gas Company

Northeast Energy Associates

Northeast Gas Markets

Northeast Utilities

Northem Border Pipeline

Northem Indiana Public Service

Northem States Power (MN)

Northem States Power (WI)

Northem States Power Company

Northem Utilities, Inc.

Northshore Gas Company

Northwest Alloys

Northwest Cooperage

Northwest Natural Gas Company

OCE Energy Resources

OGE Energy Resources

Occidental

Ocean Kayak, Inc.

Ocean State Power

Omaha Utilities District

Onyx Gas Marketing Company

Orange \& Rockland Utilities

Ore-Ida

Oregon Potato

Oregon State University

Oregon Steel Mills

Oroweat

Overall Laundry

Oxy, Inc.

PABST

PG\&E Core Procurement Dept.

PG\&E Energy Trading

PG\&E Gas Transmission NW

PNGTS - Linepack

$P Q$ Corporation

Pace Energy, L.L.C.

Pacific Gas \& Electric (UEG)

Pacific Gas \& Electric Company

Pan-Alberta Gas (U.S.) Inc.

Pancanadian Energy Services

Pancanadian Petroleum Ltd.

Panhandle Gas Services

Peco

Pendelton Woolen

Pennford Products Company

Peoples Gas, Light, \& Coke

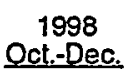

\author{
1999 \\ Jan.-Mar.
}

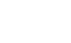

$$
77
$$$$
775
$$$$
289
$$

1

0

0

0

54

0

251

0

36

3335

84

11

4589

529

0

219

. 0

0

309

190

78

0

0

89

297

9

15

0

34

55927

5103
145

145
0

0

5

858

5728

0
1633

0

532

18

0

3624

784
361

13

0

3

0

24

35

3146

243

.11

4248

604

1338

374

0

55

391

278

145

0

0

72

214

0

15

0
24

51569

4421

0

265

0

0
57

57
3463

2

1593

1351

0

0

0

2584

1999
Apr.-Jun.

1999
Jul.-Sep.

1999

Oct.-Dec.

0

0
259

25

0

272

390

267

842

0

234

166

0
75

0

300
0
0
0
15
39
0
5380
245
11
1592
0
1082

151

2

0

0

114

193

110

45

86

25

17

79

50715

3448

0

0

42

0
36

258

0

2544

0

0

0
35

19

4598
0

1886

\section{0}

0

0

663

1645

5364

253

0

7127

0

1301

72

0

0

0

1756

0

0

0

0

$\begin{array}{rr}146 & 146 \\ 0 & 0\end{array}$

$0 \quad 0$

$\begin{array}{rr}0 & 317 \\ 0 & 0\end{array}$

$52436 \quad 53204$

$6225 \quad 2243$

50

0

0

0

0

25

4337

. 0

0

0

0

0

5596 
PURCHASERS OF SHORT-TERM GAS Estimated Volumes Imported (MMCF)

Peoples Natural Gas Company

Perry Gas Company

Phibro Oil \& Gas Inc.

Phoenix Chemical

Pictsweet

Pierce Transit

Pittsfield Generating Plant

Poco Marketing

Poco Petroleum

Ponderosa

Portland Community College .

Portland General Electric Company

Portland State University

Potsdam College

ProEnergy

Proctor and Gamble Company

Progas

Providence Energy Services Company

Public Service Gas \& Electric

Public Service of New Mexico

Puget Sound Energy

Questar Energy Trading Co.

Quick Trade

RDO Foods Company

Rainy River

Redhook

Reliant Energy Services, Inc.

Renaissance Energy (U.S.) Inc.

Rhone-Poulenc

Rochester Gas \& Electric Company

Roseburg Forest

Roseburg Lumber

Rozsa Petroleum Ltd.

SCANA Energy Marketing

SEMCO Energy Services

Sacramento Municipal Utility Dist.

San Diego Gas \& Electric Company

Santiam Forest Products

Seattle Snohomish

Selkirk Cogeneration Partners

Sempra Energy Tradirig Corp.

Sierra Pacific industries

Sierra Pacific Power Company

Simpson Tacoma Kraft Company

Sioux City Brick \& Tile

Sith Mystic, LLC

Sithe Energy

Sithe New Boston, LLC

Smurfit Newsprint Corporation

Sonat Marketing Company
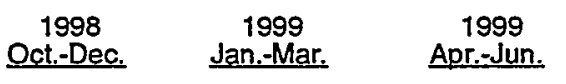

1999
Jul.-Sep.

1999
Oct.-Dec.

444

67

487

11

21

5

3467

0

4408$$
\begin{array}{r}
4 \\
51
\end{array}
$$$$
10
$$$$
14
$$$$
0
$$$$
11502
$$$$
51
$$$$
733
$$$$
38
$$$$
53
$$$$
14
$$$$
10394
$$$$
2
$$$$
0
$$$$
37
$$$$
\begin{array}{r}
5 \\
322
\end{array}
$$$$
625
$$$$
1072
$$$$
1660
$$$$
19
$$$$
18
$$$$
16
$$$$
1049
$$$$
151
$$$$
4530
$$$$
179
$$$$
20
$$$$
\begin{array}{r}
0 \\
154
\end{array}
$$$$
\begin{array}{r}
154 \\
0
\end{array}
$$

455

$$
0
$$

Apr-Jun.

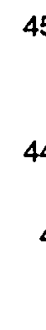

457

3

0

445

0

49

0
0

3470

3449

0

0
7096

5

49

3

11

0

0

12034

462

259

3

1660

10743

1
215

0

0

56

574

845

2201

20

1

240

2058

0

4785

138

29

0

0

79

60

2147
472

471

2

0

369

0

3569

0

0

0

7490

0

49

0

201

0

0

61

0

11348

5

156

0

0

0

10370

10061

12

3004

0

0

0

65

0

171

1930

2062

0

20

6098

0

3694

0

0

0

0

$$
0
$$

62 
PURCHASERS OF SHORT-TERM GAS Estimated Volumes Imported (MMCF)

South Jersey Gas Company

South Jersey Resources Inc.

Southem Califomia Gas Company

Southem Company Energy

Southem Connecticut Gas Company

Southem Indiana

Southwest Gas Corporation

Sprague Eneirgy

St. Joseph's Hospital

St. Lawrence University

Star Natural Gas Company

State University of New York

Statoil Energy Services, inc.

Sugiyo USA, Inc.

Sumas Cogeneration

TM Star

TPC Coppration

TXU Energy Trading Company

Technal Corporation

Tejas Power

Tenaska Gas Company

Tenaska Marketing Ventures

Tenaska Washington Partners

Terra International

Tesoro Northwest Company

Tessenderlo Kèly, Inc.

TexEnergy

Texaco Gas Marketing Inc.

Texaco Natural Gas

Texas-Ohio Gas, Inc.

Texex Energy Partners

The Montana Power Company

Todds Shipyards

Torch Gas L.C.

Tosco Refining Company

Tractebel

TransCanada Energy Marketing USA

TransCanada Gas Services

TransCanada Pipelines Limited

TransCanada Power (Castleton) LLC

TransOcean Products, Inc.

Tristar Gas Marketing

Tucci \& Sons

U.S. Energy Services

U.S. Gas Transportation Inc.

U.S. Generating Company

U.S. Generating New England

US Generating

US Oil \& Refining

Union Gas

\section{Oct.-Dec.}

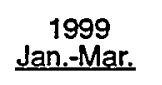

1999
Apr.Jun.

1999
Jul.-Sep.

1999 1

$\begin{array}{rr}1 & 0 \\ 0 & . \\ 2443 & \\ 1755 & 24 \\ 98 & 1816 \\ 0 & 123 \\ 25 & 0 \\ 15 & 12 \\ 0 & 6 \\ 23 & 0 \\ 0 & 35 \\ 8 & 0 \\ & \end{array}$

0

1020

37

235

0

2
307

8

3142

327

684

0
0

288

2060

178

0

0
18

0

913

3517

2214

471

0

321

1
1881

1881
110

0

0

69

48

27

\section{Apro}

1939

0

0

697

0

0
0

.3305

150

0

0

16

0

429

3458

3112

0

0

0

397

0

2292

0

0

0

55

33

0
502

59

3072

0

796

6

14

35

78

1156

3

0

0

413

3

0

2491

\section{0}

12867

4385

704

3

0
0

2169

148

0

17

18

556

218

305

2531

2698

0

0

0

1285

0

26

42

0

51

0
312

0

31

379

0

35

302

0

592

0

0

0

0

1841

0

27

3758

103

0
660

Oct.-Dec.

$$
\begin{array}{r}
0 \\
0 \\
3 \\
6538 \\
386 \\
42 \\
745 \\
0 \\
0 \\
12 \\
93 \\
0 \\
2715 \\
0 \\
0 \\
0 \\
0 \\
2948 \\
0 \\
205 \\
0 \\
5734 \\
321 \\
0 \\
892 \\
0 \\
16 \\
0 \\
1148
\end{array}
$$$$
1124 \cdot 1148
$$$$
0 \quad 0
$$$$
\begin{array}{ll}
0 & 49
\end{array}
$$$$
\begin{array}{ll}
0 & 0 \\
0 & 0
\end{array}
$$$$
319 \quad 562
$$$$
108 \quad 283
$$$$
4550
$$$$
1639 \quad 1513
$$$$
2759 \quad 3239
$$$$
0 \quad 0
$$$$
383 \quad 73
$$$$
0 \quad 0
$$$$
0 \text { o }
$$$$
0 \quad 0
$$$$
803 \quad 1057
$$$$
636 \quad 161
$$$$
16 \quad 32
$$$$
0
$$$$
0
$$$$
0
$$ 
PURCHASERS OF SHORT-TERM GAS Estimated Volumes Imported (MMCF)

Union Pacific Fuels

Union Pacific Resources

Unisea Foods

United Foods

United States Gypsum

University of Oregon

Unocal

Upstate Energy, Inc.

Utilicorp Retail Services

Utilicorp United

Vailey Gas Company

Valley Medical

Various CA State Markets

Various CO State Markets

Various IA State Markets

Various IL State Markets

Various MA State Markets

Various MI State Markets

Various MN State Markets

Various MT State Markets

Various NE State Markets

Various NJ State Markets

Various NV State Markets

Various NY State Markets

Various OR State Markets

Various TX State Markets

Various WA State Markets

Various WI State Markets

Vermont Gas Systems, Inc.

Virginia Power

WP Natural Gas

WPS Energy Services, Inc.

Washington Natural Gas Company

Washington Water Power Company

West Linn Paper Company

Westcoast Gas Services (U.S.A.) Inc.

Westem Gas Marketing Inc.

Western Gas Resources

Westem Gas Services

Weyerhauser

Wild Goose Storage

Wildhorse Energy Partners, L.L.C.

Willamette

Williams Energy Company

Wisconsin Electric

Wisconsin Fuel \& Light

Wisconsin Gas

Wisconsin Power \& Light

Wisconsin Public Service Corp.

Woodworth

\begin{tabular}{|c|}
\hline $\begin{array}{c}1998 \\
\text { Oct.-Dec. }\end{array}$ \\
\hline
\end{tabular}

13453

19093

326

7

0

$12 \quad 20$

$1093 \quad 836$

$87 \quad 67$

461

197

81

2508

1351

28

28

15533

3509

3911

6885

0

1579

- 204

690

987

0

912

13501

529

0
1592

2859

531

49

0

822

0

4244

358

209

0

1780

6

0
105

105
74

1086

566

614

0

1836

1064

1704

$$
\begin{array}{r}
33 \\
52
\end{array}
$$

14205

3037

4320

22735

0

2159

273

628

1708

0

898

19855

6

10
810

1104

120

11

580

61

0

309

0

0

871

0

0

0

0
686

1476

0

0

101

1021

725

41

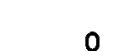

0

3

18

878

59

940

835

0

426

6
56

14360

2872

4825

24293

29

2638

398

275

933

491

910

15334

53

0
1714

432

0

75

0

767

0

0

93

0

0

97

0

0

13

0

809

9

34

0

99

1281

688

15

$\begin{array}{rr}0 & 0 \\ 0 & 0 \\ 0 & 0 \\ 0 & 0 \\ 929 & 923 \\ 0 & 0 \\ 744 & 336 \\ 1117 & 10 \\ 0 & 0 \\ 3287 & 5446 \\ 0 & 0 \\ 0 & 0 \\ 13471 & 13185 \\ 2992 & 0 \\ 4785 & 4373 \\ 18813 & 16123 \\ 5906 & 5902 \\ 2606 & 659 \\ 122 & 2068 \\ 48 & 3969 \\ 316 & 0 \\ 149 & 58 \\ 920 & 507 \\ 15365 & 23820 \\ 66 & 964 \\ 0 & 0 \\ 1488 & 4739 \\ 118 & 420 \\ 0 & 245 \\ 30 & 6 \\ 709 & 010 \\ 1185 & 0\end{array}$

1185

0

0

59

5

0

911

0

733

43

0

760

406

116

0

4432

1307
735

0
0

0

0

0

336

10

5446

0

185

4373

6123

5902

068

3969

58

507

3820

0

739

420

45

1012

0

0

0

0

9

92

639

16

0

338

309

.49

18

1600

1078

252 


\section{PURCHASERS OF SHORT-TERM GAS}

Estimated Volumes imported (MMCF)

$X$ Energy, Inc.

Yankeo Gas Services

Totals

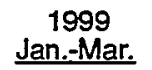

1998
Oct-Dec

1

853

442108
1999

Apr.-Jun.

0

861

467766

\begin{tabular}{cc}
$\begin{array}{c}1999 \\
\text { Jul.-Sep. }\end{array} \quad$ Oct.-Dec. \\
\hline
\end{tabular}

0

870

0

577

$\underline{568149} \quad \underline{556192}$ 


\section{SHORT-TERM SALES \\ YEAR: 1999 Quarter: Four \\ Estimated Volumes (MMCF) \& Prices (\$/MMBTU)}

Importer
Seller
Purchaser/ End User
AEC MARKETING (USA) INC.
AEC Marketing (USA) InC.
AEC Marketing (USA)

AEC WEST LTD.

AEC West Ltd.

$A E C$ Marketing (USA) Inc.

Port of Morgan, MT

ALCOA, INC.

Amoco Canada Petroleum Company Ltd.

Aluminum Company of America

Port of Morgan, MT

ALCOA, INC.

Renaissance Energy Ltd.

Aluminum Company of America

Massena, New York

AMOCO CANADA MARKETING CORPORATION

Amoco Canada Petroleum Company Ltd.

American Electric Power

$$
\text { Eastport, Idaho }
$$

AMOCO CANADA MARKETING CORPORATION

Amoco Canada Petroleum Company Ltd.

Amoco Canada Marketing Co.

Sumas, Washington

AMOCO CANADA MARKETING CORPORATION

Amoco Canada Petroleum Company Ltd.

Amoco Trading

Eastport, Idaho

AMOCO CANADA MARKETING CORPORATION

Amoco Canada Petroleum Company Ltd.

Amoco Trading

Niagara Falls, NY

AMOCO CANADA MARKETING CORPORATION

Amoco Canada Petroleum Company Ltd.

Amoco Trading

Waddington, NY

AMOCO CANADA MARKETING CORPORATION

Amoco Canada Petroleum Company Ltd.

Cascade Natural Gas

Sumas, Washington

AMOCO CANADA MARKETING CORPORATION

Amoco Canada Petroleum Company Ltd.

Coast Energy Group

Eastport, Idaho

AMOCO CANADA MARKETING CORPORATION

Amoco Canada Petroleum Company Ltd.

Consolidated Edison
$0 \quad$ N/A

127

2.99

- N/A

127

2.99

October
Vol. Povember
Price

December

Vol. Price

Quarterly

Total

Avg. Price

$\begin{array}{llllllll}2146 & 2.38 & 2053 & 2.88 & 2146 & 2.14 & 6346 & 2.46\end{array}$

$\begin{array}{llllllll}2484 & 2.12 & 2329 & 2.66 & 2565 & 1.78 & 7378 & 2.17\end{array}$

$\begin{array}{llllllll}288 & 2.40 & 273 & 3.01 & 289 & 2.49 & 850 & 2.63\end{array}$

$\begin{array}{llllllll}0 & \text { N/A } & 3 & 2.99 & 10 & 2.61 & 14 & 2.71\end{array}$

$\begin{array}{llllllll}0 & \text { N/A } & 1 & 2.99 & 0 & \text { N/A } & 1 & 2.99\end{array}$

$\begin{array}{llllllll}4 & 2.76 & 0 & \text { N/A } & 57 & 2.61 & 61 & 2.62\end{array}$

$\begin{array}{llllllll}0 & \text { N/A } & 0 & \text { N/A } & 11 & 2.61 & 11 & 2.61\end{array}$

$\begin{array}{llllllll}0 & \text { N/A } & 146 & 2.99 & 0 & \text { N/A } & 146 & 2.99\end{array}$

$\begin{array}{llllllll}0 & \text { N/A } & 14 & 2.99 & 0 & \text { N/A } & 14 & 2.99\end{array}$

$\begin{array}{llllllll}954 & 2.76 & 923 & 2.99 & 954 & 2.61 & 2831 & 2.78\end{array}$ $\begin{array}{llllllll}191 & 3.28 & 221 & 3.73 & 254 & 2.85 & 666 & 3.27\end{array}$
Niagara Falls, NY 


\section{SHORT-TERM SALES}

YEAR: 1999 Quarter: Four

Estimated Volumes (MMCF) \& Prices (\$ / MMBTU)

Importer

Seller

Purchaser/End User
Point

Entry

AMOCO CANADA MARKETING CORPORATION

Amoco Canada Petroleum Company Ltd.

Cook Inlet

October

Vol. Price
November

Vol. Price
December

Vol. Price
Quarterly

Total

Vol. ${ }^{\text {Avg. }}$ Price

AMOCO CANADA MARKETING CORPORATION

Amoco Canada Petroleum Company Ltd.

Cook Inlet

Sumas, Washington

$11 \quad 2.76$

- N/A

0 NIA

$11 \quad 2.76$

AMOCO CANADA MARKETING CORPORATION

Amoco Canada Petroleum Company Ltd.

Coral Energy Resources

$10 \quad 2.76$

o N/A

- N/A

10

2.76

AMOCO CANADA MARKETING CORPORATION

Amoco Canada Petroleum Company Ltd.

Crockett Cogeneration

Eastport, Idaho

$1184 \quad 2.76$

$956 \quad 2.99$

$1084 \quad 2.61$

3224

AMOCO CANADA MARKETING CORPORATION

Amoco Canada Petroleum Company Ltd.

Enron Capital \& Trade Resources

Noyes, Minnesota

$10 \quad 2.76$

o N/A

$0 \quad$ N/A

10

2.76

AMOCO CANADA MARKETING CORPORATION

Amoco Canada Petroleum Company Ltd.

IGI Resources

Eastport, Idaho

0

N/A

$205 \quad 2.99$

$248 \quad 2.61$

453

2.78

AMOCO CANADA MARKETING CORPORATION

Amoco Canada Petroleum Company Ltd.

KN Marketing L.P.

Noyes, Minnesota

$5 \quad 2.76$

- N/A

$0 \quad$ N/A

$5 \quad 2.76$

AMOCO CANADA MARKETING CORPORATION

Amoco Canada Petroleum Company Ltd.

Montana-Dakota Utilities Co.

Port of Morgan, MT

$0 \quad$ N/A

$97 \quad 2.99$

$93 \quad 2.61$

190

2.80

AMOCO CANADA MARKETING CORPORATION

Amoco Canada Petroleum Company Ltd.

Northwest Natural Gas Company

Sumas, Washington

251

2.76

243

2.99

$251 \quad 2.61$

745

2.78

AMOCO CANADA MARKETING CORPORATION

Amoco Canada Petroleum Company Ltd.

Pacific Gas \& Electric Company

Eastport, Idaho

O N/A

$3 \quad 2.99$

$0 \quad N / A$

$3 \quad 2.99$

AMOCO CANADA MARKETING CORPORATION

Amoco Canada Petroleum Company Lid.

Puget Sound Energy

Sumas, Washington

754

2.76

729

2.99

$754 \quad 2.61$

2237

2.78

AMOCO CANADA MARKETING CORPORATION

Amoco Canada Petroleum Company Litd.

Sacramento Municipal Utility Dist.

Eastport, Idaho

110

2.76

$14 \quad 2.99$

o N/A

124

2.79 


\section{SHORT-TERM SALES \\ YEAR: 1999 Quarter: Four \\ Estimated Volumes (MMCF) \& Prices (\$/MMBTU)}

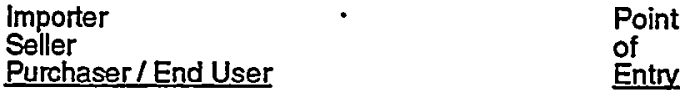

AMOCO CANADA MARKETING CORPORATION

Amoco Canada Petroleum Company Lto.

Sempra Energy Trading Corp.

Eastport, Idaho

$0 \quad$ N/A

$105 \quad 2.99$

$0 \quad$ N/A

Eastport, Idaho

Amoco Canada Petroleum Company Ltd.

Sierra Pacific Power Company

AMOCO CANADA MARKETING CORPORATION

Amoco Canada Petroleum Company Ltd.

Southem Company Energy

Eastport, Idaho

$556 \quad 2.76$

$9 \quad 2.76$

$0 \quad$ N/A

$15 \quad 2.76$

$2 \quad 2.99$

Sumas, Washington

Pittsburg, NH

$0 \quad$ N/A

$4 \quad 3.21$

$0 \quad$ N/A

4

3.21

ANDROSCOGGIN ENERGY LLC

Coral Energy Canada Inc.

Androscoggin Energy LLC

St. Clair, Michigan

- N/A

$4 \quad 2.86$

$0 \quad$ N/A

4

2.86

ANDROSCOGGIN ENERGY LLC

Renaissance Energy Ltd.

Androscoggin Energy LLC

Pittsburg, NH

- N/A

$15 \quad 3.01$

$17 \quad 2.92$

32

2.96

ANDROSCOGGIN ENERGY LLC

Renaissance Energy Ltd.

Androscoggin Energy LLC

St. Clair, Michigan

- N/A

$4 \quad 2.35$

$11 \quad 2.50$

14

2.46

ANDROSCOGGIN ENERGY LLC

Sempra Energy Trading

Androscoggin Energy LLC

Pittsburg, NH

- N/A

o N/A

102

2.70

102

2.70

ANDROSCOGGIN ENERGY LLC

Sempra Energy Trading

Androscoggin Energy LLC

St. Clair, Michigan

AQUILA ENERGY MARKETING CORPORATION

Aquila Canada Corporation

Amoco Trading

Eastport, Idaho $\begin{array}{llllllll}0 & \text { N/A } & 12 & 2.51 & 0 & \text { N/A } & 12 & 2.51\end{array}$

$\begin{array}{llllllll}0 & \text { N/A } & 55 & 3.02 & 0 & \text { N/A } & 55 & 3.02\end{array}$ 
SHORT-TERM SALES

YEAR: 1999 Quarter: Four

Estimated Volumes (MMCF) \& Prices (\$ / MMBTU)

importer

Seller

Pumchaser/EndUser

Point

Entry

AQUILA ENERGY MARKETING CORPORATION

Aquila Canada Corporation

Avista Energy

Sumas, Washington

AQUILA ENERGY MARKETING CORPORATION

Aquila Canada Corporation

Barret Resources Corporation

Sumas, Washington

AQUILA ENERGY MARKETING CORPORATION

Aquila Canada Corporation

Cargill Energy Division

Port of Morgan, MT

AQUILA ENERGY MARKETING CORPORATION

Aquila Canada Corporation

CoEnergy Trading Company

Detroit, Michigan

AQUILA ENERGY MARKETING CORPORATION

Aquila Canada Corporation

CoEnergy Trading Company

St. Clair, Michigan

AQUILA ENERGY MARKETING CORPORATION

Aquila Canada Corporation

Commonwealth Edison

Port of Morgan, MT

AQUILA ENERGY MARKETING CORPORATION

Aquila Canada Corporation

Cook Inlet

Eastport, Idaho

AQUILA ENERGY MARKETING CORPORATION

Aquila Canada Corporation

Coral Energy Resources

Sumas, Washington

AQUILA ENERGY MARKETING CORPORATION

Aquila Canada Corporation

Duke Energy Trading \& Marketing

Eastport, Idaho

AQUILA ENERGY MARKETING CORPORATION

Aquila Canada Corporation

Duke Energy Trading \& Marketing

St. Clair, Michigan

AQUILA ENERGY MARKETING CORPORATION

Aquila Canada Corporation

Duke Energy Trading \& Marketing

Sumas, Washington

AQUILA ENERGY MARKETING CORPORATION

Aquila Canada Corporation

El Paso Energy Marketing

$\begin{array}{llll} & & & \text { Quarterly } \\ \text { October } & \text { November } & \text { December } & \text { Avg. } \\ \text { Vol. Price Vol. Price Vol. Price } & \text { Vol. Price }\end{array}$

$816 \quad 2.39$

- N/A

$0 \quad$ N/A

816

2.39

$\begin{array}{llll}995 & 2.39 & 108 & 2.92\end{array}$

O N/A

1103

2.44

$0 \quad$ N/A

301

2.37

o N/A

301

2.37

$0 \quad$ N/A

$0 \quad$ N/A

$5 \quad 2.74$

5

2.74

$\begin{array}{llll}0 & \text { N/A } & 132 & 2.30\end{array}$

o N/A

132

2.30

- N/A

o N/A

312

2.09

312

2.09

- N/A

$137 \quad 3.02$

$0 \quad$ N/A

137

3.02

- N/A

886

2.92

908

2.28

1793

2.60

- N/A

$347 \quad 3.02$

712

2.34

1059

2.56

- N/A

322

2.11

$0 \quad$ N/A

322

2.11

o N/A

583

2.92

$0 \quad N / A$

583

2.92

St. Clair, Michigan

197

2.63

o N/A

- N/A

197

2.63 
SHORT-TERM SALES

YEAR: 1999 Quarter: Four

Estimated Volumes (MMCF) \& Prices (\$ / MMBTU)

Importer

Seller

Purchaser / End User

Point

of

Entry

AQUILA ENERGY MARKETING CORPORATION

Aquila Canada Corporation

Enron North America Corp.

Detroit, Michigan

AQUILA ENERGY MARKETING CORPORATION

Aquila Canada Corporation

Enron North America Corp.

St. Clair, Michigan

AQUILA ENERGY MARKETING CORPORATION

Aquila Canada Corporation

Nicor Gas Company

Port of Morgan, MT

AQUILA ENERGY MARKETING CORPORATION

Aquila Canada Corporation

PG\&E Energy Trading

Sumas, Washington

AQUILA ENERGY MARKETING CORPORATION

Aquila Canada Corporation

Portland General Electric Company

Sumas, Washington

AQUILA ENERGY MARKETING CORPORATION

Aquila Canada Corporation

Southem Company Energy

Eastport, idaho

AQUILA ENERGY MARKETING CORPORATION

Aquila Canada Corporation

Southem Company Energy

Sumas, Washington

AQUILA ENERGY MARKETING CORPORATION

Aquila Canada Corporation

Southwest Gas Corporation

Sumas, Washington

AQUILA ENERGY MARKETING CORPORATION

Aquila Canada Corporation

TXU Energy Trading Company

St. Clair, Michigan

1316

October

Vol. Price

November
Vol. Price

December

Vol. Price

Quarterly

Total

Vol. Price

N/A

- N/A

$53 \quad 2.53$

53

2.53

$21 \quad 2.58$

42

2.15

o N/A

63

2.29

598

2.78

$0 \quad$ N/A

- N/A

598

2.78

$0 \quad$ N/A

151

2.92

- N/A

151

2.92

$179 \quad 2.39$

302

2.92

0

N/A

482

2.72

- N/A

374

3.02

0

N/A

374

3.02

- N/A

- N/A

685

2.28

685

2.28

O N/A

130

2.92

N/A

130

2.92

ARCO PRODUCTS COMPANY

CanWest Gas Supply Inc.

Arco Products Company

Sumas, Washington

$10 \quad 2.85$

111

2.16

168

2.38

288

2.31

ARCO PRODUCTS COMPANY

Coral Energy Canada inc.

Arco Products Company

Sumas, Washington

99

2.39

80

2.70

149

2.22

328

2.39

ARCO PRODUCTS COMPANY

Duke Energy Marketing Canada

Arco Products Company

Sumas, Washington
13

2.43

145

2.53

217

2.12

493

2.32 
Seller

Purchaser / End User

SHORT-TERM SALES

YEAR: 1999 Quarter: Four

Estimated Volumes (MMCF) \& Prices (\$ / MMBTU)

ARCO PRODUCTS COMPANY

Engage Energy Canada

Arco Products Company

Sumas, Washington

$434 \quad 2.40$

$385 \quad 2.41$

$374 \quad 2.39$

1193

2.40

ARCO PRODUCTS COMPANY

Enron Capital \& Trade Resources Canada Arco Products Company

Sumas, Washington

$33 \quad 2.64$

$0 \quad$ N/A

$0 \quad$ N/A

$33 \quad 2.64$

AVISTA CORPORATION

Amoco Canada Petroleum Company Ltd.

Avista Corporation

Eastport, Idaho

$0 \quad N / A$

$297 \quad 2.45$

307

2.23

$604 \quad 2.34$

AVISTA CORPORATION

Avista Energy Inc.

Avista Comporation

AVISTA CORPORATION

Engago Energy Canada

Avista Corporation

Eastport, Idaho

$307 \quad 2.31$

AVISTA CORPORATION

Enron Capital \& Trade Resources Canada Avista Corporation

Eastport, Idaho

$307 \quad 2.31$

$297 \quad 2.70$

$307 \quad 2.25$

$911 \quad 2.42$

AVISTA CORPORATION

Petro-Canada Hydrocarbons Inc.

Avista Corporation

Eastport, Idaho

$307 \quad 2.36$

$0 \quad$ N/A

$0 \quad$ N/A

307

2.36

AVISTA ENERGY, INC.

Avista Comporation

Avista Energy

Eastport, Idaho

$12 \quad 2.39$

$379 \quad 2.13$

$86 \quad 2.09$

477

2.13

AVISTA ENERGY, INC.

CanWest Gas Supply Inc.

Avista Energy

Sumas, Washington

$926 \quad 2.40$

$0 \quad$ N/A

$0 \quad$ N/A

926

2.40

AVISTA ENERGY, INC.

Canadian Natural Resources Limited

Avista Energy

Sumas, Washington

$465 \quad 2.40$

o N/A

$0 \quad$ N/A

465

2.40

AVISTA ENERGY, INC.

Coral Energy Canada Inc.

Avista Energy

Sumas, Washington

$513 \quad 2.35$

$0 \quad$ N/A

$0 \quad$ N/A

$513 \quad 2.35$

AVISTA ENERGY, INC.

Husky Oil

Avista Energy

Sumas, Washington

$430 \quad 2.40$

o N/A

$0 \quad$ N/A

$430 \quad 2.40$ 
SHORT-TERM SALES

YEAR: 1999 Quarter: Four

Estimated Volumes (MMCF) \& Prices (\$ / MMBTU)
Importer

Seller

Purchaser/End User

AVISTA ENERGY, INC.

KN Marketing, L.P.

Avista Energy

AVISTA ENERGY, INC

Numac Energy

Avista Energy

AVISTA ENERGY, INC.

PG\&E Energy Trading

Avista Energy

AVISTA ENERGY, INC

Petro-Canada Hydrocarbons Inc.

Avista Energy

AVISTA ENERGY, INC.

Sempra Energy Trading

Avista Energy

BARRINGTON PETROLEUM LTD.

Barrington Petroleum Limited

Enron Capital \& Trade Resources

BC GAS UTILITY LTD.

B.C. Gas Inc.

Coral Energy Resources

BC GAS UTILITY LTD.

B.C. Gas Inc.

Duke Energy Trading \& Marketing

BC GAS UTILITY LTD.

B.C. Gas inc.

Enron Capital \& Trade Resources

BC GAS UTILITY LTD.

B.C. Gas Inc.

Puget Sound Energy

BIG SKY GAS MARKETING

Coral Energy Canada Inc.

Big Sky Gas Marketing

BIG SKY GAS MARKETING

Engage Energy Canada

Big Sky Gas Marketing
Point

of

Sumas, Washington

$128 \quad 2.41$

o N/A

- N/A

128

2.41

Sumas, Washington

$428 \quad 1.80$

- N/A

O N/A

428

1.80

Eastport, Idaho

$873 \quad 1.84$

$830 \quad 2.11$

85

1.98

2561

1.97

Sumas, Washington

$480 \quad 2.39$

- N/A

o N/A

480

2.39

Sumas, Washington

o N/A

3185

Port of Morgan, MT

$152 \quad 2.54$

Sumas, Washington

$0 \quad$ N/A

$\begin{array}{ll}3 & 1.88\end{array}$

$2 \quad 2.28$

$5 \quad 2.06$

Sumas, Washington

$\begin{array}{llll}0 & \text { N/A } & 19 & 1.80\end{array}$

o N/A

19

1.80

Sumas, Washington

$10 \quad 2.85$

$4 \quad 2.05$

144

2.27

157

2.30

Sumas, Washington

$175 \quad 2.56$

$250 \quad 2.21$

290

2.27

714

2.32

Noyes, Minnesota

o N/A

$90 \quad 2.01$

$0 \quad$ N/A

$90 \quad 2.01$

Noyes, Minnesota $\begin{array}{ll}7 & 2.31\end{array}$

38
2.59
$260 \quad 1.95$

651

2.33 
SHORT-TERM SALES

YEAR: 1999 Quarter: Four

Estimated Volumes (MMCF) \& Prices (\$ / MMBTU)

Importer

Seller
Purchaser / End User

Point

Entry

BIG SKY GAS MARKETING

Enron Capital \& Trade Resources Canada

Big Sky Gas Marketing

CABOT OIL \& GAS MARKETING CORPORATION

Avista Energy Inc.

National Fuel Marketing Company, L.L.C.

Sumas, Washington

CABOT OIL \& GAS MARKETING CORPORATION

Boeing Company

Various WA State Markets

Sumas, Washington

CABOT OIL \& GAS MARKETING CORPORATION

Enron Capital \& Trade Resources Canada

IGI Resources

Sumas, Washington

CABOT OIL \& GAS MARKETING CORPORATION

KN Marketing, L.P.

Reliant Energy Services, Inc.

Sumas, Washington

CABOT OIL \& GAS MARKETING CORPORATION

KN Marketing, L.P.

TransCanada Gas Services

Sumas, Washington

CABOT OIL \& GAS MARKETING CORPORATION

Portland General Electric

National Fuel Marketing Company, L.L.C.

Sumas, Washington

O N/A

$0 \quad$ N/A

$4 \quad 2.38$

$4 \quad 2.38$

CANWEST GAS SUPPLY U.S.A., INC.

CanWest Gas Supply Inc.

Avista Energy

Sumas, Washington

$9 \quad 2.43$

- N/A

$0 \quad$ N/A

$9 \quad 2.43$

CANWEST GAS SUPPLY U.S.A., INC.

CanWest Gas Supply inc.

Barret Resources Corporation

Sumas, Washington

$19 \quad 2.43$

626

2.79

369

2.31

1015

2.61

CANWEST GAS SUPPLY U.S.A., INC.

CanWest Gas Supply Inc.

Cascade Natural Gas

Sumas, Washington

- N/A

56

2.79

$0 \quad$ N/A

56

2.79

CANWEST GAS SUPPLY U.S.A., INC.

CanWest Gas Supply Inc.

Coral Energy Resources

Sumas, Washington

o N/A

$43 \quad 2.79$

o N/A

$43 \quad 2.79$

CANWEST GAS SUPPLY U.S.A., INC.

CanWest Gas Supply Inc.

Enron Capital \& Trade Resources
Sumas, Washington $\begin{array}{ll}6 & 2.79\end{array}$

o N/A

$6 \quad 2.79$ 


\section{SHORT-TERM SALES}

YEAR: 1999 Quarter: Four

Estimated Volumes (MMCF) \& Prices (\$ / MMBTU)

Importer

Seller

Purchaser/End User

CANWEST GAS SUPPLY U.S.A., INC.

CanWest Gas Supply Inc.

Enserco Energy, nc.

CANWEST GAS SUPPLY U.S.A., INC

CanWest Gas Supply Inc.

Kimball Energy Comoration

CANWEST GAS SUPPLY U.S.A., INC.

CanWest Gas Supply Inc.

Puget Sound Energy

CANWEST GAS SUPPLY U.S.A., INC.

CanWest Gas Supply Inc.

Questar Energy Trading Co.

CANWEST GAS SUPPLY U.S.A., INC.

CanWest Gas Supply inc.

Sierra Pacific Power Company

CANWEST GAS SUPPLY U.S.A., INC.

CanWest Gas Supply Inc.

Southem Company Energy

CANWEST GAS SUPPLY U.S.A., INC.

CanWest Gas Supply Inc.

Southwest Gas Corporation

CARTHAGE ENERGY SERVICES, INC.

Comerstone Natural Gas Company

Engage Energy U.S. LP

Noyes, Minnesota

O N/A

206

$2 \quad 2.79$

$0 \quad$ N/A

$2 \quad 2.79$

Sumas, Washington

O N/A

$67 \quad 2.79$

$34 \quad 2.31$

101

2.63

Sumas, Washington

o N/A

$\begin{array}{ll}65 & 2.79\end{array}$

$269 \quad 2.31$

$334 \cdot 2.40$

Sumas, Washington

O N/A

$90 \quad 2.79$

101

2.31

191

2.54

CARTHAGE ENERGY SERVICES, INC.

Comerstone Natural Gas Company

Enron Capital \& Trade Resources

Noyes, Minnesota

O N/A

$0 \quad$ N/A

$251 \quad 2.23$

251

CARTHAGE ENERGY SERVICES, INC.

Comerstone Natural Gas Company

TransCanada Gas Services

Noyes, Minnesota

$522 \quad 2.56$

$243 \quad 3.02$

O N/A

765

2.70

CASCADE NATURAL GAS CORPORATION

Amoco Canada Petroleum Company Ltd. Cascade Natural Gas

Sumas, Washington

CASCADE NATURAL GAS CORPORATION

Barrett Resources

Cascade Natural Gas
Sumas, Washington o N/A

- N/A

12.68

1 


\section{SHORT-TERM SALES \\ YEAR: 1999 Quarter: Four \\ Estimated Volumes (MMCF) \& Prices (\$/MMBTU)}

Importer

Seller

Purchaser / End User

CASCADE NATURAL GAS CORPORATION

CanWest Gas Supply Inc.

Cascade Natural Gas

- Sumas, Washington

CASCADE NATURAL GAS CORPORATION

Coast Energy Canada

Cascade Natural Gas

CASCADE NATURAL GAS CORPORATION

E-Prime

Cascade Natural Gas

CASCADE NATURAL GAS CORPORATION Engage Energy Canada

Cascade Natural Gas

CASCADE NATURAL GAS CORPORATION Enron Capital \& Trade Resources Canada

Cascade Natural Gas

CASCADE NATURAL GAS CORPORATION Ensorco Energy Marketing Canada

Cascade Natural Gas

CASCADE NATURAL GAS CORPORATION Kimball Energy Corporation

Cascade Natural Gas

CASCADE NATURAL GAS CORPORATION

Reliant Energy Services Canada, Inc.

Cascade Natural Gas

Point

of

October

Vol. Price

November

Vol. Price

December

Vol. Price

Quarterly

Total

Avg.

Vol. Price

o N/A

$60 \quad 2.41$

$0 \quad$ N/A

$60 \quad 2.41$

Sumas, Washington

$0 \quad$ N/A

$10 \quad 2.62$

- N/A

$10 \quad 2.62$

Sumas, Washington

o N/A

$90 \quad 2.93$

$0 \quad$ N/A

90

2.93

Sumas, Washington

$646 \quad 2.35$

11

2.08

47

2.60

1133

2.45

Sumas, Washington

$310 \quad 2.40$

o N/A

- N/A

310

2.40

Sumas, Washington

$444 \quad 2.39$

o N/A

- N/A

444

2.39

Sumas, Washington

o N/A

o N/A

462

2.69

462

2.69

Sumas, Washington

$0^{\circ} \quad$ N/A

$99 \quad 2.92$

$309 \quad 2.68$

408

2.74

CASCADE NATURAL GAS CORPORATION

Sandia Energy Resources Company

Cascado Natural Gas

Sumas, Washington

o N/A

298

2.84

231

2.20

529

CHEVRON U.S.A. INC. (SP)

Chevron Canada Resources

Dynegy Marketing \& Trade

Eastport, Idaho

$18 \quad 2.61$

$17 \cdot 2.12$

$92 \quad 2.25$

$127 \quad 2.28$

CMS MARKETING SERVICES AND TRADING COMPANY

Northwest Shelf LNG Sellers

Altrade

Amoco Trading
Lake Charles, LA

CMS MAAKETING SERVICES AND TRADING COMPANY Northwest Shelf LNG Sellers

Lake Charles, LA
1342.09
$276 \quad 2.38$
$34 \quad 2.38$

$0 \quad$ N/A

$0 \quad$ N/A

$34 \quad 2.38$

SP Interruptible/special purchase sale made under Long-term authorization. 


\section{SHORT-TERM SALES \\ YEAR: 1999 Quarter: Four \\ Estimated Volumes (MMCF) \& Prices (\$ / MMBTU)}

\begin{tabular}{ll} 
Importer & Point \\
Seller & of \\
Purchaser/End User & Entry \\
\hline
\end{tabular}

CMS MARKETING SERVICES AND TRADING COMPANY

Northwest Shelf LNG Sellers

Columbia Energy Services

Lake Charles, LA

CMS MARKETING SERVICES AND TRADING COMPANY

Northwest Shelf LNG Sellers

Consumers Energy Company

Lake Charles, LA

$387 \quad 2.09$

O N/A

$310 \quad 2.38$

698

2.22

CMS MARKETING SERVICES AND TRADING COMPANY

Northwest Shelf LNG Sellers

Duke Field Services

Lake Charles, LA

$730 \quad 2.09$

$0 \quad$ N/A

$756 \quad 2.38$

$1486 \cdot 2.24$

CMS MARKETING SERVICES AND TRADING COMPANY Northwest Shelf LNG Sellers

Enline Energy Solutions

Lake Charles, LA

O N/A

O N/A

$20 \quad 2.38$

20

2.38

CMS MARKETING SERVICES AND TRADING COMPANY

Northwest Shelf LNG Sellers

Enron Capital \& Trade Resources

Lake Charles, LA

$134 \quad 2.09$

$0 \quad$ N/A

$0 \quad$ N/A

$134 \quad 2.09$

CMS MARKETING SERVICES AND TRADING COMPANY

Northwest Shelf LNG Sellers

KN Energy

Lake Charles, LA

O N/A

$0 \quad$ N/A

$57 \quad 2.38$

$57 \quad 2.38$

CMS MARKETING SERVICES AND TRADING COMPANY Northwest Shelf LNG Sellers

Noble Gas Marketing

Lake Charles, LA

$173 \quad 2.09$

$0 \quad$ N/A

$80 \quad 2.38$

253

2.18

CMS MARKETING SERVICES AND TRADING COMPANY Northwest Shelf LNG Sellers

Oxy, Inc.

Lake Charles, LA

$0^{\cdot} \quad$ N/A.

$0 \quad$ N/A

$267 \quad 2.38$

267

2.38

CMS MARKETING SERVICES AND TRADING COMPANY

Northwest Shelf LNG Sellers

TXU Energy Trading Company

Lake Charles, LA

O N/A

$0 \quad$ N/A

267

2.38

267

2.38

CMS MARKETING SERVICES AND TRADING COMPANY Northwest Shelf LNG Sellers

Tejas Power

Lake Charles, LA

$111 \quad 2.09$

- N/A

N/A

111

2.09

CMS MARKETING SERVICES AND TRADING COMPANY Northwest Shelf LNG Sellers

Torch Gas L.C.

Lake Charles, LA

$442 \quad 2.09$

- N/A

N N/A

442

2.09

CMS MARKETING SERVICES AND TRADING COMPANY Northwest Shelf LNG Sellers

TransCanada Gas Services

Lake Charles, LA
$38 \quad 2.09$

o N/A

$37 \quad 2.38$

75

2.23 
Page - 81

SHORT-TERM SALES

YEAR: 1999 Quarter: Four

Estimated Volumes (MMCF) \& Prices (\$ / MMBTU)

Importer

Seller

Purchaser / End User

Point

Entry

CMS MARKETING SERVICES AND TRADING COMPANY

Qatar Liquefied Gas Co. Ltd.

Altrade

Lake Charies, LA

CMS MARKETING SERVICES AND TRADING COMPANY

Qatar Liquefied Gas Co. Ltd.

Ameren

Lake Charles, LA

$0 \quad$ N/A

$52 \quad 3.07$

- N/A

$52 \quad 3.07$

CMS MARKETING SERVICES AND TRADING COMPANY

Qatar Liquefied Gas Co. Ltd.

Aquila Energy Marketing

Lake Charles, LA

$0 \quad$ N/A

$481 \quad 3.07$

$0 \quad$ N/A

$481 \quad 3.07$

CMS MARKETING SERVICES AND TRADING COMPANY

Qatar Liquefied Gas Co. Ltd.

CMS Gas Marketing

Lake Charles, LA

$0 \quad$ N/A

$534 \quad 3.07$

$0 \quad$ N/A

$534 \quad 3.07$

CMS MARKETING SERVICES AND TRADING COMPANY

Qatar Liquefied Gas Co. Ltd.

Cleco

Lake Charles, LA

CMS MARKETING SERVICES AND TRADING COMPANY

Qatar Liquefied Gas Co. Ltd.

Columbia Energy Services

Lake Charles, LA

CMS MARKETING SERVICES AND TRADING COMPANY

Qatar Liquefied Gas Co. Ltd.

Consumers Energy Company

Lake Charles, LA

CMS MARKETING SERVICES AND TRADING COMPANY

Qatar Liquefied Gas Co. Ltd.

Duke Field Services

Lake Charles, LA

- N/A

311

3.07

$0 \quad$ N/A

311

3.07

CMS MARKETING SERVICES AND TRADING COMPANY

Qatar Liquefied Gas Co. Ltd.

Dynegy Marketing \& Trade

Lake Charies, LA

O N/A

36

3.07

$0 \quad$ N/A

$36 \quad 3.07$

CMS MARKETING SERVICES AND TRADING COMPANY

Qatar Liquefied Gas Co. Ltd.

Enline Energy Solutions

Lake Charles, LA

- N/A

17

3.07

- N/A

$17 \quad 3.07$

CMS MARKETING SERVICES AND TRADING COMPANY

Qatar Liquefied Gas Co. Ltd.

KN Energy

Lake Charles, LA

O N/A

$\begin{array}{ll}68 & 3.07\end{array}$

$0 \quad$ N/A

$68 \quad 3.07$

CMS MARKETING SERVICES AND TRADING COMPANY

Qatar Liquefied Gas Co. Lid.

Noble Gas Marketing

Lake Charles, LA

$\begin{array}{llllllll}0 & \text { N/A } & 19 & 3.07 & 0 & \text { N/A } & 19 & 3.07\end{array}$




\section{SHORT-TERM SALES}

YEAR: 1999 Quarter: Four

Estimated Volumes (MMCF) \& Prices (\$ / MMBTU)

Importer

Seller

Purchaser/End User
October
Vol Price
November

Entry

Vol. Price

CMS MARKETING SERVICES AND TRADING COMPANY

Qatar Liquefied Gas Co. Ltd.

Oxy, Inc.

Lake Charies, LA

- N/A

$50 \quad 3.07$

December

Vol. Price

Quarterily

Total

Avg. Price

Qatar Liquefied Gas Co. Ltd.

Tejas Power

Lake Charles, LA

o N/A

$24 \quad 3.07$

$0 \quad N / A$

$24 \quad 3.07$

CMS MARKETING SERVICES AND TRADING COMPANY

Qatar Liquefied Gas Co. Ltd.

Torch Gas L.C.

Lake Charles, LA

O N/A

$120 \quad 3.07$

O N/A

$120 \quad 3.07$

CMS MARKETING SERVICES AND TRADING COMPANY

Qatar Liquefied Gas Co. Ltd.

TransCanada Gas Services

Lake Charies, LA

$0 \quad$ N/A

$37 \quad 3.07$

$0 \quad$ N/A

$37 \quad 3.07$

CMS MARKETING SERVICES AND TRADING COMPANY

Qatar Liquefied Gas Co. Ltd.

Unocal

Lake Charles, LA

$0 \quad$ N/A

$18 \quad 3.07$

- N/A

$18 \quad 3.07$

CMS MARKETING SERVICES AND TRADING COMPANY

Qatar Liquefied Gas Co. Ltd.

Western Gas Marketing Inc.

Lake Charles, LA

o N/A

$9 \quad 3.07$

o N/A

$9 \quad 3.07$

CMS MARKETING SERVICES AND TRADING COMPANY

Ras Laffan Liquefied Nat. Gas Co.

Ameren

Lake Charles, LA

$0 \quad$ N/A

O N/A

$104 \quad 3.20$

104

3.20

CMS MARKETING SERVICES AND TRADING COMPANY

Ras Laffan Liquefied Nat. Gas Co.

Tejas Power

Lake Charles, LA

O N/A

O N/A

$70 \quad 3.20$

$70 \quad 3.20$

COAST ENERGY GROUP

Coast Energy Canada

Various CA State Markets

Eastport, Idaho

739

2.08

810

2.32

$741 \quad 1.32$

$2289 \quad 1.92$

COAST ENERGY GROUP

Coast Energy Canada

Various WA State Markets

Sumas, Washington

$20 \quad 2.74$

- N/A

$0 \quad$ N/A

20

2.74

COENERGY TRADING COMPANY

Duke Energy Marketing Canada

National Fuel Gas Distribution

Niagara Falls, NY

- N/A

- N/A

155

2.81

$155 \quad 2.81$

COENERGY TRADING COMPANY

Renaissance Energy Ltd.

CoEnergy Trading Company

Niagara Falls, NY

$16 \quad 2.68$

o N/A

o N/A

$16 \quad 2.68$ 


\section{SHORT-TERM SALES}

YEAR: 1999 Quarter: Four

Estimated Volumes (MMCF) \& Prices (\$ / MMBTU)

Importer

Seller

Purchaser / End User

COMMONWEALTH GAS COMPANY

ANE

Commonwealth Gas Company

CORAL ENERGY RESOURCES, L.P.

Coral Energy Canada Inc.

City of Ellensburg, WA

CORAL ENERGY RESOURCES, L.P.

Coral Energy Canada Inc.

Mead Paper

CORAL ENERGY RESOURCES, L.P. Coral Energy Canada Inc.

Michigan Consolidated Gas Company

CORAL ENERGY RESOURCES, L.P. Coral Energy Canada Inc.

Minnesota Gas Company

CORAL ENERGY RESOURCES, L.P.

Coral Energy Canada Inc.

Perry Gas Company

CORAL ENERGY RESOURCES, L.P.

Coral Energy Canada Inc.

Proctor and Gamble Company

CORAL ENERGY RESOURCES, L.P.

Coral Energy Canada Inc.

Various MI State Markets

Noyes, Minnesota

CORAL ENERGY RESOURCES, L.P.

Coral Energy Canada Inc.

Various MN State Markets

Noyes, Minnesota

CORAL ENERGY RESOURCES, L.P.

Coral Energy Canada Inc.

Various NY State Markets

Waddington, NY

CORAL ENERGY RESOURCES, L.P.

Coral Energy Canada Inc.

Wisconsin Public Service Corp.

Noyes, Minnesota

CRESTAR ENERGY MARKETING CORPORATION

Crestar Energy

N.Y. State Electric \& Gas

Niagara Falls, NY
$137 \quad 3.68$
$642 \quad 2.60$

o N/A

o N/A

$642 \quad 2.60$

$0 \quad$ N/A

152

3.11

$1075 \quad 2.03$

1228

2.16

$77 \quad 2.54$

O N/A

$15 \quad 3.01$

$92 \quad 2.62$

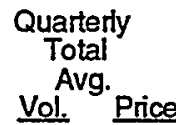

276

1.95

2.60

2.85

2.78

$201 \quad 2.31$

$252 \quad 2.21$

- N/A

- N/A

$252 \quad 2.21$ $\begin{array}{ll}5 & 4.47\end{array}$

- N/A

$142 \quad 3.71$ 


\section{SHORT-TERM SALES}

YEAR: 1999 Quarter: Four

Estimated Volumes (MMCF) \& Prices (\$ / MMBTU)

Importer

Seller

Purchaser/End User

CXY ENERGY MARKETING (U.S.A.) INC.

CXY Marketing

AEP Energy Services

CXY ENERGY MARKETING (U.S.A.) INC.

CXY Marketing

AEP Energy Services

CXY ENERGY MARKETING (U.S.A.) INC.

CXY Marketing

AEP Energy Services

CXY ENERGY MARKETING (U.S.A.) INC.

CXY Marketing

Adams Resources Marketing, Ltd.

CXY ENERGY MARKETING (U.S.A.) INC.

CXY Marketing

Altrade

OXY ENERGY MARKETING (U.S.A.) INC.

CXY Marketing

Altrade

CXY ENERGY MARKETING (U.S.A.) INC.

CXY Marketing

Aquila Energy Marketing

Noyes, Minnesota

Port of Morgan, MT

Eastport, Idaho

$18 \quad 2.41$

o N/A

155

2.10

173

2.13

CXY ENERGY MARKETING (U.S.A.) INC.

CXY Marketing

CMS Gas Marketing

Noyes, Minnesota

155

2.61

o N/A

- N/A

$155 \quad 2.61$

CXY ENERGY MARKETING (U.S.A.) INC.

CXY Marketing

Cibola Corporation

Port of Morgan, MT

185

2.16

135

2.96

$195 \quad 1.89$

$515 \quad 2.27$

CXY ENERGY MARKETING (U.S.A.) INC.

CXY Marketing

Coast Energy Group

$\begin{aligned} & \text { Quarterty } \\ & \text { Total } \\ & \text { Avg. } \\ & \text { Vol. Price }\end{aligned}$

$74 \quad 2.58$

$256 \quad 2.35$

$305 \quad 2.51$

70. 2.30

$25 \quad 2.32$

$46 \quad 2.36$

$483 \quad 0.28$

$52 \quad 2.15$
Eastport, Idaho 
SHORT-TERM SALES

YEAR: 1999 Quarter: Four

Estimated Volumes (MMCF) \& Prices (\$ / MMBTU)
Importer

Seller

Purchaser/End User

CXY ENERGY MARKETING (U.S.A.) INC.

CXY Marketing

Columbia Energy Services

CXY ENERGY MARKETING (U.S.A.) INC.

CXY Marketing

Columbia Energy Services

CXY ENERGY MARKETING (U.S.A.) INC.

CXY Marketing

Coral Energy Resources

CXY ENERGY MARKETING (U.S.A.) INC.

CXY Marketing

Duke Energy Trading \& Marketing

CXY ENERGY MARKETING (U.S.A.) INC.

CXY Marketing

Duke Energy Trading \& Marketing

CXY ENERGY MARKETING (U.S.A.) INC.

CXY Marketing

Duke Energy Trading \& Marketing

CXY ENERGY MARKETING (U.S.A.) INC.

CXY Marketing

Dynegy Marketing \& Trade

CXY ENERGY MARKETING (U.S.A.) INC.

CXY Marketing

El Paso Energy Marketing

CXY ENERGY MARKETING (U.S.A.) INC.

CXY Marketing

El Paso Energy Marketing

CXY ENERGY MARKETING (U.S.A.) INC.

CXY Marketing

Engage Energy U.S. LP

Noyes, Minnesota

$5 \quad 2.47$

$0 \quad$ N/A

o N/A

$5 \quad 2.47$

CXY ENERGY MARKETING (U.S.A.) INC.

CXY Marketing

Enron Capital \& Trade Resources

Noyes, Minnesota

- N/A

$584 \quad 3.26$

o N/A

$584 \quad 3.26$

CXY ENERGY MARKETING (U.S.A.) INC.

CXY Marketing

Enron Capital \& Trade Resources

Point

Noyes, Minnesota

Port of Morgan, MT

Eastport, Idaho

Eastport, Idaho

o. N/A $445 \quad 2.90$

$310 \quad 2.13$

$\begin{array}{ll}755 & 2.58\end{array}$

Noyes, Minnesota

$\begin{array}{llll}0 & \text { N/A } & 25 & 2.68\end{array}$

- N/A

$25 \quad 2.68$

Port of Morgan, MT

$0 \quad$ N/A

0 N/A

$15 \quad 1.80$

$15 \quad 1.80$

Port of Morgan, MT

Niagara Falls, NY

$\begin{array}{llll}0 & \text { N/A } & 190 & 3.33\end{array}$

o N/A

$190 \quad 3.33$

Port of Morgan, MT
$0 \quad$ N/A

o N/A
$180 \quad 1.93$

180

1.93 


\section{SHORT-TERM SALES}

YEAR: 1999 Quarter: Four

Estimated Volumes (MMCF) \& Prices (\$/MMBTU)

Importer

Seller

Purchaser / End User

Point

Entry

Niagara Falls, NY

Waddington, NY

Port of Morgan, MT

Grand Island, NY

Niagara Falls, NY

Niagara Falls, NY

Niagara Falls, NY

Port of Morgan, MT

Noyes, Minnesota

Port of Morgan, MT

$19 \quad 2.16$

27

Eastport, Idaho

$50 \quad 2.83$

0

N/A

o N/A

50

2.83

CXY ENERGY MARKETING (U.S.A.) INC. CXY Marketing

Pancanadian Energy Services
Noyes, Minnesota
Quarterly

Total

Vol. Pvg. Price

Vol. Price

$1 \quad 2.42$

$32 \quad 2.56$

$529 \quad 2.30$

$101 \quad 2.59$

$30 \quad 2.83$

$1431 \quad 2.88$

$29 \quad 2.73$

$305 \quad 2.45$

$17 \quad 2.80$

$459 \quad 2.59$

CXY ENERGY MARKETING (U.S.A.) INC.

CXY Marketing

PGE Gas Transmission NW
$367 \quad 3.21$

373 


\section{SHORT-TERM SALES}

YEAR: 1999 Quarter: Four

Estimated Volumes (MMCF) \& Prices (\$ / MMBTU)

Importer

Seller

Purchaser / End User

CXY ENERGY MARKETING (U.S.A.) INC.

CXY Marketing

Pancanadian Energy Services

CXY ENERGY MAPKETING (U.S.A.) INC.

CXY Marketing

Reliant Energy Services, Inc.

CXY ENERGY MARKETING (U.S.A.) INC.

CXY Marketing

Reliant Energy Services, Inc.

CXY ENERGY MARKETING (U.S.A.) INC.

CXY Marketing

Sempra Energy Trading Corp.

CXY ENERGY MARKETING (U.S.A.) INC.

CXY Marketing

Sierra Pacific Power Company

CXY ENERGY MARKETING (U.S.A.) INC.

CXY Marketing

Southem Company Energy

CXY ENERGY MARKETING (U.S.A.) INC.

CXY Marketing

TXU Energy Trading Company

Port of Morgan, MT

$310 \quad 2.14$

$150 \quad 3.28$

- N/A

460

2.51

Niagara Falls, NY

o N/A

$158 \quad 3.50$

o N/A

158

3.50

Eastport, Idaho

$407 \quad 2.27$

$157 \quad 2.85$

155

2.38

$\begin{array}{ll}719 & 2.42\end{array}$

Port of Morgan, MT

$142 \quad 2.16$

O N/A

- N/A

142

2.16

Grand Island, NY

$21 \quad 3.35$

- N/A

$0 \quad$ N/A

21

3.35

CXY ENERGY MARKETING (U.S.A.) INC.

CXY Marketing

TXU Energy Trading Company

Port of Morgan, MT

- N/A

$139 \quad 3.16$

$152 \quad 1.91$

$291 \quad 2.51$

CXY ENERGY MARKETING (U.S.A.) INC.

CXY Marketing

Tenaska Marketing Ventures

Port of Morgan, MT

$235 \quad 2.00$

O N/A

O N/A

$235 \quad 2.00$

CXY ENERGY MARKETING (U.S.A.) INC.

CXY Marketing

TexEnergy

Waddington, NY

$0 \quad$ N/A

- N/A

$\begin{array}{ll}16 & 2.53\end{array}$

$16 \quad 2.53$

CXY ENERGY MARKETIING (U.S.A.) INC.

CXY Marketing

Texaco Natural Gas

Niagara Falls, NY

$0 \quad$ N/A

$61 \quad 3.64$

311

2.62

$372 \quad 2.79$

CXY ENERGY MARKETING (U.S.A.) INC. CXY Markoting

TransCanada Gas Services
Eastport, Idaho o N/A

o N/A

$33 \quad 2.58$ 
SHORT-TERM SALES

YEAR: 1999 Quarter: Four

Estimated Volumes (MMCF) \& Prices (\$ / MMBTU)
Importer

Seller

Purchaser/End User

Point

Entry

CXY ENERGY MARKETING (U.S.A.) INC.

CXY Marketing

TransCanada Gas Services

CXY ENERGY MARKETING (U.S.A.) INC.

CXY Marketing

Utilicorp United

CXY ENERGY MARKETING (U.S.A.) INC.

CXY Marketing

Williams Energy Company

CXY ENERGY MARKETING (U.S.A.) INC.

CXY Marketing
Wisconsin Gas

DARTMOUTH POWER ASSOCIATES L.P.

Datmouth Power Associates

Duke Energy Trading \& Marketing

DARTMOUTH POWER ASSOCIATES L.P.

Dartmouth Power Associates

Engage Energy U.S. LP

Waddington, NY

Waddington, NY

$63 \quad 3.11$

135

2.56

$32 \quad 2.99$

231

2.77

DARTMOUTH POWER ASSOCIATES L.P.

Dartmouth Power Associates

Southem Company Energy

Waddington, NY

- N/A

O N/A

$93 \quad 2.75$

$93 \quad 2.75$

DEK ENERGY COMPANY

Apache Canada Ltd.

TransCanada Gas Services

Eastport, Idaho

$356 \quad 2.36$

34

1.97

$359 \quad 1.94$

$1062 \quad 2.09$

DISTRIGAS CORPORATION

Enagas

Various MA State Markets

Everett, MA

$1471 \quad 2.09$

$0 \quad$ N/A

o N/A

1471

2.09

DISTRIGAS CORPORATION

Sonatrach

Various MA State Markets

Everett, MA

2928

$$
2.47
$$

1475

2.65

$0 \quad$ N/A

4403

2.53

DUKE ENERGY LNG SALES, INC.

Sonatrading

Enron North America Com.
Lake Charles, LA
$0 \quad$ N/A

1651

2.00

$1651 \quad 2.00$ 


\section{SHORT-TERM SALES \\ YEAR: 1999 Quarter: Four \\ Estimated Volumes (MMCF) \& Prices (\$ / MMBTU)}

Importer

Purchaser / End User

Point

Entry

DUKE ENERGY TRADING AND MKTG SERVICES L.L.C.

Duke Energy Marketing Canada

Duke Energy Trading \& Marketing

Detroit, Michigan

DUKE ENERGY TRADING AND MKTG SERVICES L.L.C.

Duke Energy Marketing Canada

Duke Energy Trading \& Marketing

Eastport, Idaho

DUKE ENERGY TRADING AND MKTG SERVICES L.L.C.

Duke Energy Marketing Canada

Duke Energy Trading \& Marketing

Niagara Falls, NY

$266 \quad 2.67$

$1326 \quad 2.69$

DUKE ENERGY TRADING AND MKTG SERVICES LLC.

Duke Energy Marketing Canada

Duke Energy Trading \& Marketing

Noyes, Minnesota

DUKE ENERGY TRADING AND MKTG SERVICES L.L.C.

Duke Energy Marketing Canada

Duke Energy Trading \& Marketing

Port of Morgan, MT

DUKE ENERGY TRADING AND MKTG SERVICES L.LC.

Duke Energy Marketing Canada

Duke Energy Trading \& Marketing

Sumas, Washington

DUKE ENERGY TRADING AND MKTG SERVICES L.LC.

Duke Energy Marketing Canada

Duke Energy Trading \& Marketing

Waddington, NY

Port of Morgan, MT

$7 \quad 2.48$

Port of Morgan, MT

$25 \quad 2.47$

$0 \quad$ N/A

Port of Morgan, MT

$480 \quad 2.47$

$14 \quad 3.04$

$67 \quad 2.13$

561

2.44

DYNEGY MARKETING \& TRADE

Pan-Alberta Gas Ltd.

Dynegy Inc.

Port of Morgan, MT

$252 \quad 2.58$

123

2.58

85

2.71

1225

2.67

DYNEGY MARKETING \& TRADE

Sempra Energy Trading

Dynegy Inc.

Quarterly

Total

Vol. Price

149

2.15

2026

2.54

$881 \quad 2.73$

$5341 \quad 2.71$

$6957 \quad 2.67$

$6636 \quad 2.46$

$2471 \quad 2.55$

$7 \quad 2.48$

$25 \quad 2.46$

DYNEGYMAAKETING \& TRADE

Nothem Natural Gas
Port of Morgan, MT
$92 \quad 2.47$ $\begin{array}{ll}73 & 2.13\end{array}$

$164 \quad 2.32$ 
SHORT-TERM SALES

YEAR: 1999 Quarter: Four

Estimated Volumes (MMCF) \& Prices (\$ / MMBTU)

Importer

Seller

Purchaser/ End User

EL PASO ENERGY MARKETING COMPANY

El Paso Energy Markeing Canada

El Paso Energy Marketing

EL PASO ENERGY MARKETING COMPANY

El Paso Energy Markeing Canada

El Paso Energy Marketing

ENERGY WEST RESOURCES, INC.

Coral Energy Canada Inc.

Energy West lnc.

ENERGY WEST RESOURCES, INC.

Energy West Resources, Inc.

Various MT State Markets

ENGAGE ENERGY CANADA, L.P.

Engage Energy Canada

Amoco Trading

Sumas, Washington

$0 \quad N / A$

$0 \quad$ N/A

14

2.03

$14 \quad 2.03$

ENGAGE ENERGY CANADA, L.P.

Engage Energy Canada

Barret Resources Corporation

Whitlash, Montana

$32 \quad 2.45$

O N/A

- N/A

$32 \quad 2.45$

ENGAGE ENERGY CANADA, L.P.

Engage Energy Canada

City of Ellensburg, WA

Sumas, Washington

$29 \quad 2.28$

- N/A

O N/A

$29 \quad 2.28$

ENGAGE ENERGY CANADA, L.P.

Engage Energy Canada

Enron North America Corp.

ENGAGE ENERGY CANADA, L.P. Engage Energy Canada

Sumas, Washington

$17 \quad 2.41$

$0 \quad N / A$

$\begin{array}{ll}17 & 2.27\end{array}$

$34 \quad 2.34$

ENGAGE ENERGY CANADA, L.P.

Engage Energy Canada

Intalco Aluminum Corporation

Sumas, Washington

$82 \quad 2.43$

85

2.92

96

2.31

264

2.54

ENGAGE ENERGY CANADA, L.P.

Engage Energy Canada

Kimball Energy Corporation

Sumas, Washington

$21 \quad 2.38$

$8 \quad 2.93$

33

2.27

62

2.39

ENGAGE ENERGY CANADA, L.P.

Engage Energy Canada

Northwest Alloys
Sumas, Washington

$85 \quad 2.43$

83

2.95

85

2.31

253

2.56 


\section{SHORT-TERM SALES \\ YEAR: 1999 Quarter: Four Estimated Volumes (MMCF) \& Prices (\$ / MMBTU)}

Importer

Seller

Purchaser / End User

ENGAGE ENERGY CANADA, L.P. Engage Energy Canada

Oregon Steol Mills

ENGAGE ENERGY CANADA, L.P. Engage Energy Canada

Portand General Electric Company

ENGAGE ENERGY CANADA, L.P. Engage Energy Canada

Potsdam College

ENGAGE ENERGY CANADA, L.P. Engage Energy Canada

Puget Sound Energy

ENGAGE ENERGY CANADA, L.P.

\section{Engage Energy Canada}

Southwest Gas Corporation

ENGAGE ENERGY CANADA, L.P. Engage Energy Canada

St. Lawrence University

ENGAGE ENERGY CANADA, L.P. Engage Energy Canada Tesoro Northwest Company

ENGAGE ENERGY CANADA, L.P. Engage Energy Canada

Tosco Refining Company

ENGAGE ENERGY CANADA, L.P.

Engage Energy Canada

Various WA State Markets

ENGAGE ENERGY U.S., L.P. Engage Energy Canada

Engage Energy U.S. LP

ENGAGE ENERGY U.S., L.P.

Engage Energy Canada

Engage Energy U.S. LP

ENGAGE ENERGY U.S., L.P.

Engage Energy Canada

Engage Energy U.S. L.P
Point

Entry

Sumas, Washington

Sumas, Washington

Massena, New York

Sumas, Washington

Sumas, Washington

Massena, New York

$12 \quad 1.89$

- N/A

$0 \quad$ N/A

$12 \quad 1.89$

Sumas, Washington

Sumas, Washington

Sumas, Washington

Eastport, Idaho

Grand Island, NY

Niagara Falls, NY

October

Vol. Price

November

Vol. Price

December

Vol. Price

Quarterly

Total

Vol. Price

$30 \quad 2.26$

$57 \quad 2.61$

$59 \quad 2.29$

146

2.41

$881 \quad 1.67$

$583 \quad 2.42$

$584 \quad 2.34$

$2049 \quad 2.07$

$17 \quad 1.86$

$\begin{array}{ll}15 & 2.78\end{array}$

$16 \quad 2.78$

$49 \quad 2.45$

o N/A

$37 \quad 1.90$

$260 \quad 2.19$

297

2.15

o N/A

142

2.91

$276 \quad 2.53$

$418 \quad 2.66$

$130 \quad 2.58$

356

2.66

$406 \quad 2.42$

$892 \quad 2.54$

$\begin{array}{ll}69 & 2.57\end{array}$

$94 \quad 2.63$

$120 \quad 2.55$

283

2.58

$570 \quad 2.39$

600

2.92

$525 \quad 2.28$

$1695 \quad 2.54$

$898 \quad 2.14$

96

2.5

$654 \quad 2.48$

$2518 \quad 2.37$

$0 \quad N / A$

48

3.16

23

2.41

$280 \quad 2.54$ 


\section{SHORT-TERM SALES \\ YEAR: 1999 Quarter: Four \\ Estimated Volumes (MMCF) \& Prices (\$ / MMBTU)}

\begin{tabular}{ll} 
Importer & Point \\
Seller & of \\
Purchaser/End User & Entry \\
\hline
\end{tabular}

ENGAGE ENERGY U.S., L.P.

Engage Energy Canada

Engage Energy U.S. LP

ENGAGE ENERGY U.S., L.P.

Engage Energy Canada

Engage Energy U.S. LP

ENGAGE ENERGY U.S., L.P.

Engage Energy Canada

Engage Energy U.S. LP

ENGAGE ENERGY U.S., L.P.

Engage Energy Canada

ENGAGE ENERGY U.S., L.P.

Engage Energy Canada

Vanious NV State Markets

Noyes, Minnesota

October
Vol. Price

November

Vol. Price

December

Vol. Price

Quarterly

Total

Vol. ${ }^{\text {Avg. }}$ Price

Port of Morgan, MT

3307

2.79

$3312 \quad 2.64$

3496

2.26

$10115 \quad 2.56$

St. Clair, Michigan

$\begin{array}{llll}0 & \text { N/A } & 1498 & 3.35\end{array}$

$664 \quad 2.24$

$2163 \quad 3.01$

Waddington, NY

$317 \quad 2.76$

$306 \quad 3.42$

$314 \quad 2.57$

$937 \quad 2.91$

Sumas, Washington

$310 \quad 2.43$

$42 \quad 1.86$

15

2.28

507

2.34

ENGAGE ENERGY U.S., L.P.

Engage Energy Canada

Vanious OR State Markets

Sumas, Washington

ENRON CAPITAL \& TRADE RESOURCES CORP.

B.C. Gas Inc.

Enron Capital \& Trade Resources

Eastport, Idaho

ENRON CAPITAL \& TRADE RESOURCES CORP.

B.C. Gas Inc.

Enron Capital \& Trade Resources

Sumas, Washington

$10 \quad 2.85$

$7 \quad 2.05$

152

2.27

169

2.30

ENRON CAPITAL \& TRADE RESOURCES CORP.

Enron Capital \& Trade Resources Canada

Enron Capital \& Trade Resources

Eastport, Idaho

$3075 \quad 2.53$

$2712 \quad 2.97$

$2781 \quad 2.31$

8568

2.60

ENRON CAPITAL \& TRADE RESOURCES CORP.

Enron Capital \& Trade Resources Canada

Enron Capital \& Trade Resources

Grand Island, NY

$13 \quad 2.60$

O N/A

$4 \quad 2.30$

$17 \quad 2.53$

ENRON CAPITAL \& TRADE RESOURCES CORP.

Enron Capital \& Trade Resources Canada

Enron Capital \& Trade Resources

Niagara Falls, NY

$624 \quad 2.65$

$624 \quad 3.91$

$4 \quad 2.23$

1253

3.28

ENRON CAPITAL \& TRADE RESOURCES CORP.

Enron Capital \& Trade Resources Canada

Enron Capital \& Trade Resources

Noyes, Minnesota
$347 \quad 2.23$

$0 \quad$ N/A 


\section{SHORT-TERM SALES \\ YEAR: 1999 Quarter: Four \\ Estimated Volumes (MMCF) \& Prices (\$ /MMBTU)}

Importer

Seller

Purchaser/End User

Point

Entry

ENRON CAPITAL \& TRADE RESOURCES CORP.

Enron Capital \& Trade Resources Canada

Enron Capital \& Trade Resources

Port of Morgan, MT

ENRON CAPITAL \& TRADE RESOURCES CORP.

Enron Capital \& Trade Resources Canada

Enron Capital \& Trade Resources

St. Clair, Michigan

ENRON CAPITAL \& TRADE RESOURCES CORP.

Enron Capital \& Trade Resources Canada

Enron Capital \& Trade Resources

Sumas, Washington

$347 \quad 2.23$

$658 \quad 2.49$

$25 \quad 2.15$

1031

2.39

ENRON CAPITAL \& TRADE RESOURCES CORP.

Enron Capital \& Trade Resources Canada

Enron Capital \& Trade Resources

Waddington, NY

$1968 \quad 2.73$

$1514 \quad 3.45$

1565

2.59

$5048 \quad 2.90$

ENRON ENERGY SERVICES, INC.

CXY Marketing

Various NY State Markets

Grand Island, NY

$0 \quad$ N/A

$38 \quad 3.50$

18

2.40

56

3.15

ENRON ENERGY SERVICES, INC.

CoEnergy Trading Company

Various NY State Markets

Grand Island, NY

- N/A

$8 \quad 3.50$

$22 \quad 2.50$

30

2.75

ENRON ENERGY SERVICES, INC.

Coral Energy Canada Inc.

Various NY State Markets

Grand Island, NY

o N/A

$0 \quad$ N/A

16

2.30

161

2.30

ENRON ENERGY SERVICES, INC.

Enron Capital \& Trade Resources Canada

Various NY State Markets

Grand Island, NY

$62 \quad 2.70$

$8 \quad 3.47$

ENRON ENERGY SERVICES, INC.

National Fuel Gas Distribution Canada Corp.

Various NY State Markets

Grand Island, NY

O N/A

75

3.49

$78 \quad 2.49$

153

2.98

ENRON ENERGY SERVICES, INC.

Texaco Natural Gas Inc.

Various NY State Markets

Grand Island, NY

$62 \quad 2.65$

o N/A

$0 \quad$ N/A

$62 \quad 2.65$

ENRON INTERNATIONAL GAS SALES COMPANY

Abu Dhabi Gas Liquefaction Co., Ltd.

Enron Intemational Gas Sales Co.

Lake Charles, LA

$0 \quad$ N/A

2713

2.69

$0 \quad N / A$

2713

2.69

H.Q. ENERGY SERVICES (U.S.) INC.

MEHO

H.Q. Energy Services (U.S.) Inc.
Pittsburg, NH
$465 \quad 2.45$

450
465 


\section{SHORT-TERM SALES \\ YEAR: 1999 Quarter: Four \\ Estimated Volumes (MMCF) \& Prices (\$ / MMBTU)}

\begin{abstract}
Importer
Seller

Purchaser/End User

HESS ENERGY SERVICES COMPANY, LLC

Gulf Canada Resources Limited

Nicor Gas Company

HESS ENERGY SERVICES COMPANY, LLC

Gulf Canada Resources Limited

Pancanadian Energy Services
\end{abstract}

Point

of

Entry

HUSKY GAS MARKETING, INC.

Husky Oil

Avista Energy

Eastport, Idaho

October

Vol. Price

November

Vol. Price

Port of Morgan, MT

Port of Morgan, MT

$334 \quad 2.27$

326

2.76

337

December

Vol. Price

Quarterly
Total

Vol. ${ }^{\text {Avg. }}$ Price

2.76

925

2.10

2745

2.37

Noyes, Minnesota

O N/A

1113.02

$115 \quad 2.39$

226

2.70

HUSKY GAS MARKETING, INC.

Husky Oil

Cibola Corporation

Port of Morgan, MT

$481 \quad 2.86$

$149 \quad 3.43$

250

2.07

880

2.73

HUSKY GAS MARKETING, INC.

Husky Oil

Dynegy Marketing \& Trade

Eastport, Idaho

$0 \quad$ N/A

$94 \quad 4.42$

98

3.35

192

3.88

HUSKY GAS MARKETING, INC.

Husky Oil

Engage Energy U.S. LP

Port of Morgan, MT

Sumas, Washington

Port of Morgan, MT

$0 \quad$ N/A

$1 \quad 3.07$

o N/A

13.07

HUSKY GAS MARKETING, INC.

Husky Oil

Koch Energy Trading

Port of Morgan, MT

$119 \quad 2.88$

2.88

110

3.67

$177 \quad 2.95$

o N/A

$0 \quad$ N/A

177

2.95

HUSKY GAS MARKETING, INC

Husky Oil

MidAmerican Energy Company
Port of Morgan, MT

Port of Morgan, MT
- N/A

113

3.70

142

2.02

$255 \quad 2.76$ 


\section{SHORT-TERM SALES}

YEAR: 1999 Quarter: Four

Estimated Volumes (MMCF) \& Prices (\$ / MMBTU)

Importer

Seller

Purchaser / End User

HUSKY GAS MARKETING, INC.

Husky Oil

Minnegasco

HUSKY GAS MARKETING, INC.

Husky Oil

Montana Power

HUSKY GAS MARKETING, INC. Husky Oil

Northem States Power Company

HUSKY GAS MARKETING, INC. Husky Oil

Northem States Power Company

HUSKY GAS MARKETING, INC.

Husky Oil

Northshore Gas Company

HUSKY GAS MARKETING, INC. Husky Oil

OGE Energy Resources

HUSKY GAS MARKETING, INC.

Husky Oil

PG\&E Energy Trading

HUSKY GAS MARKETING, INC. Husky Oil

Peoples Gas, Light, \& Coke

HUSKY GAS MARKETING, INC.

Husky Oil

Phoenix Chemical

HUSKY GAS MARKETING, INC.

Husky Oil

Puget Sound Energy

HUSKY GAS MARKETING, INC.

Husky Oil

San Diego Gas \& Electric Company

HUSKY GAS MARKETING, INC.

Husky Oil

Tenaska Marketing Ventures
Point

Entry

Noyes, Minnesota

Port of Morgan, MT

Noyes, Minnesota

Port of Morgan, MT

Port of Morgan, MT

Port of Morgan, MT

Port of Morgan, MT

Port of Morgan, MT

$240 \quad 2.98$

556

3.70

703

2.05

$1498 \quad 2.81$

Port of Morgan, MT

$119 \quad 2.87$

111

3.70

139

2.02

$369 \quad 2.80$

Sumas, Washington

$39 \quad 2.39$

- N/A

$0 \quad$ N/A

39
2.39

Eastport, ldaho

- N/A

- N/A

$7 \quad 3.54$

$\begin{array}{ll}7 & 3.54\end{array}$

Port of Morgan, MT
$140 \quad 2.91$
$243 \quad 2.12$

$534 \quad 2.65$ 


\section{SHORT-TERM SALES \\ YEAR: 1999 Quarter: Four \\ Estimated Volumes (MMCF) \& Prices (\$/MMBTU)}

Importer

Seller

Purchaser / End User

HUSKY GAS MARKETING, INC.

Husky Oil

U.S. Energy Services

HUSKY GAS MARKETING, INC.

Husky Oil

Wisconsin Power \& Light

Port of Morgan, MT

Sumas, Washington

Sumas, Washington

Sumas, Washington

Sumas, Washington

Sumas, Washington

$17 \quad 2.43$

o N/A

- N/A

$17 \quad 2.43$

IGI RESOURCES, INC.

CanWest Gas Supply inc.

Puget Sound Energy

IGI RESOURCES, INC.

CanWest Gas Supply Inc.

Unocal

Sumas, Washington

$3 \quad 2.43$

- N/A

- N/A

$3 \quad 2.43$

IGI RESOURCES, INC.

CanWest Gas Supply inc. WP Natural Gas

Sumas, Washington

$2 \quad 2.43$

- N/A

- N/A

$2 \quad 2.43$

IGI RESOURCES, INC.

CanWest Gas Supply inc. Weyerhauser

Sumas, Washington

Sumas, Washington

IGI RESOURCES, INC.

CanWest Gas Supply inc.

Willamette

$3 \quad 2.43$

o N/A

$0 \quad$ N/A

$3 \quad 2.43$ 


\section{SHORT-TERM SALES \\ YEAR: 1999 Quarter: Four \\ Estimated Volumes (MMCF) \& Prices (\$ / MMBTU)}

Importer

Seller

Purchaser/End User

IGI RESOURCES, INC.

Duke Energy Marketing Canada Avista Corporation

IGI RESOURCES, INC.

Duke Energy Marketing Canada Boeing Company

IGI RESOURCES, INC.

Duke Energy Marketing Canada

Cascade Natural Gas

IGI RESOURCES, INC.

Duke Energy Marketing Canada Intermountain Gas Company

IGI RESOURCES, INC.

Duke Energy Marketing Canada Intermountain Gas Company

IGI RESOURCES, INC.

Duke Energy Marketing Canada Northwest Natural Gas Company

IGI RESOURCES, INC.

Duke Energy Marketing Canada Puget Sound Energy

IGI RESOURCES, INC.

Duke Energy Marketing Canada Unocal

IGI RESOURCES, INC.

Duke Energy Marketing Canada WP Natural Gas

IGI RESOURCES, INC.

Duke Energy Marketing Canada Weyerhauser

IGI RESOURCES, INC.

Duke Energy Marketing Canada Willamette

Sumas, Washington

$195 \quad 2.43$

$21 \quad 2.95$

$0 \quad$ N/A

$216 \quad 2.48$

IGI RESOURCES, INC.

Engage Energy Canada

Avista Corporation

Point

Entry

Sumas, Washington

Sumas, Washington

Sumas, Washington

Eastport, Idaho

Sumas, Washington

Sumas, Washington

Sumas, Washington

Sumas, Washington

Sumas, Washington

Sumas, Washington

October. November

$\begin{array}{llll}210 & 2.43 & 74 & 2.95\end{array}$

$\begin{array}{ll}71 & 2.31\end{array}$

$355 \quad 2.51$

$0 \quad$ N/A

$152 \quad 2.95$

161

2.31

313

2.62

$817 \quad 2.43$

$853 \quad 2.95$

848

2.31

2518

2.57

$335 \quad 2.31$

$547 \quad 2.70$

72

2.25

1604

2.42

$661 \quad 2.43$

$343 \quad 2.95$

426

2.31

1430

2.52

$1010 \quad 2.43$

$508 \quad 2.95$

613

2.31

2131

2.52

$603 \quad 2.43$

230

2.95

270

2.31

1103

2.51

$170 \quad 2.43$

$8 \quad 2.95$

23

2.31

201

2.44

$149 \quad 2.43$

$61 \quad 2.95$

58

2.31

268

2.52

Sumas, Washington
$31 \quad 2.95$

29

2.31
$125 \quad 2.53$ 
Importer

Purchaser/End User

IGI RESOURCES, INC

Engage Energy Canada

Boeing Company

IGI RESOURCES, INC.

Engage Energy Canada

Cascade Natural Gas

IGI RESOURCES, INC.

Engage Energy Canada

Intermountain Gas Company

IGI RESOURCES, INC.

Engage Energy Canada

Northwest Natural Gas Company

IGI RESOURCES, INC.

Engage Energy Canada

Puget Sound Energy

IGI RESOURCES, INC.

Engage Energy Canada

Unocal

IGI RESOURCES, INC.

Engage Energy Canada

WP Natural Gas

IGI RESOURCES, INC.

Engage Energy Canada

Weyerhauser

Sumas, Washington

$57 \quad 2.43$

$70 \quad 2.95$

$12 \quad 2.31$

$139 \quad 2.68$

IGI RESOURCES, INC.

Engage Energy Canada

Willamette

Sumas, Washington

$61 \quad 2.43$

$9 \quad 2.95$

$0 \quad$ N/A

$70 \quad 2.50$

IGI RESOURCES, INC.

Petro-Canada Hydrocarbons Inc. Avista Corporation

IGI RESOURCES, INC.

Petro-Canada Hydrocarbons Inc. Cascade Natural Gas

Sumas, Washington

$98 \quad 2.43$

- N/A

$0 \quad$ N/A

$98 \quad 2.43$

IGI RESOURCES, INC.

Petro-Canada Hydrocarbons inc.

Intermountain Gas Company 


\section{SHORT-TERM SALES \\ YEAR: 1999 Quarter: Four \\ Estimated Volumes (MMCF) \& Prices (\$ / MMBTU)}

Importer

Seller

Purchaser/End User

IGI RESOURCES, INC.

Petro-Canada Hydrocarbons Inc.

Northwest Natural Gas Company

IGI RESOURCES, INC.

Petro-Canada Hydrocarbons Inc.

Puget Sound Energy

[G] RESOURCES, INC.

Potro-Canada Hydrocarbons Inc. Unocal

Sumas, Washington

$21 \quad 2.43$

$0 \quad$ N/A

$0 \quad$ N/A

$21 \quad 2.43$

IGI RESOURCES, INC.

Petro-Canada Hydrocarbons Inc. WP Natural Gas

Sumas, Washington

$18 \quad 2.43$

$0 \quad$ N/A

$0 \quad$ N/A

$18 \quad 2.43$

IGI RESOURCES, INC.

Petro-Canada Hydrocarbons Inc. Weyemauser

Sumas, Washington

$22 \quad 2.43$

o N/A

$0 \quad$ N/A

22

IGI RESOURCES, INC.

Petro-Canada Hydrocarbons Inc. Willamette

Sumas, Washington

$23 \quad 2.43$

o N/A

O N/A

23

2.43

IGI RESOURCES, INC.

Poco Petroleum Ltd.

Avista Compration

IGI RESOURCES, INC.

Poco Petroleum Ltd.

Boeing Company

IGI RESOURCES, INC.

Poco Petroleum Lid.

Cascade Natural Gas

IGI RESOURCES, INC.

Poco Petroleum Ltd.

Intermountain Gas Company

- N/A

131

$161 \quad 2.31$

$292 \quad 2.60$

IGI RESOURCES, INC.

Poco Petroleum Ltd.

Northwest Natural Gas Company

IGI RESOURCES, INC.

Poco Petroleum Ltd.

Puget Sound Energy
Sumas, Washington

Sumas, Washington

Sumas, Washington

Sumas, Washington

Sumas, Washington

Sumas, Washington
- N/A

194

2.95

$232 \quad 2.31$

426

2.60

$0 \quad$ N/A

$88 \quad 2.95$
Quarterly

Avg.

Vol. Price

$22 \quad 2.43$

$73 \quad 2.43$

$55 \quad 2.64$

$119 \quad 2.62$

$47 \quad 2.63$ 


\section{SHORT-TERM SALES \\ YEAR: 1999 Quarter: Four \\ Estimated Volumes (MMCF) \& Prices (\$ / MMBTU)}

Importer

Seller

Purchaser/End User

IGI RESOURCES, INC.

Poco Petroloum Ltd.

Unocal

IGI RESOURCES, INC.

Poco Petroleum Ltd.

WP Natural Gas

IGI RESOURCES, INC.

Poco Petroleum Ltd.

Weyerhauser

IGI RESOURCES, INC.

Poco Petroleum Lid.

Willamette

IGI RESOURCES, INC.

Talisman Energy, Inc.

Avista Corporation

IGI RESOURCES, INC.

Talisman Energy, Inc.

Cascade Natural Gas

IGI RESOURCES, INC.

Talisman Energy, Inc.

Intermountain Gas Company

IGI RESOURCES, INC.

Talisman Energy, Inc.

Northwest Natural Gas Company

IGI RESOURCES, INC.

Talisman Energy, Inc.

Puget Sound Energy

IGI RESOURCES, INC.

Talisman Energy, Inc.

Unocal

IGI RESOURCES, INC.

Talisman Energy, Inc.

WP Natural Gas

IGI RESOURCES, INC.

Talisman Energy, Inc.

Weyerhauser
Point

Entry

Sumas, Washington

Sumas, Washington

Sumas, Washington

Sumas, Washington

Sumas, Washington

Sumas, Washington

$\begin{array}{ll}73 & 2.43\end{array}$

$0 \quad$ N/A

$0 \quad$ N/A

$73 \quad 2.43$

Sumas, Washington

Sumas, Washington

Sumas, Washington

Sumas, Washington

$15 \quad 2.43$

- N/A

o N/A

$15 \quad 2.43$

Sumas, Washington

$13 \quad 2.43$

o N/A

$0 \quad N / A$

$13 \quad 2.43$

Sumas, Washington
$16 \cdot 2.43$

- N/A

$0 \quad$ N/A

$16 \quad 2.43$ 


\section{SHORT-TERM SALES \\ YEAR: 1999 Quarter: Four \\ Estimated Volumes (MMCF) \& Prices (\$ / MMBTU)}

Importer

Seller

Purchaser/End User

IGI RESOUACES, INC.

Talisman Energy, Inc.

Willamette

INDECK-OSWEGO, L.P.

Aquila Canada Corporation

Indeck Energy Services of Oswego

INDECK-OSWEGO, L.P.

CXY Marketing

indeck Energy Services of Oswego

INDECK-OSWEGO, L.P.

Engage Energy Canada

indeck Energy Services of Oswego

INDECK-OSWEGO, L.P.

Enron Capital \& Trade Resources Canada

Indeck Energy Services of Oswego

INDECK-YERKES, L.P.

Aquila Canada Corporation

Indeck Energy Services of Yerkes

INDECK-YERKES, L.P

CXY Marketing

Indeck Energy Services of Yerkes

INDECK-YERKES, L.P

Direct Energy Marketing Limited

Indeck Energy Services of Yerkes

INDECK-YERKES, L.P.

Enron Capital \& Trade Resources Canada Indeck Energy Services of Yerkes

INDECK-YERKES, L.P.

Northstar Energy Corporation

Indeck Energy Services of Yerkes

INTERENERGY CORPORATION

CEG Energy Options Inc.

Interenergy Corporation

INTERENERGY CORPORATION

TransCanada Gas Services Limited

Interenergy Corporation
Point

Entry

Sumas, Washington

Niagara Falls, NY

Niagara Falls, NY

Niagara Falls, NY

$3 \quad 3.65$

$181 \quad 3.74$

o N/A

185

3.74

Niagara Falls, NY

$297 \quad 3.72$

$1 \quad 3.38$

$150 \quad 3.01$

$449 \quad 3.48$

Niagara Falls, NY

o N/A

$196 \quad 3.84$

$97 \quad 3.01$

$293 \quad 3.56$

Niagara Falls, NY

- N/A

o N/A

$45 \quad 2.89$

45

2.89

Niagara Falls, NY

$203 \quad 3.24$

- N/A

o N/A

203

3.24

Niagara Falls, NY

- N/A

o N/A

$58 \quad 2.84$

$58 \quad 2.84$

Niagara Falls, NY

$170 \quad 2.43$

16

2.47

$172 \quad 2.42$

508

2.44

Portal, ND.

$128 \quad 1.90$

- N/A

o N/A

128

1.90

Portal, ND
$161 \quad 2.33$ o N/A

161

2.33 
SHORT-TERM SALES

YEAR: 1999 Quarter: Four Estimated Volumes (MMCF) \& Prices (\$ / MMBTU)
Importer

Seller

Purchaser/ End User

MONTANA-DAKOTA UTILITIES CO.

Tenaska Marketing Canada

Montana-Dakota Utilities Co.

MURPHY GAS GATHERING

Murphy Oil Company Ltd.

Murphy Gas Gathering

NATIONAL FUEL GAS DISTRIBUTION

CXY Marketing

Various NY State Markets

NATIONAL FUEL GAS DISTRIBUTION

Enron Capital \& Trade Resources Canada

Various NY State Markets

NATIONAL FUEL GAS DISTRIBUTION

Enron Gas Marketing Canada

Various NY Stato Markets

NATIONAL FUEL GAS DISTRIBUTION

Ocean State Power

Various NY State Markets

NATIONAL FUEL GAS DISTRIBUTION TransCanada Gas Services Limited Various NY State Markets

NATIONAL FUEL RESOURCES, INC.

Amoco Canada Petroleum Company Ltd.

Various NY State Markets

Niagara Falls, NY

NATIONAL FUEL RESOURCES, INC.

CXY Marketing

Various NY State Markets

Grand Island, NY

- N/A

$59 \quad 3.51$

$61 \quad 2.53$

120

3.01

NATIONAL FUEL RESOURCES, INC.

CXY Marketing

Various NY State Markets

Niagara Falls, NY

3.28

$171 \quad 2.50$

823

2.78

NATIONAL FUEL RESOURCES, INC.

Coral Energy Canada Inc.

Various NY State Markets

Niagara Falls, NY

$6 \quad 3.07$

- N/A

$0 \quad$ N/A

$6 \quad 3.07$

NATIONAL FUEL RESOURCES, INC.

Duke Energy Marketing Canada

Various NY State Markets

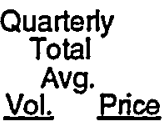

941

2.54

2.18

2.61

2.54

3.01

3.19

2.78

2.50
Niagara Falls, NY 


\section{SHORT-TERM SALES \\ YEAR: 1999 Quarter: Four \\ Estimated Volumes (MMCF) \& Prices (\$ / MMBTU)}

Importer

Seller

Purchaser/End User

NATIONAL FUEL RESOURCES, INC.

Engage Energy Canada

Vanous NY State Markets

NATIONAL FUEL RESOURCES, INC.

Engage Energy Canada

Various NY State Markets

NATIONAL FUEL RESOURCES, INC.

Medina Power

Various NY State Markets

NATIONAL FUEL RESOURCES, INC.

Ocean State Power

Various NY State Markets

Niagara Falls, NY

$\begin{array}{ll}7 & 3.19\end{array}$

$0 \quad$ N/A

$0 \quad$ N/A

$7 \quad 3.19$

NATIONAL FUEL RESOURCES, INC.

Renaissance Energy Ltd.

Various NY State Markets

NATIONAL FUEL RESOURCES, INC.

Renaissance Energy Ltd.

Various NY State Markets

Niagara Falls, NY

$366 \quad 2.72$

$50 \quad 2.22$

$38 \quad 2.46$

455

2.64

NATIONAL FUEL RESOURCES, INC.

Tractebell

Various NY State Markets

Niagara Falls, NY

- N/A

148

3.47

153

2.60

301

3.03

NATIONAL FUEL RESOURCES, INC.

TransCanada Gas Services Limited

Various NY State Markets

Grand Island, NY

$1 \quad 2.87$

0. N/A

$0 \quad$ N/A

1

2.87

NATIONAL FUEL RESOURCES, INC.

TransCanada Gas Services Limited

Various NY State Markets

Niagara Falls, NY

$336 \quad 2.80$

$39 \quad 2.42$

2.40

394

2.74

NEW YORK STATE ELECTRIC \& GAS CORPORATION

Coral Energy Canada inc.

Various NY State Markets

Champlain, NY

O N/A

O N/A

$5 \quad 2.50$

$5 \quad 2.50$

NORTH AMERICAN ENERGY, INC.

Various Suppliers

North American Energy Inc.

Grand Island, NY

$3 \quad 2.69$

$15 \quad 3.47$

$16 \quad 2.27$

$34 \quad 2.85$

NORTH AMERICAN ENERGY, INC.

Various Suppliers

North American Energy Inc. $\begin{array}{ll}73 & 2.70\end{array}$

$57 \quad 3.38$

$70 \quad 2.66$

200

2.88 


\section{SHORT-TERM SALES \\ YEAR: 1999 Quarter: Four \\ Estimated Volumes (MMCF) \& Prices (\$/MMBTU)}

Importer

Seller

Purchaser / End User

NORTHEAST GAS MARKETS L.L.C.

AEC Marketing

New Jersey Natural Gas

NORTHEAST GAS MARKETS LLC.

Amoco Canada Petroleum Company Ltd.

Consolidated Edison

NORTHEAST GAS MARKETS LLC.

Amoco Canada Petroleum Company Ltd.

Long Island Lighting Company

NORTHEAST GAS MARKETS L.L.C.

Amoco Canada Petroleum Company Litd.

N.Y. State Electric \& Gas

Waddington, NY

- N/A

$6 \quad 2.23$

- N/A

$6 \quad 2.23$

NORTHEAST GAS MARKETS L.LC.

Amoco Canada Petroleum Company Ltd. Southem Connecticut Gas Company

NORTHEAST GAS MARKETS L.L.C.

Enron Capital \& Trade Resources Canada Central Hudson Gas \& Electric

NORTHEAST GAS MARKETS LLC.

Enron Capital \& Trade Resources Canada Consolidated Edison

Waddington, NY

O N/A

$33 \quad 2.44$

$0 \quad$ N/A

33

2.44

NORTHEAST GAS MARKETS L.L.C.

Enron Capital \& Trade Resources Canada Long Island Lighting Company

Waddington, NY

$0 \quad$ N/A

$84 \quad 2.44$

O N/A

$84 \quad 2.44$

NORTHEAST GAS MARKETS L.L.C.

Enron Capital \& Trade Resources Canada N.Y. State Electric \& Gas

Waddington, NY

$0 \quad$ N/A

$39 \quad 2.44$

$0 \quad$ N/A

$39 \quad 2.44$

NORTHEAST GAS MARKETS LL.C.

Enron Capital \& Trade Resources Canada

Southem Connecticut Gas Company

NORTHEAST GAS MARKETS L.L.C.

ProGas Limited

Central Hudson Gas \& Electric

Waddington, NY

- N/A

$5 \quad 2.28$

o N/A

$5 \quad 2.28$

NORTHEAST GAS MARKETS LL.C.

ProGas Limited

Consolidated Edison
Waddington, NY

- N/A

$21 \quad 2.28$

O N/A

$21 \quad 2.28$ 
SHORT-TERM SALES

YEAR: 1999 Quarter: Four

Estimated Volumes (MMCF) \& Prices (\$ / MMBTU)
Importer

Seller

Purchaser / End User

NORTHEAST GAS MARKETS L.L.C.

ProGas Limited

Long Island Lighting Company

NORTHEAST GAS MARKETS L.L.C.

ProGas Limited

N.Y. State Electric \& Gas

NORTHEAST GAS MARKETS L.LC.

ProGas Limited

Now Jersey Natural Gas

NORTHEAST GAS MARKETS L.L.C. ProGas Limited

Southem Connecticut Gas Company

NORTHEAST GAS MARKETS L.L.C.

ProMark

Central Hudson Gas \& Electric

NORTHEAST GAS MARKETS L.L.C.

ProMark

Consolidated Edison

NORTHEAST GAS MARKETS LLC.

ProMark

Long Island Lighting Company

Waddington, NY

Waddington, NY

- N/A

$6 \quad 2.28$

O N/A

$6 \quad 2.28$

NORTHEAST GAS MARKETS LLLC. ProMark

Now Jorsey Natural Gas

Waddington, NY

- N/A

$9 \quad 2.28$

- N/A

$9 \quad 2.28$

NORTHEAST GAS MARKETS L.L.C. ProMark

Southem Connecticut Gas Company

NORTHEAST GAS MARKETS L.LC. TransCanada Gas Services Limited Bay State Gas Company

NORTHEAST GAS MARKETS LLL.C. TransCanada Gas Services Limited Boston Gas Company
Waddington, NY

- N/A

$7 \quad 2.28$

- N/A

Niagara Falls, NY

O N/A

132

2.24

o N/A

132

2.24

Niagara Falls, NY $\begin{array}{llll}0 & \text { N/A } & 134 & 2.19\end{array}$

o N/A

134

2.19
$7 \quad 2.28$ 


\section{SHORT-TERM SALES \\ YEAR: 1999 Quarter: Four \\ Estimated Volumes (MMCF) \& Prices (\$ / MMBTU)}

Importer

Seller

Purchaser/ End User

NORTHEAST GAS MARKETS L.L.C. TransCanada Gas Services Limited

Boston Gas Company

NORTHEAST GAS MARKETS L.L.C.

TransCanada Gas Services Limited

Central Hudson Gas \& Electric

NORTHEAST GAS MARKETS L.L.C.

TransCanada Gas Services Limited

Commonwealth Energy Services

NORTHEAST GAS MARKETS LLLC.

TransCanada Gas Services Limited

Consolidated Edison

NORTHEAST GAS MARKETS LL.C.

TransCanada Gas Services Limited

Consolidated Edison

NORTHEAST GAS MARKETS LLC.

TransCanada Gas Services Limited

Energy North Inc.

NORTHEAST GAS MARKETS L.L.C. TransCanada Gas Services Limited Energy North Inc.

NORTHEAST GAS MARKETS L.L.C. TransCanada Gas Services Limited Long Island Lighting Company

Niagara Falls, NY

$0 \quad$ N/A

$28 \quad 2.20$

$0 \quad$ N/A

28

2.20

NORTHEAST GAS MARKETS L.L.C. TransCanada Gas Services Limited Long Island Lighting Company

Waddington, NY

$0 \quad$ N/A

56

2.20

$0 \quad$ N/A

561

2.20

NORTHEAST GAS MARKETS LLC.

TransCanada Gas Services Limited

N.Y. State Electric \& Gas

Waddington, NY

- N/A

121

2.21

$0 \quad$ N/A

121

2.21

NORTHEAST GAS MARKETS LL.C.

TransCanada Gas Services Limited

National Fuel Gas Distribution

Niagara Falls, NY

$0 \quad$ N/A

$28 \quad 2.18$

$0 \quad$ N/A

28

2.18

NORTHEAST GAS MARKETS LLC.

TransCanada Gas Services Limited

National Fuel Gas Distribution

Vol. ${ }^{\text {Avg. }}$ Price

184

2.19

2.19

2.19

2.20

.20

20

Waddington, NY

Quarterly 


\section{SHORT-TERM SALES}

YEAR: 1999 Quarter: Four

Estimated Volumes (MMCF) \& Prices (\$/MMBTU)

Importer

Seller

Purchaser / End User

NORTHEAST GAS MARKETS L.L.C.

TransCanada Gas Services Limited

Now Jersey Natural Gas

NORTHEAST GAS MARKETS L.L.C.

TransCanada Gas Services Limited

Public Service Gas \& Electric

NORTHEAST GAS MARKETS LL.C.

TransCanada Gas Services Limited

Southem Connecticut Gas Company

NORTHERN STATES POWER COMPANY (WI)

Husky Oil

Northem States Power (WI)

Noyes, Minnesota

NORTHERN STATES POWER COMPANY (WI)

TransCanada Gas Services Limited

Northem States Power (WI).

NORTHWEST NATURAL GAS COMPANY

Aquila Canada Corporation

Northwest Natural Gas Company

NORTHWEST NATURAL GAS COMPANY

El Paso Energy Markeing Canada

Northwest Natural Gas Company

NORTHWEST NATURAL GAS COMPANY

Engage Energy Canada

Nonthwest Natural Gas Company

NORTHWEST NATURAL GAS COMPANY

Reliant Energy Services Canada, Inc.

Northwest Natural Gas Company

NORTHWEST NATURAL GAS COMPANY

TransCanada Gas Services Limited

Northwest Natural Gas Company

Noyes, Minnesota

$0 \quad$ N/A

148

3.67

15

2.78

$301 \quad 3.22$

Sumas, Washington

$253 \quad 2.38$

o N/A

- N/A

253

2.38

Sumas, Washington

$0 \quad N / A$

444

2.20

$459 \quad 2.20$

902

2.20

Sumas, Washington

O N/A

473

2.50

488

2.42

961

2.46

Sumas, Washington

o N/A

148

2.51

153

2.47

301

2.49

Sumas, Washington

- N/A

195

2.32

$207 \quad 2.32$

401

2.32

NUMAC ENERGY (U.S.) INC.

Numac Energy

CXY Energy Marketing

Port of Morgan, MT

$161 \quad 2.20$

153

2.80

$158 \quad 1.88$

$471 \quad 2.29$

NUMAC ENERGY (U.S.) INC.

Numac Energy

Enron Capital \& Trade Resources
Port of Morgan, MT
$321 \quad 2.15$

$0 \quad$ N/A

$0 \quad$ N/A

321

2.15 


\section{SHORT-TERM SALES \\ YEAR: 1999 Quarter: Four \\ Estimated Volumes (MMCF) \& Prices (\$ / MMBTU)}

Importer

Seller
Purchaser / End User

NUMAC ENERGY (U.S.) INC.

Numac Energy

National Gas Resources

NUMAC ENERGY (U.S.) INC.

Numac Energy

Peoples Natural Gas Company

NUMAC ENERGY (U.S.) INC.

Numac Energy

Tenaska Marketing Ventures

NUMAC ENERGY (U.S.) INC.

Numac Energy.

Tenaska Washington Partners

NUMAC ENERGY (U.S.) INC.

Numac Energy

TransCanada Gas Services

PAN-ALBERTA GAS (U.S.), INC.

Pan-Alberta Gas Ltd.

Barret Resources Comoration

PAN-ALBERTA GAS (U.S.), INC.

Pan-Alberta Gas Ltd.

El Paso Energy Marketing

PAN-ALBERTA GAS (U.S.), INC.

Pan-Alberta Gas Ltd.

Enron Capital \& Trade Resources

PAN-ALBERTA GAS (U.S.), INC.

Pan-Alberta Gas Ltd.

Enserco Energy, Inc.

PAN-ALBERTA GAS (U.S.), INC.

Pan-Alberta Gas Ltd.

Fuel Imbalance

PAN-ALBERTA GAS (U.S.), INC.

Pan-Albertà Gas Ltd.

Puget Sound Energy

PAN-ALBERTA GAS (U.S.), INC.

Pan-Alberta Gas Ltd.

Puget Sound Energy
Port of Morgan, MT

- N/A

- N/A

$\begin{array}{ll}3 & 1.77\end{array}$

$\begin{array}{ll}3 & 1.77\end{array}$

Eastport, Idaho

Port of Morgan, MT

Port of Morgan, MT

Port of Morgan, MT

Eastport, Idaho

Sumas, Washington

Port of Morgan, MT

Sumas, Washington

Sumas, Washington

$9 \quad 2.72$

$13 \quad 2.12$

o N/A

22

2.37

Eastport, Idaho

Sumas, Washington
- N/A

o N/A

304

$304 \quad 1.68$

$304 \quad 1.68$

$5 \quad 2.72$

- N/A

O N/A

$5 \quad 2.72$ 


\section{SHORT-TERM SALES}

YEAR: 1999 Quarter: Four

Estimated Volumes (MMCF) \& Prices (\$ / MMBTU)

Importer

Seller

Purchaser / End User

PAN-ALBERTA GAS (U.S.), INC.

Pan-Alberta Gas Ltd.

Sempra Energy Trading Corp.

PAN-ALBERTA GAS (U.S.), INC.

Pan-Alberta Gas Ltd.

U.S. Gas Transportation Inc.

PANCANADIAN PETROLEUM COMPANY

PanCanadian Petroleum Ltd.

Various CA State Markets

PANCANADIAN PETROLEUM COMPANY

PanCanadian Petroleum Lid.

Various IL State Markets

PAWTUCKET POWER ASSOCIATES L.P.

Pawtucket Power Associates

Duke Energy Trading \& Marketing

PAWTUCKET POWER ASSOCIATES L.P.

Pawtucket Power Associates

Sempra Energy Trading Corp.

PAWTUCKET POWER ASSOCIATES L.P. Pawtucket Power Associates

Southem Company Energy

\section{PEMEX GAS}

PEMEX

MGI

\section{PEMEX GAS}

PEMEX

MGI

PETRO-CANADA HYDROCARBONS, INC. Petro-Canada Hydrocarbons Inc.

Aquila Canada Corporation

PETRO-CANADA HYDROCARBONS, INC. Petro-Canada Hydrocarbons inc.

Coral Energy Resources

PETRO-CANADA HYDROCARBONS, INC. Petro-Canada Hydrocarbons Inc.

Dynegy Marketing \& Trade
Port of Morgan, MT

- N/A

578

3.11

Entry

Sumas, Washington

Eastport, Idaho

Eastport, Idaho

Port of Morgan, MT

Waddington, NY

Waddington, NY

Waddington, NY

Alamo, Texas

Hidalgo, Texas

Eastport, Idaho

$\begin{array}{llll}\text { October } & \text { November } & \text { Quatal } \\ \text { Vol. Price } & \text { Vol. Price } & \text { Pol. Price } & \text { Avg. } \\ \text { Vol. Price }\end{array}$

o N/A

$10 \quad 2.72$

12

$122 \quad 1.68$

245

$8906 \quad 2.62$

2.42

15645

2.59

$4 \quad 2.71$

$\begin{array}{ll}4 & 2.71\end{array}$

Port of Morgan, MT

O N/A

- N/A

$42 \quad 2.99$

Port of Morgan, MT

$1077 \quad 2.23$

- N/A

- N/A

$1077 \quad 2.23$ 


\section{SHORT-TERM SALES \\ YEAR: 1999 Quarter: Four \\ Estimated Volumes (MMCF) \& Prices (\$ / MMBTU)}

Importer

Seller

Purchaser/End User

PETRO-CANADA HYDROCARBONS, INC.

Petro-Canada Hydrocarbons inc.

Engage Energy U.S. LP

PETRO-CANADA HYDROCARBONS, INC.

Petro-Canada Hydrocarbons inc.

Engage Energy U.S. LP

PETRO-CANADA HYDROCARBONS, INC.

Petro-Canada Hydrocarbons Inc.

Enron Capital \& Trade Resources

PETRO-CANADA HYDROCARBONS, INC.

Petro-Canada Hydrocartons Inc.

Famland Industries

PETRO-CANADA HYDROCARBONS, INC.

Petro-Canada Hydrocarbons Inc.

IGI Resources

Port of Morgan, MT

- N/A

- N/A

$299 \quad 2.37$

299

2.37

PETRO-CANADA HYDROCARBONS, INC.

Petro-Canada Hydrocarbons Inc.

IGI Resources

Sumas, Washington

$299 \quad 1.85$

$290 \quad 2.95$

- N/A

589

2.39

PETRO-CANADA HYDROCARBONS, INC.

Petro-Canada Hydrocarbons Inc.

Northshore Gas Company

Port of Morgan, MT

$60 \quad 2.56$

O N/A

- N/A

$60 \quad 2.56$

PETRO-CANADA HYDROCARBONS, INC

Petro-Canada Hydrocarbons inc.

Peoples Gas, Light, \& Coke

Port of Morgan, MT

$240 \quad 251$

435

3.07

$449 \quad 2.19$

1123

2.60

Port of Morgan, MT

- N/A

144

2.27

$150 \quad 2.13$

293

2.20

PETRO-CANADA HYDROCARBONS, INC.

Petro-Canada Hydrocarbons Inc.

Sierra Pacific Power Company

Sumas, Washington

- N/A

14

2.15

150

2.23

294

2.19

PETRO-CANADA HYDROCARBONS, INC. Petro-Canada Hydrocarions Ine.

Tenaska Marketing Ventures

Port of Morgan, MT

$475 \quad 2.27$

PG\&E CORE PROCUREMENT DEPT.

Amoco Canada Petroleum Company Ltd.

PG\&E Core Procurement Dept.
1255

2.17

$2954 \quad 2.58$
Total

Vol. Price

2.00

2.21

2.39

.49

37
Eastport, Idaho

$868 \quad 2.31$
978

2.72 
SHORT-TERM SALES

YEAR: 1999 Quarter: Four

Estimated Volumes (MMCF) \& Prices (\$ / MMBTU)

Importer

Seller

Purchaser / End User

PG\&E CORE PROCUREMENT DEPT.

Aquila Canada Corporation

PG\&E Core Procurement Dept.

PG\&E CORE PROCURÉMENT DEPT.

Coast Energy Canada

PG\&E Core Procurement Dept.

PG\&E CORE PROCUREMENT DEPT.

Cook Inlet Energy Supply

PG\&E Core Procurement Dept.

PG\&E CORE PROCUREMENT DEPT.

Duke Energy Marketing Canada

PG\&E Core Procurement Dept.

PG\&E CORE PROCUREMENT DEPT.

Dynegy Canada

PG\&E Core Procurement Dept.

PG\&E CORE PROCUREMENT DEPT.

Enron Capital \& Trade Resources Canada

PG\&E Core Procurement Dept.

PG\&E CORE PROCUREMENT DEPT.

ICC Energy Corporation

PG\&E Core Procurement Dept.

PG\&E CORE PROCUREMENT DEPT.

J. Aron \& Company

PG\&E Core Procurement Dept.

PG\&E CORE PROCUREMENT DEPT.

Koch Gas Services Canada

PG\&E Core Procurement Dept.

Eastport, Idaho

$145 \quad 2.34$

o N/A

$35 \quad 2.00$

180

2.27

PG\&E CORE PROCUREMENT DEPT.

Natural Gas Exchange

PG\&E Core Procurement Dept.

PG\&E CORE PROCUREMENT DEPT.

$P G \& E$ Energy Trading

$P G \& E$ Core Procurement Dept.

Eastport, ldaho

PG\&E CORE PROCUREMENT DEPT.

Pan-Alberta Gas Lid.

PG\&E Core Procurement Dept.

Point

Entry

Eastport, Idaho

$434 \quad 2.28$

$2939 \quad 2.30$

$1898 \quad 2.77$

$1986 \quad 2.33$

$6823 \quad 2.44$

Eastport, Idaho

729

434

2.31

420

2.70

$651 \quad 2.29$

1506

2.41

Eastport, Idaho

106

3.22

$340 \quad 2.08$

$2349 \quad 2.33$

N/A

84

2.68

868

2.29

1709

2.48

Eastport, Idaho
$145 \quad 2.31$
- N/A
145

2.31 


\section{SHORT-TERM SALES \\ YEAR: 1999 Quarter: Four \\ Estimated Volumes (MMCF) \& Prices (\$ / MMBTU)}

Importer

Seller

Purchaser/End User

Point

Entry

Eastport, Idaho

Eastport, Idaho

Eastport, Idaho

$46 \quad 2.72$

$0 \quad$ N/A

o N/A

46

2.72

PG\&E CORE PROCUREMENT DEPT.

Westem Gas Resources Canada

PG\&E Core Procurement Dept.

Eastport, Idaho

$579 \quad 2.31$

280

2.70

$289 \quad 2.29$

$1149 \quad 2.40$

POCO MARKETING LTD.

Poco Marketing Ltd.

Amoco Trading

Eastport, Idaho

$0 \quad$ N/A

$452 \quad 2.61$

$5 \quad 1.98$

$456 \quad 2.60$

POCO MARKETING LTD.

Poco Marketing Ltd.

Amoco Trading

POCO MARKETING LTD.

Poco Marketing Ltd.

Cargill Energy Division

POCO MARKETING LTD.

Poco Marketing Ltd.

Cibola Corporation

POCO MARKETING LTD.

Poco Marketing Ltd.

Cibola Corporation

Port of Morgan, MT

432

2.26

33

1.84

$333 \quad 1.86$

$1095 \quad 2.01$

POCO MARKETING LTD.

Poco Marketing Ltd.

Coast Energy Group

POCO MARKETING LTD.

Poco Marketing Ltd.

Commonwealth Edison

POCO MARKETING LTD.

Poco Marketing Ltd.

Commonwealth Edison
Eastport, Idaho

Noyes, Minnesota

Port of Morgan, MT
$244 \quad 2.43$

2.21

286

1.97

548

2.18

$26 \quad 2.60$

o N/A

N/A

26

2.60
$103 \quad 2.62$

- N/A

0

$0 \quad$ N/A

$103 \quad 2.62$ 


\section{SHORT-TERM SALES \\ YEAR: 1999 Quarter: Four \\ Estimated Volumes (MMCF) \& Prices (\$ / MMBTU)}

Importer

Seller

Purchaser/End User

POCO MARKETING LTD.

Poco Marketing Ltd.

Coral Redwood

POCO MARKETING LTD.

Poco Marketing Ltd.

Dynegy Marketing \& Trade

POCO MARKETING LTD.

Poco Marketing Ltd.

HL Power

Eastport, Idaho

$33 \quad 2.36$

$37 \quad 2.06$

$51 \quad 2.01$

120

2.12

POCO MARKETING LTD. Poco Marketing Ltd.

|GI Resources

POCO MARKETING LTD

Poco Marketing Ltd.

IGI Resources

Sumas, Washington

$611 \quad 2.33$

- N/A

o N/A

$611 \quad 2.33$

POCO MARKETING LTD. .

Poco Marketing Ltd.

K-N Gas Marketing

POCO MARKETING LTD.

Poco Marketing Ltd.

Nicor Gas Company

Port of Morgan, MT

$0 \quad$ N/A

$0 \quad$ N/A

$8 \quad 1.68$

$8 \quad 1.68$

Port of Morgan, MT

$794 \quad 2.16$

$51 \quad 2.03$

$0 \quad$ N/A

845

2.15

POCO MARKETING LTD.

Poco Marketing Ltd.

OGE Energy Resources

POCO MARKETING LTD.

Poco Marketing Ltd.

Pancanadian Energy Services

POCO MARKETING LTD.

Poco Marketing Ltd.

Pancanadian Energy Services

Noyes, Minnesota

$39 \quad 2.28$

$0 \quad$ N/A

- N/A

39

2.28

POCO MARKETING LTD.

Poco Marketing Ltd.

Pancanadian Energy Services

Port of Morgan, MT

$196 \quad 2.20$

185

1.84

1.88

$511 \quad 1.99$

POCO MARKETING LTD.

Poco Marketing Ltd.

Peoples Gas, Light, \& Coke
Noyes, Minnesota

$6 \quad 2.35$

o N/A

$0 \quad$ N/A

$6 \quad 2.35$ 


\section{SHORT-TERM SALES \\ YEAR: 1999 Quarter: Four \\ Estimated Volumes (MMCF) \& Prices (\$ / MMBTU)}

Importer

Seller

Purchaser / End User

POCO MARKETING LTD.

Poco Marketing Ltd.

Peoples Gas, Light, \& Coke

POCO MARKETING LTD.

Poco Marketing Ltd.

Poco Marketing

POCO MARKETING LTD.

Poco Marketing Ltd.

Puget Sound Energy

POCO MARKETING LTD.

Poco Marketing Ltd.

Quick Trade

POCO MARKETING LTD.

Poco Marketing Ltd.

Reliant Energy Services, Inc.

POCO MARKETING LTD.

Poco Marketing Ltd.

Sempra Energy Trading Corp.

Niagara Falls, NY

Port of Morgan, MT

Eastport, Idaho

Port of Morgan, MT

Port of Morgan, MT

POCO MARKETING LTD.

Poco Marketing Ltd.

Point

Entry

Port of Morgan, MT

Eastport, Idaho

Sumas, Washington

Port of Morgan, MT

Port of Morgan, MT
Tenaska Marketing Ventures

- N/A

$488 \quad 2.63$

$10 \quad 2.05$

o N/A

$795 \quad 2.41$

$3 \quad 2.02$

O N/A

31

1.68

33

1.71

PORTLAND GENERAL ELECTRIC COMPANY

Amoco Canada Petroleum Company Ltd.

Portland General Electric Company

Eastport, Idaho

$0 \quad$ N/A

282

146

4.18

427

2.76

PORTLAND GENERAL ELECTRIC COMPANY

Aquila Canada Corporation

Portiand General Electric Company
Eastport, Idaho
$437 \quad 1.70$

$141 \quad 2.00$

146

2.06

$723 \quad 1.83$ 


\section{SHORT-TERM SALES}

YEAR: 1999 Quarter: Four Estimated Volumes (MMCF) \& Prices (\$ / MMBTU)

Importer
Seller
Purchaser / End User
PORTLAND GENERAL ELECTRIC COMPANY
Coral Energy Canada Inc.
Portland General Electric Company

Eastport, Idaho

Point

Entry

PORTLAND GENERAL ELECTRIC COMPANY

Crestar Energy

Portland General Electric Company

Eastport, Idaho

$364 \quad 2.31$

o N/A

$0 \quad$ N/A

364

2.31

PORTLAND GENERAL ELECTRIC COMPANY

Duke Energy Marketing Canada

Portland General Electric Company

Eastport, Idaho

$541 \quad 1.81$

423

1.77

$437 \quad 1.81$

1401

1.80

PORTLAND GENERAL ELECTRIC COMPANY

Engage Energy Canada

Portland General Electric Company

Eastport, Idaho

O N/A

$11 \quad 1.85$

$0 \quad$ N/A

$11 \quad 1.85$

PORTLAND GENERAL ELECTRIC COMPANY J. Aron \& Company :

Portland General Electric Company

Eastport, Idaho

- N/A

$0 \quad$ N/A

$146 \quad 2.58$

$146 \quad 2.58$

PORTLAND GENERAL ELECTRIC COMPANY

Koch Gas Services Canada

Portland General Electric Company

Eastport, Idaho

$0 \quad$ N/A

$141 \quad 2.21$

$146 \quad 2.25$

287

2.23

PORTLAND GENERAL ELECTRIC COMPANY

Morgan Stanley Capital Company

Portland General Electric Company

Eastport, Idaho

- N/A

$0 \quad$ N/A

$146 \quad 2.59$

146

2.59

PORTLAND GENERAL ELECTRIC COMPANY

PG\&E Energy Trading

Portland General Electric Company

Eastport, Idaho

$247 \quad 1.63$

$141 \quad 1.92$

$146 \quad 1.96$

534

1.80

PORTLAND GENERAL ELECTRIC COMPANY

Westem Gas Resources Canada

Portland General Electric Company

Eastport, Idaho

- N/A

$141 \quad 1.92$

$146 \quad 1.96$

287

1.94

PREMSTAR ENERGY CANADA, LTD.

Premstar Energy Canada, Ltd.

CMS Gas Marketing

St. Clair, Michigan

$378 \quad 2.61$

O N/A

o N/A

$378 \quad 2.61$

PROGAS U.S.A., INC.

ProGas Limited

CXY Energy Marketing

Noyes, Minnesota

$26 \quad 2.69$

$148 \quad 3.25$

$172 \quad 2.44$

$346 \quad 2.81$

PROGAS U.S.A., INC.

ProGas Limited

Cargill Energy Division
Port of Morgan, MT

$128 \quad 2.52$
- N/A

128 


\section{SHORT-TERM SALES \\ YEAR: 1999 Quarter: Four \\ Estimated Volumes (MMCF) \& Prices (\$/MMBTU)}

Importer

Seller

Purchaser/End User

PROGAS U.S.A., INC.

ProGas Limited

Carthage Energy Services

PROGAS U.S.A., INC.

ProGas Limited

Cibola Corporation

PROGAS U.S.A., INC.

ProGas Limited

City of Two Habors, MI

PROGAS U.S.A., INC.

ProGas Limited

Delmarva Power \& Light Company

PROGAS U.S.A., INC.

ProGas Limited

Dynegy Marketing \& Trade

PROGAS U.S.A., INC.

ProGas Limited

Dynegy Marketing \& Trade

PROGAS U.S.A., INC.

ProGas Limited

Dynegy Marketing \& Trade

PROGAS U.S.A., INC.

ProGas Limited

Energy Masters Intemational, Inc.

PROGAS U.S.A., INC.

ProGas Limited

Energy Masters Intemational, Inc.

Port of Morgan, MT

Niagara Falls, NY

- N/A

227

2.95

$459 \quad 2.38$

$685 \quad 2.57$

PROGAS U.S.A., INC.

ProGas Limited

Engage Energy U.S. LP

PROGAS U.S.A., INC.

ProGas Limited

Engage Energy U.S. LP

Point
of

Entry

October

Vol. Price

November

Vol. Price

December

Vol. Price

Quarterly

Total

Vol. Price

Noyes, Minnesota

$30 \quad 2.69$

$16 \quad 3.25$

$28 \quad 2.44$

$\begin{array}{ll}75 & 2.72\end{array}$

Port of Morgan, MT

$\begin{array}{llll}0 & \text { N/A } & 181 & 2.88\end{array}$

$0 \quad$ N/A

$181 \quad 2.88$

Port of Morgan, MT

$18 \quad 2.52$

o N/A

- N/A

$18 \quad 2.52$

Niagara Falls, NY

$\begin{array}{ll}764 & 2.78\end{array}$

$2 \quad 2.95$

O N/A

766

2.78

Niagara Falls, NY

o N/A

$48 \quad 2.95$

$0 \quad$ N/A

$48 \quad 2.95$

Noyes, Minnesota

- N/A

- N/A

$153 \quad 2.44$

153

2.44

Port of Morgan, MT

- N/A

195

2.88

$50 \quad 2.44$

$246 \quad 2.79$

Niagara Falls, NY

$0 \quad$ N/A

$89 \quad 2.95$

$6 \quad 2.33$

$95 \quad 2.91$

Noyes, Minnesota
$0 \quad$ N/A

\begin{tabular}{l}
18 \\
\hline .25
\end{tabular}
- N/A
$18 \quad 3.25$ 


\section{SHORT-TERM SALES \\ YEAR: 1999 Quarter: Four \\ Estimated Volumes (MMCF) \& Prices (\$ / MMBTU)}

Importer

Seller

Purchaser/End User

PROGAS U.S.A., INC.

ProGas Limited

Honeymead Products Company

PROGAS U.S.A., INC.

ProGas Limited

Howard Energy Company

PROGAS U.S.A., INC.

ProGas Limited

Kaztex Energy Management

PROGAS U.S.A., INC.

ProGas Limited

KeySpan Gas East Corporation

PROGAS U.S.A., INC.

ProGas Limited

OGE Energy Resources

PROGAS U.S.A., INC.

ProGas Limited

PG\&E Energy Trading

PROGAS U.S.A., INC.

ProGas Limited

Pancanadian Energy Services

PROGAS U.S.A., INC.

ProGas Limited

Pancanadian Energy Services

PROGAS U.S.A., INC.

ProGas Limited

Sempra Energy Trading Corp.

PROGAS U.S.A., INC.

ProGas Limited

Southem Company Energy

PROGAS U.S.A., INC.

ProGas Limited

TXU Energy Trading Company

PROGAS U.S.A., INC.

ProGas Limited

Tenaska Marketing Ventures
Point

Entry

Port of Morgan, MT

Noyes, Minnesota

Port of Morgan, MT

Niagara Falls, NY

Port of Morgan, MT

Noyes, Minnesota

Noyes, Minnesota

Port of Morgan, MT

Noyes, Minnesota

Niagara Falls, NY

Niagara Falls, NY

$120 \quad 2.78$

$31 \quad 2.95$

22

$222 \quad 2.30$

2.30

$373 \quad 2.51$

Noyes, Minnesota

Quarterly

Total

Vol. Price

Vol. Price Vol. Price Vol. Price

$31 \quad 2.44$

$92 \quad 2.61$

$455 \quad 2.79$

$347 \quad 2.64$

$75 \quad 2.95$

$436 \quad 2.63$

$766 \quad 2.69$

$603 \quad 2.84$

2.78

$228 \quad 2.82$

$\begin{array}{llllllll}0 & \text { N/A } & 0 & \text { N/A } & 152 & 2.33 & 152 & 2.33\end{array}$

$\begin{array}{llllllll}138 & 2.69 & 0 & \text { N/A } & 0 & \text { N/A } & 138 & 2.69\end{array}$ 


\section{SHORT-TERM SALES \\ YEAR: 1999 Quarter: Four \\ Estimated Volumes (MMCF) \& Prices (\$ / MMBTU)}

Importer

Seller

Purchaser/End User

PROGAS U.S.A., INC.

ProGas Limited

Tenaska Marketing Ventures

PROGAS U.S.A., INC.

ProGas Limited

U.S. Energy Services

PROGAS U.S.A., INC.

ProGas Limited

WPS Energy Services, Inc.

PUGET SOUND ENERGY, INC.

Amoco Canada Petroleum Company Ltd.

Puget Sound Energy

PUGET SOUND ENERGY, INC.

Aquila Canada Corporation

Puget Sound Energy

PUGET SOUND ENERGY, INC.

B.C. Gas Inc.

Puget Sound Energy

PUGET SOUND ENERGY, INC.

CanWest Gas Supply Inc.

Puget Sound Energy

Sumas, Washington

Sumas, Washington

$14 \quad 2.31$

O

N/A

O N/A

14

2.31

PUGET SOUND ENERGY, INC. Duke Energy Marketing Canada Puget Sound Energy

Sumas, Washington

$44 \quad 2.36$

10

2.55

75

2.15

129

2.25

PUGET SOUND ENERGY, INC.

Encogen

Puget Sound Energy

Sumas, Washington

O N/A

$12 \quad 3.02$

- N/A

$12 \quad 3.02$

PUGET SOUND ENERGY, INC.

Engage Energy Canada

Puget Sound Energy

Sumas, Washington

$10 \quad 2.25$

$387 \quad 2.70$

o N/A

397

2.69

PUGET SOUND ENERGY, INC.

Idaho Power

Puget Sound Energy
Sumas, Washington
- N/A

29

296
$300 \quad 2.20$

$596 \quad 2.52$ 


\section{SHORT-TERM SALES \\ YEAR: 1999 Quarter: Four \\ Estimated Volumes (MMCF) \& Prices (\$ /MMBTU)}

Importer

Seller

- Rurchaser / End User

PUGET SOUND ENERGY, INC.

PG\&E Energy Trading

Puget Sound Energy

PUGET SOUND ENERGY, INC.

Powerex

Puget Sound Energy

PUGET SOUND ENERGY, INC.

Sempra Energy Trading

Puget Sound Energy

PUGET SOUND ENERGY, INC.

Talisman LT

Puget Sound Energy

QUESTAR ENERGY TRADING COMPANY

Questar Energy

Barret Resources Corporation

QUESTARENERGY TRADING COMPANY

Questar Energy

Puget Sound Energy

QUESTAR ENERGY TRADING COMPANY

Questar Energy

Statoil Energy Services, Inc.

RELIANT ENERGY SERVICES, INC.

Reliant Energy Services Canada, Inc.

Reliant Energy Services, Inc.

RELIANT ENERGY SERVICES, INC.

Reliant Energy Services Canada, Inc.

Reliant Energy Services, Inc.

RENAISSANCE ENERGY (U.S.), INC.

Renaissance Energy Ltd.

Renaissance Energy (U.S.) Inc.

RENAISSANCE ENERGY (U.S.), INC.

Renaissance Energy Ltd.

Renaissance Energy (U.S.) Inc.

RENAISSANCE ENERGY (U.S.), INC.

Renaissance Energy Ltd.

Renaissance Energy (U.S.) Inc.
Point

Of Entry

October

Vol. Price

November

Vol. Price

December

Vol. Price

Quarterly

Total

Avg.

Vol. "Price

Sumas, Washington

$\begin{array}{ll}79 & 2.31\end{array}$

$17 \quad 2.49$

O N/A

96

2.34

Sumas, Washington

118

2.29

24

2.28

$165 \quad 2.28$

524

2.28

Sumas, Washington

O N/A

$14 \quad 2.62$

- N/A

$14 \quad 2.62$

Sumas, Washington

2.54

260

2.96

263

2.32

$823 \quad 2.60$

Sumas, Washington

- N/A

92.60

- N/A

$9 \quad 2.60$

Sumas, Washington

- N/A

$27 \quad 2.67$

$9 \quad 2.01$

$37 \quad 2.50$

Sumas, Washington

- N/A

$4 \quad 2.68$

$300 \quad 2.26$

303

2.26

Eastport, Idaho

34

50

1024

2.19

$\begin{array}{ll}1358 & 2.17\end{array}$

2728

2.23

Sumas, Washington

530

2.57

446

2.09

$352 \quad 2.19$

1328

2.31

Grand Island, NY

$\begin{array}{ll}73 & 2.43\end{array}$

$82 \quad 2.97$

10

3.14

25

2.89

Niagara Falls, NY

- N/A

$51 \quad 2.66$

$2 \quad 2.42$

$53 \quad 2.65$

Noyes, Minnesota
$361 \quad 2.45$ 


\section{SHORT-TERM SALES \\ YEAR: 1999 Quarter: Four \\ Estimated Volumes (MMCF) \& Prices ( $/$ MMBTU)}

\begin{tabular}{ll} 
Importer & Point \\
Seller & of \\
Purchaser / End User & Entry \\
\hline
\end{tabular}

Renaissance Energy Ltd.

Renaissance Energy (U.S.) Inc.

RENAISSANCE ENERGY (U.S.), INC.

Renaissance Energy Ltd.

Renaissance Energy (U.S.) inc.

RENAISSANCE ENERGY (U.S.), INC.

Renaissance Energy Ltd.

Renaissance Energy (U.S.) Inc.
RENAISSANCE ENERGY (U.S.), INC.

Pittsburg, NH

Port of Morgan, MT

Waddington, NY

ROCHESTER GAS \& ELECTRIC CORPORATION

Dynegy Canada

Rochester Gas \& Electric Company

Grand Island, NY

$1121 \quad 2.94$

155

2.60

33

SAN DIEGO GAS \& ELECTRIC

B.C. Gas Inc.

San Diego Gas \& Electric Company

Eastport, Idaho

Eastport, Idaho

Eastport, Idaho

Eastport, Idaho

Sumas, Washington

St. Clair, Michigan

Various Suppliers

Various MI State Markets

SEMPRA ENERGY TRADING CORPORATION

Various Suppliers

Various CA State Markets

$0 \quad$ N/A

485

2.00

O N/A

$0 \quad$ N/A

$17 \quad 2.50$

17

2.50

SEMPRA ENERGY TRADING CORPORATION Various Suppliers

Various NY State Markets

Niagara Falls, NY

$1143 \quad 2.50$

564

2.50

763

2.50

2470

2.50

SEMPRA ENERGY TRADING CORPORATION Various Suppliers

Various NY State Markets
Waddington, NY

$\begin{array}{llllllll}7 & 2.50 & 1462 & 2.50 & 1499 & 2.50 & 2969 & 2.50\end{array}$




\section{SHORT-TERM SALES \\ YEAR: 1999 Quarter: Four \\ Estimated Volumes (MMCF) \& Prices (\$ / MMBTU)}

Importer

Seller

Purchaser / End User

Point

Entry

SEMPRA ENERGY TRADING CORPORATION

Vanious Suppliers

Various WIState Markets

SIERRA PACIFIC POWER COMPANY

B.C. Gas Inc.

Sierra Pacific Power Company

Eastport,-Idaho

Noyes, Minnesota

$190 \quad 2.00$

$267 \quad 2.64$

$273 \quad 2.09$

$362 \quad 2.18$

902

2.29

SIERRA PACIFIC POWER COMPANY

Duke Energy Marketing Canada

Siema Pacilic Power Company

Eastport, Idaho

$307 \quad 2.45$

297

SIERRA PACIFIC POWER COMPANY

Engage Energy Canada

Sierra Pacific Power Company

Eastport, Idaho

$459 \quad 1.42$

445

1.43

$460 \quad 1.42$

$1364 \quad 1.42$

SOUTHERN COMPANY ENERGY MARKETING L.P.

Southem Company Energy

ComGas

Niagara Falls, NY

$0 \quad$ N/A

o N/A

$276 \quad 2.35$

$276 \quad 2.35$

SPRAGUEENERGY CORPORATION

Boston Gas Company

Various MA State Markets

Niagara Falls, NY

$5 \quad 2.64$

$5 \quad 3.32$

$0 \quad$ N/A

$\begin{array}{ll}11 & 2.97\end{array}$

SPRAGUE ENERGY CORPORATION

Boston Gas Company

Various MA State Markets

Waddington, NY

$4 \quad 2.79$

$4 \quad 3.23$

$4 \quad 2.35$

$13 \quad 2.79$

SPRAGUE ENERGY CORPORATION

El Paso Gas Marketing Canada Inc.

Milford Power, L.P.

SPRAGUE ENERGY CORPORATION

Enron Gas Marketing Canada

Various MA State Markets

Niagara Falls, NY

$0 \quad N / A$

12.63

o N/A

$1 \quad 2.63$

SPRAGUE ENERGY CORPORATION

Enron Gas Marketing Canada

Various NJ State Markets

Niagara Falls, NY

28

2.86

- N/A

$0 \quad$ N/A

$28 \quad 2.86$

SPRAGUE ENERGY CORPORATION Enron Gas Marketing Canada

Various NY State Markets

Grand Island, NY

$4 \quad 2.88$

o N/A.

$0 \quad$ N/A

$4 \quad 2.88$

SPRAGUE ENERGY CORPORATION

Renaissance Energy Ltd.

Various NJ State Markets

Niagara Falls, NY

$30 \quad 2.87$

o N/A

$0 \quad$ N/A

$30 \quad 2.87$ 


\section{SHORT-TERM SALES}

YEAR: 1999 Quarter: Four

Estimated Volumes (MMCF) \& Prices (\$ / MMBTU)

Importer

Seller

Purchaser / End User

SPRAGUE ENERGY CORPORATION

Renaissance Energy Ltd.

Various NY State Markets

SPRAGUE ENERGY CORPORATION

Renaissance Energy Lid.

Various NY State Markets

SPRAGUE ENERGY CORPORATION

Renaissance Energy Ltd.

Various NY State Markets

SPRAGUE ENERGY CORPORATION

Renaissance Energy Ltd.

Vanous NY State Markets

SPRAGUE ENERGY CORPORATION

Sempra Energy Trading

Various MA State Markets

SPRAGUE ENERGY CORPORATION

TransCanada Gas Services Limited

Various MA State Markets

SPRAGUE ENERGY CORPORATION

TransCanada Gas Services Limited

Various NY State Markets

ST. LAWRENCE GAS COMPANY, INC.

Duke Energy Marketing Canada

Various NY State Markets

ST. LAWRENCE GAS COMPANY, INC.

Enron Capital \& Trade Resources Canada

Various NY State Markets

Massena, New York

ST. LAWRENCE GAS COMPANY, INC.

TransCanada Gas Services Limited

Various NY State Markets

Massena, New York

- N/A

O N/A

$6 \quad 2.62$

$6 \quad 2.62$

STAMPEDER ENERGY (U.S.) INC.

Gulf Canada Resources Limited

Avista Energy

Eastport, Idaho

$151 \quad 1.96$

146

2.35

$\cdot 15$

1.93

448

2.08

STAMPEDER ENERGY (U.S.) INC.

Gulf Canada Resources Limited

Coast Energy Group
Eastport, Idaho

$149 \quad 1.95$

2.35

15

$446 \quad 2.07$ 
SHORT-TERM SALES

YEAR: 1999 Quarter: Four

Estimated Volumes (MMCF) \& Prices (\$/MMBTU)
Importer

Seller

Purchaser/End User

STAMPEDER ENERGY (U.S.) INC.

Gulf Canada Resources Limited

Hesse Gas Company

STATOIL ENERGY SERVICES, INC.

Aquila Canada Corporation

Puget Sound Energy

STATOIL ENERGY SERVICES, INC.

Aquila Energy Marketing

Puget Sound Energy

STATOIL ENERGY SERVICES, INC.

Avista Energy Inc.

Barret Resources Corporation

STATOIL ENERGY SERVICES, INC. B.C. Gas Inc.

Barret Resources Corporation

STATOIL ENERGY SERVICES, INC.

B.C. Gas Inc.

K-N Gas Marketing

STATOIL ENERGY SERVICES, INC.

Barrett Resources

Aquila Energy Marketing

STATOIL ENERGY SERVICES, INC.

Coast Energy Canada

Barret Resources Corporation

STATOIL ENERGY SERVICES, INC.

Coast Energy Canada

KN Marketing L.P.

STATOIL ENERGY SERVICES, INC.

Coast Energy Canada

Southem Company Energy

Sumas, Washington

- N/A

$620 \quad 2.30$

o N/A

620

2.30

STATOIL ENERGY SERVICES, INC.

Cook Inlet Energy Supply

Southem Company Energy

Sumas, Washington

- N/A

o N/A

Vol. Price

November

December

Vol. Price

Quarterly

Total

Vol. Price

Sumas, Washington

1552.01

$155 \quad 2.01$

Sumas, Washington

o N/A

455

2.10

Sumas, Washington

o N/A

$45 \quad 1.86$

Sumas, Washington

Sumas, Washington

Sumas, Washington

$0 \quad$ N/A

10

2.73

STATOIL ENERGY SERVICES, INC.

Cook Inlet Energy Supply

Statoil Energy Services, Inc.
Sumas, Washington

$155 \quad 2.10$

o N/A

$155 \quad 2.10$ 


\section{SHORT-TERM SALES}

YEAR: 1999 Quarter: Four

Estimated Volumes (MMCF) \& Prices (\$/MMBTU)

Importer

Seller

Purchaser/End User

STATOIL ENERGY SERVICES, INC.

Coral Energy Canada Inc.

Enron Capital \& Trade Resources

STATOIL ENERGY SERVICES, INC.

Coral Energy Canada Inc.

Statoil Energy Services, Inc.

STATOIL ENERGY SERVICES, INC.

Duke Energy Marketing Canada

Coral Energy Resources

STATOIL ENERGY SERVICES, INC.

Duke Energy Marketing Canada

Statoil Energy Services, Inc.

STATOIL ENERGY SERVICES, INC.

Enron Capital \& Trade Resources Canada

Statoil Energy Services, Inc.

STATOIL ENERGY SERVICES, INC.

Questar Energy

Statoil Energy Services, Inc.

STATOIL ENERGY SERVICES, INC.

Reliant Energy Services Canada, Inc.

Statoil Energy Services, Inc.

STATOIL ENERGY SERVICES, INC.

TransCanada Gas Services Limited

Statoil Energy Services, Inc.

Sumas, Washington

O N/A

25

1.97

$20 \quad 2.31$

$45 \quad 2.12$

SUNCOR ENERGY INC.

Suncor Energy Inc.

Aquila Energy Marketing

Eastport, Idaho

$\begin{array}{ll}78 & 2.27\end{array}$

$75 \quad 2.55$

$\begin{array}{ll}78 & 2.00\end{array}$

$230 \quad 2.27$

SUNCOR ENERGY INC.

Suncor Energy Inc.

Engage Energy U.S. LP

Eastport, Idaho

155

2.27

150

2.55

$155 \quad 2.00$

460

2.27

SUNCOR ENERGY INC.

Suncor Energy Inc.

Pancanadian Energy Services

Eastport, Idaho

SUNCOR ENERGY INC.

Suncor Energy Inc.

Pancanadian Energy Services

Quarteriy

Total

Vol. ${ }^{\text {Avg. Price }}$

$620 \quad 2.32$

$155 \quad 2.10$

$310 \quad 2.38$

$930 \quad 2.11$

$775 \quad 2.25$

$225 \quad 2.30$

$125 \quad 2.16$

$\begin{array}{llllllll}76 & 2.27 & 0 & \text { N/A } & 0 & \text { N/A } & 76 & 2.27\end{array}$

$\begin{array}{lllllllll}\text { Port of Morgan, MT } & 124 & 2.20 & 128 & 2.70 & 131 & 1.85 & 382 & 2.25\end{array}$ 


\section{SHORT-TERM SALES}

YEAR: 1999 Quarter: Four Estimated Volumes (MMCF) \& Prices (\$ / MMBTU)
Importer

Seller

Purchaser/End User

SUNCOR ENERGY INC.

Suncor Energy Inc.

Reliant Energy Services, Inc.

SUNCOR ENERGY INC

Suncor Energy Inc.

TransCanada Energy Marketing USA

SUNCOR ENERGY INC.

Suncor Energy Inc.

Wisconsin Power \& Light

SUNOMA ENERGY CORPORATION

Sunoma Energy Corporation

El Paso Energy Marketing

TENASKA MARKETING VENTURES Talisman Energy, Inc.

Various IA State Markets

TENASKA MARKETING VENTURES Tenaska Marketing Canada

Various IA State Markets

TENASKA MARKETING VENTURES Tenaska Marketing Canada

Various MN State Markets

TENASKA MARKETING VENTURES Tenaska Marketing Ventures

Various IA State Markets

TEXACO NATURAL GAS INC.

Lockport Energy Associates, L.P.

Texaco Natural Gas

TEXACO NATURAL GAS INC.

Various Suppliers

Texaco Natural Gas

THE MEAD CORPORATION

Coral Energy Canada Inc.

Mead Paper

THE MONTANA POWER COMPANY

Dynegy Canada

Montana Power
Point

Entry

Eastport, Idaho

$469 \quad 2.27$

$555 \quad 2.55$

$399 \quad 2.00$

1422

2.30

Eastport, Idaho

$462 \quad 2.27$

$431 \quad 2.55$

$620 \quad 2.00$

1513

2.24

Port of Morgan, MT

$345 \quad 2.20$

$335 \quad 2.70$

$347 \quad 1.85$

1026

2.24

Port of Morgan, MT

- N/A

$123 \quad 3.25$

$127 \quad 2.43$

250

Port of Morgan, MT

$61 \quad 2.47$

$\begin{array}{ll}59 & 2.97\end{array}$

$61 \quad 2.09$

180

Port of Morgan, MT

$0 \quad$ N/A

$695 \quad 2.70$

25262.00

3221

Noyes, Minnesota

- N/A

$840 \quad 2.13$

- N/A

840

2.13

Port of Morgan, MT

$971 \quad 2.59$

o N/A

o N/A

971

2.59

Niagara Falls, NY

- N/A

$99 \quad 3.62$

o N/A

99

3.62

Niagara Falls, NY

$330 \quad 2.87$

o N/A

265

2.43

595

2.67

Pittsburg, NH

150

$155 \quad 3.73$

$150 \quad 2.76$

455

3.08

Whitlash, Montana
$0 \quad$ N/A

$304 \quad 2.32$
$314 \quad 2.32$

$619 \quad 2.32$ 
SHORT-TERM SALES

YEAR: 1999 Quarter: Four

Estimated Volumes (MMCF) \& Prices (\$/MMBTU)

Importer

Seller

Purchaser / End User

Point

Entry

THE MONTANA POWER TRADING \& MARKETING COMPANY

The Montana Power Trading \& Marketing Co.

Various CA State Markets

Eastport, Idaho

THE MONTANA POWER TRADING \& MARKETING COMPANY

The Montana Power Trading \& Marketing Co.

Various IL State Markets

Port of Morgan, MT

THE MONTANA POWER TRADING \& MARKETING COMPANY

The Montana Power Trading \& Marketing Co.

Various MT State Markets

Babb, Montana

THE MONTANA POWER TRADING \& MARKETING COMPANY

The Montana Power Trading \& Marketing Co.

Various MT State Markets

Port of Del Bonita,

THE MONTANA POWER TRADING \& MARKETING COMPANY

The Montana Power Trading \& Marketing Co.

Various MT State Markets

Whitlash, Montana

Waddington, NY

$3 \quad 2.67$

O N/A

$0 \quad$ N/A

$3 \quad 2.67$

TRANSCANADA GAS SERVICES INC.

TransCanada Gas Services Limited

AEP Energy Services

Eastport, Idaho

155

1.99

29

2.50

$0 \quad 2.26$

184

2.07

TRANSCANADA GAS SERVICES INC.

TransCanada Gas Services Limited

AEP Energy Services

Noyes, Minnesota

- N/A

$19 \quad 2.88$

- N/A

$19 \quad 2.88$

TRANSCANADA GAS SERVICES INC. TransCanada Gas Services Limited

AEP Energy Services

Sumas, Washington

- N/A

$16 \quad 2.26$

- N/A

$16 \quad 2.26$

TRANSCANADA GAS SERVICES INC. TransCanada Gas Services Limited Adams Resources Marketing, Ltd.

Waddington, NY

- N/A

$2 \quad 2.16$

O N/A

$2 \quad 2.16$

TRANSCANADA GAS SERVICES INC. TransCanada Gas Services Limited Alliance Energy Services

Niagara Falis, NY

$5 \quad 2.66$

o N/A

$0 \quad$ N/A

$5 \quad 2.66$

TRANSCANADA GAS SERVICES INC. TransCanada Gas Services Limited Amoco Canada Marketing Co. 
Importer

Seller

Purchaser/End User

TRANSCANADA GAS SERVICES INC.

TransCanada Gas Services Limited

Aquila Energy Marketing

TRANSCANADA GAS SERVICES INC.

TransCanada Gas Services Limited

Aquila Energy Marketing

TRANSCANADA GAS SEAVICES INC.

TransCanada Gas Services Limited

Aquila Energy Marketing

TRANSCANADA GAS SERVICES INC.

TransCanada Gas Services Limited

Avista Energy

TRANSCANADA GAS SERVICES INC.

TransCanada Gas Services Limited

Barret Resources Corporation

TRANSCANADA GAS SERVICES INC.

TransCanada Gas Services Limited

Barret Resources Corporation

$\begin{array}{llll}10 & 2.39 & 167 & 2.26\end{array}$

Eastport, Idaho

$46 \quad 1.99$

o N/A

$0 \quad$ N/A

$46 \quad 1.99$

TRANSCANADA GAS SERVICES INC.

TransCanada Gas Services Limited

CMS Gas Marketing

Noyes, Minnesota

$\begin{array}{llll}29 & 2.47 & 1329 & 2.88\end{array}$

$5 \quad 2.14$

1362

2.87

TRANSCANADA GAS SERVICES INC.

TransCanada Gas Services Limited

CXY Energy Marketing

Noyes, Minnesota

$196 \quad 2.47$

O N/A

$0 \quad$ N/A

196

2.47

TRANSCANADA GAS SERVICES INC.

TransCanada Gas Services Limited

Cabot Oil

Noyes, Minnesota

$2 \quad 2.47$

2.88

$1 \quad 2.14$

$4 \quad 2.39$

TRANSCANADA GAS SERVICES INC.

TransCanada Gas Services Limited

CalPine Fuels Corporation

Eastport, Idaho

$1 \quad 1.99$

$1 \quad 2.50$

o N/A

$2 \quad 2.19$

TRANSCANADA GAS SERVICES INC.

TransCanada Gas Services Limited

Carthage Energy Services
Noyes, Minnesota

$194 \quad 2.47$

$0 \quad$ N/A

o N/A

194

2.47 


\section{SHORT-TERM SALES \\ YEAR: 1999 Quarter: Four \\ Estimated Volumes (MMCF) \& Prices (\$/MMBTU)}

Importer

Seller

Purchaser / End User

TRANSCANADA GAS SERVICES INC.

TransCanada Gas Services limited

Central Hudson Gas \& Electric

TAANSCANADA GAS SERVICES INC.

TransCanada Gas Services Limited

Cibola Corporation

TRANSCANADA GAS SERVICES INC.

TransCanada Gas Services Limited

Cibola Corporation

TRANSCANADA GAS SERVICES INC.

TransCanada Gas Services Limited

Clinton Gas Marketing

TRANSCANADA GAS SERVICES INC.

TransCanada Gas Services Limited

CoEnergy Trading Company

Niagara Falls, NY

$21 \quad 2.66$

O N/A

- N/A

21

2.66

TRANSCANADA GAS SERVICES INC. TransCanada Gas Services Limited CoEnergy Trading Company

Noyes, Minnesota

$152 \quad 2.47$

$37 \quad 2.88$

$54 \quad 2.14$

243

2.46

TRANSCANADA GAS SERVICES INC. TransCanada Gas Services Limited Coast Energy Group

THANSCANADA GAS SERVICES INC. TransCanada Gas Services Limited Columbia Energy Services

TRANSCANADA GAS SERVICES INC. TransCanada Gas Services Limited Connecticut Natural Gas

Waddington, NY

$3 \quad 2.67$

$179 \quad 2.16$

$0 \quad$ N/A

182

TRANSCANADA GAS SERVICES INC. TransCanada Gas Services Limited

Conoco Inc.

Eastport, Idaho

11.99

$0 \quad$ N/A

O N/A

1

1.99

TRANSCANADA GAS SERVICES INC. TransCanada Gas Services Limited Consumers Energy Company

Noyes, Minnesota

$0 \quad$ N/A

$186 \quad 2.88$

$372 \quad 2.14$

558

2.39

TRANSCANADA GAS SERVICES INC. TransCanada Gas Services Limited Cook Inlet
Eastport, Idaho
$39 \quad 2.50$

12.26

$\begin{array}{ll}71 & 2.27\end{array}$ 


\section{SHORT-TERM SALES \\ YEAR: 1999 Quarter: Four}

Estimated Volumes (MMCF) \& Prices (\$ / MMBTU)

Importer

Seller

Purchaser / End User

TRANSCANADA GAS SERVICES INC.

TransCanada Gas Services Limited

Coral Energy Resources

TRANSCANADA GAS SERVICES INC.

TransCanada Gas Services Limited

Coral Energy Resources

TRANSCANADA GAS SERVICES INC

TransCanada Gas Services Limited

Coral Energy Resources

TRANSCANADA GAS SERVICES INC.

TransCanada Gas Services Limited

Detroit Edison Company

TRANSCANADA GAS SERVICES INC.

TransCanada Gas Services Limited

Duke Energy Trading \& Marketing

TRANSCANADA GAS SERVICES INC.

TransCanada Gas Services Limited

Duke Energy Trading \& Marketing

TRANSCANADA GAS SERVICES INC.

TransCanada Gas Services Limited

Dynegy Marketing \& Trade

TRANSCANADA GAS SERVICES INC.

TransCanada Gas Services Limited

Dynegy Marketing \& Trade

TRANSCANADA GAS SERVICES INC. TransCanada Gas Services Limited El Paso Energy Marketing

TRANSCANADA GAS SERVICES INC.

TransCanada Gas Services Limited

Engage Energy U.S. LP

TRANSCANADA GAS SERVICES INC.

TransCanada Gas Services Limited

Engage Energy U.S. LP

TRANSCANADA GAS SERVICES INC.

TransCanada Gas Services Limited

Enron Capital \& Trade Resources
Noyes, Minnesota

$293 \quad 2.47$

$6 \quad 2.88$

36

2.14

$335 \quad 2.44$

Point

Entry

Eastport, Idaho

$92 \quad 1.99$

$21 \quad 2.50$

$86 \quad 2.26$

199

2.16

Noyes, Minnesota

$8 \quad 2.47$

$0 \quad$ N/A

- N/A

$8 \quad 2.47$

Sumas, Washington

$\begin{array}{ll}26 & 2.39\end{array}$

$0 \quad$ N/A

$30 \quad 2.31$

$57 \quad 2.35$

Noyes, Minnesota

Eastport, Idaho

Noyes, Minnesota

Eastport, Idaho

11.99

$39 \quad 2.50$

$82 \quad 2.26$

123

2.33

Waddington, NY

- N/A

$2 \quad 2.16$

o N/A

$2 \quad 2.16$

Eastport, Idaho

$8 \quad 1.99$

$21 \quad 2.50$

$44 \quad 2.26$

$\begin{array}{ll}73 & 2.30\end{array}$

Noyes, Minnesota 364

2.47

28

Eastport, Idaho
$6 \quad 1.99$

$112 \quad 2.50$

$51 \quad 2.26$

$169 \quad 2.41$ 
SHORT-TERM SALES

YEAR: 1999 Quarter: Four Estimated Volumes (MMCF) \& Prices ( $\$$ /MMBTU)
Importer

Seller

Purchaser / End User

TRANSCANADA GAS SERVICES INC.

TransCanada Gas Services Limited

Enron Capital \& Trade Resources

TRANSCANADA GAS SERVICES INC.

TransCanada Gas Services Limited

Enron Capital \& Trade Resources

TRANSCANADA GAS SERVICES INC.

TransCanada Gas Services Limited

Enron Capital \& Trade Resources

TRANSCANADA GAS SERVICES INC.

TransCanada Gas Services Limited

Enron North America Corp.

Eastport, Idaho

$10 \quad 1.99$

TRANSCANADA GAS SERVICES INC.

TransCanada Gas Services Limited

Enserco Energy, Inc.

TRANSCANADA GAS SERVICES INC.

TransCanada Gas Services Limited

Fuel Imbalance

TRANSCANADA GAS SERVICES INC.

TransCanada Gas Services Limited

Fuel Imbalance

TRANSCANADA GAS SERVICES INC.

TransCanada Gas Services Limited

Fuel Imbalance

TRANSCANADA GAS SERVICES INC.

TransCanada Gas Services Limited

Fuel Use

Eastport, Idaho

TRANSCANADA GAS SERVICES INC.

TransCanada Gas Services Limited

IDA Corporation Energy Solutions L.P.

TRANSCANADA GAS SERVICES INC.

TransCanada Gas Services Limited

IDA Corporation Energy Solutions L.P.

Sumas, Washington

TRANSCANADA GAS SERVICES INC.

TransCanada Gas Services Limited

IGI Resources o N/A

- N/A

$10 \quad 1.99$

O N/A

- N/A

$21 \quad 2.26$

$21 \quad 2.26$

$0 \quad$ N/A

$\begin{array}{ll}7 & 2.26\end{array}$

o N/A

$7 \quad 2.26$

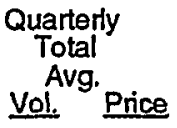

1139

2.47

\begin{tabular}{ll}
9.38 \\
\hline
\end{tabular}

$\begin{array}{ll}7 & 2.67\end{array}$

$42 \quad 2.21$

$889 \quad 2.51$

$11 \quad 2.50$

$2 \quad 2.26$

$1 \quad 2.50$

$1 \quad 2.26$

$7 \quad 1.99$

$10 \quad 2.50$

- N/A

17. 2.29
11.99
Eastport, Idaho 
SHORT-TERM SALES

YEAR: 1999 Quarter: Four Estimated Volumes (MMCF) \& Prices (\$ / MMBTU)
Importer

Seller

Purchaser / End User

Point

Entry

Port of Morgan, MT

Noyes, Minnesota

$97 \quad 2.47$

o N/A

$0 \quad$ N/A

$97 \quad 2.47$

TRANSCANADA GAS SERVICES INC. TransCanada Gas Services Limited KN Marketing L.P.

TRANSCANADA GAS SERVICES INC. TransCanada Gas Services Limited Kaztex Energy Management

TRANSCANADA GAS SERVICES INC. TransCanada Gas Services Limited KoySpan Gas East Corporation

TRANSCANADA GAS SERVICES INC. TransCanada Gas Services Limited Kimball Energy Corporation

Sumas, Washington

Babb, Montana

Noyes, Minnesota

O N/A

$2 \quad 2.88$

O N/A

$2 \quad 2.88$

TRANSCANADA GAS SERVICES INC. TransCanada Gas Services Limited

Michigan Consolidated Gas Company

TRANSCANADA GAS SERVICES INC. TransCanada Gas Services Limited Mieco

Eastport, Idaho

$46 \quad 1.99$

o N/A

O N/A

46

1.99

TRANSCANADA GAS SERVICES INC. TransCanada Gas Services Limited Montana Power

Eastport, Idaho

$0 \quad$ N/A

$21 \quad 2.50$

$43 \quad 2.26$

64

2.34

TRANSCANADA GAS SERVICES INC. TransCanada Gas Services Limited

National Fuel Gas Distribution

Quarterly
Total
Avg.

Vol. Price

132

$51 \quad 2.39$

$373 \quad 2.42$

2.55

$1 \quad 2.39$

$6 \quad 1.97$

2.65
Niagara Falls, NY
$0 \quad$ N/A
$242 \quad 2.66$ 
SHORT-TERM SALES

YEAR: 1999 Quarter: Four

Estimated Volumes (MMCF) \& Prices (\$ / MMBTU)
Importer

Seller

Purchaser/End User

TRANSCANADA GAS SERVICES INC.

TransCanada Gas Services Limited

National Fuel Resources

TRANSCANADA GAS SERVICES INC.

TransCanada Gas Services Limited

Nevada Power Company

TRANSCANADA GAS SERVICES INC.

TransCanada Gas Services Limited

New Jersey Natural Gas

TRANSCANADA GAS SERVICES INC.

TransCanada Gas Services Limited

Northeast Gas Markets

TRANSCANADA GAS SERVICES INC.

TransCanada Gas Services Limited

Northeast Gas Markets

TRANSCANADA GAS SERVICES INC.

TransCanada Gas Services Limited

Northem States Power Company

TRANSCANADA GAS SERVICES INC.

TransCanada Gas Services Limited

Northshore Gas Company

TRANSCANADA GAS SERVICES INC.

TransCanada Gas Services Limited

OGE Energy Resources

Noyes, Minnesota

O N/A

$12 \quad 2.88$

$18 \quad 2.14$

31

2.44

TRANSCANADA GAS SERVICES INC.

TransCanada Gas Services Limited

Occidental

Eastport, Idaho

$70 \quad 1.99$

$2 \quad 2.50$

- N/A

$72 \quad 2.00$

TRANSCANADA GAS SERVICES INC.

TransCanada Gas Services Limited

PG\&E Energy Trading

Eastport, Idaho

O N/A

$407 \quad 2.50$

460

2.26

867

2.37

TRANSCANADA GAS SERVICES INC.

TransCanada Gas Services Limited

PG\&E Energy Trading

Waddington, NY

- N/A

$2 \quad 2.16$

o N/A

$2 \quad 2.16$

TRANSCANADA GAS SERVICES INC.

TransCanada Gas Services Limited

Pacific Gas \& Electric Company
Eastport, ldaho

$15 \quad 2.50$

$5 \quad 2.26$

$21 \quad 2.42$ 
SHORT-TERM SALES

YEAR: 1999 Quarter: Four Estimated Volumes (MMCF) \& Prices ( $\$$ /MMBTU)
Importer

Seller

Purchaser / End User

TRANSCANADA GAS SERVICES INC.

TransCanada Gas Services Limited

Pancanadian Energy Services

TRANSCANADA GAS SERVICES INC.

TransCanada Gas Services Limited

Pancanadian Energy Services

TRANSCANADA GAS SERVICES INC.

TransCanada Gas Services Limited

Peoples Gas, Light, \& Coke

TRANSCANADA GAS SERVICES INC.

TransCanada Gas Services Limited

Poco Marketing

TRANSCANADA GAS SERVICES INC.

TransCanada Gas Services Limited

Portland General Electric Company

TRANSCANADA GAS SERVICES INC.

TransCanada Gas Services Limited

Puget Sound Energy

TRANSCANADA GAS SERVICES INC.

TransCanada Gas Services Limited

Questar Energy Trading Co.

TRANSCANADA GAS SERVICES INC.

TransCanada Gas Services Limited

Quick Trade

TRANSCANADA GAS SERVICES INC. TransCanada Gas Services Limited

Reliant Energy Services, Inc.

Noyes, Minnesota

$129 \quad 2.47$

$31 \quad 2.88$

$273 \quad 2.14$

$434 \quad 2.29$

Noyes, Minnesota

$972 \quad 2.47$

941

2.88

101

2.14

2927

2.49

Eastport, ldaho

0 - N/A

$3 \quad 2.50$

$0 \quad$ N/A

$3 \quad 2.50$

Sumas, Washington

$319 \quad 2.39$

$30 \quad 2.26$

$0 \quad$ N/A

349

2.38

Sumas, Washington

- N/A

o N/A

$67 \quad 2.31$

$67 \quad 2.31$

Sumas, Washington

$3 \quad 2.39$

O N/A

$0 \quad$ N/A

$3 \quad 2.39$

Eastport, ldaho

$0 \quad$ N/A

$22 \quad 2.50$

O N/A

22

2.50

Eastport, Idaho

$96 \quad 1.99$

$50 \quad 2.50$

$28 \quad 2.26$

174

2.18

TRANSCANADA GAS SERVICES INC.

TransCanada Gas Services Limited

Reliant Energy Services, Inc.

Noyes, Minnesota

$896 \quad 3.06$

92

2.44

$3194 \quad 2.66$

TAANSCANADA GAS SERVICES INC. TransCanada Gas Services Limited Rhone-Poulenc

Babb, Montana

$0 \quad$ N/A

$12 \quad 2.05$

O N/A

$12 \quad 2.05$

TRANSCANADA GAS SERVICES INC.

TransCanada Gas Services Limited

SCANA Energy Marketing
Eastport, Idaho $541.99^{\prime} \quad 0 \quad$ N/A
$54 \quad 1.99$ 


\section{SHORT-TERM SALES \\ YEAR: 1999 Quarter: Four \\ Estimated Volumes (MMCF) \& Prices (\$ / MMBTU)}

Importer

Seller

Purchaser/End User

TRANSCANADA GAS SERVICES INC.

TransCanada Gas Services Limited

SCANA Energy Marketing

TRANSCANADA GAS SERVICES INC.

TransCanada Gas Services Limited

Sacramento Municipal Utility Dist.

TRANSCANADA GAS SERVICES INC.

TransCanada Gas Services Limited

San Diego Gas \& Electric Company

TRANSCANADA GAS SERVICES INC.

TransCanada Gas Services Limited

Sempra Energy Trading Corp.

TRANSCANADA GAS SERVICES INC.

TransCanada Gas Services Limited

Sempra Energy Trading Corp.

TRANSCANADA GAS SERVICES INC.

TransCanada Gas Services Limited

Sierra Pacific Power Company

TRANSCANADA GAS SERVICES INC.

TransCanada Gas Services Limited

Southem Califomia Gas Company

TRANSCANADA GAS SERVICES INC.

TransCanada Gas Services Limited

Southem Company Energy

TRANSCANADA GAS SERVICES INC.

TransCanada Gas Services Limited

Southem Indiana

Noyes, Minnesota

- N/A

$8 \quad 2.88$

$0 \quad$ N/A

8

2.88

TRANSCANADA GAS SERVICES INC.

TransCanada Gas Services Limited

Southwest Gas Corporation

Sumas, Washington

$1 \quad 2.39$

$6 \quad 2.26$

$0 \quad$ N/A

$7 \quad 2.28$

TRANSCANADA GAS SERVICES INC.

TransCanada Gas Services Limited

Star Natural Gas Company

Noyes, Minnesota

$0 \quad$ N/A

31

2.88

93

2.39

TRANSCANADA GAS SERVICES INC.

TransCanada Gas Services Limited

Statoil Energy Services, Inc.
Eastport, Idaho
$0 \quad$ N/A

$1 \quad 2.50$

$0 \quad$ N/A

12.50 


\section{SHORT-TERM SALES \\ YEAR: 1999 Quarter: Four \\ Estimated Volumes (MMCF) \& Prices (\$ /MMBTU)}

Importer

Seller

Purchaser / End User

Point

Entry

Eastport, Idaho

Noyes, Minnesota

103

2.47

$4 \quad 2.88$

$4 \quad 2.14$

111

2.47

TRANSCANADA GAS SERVICES INC.

TransCanada Gas Services Limited

Tenaska Marketing Ventures

Noyes, Minnesota

$0 \quad$ N/A

$\begin{array}{ll}68 & 2.88\end{array}$

$0 \quad$ N/A

$68 \quad 2.88$

TRANSCANADA GAS SERVICES INC.

TransCanada Gas Services Limited

Texaco Natural Gas

Eastport, Idaho

$18 \quad 1.99$

$47 \quad 2.50$

o N/A

$64 \quad 2.36$

TRANSCANADA GAS SERVICES INC.

TransCanada Gas Services Limited

Texaco Natural Gas

TRANSCANADA GAS SERVICES INC.

TransCanada Gas Services Limited

Texex Energy Partners

TRANSCANADA GAS SERVICES INC.

TransCanada Gas Services Limited

TransCanada Gas Services

TRANSCANADA GAS SERVICES INC.

TransCanada Gas Services Limited

TransCanada Power (Castleton) LLC

TRANSCANADA GAS SERVICES INC.

TransCanada Gas Services Limited

U.S. Gas Transportation Inc.

Eastport, Idaho

11.99

$10 \quad 2.50$

$0 \quad$ N/A

$11 \quad 2.44$

TRANSCANADA GAS SERVICES INC.

TransCanada Gas Services Limited

U.S. Generating Company

Waddington, NY

$3 \quad 2.67$

$29 \quad 2.16$

$0 \quad$ N/A

$32 \quad 2.21$

TRANSCANADA GAS SERVICES INC. TransCanada Gas Services Limited

Utilicorp United

Noyes, Minnesota

$0 \quad$ N/A

$0 \quad$ N/A

$1 \quad 2.14$

$1 \quad 2.14$

TRANSCANADA GAS SERVICES INC. TransCanada Gas Services Limited

Virginia Power

Avg.

Vol. Price

$34 \quad 2.13$

$18 \quad 2.61$

$49 \quad 2.59$

2.27

$73 \quad 2.50$
Noyes, Minnesota

$0 \quad$ N/A

$6 \quad 2.88$

$0 \quad$ N/A

$.6 \quad 2.88$

Quarteriy 
Importer

Seller

Purchaser / End User

TRANSCANADA GAS SERVICES INC.

TransCanada Gas Services Limited

Western Gas Resources

TRANSCANADA GAS SERVICES INC.

TransCanada Gas Services Limited

Westem Gas Resources

TRANSCANADA GAS SERVICES INC.

TransCanada Gas Services Limited

Wild Goose Storage

TRANSCANADA GAS SERVICES INC.

TransCanada Gas Services Limited

Williams Energy Company

TRANSCANADA GAS SERVICES INC.

TransCanada Gas Services Limited

Williams Energy Company

TRANSCANADA GAS SERVICES INC.

TransCanada Gas Services Limited

Wisconsin Electric

TRANSCANADA GAS SERVICES INC.

TransCanada Gas Services Limited

Wisconsin Fuel \& Light

TRANSCANADA GAS SERVICES INC.

TransCanada Gas Services Limited

Wisconsin Gas

Noyes, Minnesota

$997 \quad 2.98$

$32 \quad 2.14$

$1061 \quad 2.94$

TRANSCANADA GAS SERVICES INC.

TransCanada Gas Services Limited

Wisconsin Power \& Light

Noyes, Minnesota

$2 \quad 2.47$

$43 \quad 3.06$

$6 \quad 2.14$

$50 \quad 2.94$

TRANSCANADA GAS SERVICES INC. (SP)

TransCanada Gas Services Limited

Yankee Gas Services

Niagara Falls, NY

$284 \quad 3.25$

$0 \quad N / A$

$577 \quad 2.90$

TRANSCO ENERGY MARKETING COMPANY

Aquila Canada Corporation

Various NY State Markets

Niagara Falls, NY

327

2.65

$317 \quad 3.42$

327

2.45

971

2.83

TRANSCO ENERGY MARKETING COMPANY

CXY Marketing

Various NY State Markets

Niagara Falls; NY
O N/A

$\begin{array}{ll}38 & 2.57\end{array}$
$160 \quad 2.36$

198

2.40 


\section{SHORT-TERM SALES}

YEAR: 1999 Quarter: Four

Estimated Volumes (MMCF) \& Prices (\$ / MMBTU)

Importer

Seller

Purchaser/End User

TRANSCO ENERGY MARKETING COMPANY

Coral Energy Canada Inc.

Various NY State Markets

TRANSCO ENERGY MARKETING COMPANY

Duke Energy Marketing Canada

Various NY State Markets

Niagara Falls, NY

$327 \quad 2.65$

$950 \quad 3.46$

$981 \quad 2.49$

2258

2.92

TRANSCO ENERGY MARKETING COMPANY

Dynegy Canada

Various NY State Markets

Niagara Falls, NY

$0 \quad$ N/A

- N/A

$164 \quad 2.30$

164

2.30

TRANSCO ENERGY MARKETING COMPANY Engage Energy Canada

Vanous NY State Markets

Niagara Falls, NY

654

2.68

$812 \quad 3.39$

$0 \quad$ N/A

$1467 \quad 3.07$

TRANSCO ENERGY MARKETING COMPANY

Enron Capital \& Trade Resources Canada

Various NY State Markets

Niagara Falls, NY

TAANSCO ENERGY MARKETING COMPANY Equitable Resources Marketing Canada

Various NY State Markets

Niagara Falls, NY

- N/A

o N/A

$164 \quad 2.29$

$164 \quad 2.29$

TRANSCO ENERGY MARKETING COMPANY Indeck-Yerkes Energy Services

Various NY State Markets

Niagara Falls, NY

$0 \quad$ N/A

O N/A

$61 \quad 2.41$

$61 \quad 2.41$

TRANSCO ENERGY MARKETING COMPANY

ProGas Limited

Various NY State Markets

Niagara Falls, NY

$0 \quad$ N/A

$37 \quad 2.43$

21

2.39

$59 \quad 2.42$

TRANSCO ENERGY MARKETING COMPANY

Renaissance Energy Ltd.

Various NY State Markets

Niagara Falls, NY

1123

2.64

670

3.41

789

2.58

$2583 \quad 2.82$

TRANSCO ENERGY MARKETING COMPANY

Sempra Energy Trading

Various NY State Markets

Niagara Falls, NY

- N/A

$75 \quad 2.43$

$0 \quad$ N/A

$75 \quad 2.43$

TRANSCO ENERGY MARKETING COMPANY

TXU

Various NY State Markets

Niagara Falls, NY

TRANSCO ENERGY MARKETING COMPANY

TransCanada Gas Services Limited

Various NY State Markets
O N/A

$0 \quad$ N/A

2.39

$11 \quad 2.39$

$0 \quad$ N/A

$685 \quad 2.26$

$69 \quad 2.50$

$754 \quad 2.28$ 


\section{SHORT-TERM SALES \\ YEAR: 1999 Quarter: Four \\ Estimated Volumes (MMCF) \& Prices (\$ / MMBTU)}

Importer

Seller.

Purchaser / End User

TXU ENERGY TRADING COMPANY

Avista Energy Inc.

Various CA State Markets

TXU ENERGY TRADING COMPANY

Boston Gas Company

Various NY State Markets

TXU ENERGY TRADING COMPANY

Boston Gas Company

Various NY State Markets

TXU ENERGY.TRADING COMPANY

New Jersey Natural Gas

Vanious NY State Markets

TXU ENERGY TRADING COMPANY

TXU

Various NY State Markets

UNITED STATES GYPSUM COMPANY

Coral Energy Canada Inc.

United States Gypsum

UNITED STATES GYPSUM COMPANY

Engage Energy Canada

United States Gypsum

UNITED STATES GYPSUM COMPANY

Pan-Alberta Gas Ltd.

United States Gypsum

UPSTATE ENERGY INC.

TransCanada Gas Services Limited

Upstate Energy, Inc.

Niagara Falls, NY

- N/A

10

2.10

O N/A

$10 \quad 2.10$

US GEN NEW ENGLAND

PG\&E Energy Trading

New England Power Company

US GEN NEW ENGLAND

Renaissance Energy Ltd.

New England Power Company

Waddington, NY

$0 \quad$ N/A

$22 \quad 2.47$

$0 \quad$ N/A

$22 \quad 2.47$

US GEN NEW ENGLAND

TransCanada Gas Services limited

New England Power Company

Point
of

Waddington, NY

Waddington, NY

1260

2.87

1143

2.48

$20 \quad 2.54$

$2423 \quad 2.68$

Waddington, NY
$10 \quad 3.25$

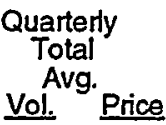

$276 \quad 1.78$

$9 \quad 2.45$

$7 \quad 2.26$

$186 \quad 2.63$

$273 \quad 2.71$

$65 \quad 2.70$

$585 \quad 2.69$ 


\section{SHORT-TERM SALES \\ YEAR: 1999 Quarter: Four \\ Estimated Volumes (MMCF) \& Prices (\$ / MMBTU)}

Importer

Seller

Purchaser / End User

UTILICORP UNITED INC.

Aquila Energy Marketing

Utilicorp United

UTILICORP UNITED INC.

CXY Marketing

Utilicorp United

UTILICORP UNITED INC.

Coral Energy Canada Inc.

Utilicorp United

UTILICORP UNITED INC

Premstar Energy Canada, Ltd.

Utilicorp United

UTILICORP UNITED INC

ProGas Limited

Utilicorp United

UTILICORP UNITED INC.

TransCanada Gas Services Limited

Utilicom United

UTILICORP UNITED INC

TransCanada Gas Services Limited

Utilicorp United

VERMONT GAS SYSTEMS, INC.

Enron Capital \& Trade Resources Canada

Vermont Gas Systems, Inc.

Point

of

Entry

October

Vol. Price

November

Vol. Price

December

Vol. Price

Quarterly

Total

Vol. Price

International Falls,

$357 \quad 2.64$

$0 \quad$ N/A

$0 \quad$ N/A

357

2.64

Noyes, Minnesota

$589 \quad 2.39$

$645 \quad 3.05$

$667 \quad 2.18$

1901

2.54

Intemational Falls,

$77 \quad 2.46$

47

2.97

$441 \quad 2.33$

$998 \quad 2.65$

Intemational Falls,

$7 \quad 2.45$

$0 \quad$ N/A

$0 \quad$ N/A

$7 \quad 2.45$

Noyes, Minnesota

$0 \quad$ N/A

150

3.06

$155 \quad 2.19$

$305 \quad 2.62$

Intemational Falls,

$3 \quad 2.42$

$10 \quad 2.99$

$15 \quad 2.14$

$28 \quad 2.49$

Noyes, Minnesota

o N/A

$75 \quad 3.09$

$24 \quad 2.22$

$99 \quad 2.88$

Highgate Spr., VT

o N/A

$45 \quad 2.61$

$70 \quad 2.21$

$115 \quad 2.37$

VERMONT GAS SYSTEMS, INC.

Gaz Metropolitain, Inc.

Vermont Gas Systems, Inc.

Highgate Spr., VT

o N/A

$35 \quad 2.10$

$0 \quad$ N/A

35

2.10

VERMONT GAS SYSTEMS, INC.

TransCanada Gas Services Limited

Vermont Gas Systems, Inc.

Highgate Spr., VT

$8 \quad 2.68$

$20 \quad 2.64$

$68 \quad 2.86$

95

2.80

WISCONSIN GAS COMPANY

CXY Marketing

Wisconsin Gas
Noyes, Minnesota

270

2.17

o N/A

270

2.17 
SHORT-TERM EXPORTERS

Estimated Volumes (MMCF)

AMOCO ENERGY TRADING CORPORATION APPLIED LNG TECHNOLOGIES USA, L.LC. BC GAS UTILITY LTD.

BIG SKY GAS MARKETING

BURLINGTON RESOURCES TRADING INC. COENERGY TRADING COMPANY

CONOCO INC.

CONSUMERS' GAS COMPANY LIMITED

COOK INLET ENERGY SUPPLY, L.P.

CORAL ENERGY RESOURCES, L.P.

DIST. DE GAS NATURAL DE MEXICAL

DUKE ENERGY TRADING AND MKTG SERVICES L.L.C.

DYNEGY MARKETING \& TRADE

EL PASO ENERGY MARKETING COMPANY

ENER-SON OF U.S.A.

ENGAGE ENERGY U.S., L.P.

ENRON CAPITAL \& TRADE RESOURCES CORP.

ERI SERVICES, INC.

HOWARD ENERGY MARKETING, INC.

KN MARKETING, L.P.

MEXICANA DE COBRE, S.A. DE C.V.

NESI ENERGY MARKETING, LLC.

NUI ENERGY BROKERS, INC.

ONYX GAS MARKETING COMPANY, L.C.

PEMEX GAS

PREMSTAR ENERGY CANADA, LTD.

QUESTAR ENERGY TRADING COMPANY

RELIANT ENERGY SERVICES, INC.

STAR NATURAL GAS COMPANY

TENASKA MARKETING VENTURES

TRANSCANADA GAS SERVICES INC.

TRISTAR GAS MARKETING COMPANY

UNION GAS LIMITED

UNION PACIFIC FUELS, INC.

WEST TEXAS GAS, INC.

WGR CANADA, INC.

WILLIAMS ENERGY SERVICES COMPANY

TOTALS
1998

Oct.-Dec.
1999 Jan-Mar

82

3

58

0

2315

734

808

1546

524

701

2707

2521

962

29

918

698

105

621

2093

0

152

7

10

2134

0

150

567

0

188

337

494

4594

1016

459

0

40

$\underline{27570}$

190

797

731

2241

1575

1525

66

1593

611

0

0

3088

0

0

0

0
4451

119

0

2045

0

319

0

53

5269

449

515

41

$\underline{26070}$
1999

Apr-Jun.
1999

Jul.-Sep.
1999

Oct.-Dec.

$\begin{array}{rrr}0 & 0 & 0 \\ 8 & 7 & 69 \\ 0 & 0 & 0 \\ 458 & 598 & 260 \\ 231 & 184 & 0 \\ 0 & 0 & 4388 \\ 0 & 0 & 0 \\ 0 & 0 & 0 \\ 0 & 0 & 0 \\ 1999 & 365 & 0 \\ 972 & 1017 & 1004 \\ 1137 & 1353 & 303 \\ 989 & 213 & 916 \\ 1035 & 1184 & 621 \\ 54 & 54 & 11 \\ 168 & 0 & 0 \\ 127 & 0 & 0 \\ 0 & 0 & 0 \\ 0 & 0 & 0 \\ 850 & 0 & 0 \\ 547 & 1038 & 570 \\ 0 & 0 & 0 \\ 0 & 0 & 0 \\ 33 & 0 & 0 \\ 7431 & 11575 & 10641 \\ 41 & 0 & 450 \\ 0 & 0 & 0 \\ 1605 & 0 & 0 \\ 0 & 0 & 22 \\ 0 & 0 & 3416 \\ 0 & 0 & 0 \\ 0 & 0 & 0 \\ 5287 & 5367 & 7537 \\ 0 & 0 & 0 \\ 482 & 454 & 541 \\ 0 & 0 & 0 \\ 68 & 0 & 0\end{array}$

23522 


\section{PURCHASERS OF SHORT-TERM EXPORTS}

Estimated Volumes Exported (MMCF)

ACCO

Amoco Canada Petroleum

Aquila Canada Corporation

Aquila Energy Marketing

Baja Oriente

BC Gas Inc.

C\&D Die Casting

Cibola Canada

CoEnergy Trading Company

Conagas

Coral Energy Canada Inc.

Coral Energy Resources

Cris-P

CXY Energy Marketing

Distribuidora de Gas Natural de Mexicali

Duke Energy Marketing Canada

Dynegy Canada Inc.

El Paso Energy Marketing Canada

EMC Gas Transmission, Inc.

Energy Dynamics

Engage Energy Canada L.P.

Enogex Services

Enron Capital \& Trade Resources Canada

ERI Services Canada Ltd.

Howard Energy Company

Koch Energy Trading

Mexcobre

Michigan Consolidated Gas Company

MidAmerican Energy Company

Milacron

National Fuel Gas Distribution

Naturál Gas Clearinghouse Canada, Inc.

NESI Energy Marketing

Nicor Energy

PEMEX

PFALTZGRAFF

PG\&E Energy Trading

Premstar Energy Canada Ltd.

Reliant Energy Services, Inc.

San Francisco Tile

Santa Fe Greenhouse

Sempra Energy Trading Corp.

St. Clair Pipelines Ltd.

Tenaska Marketing Ventures

TransCanada Gas Services Limited

Various British Columbia Customers

Various Ontario Customers

Various Saskatchewan Customers

WGR Canada, Inc.
1998

Oct.-Dec.
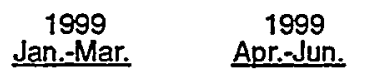

1999
Jul.-Sep.

1999

Oct.-Dec.

20

2324

$0 \quad 0$

$0 \quad 0$

03

$5 \quad 17$

$0 \quad 465$

$701 \quad 731$

465

125

1293

10

70

1657

0

$\begin{array}{rr}0 & 0 \\ 105 & 0\end{array}$

630

0

0

0

0

7

729

0

14868

0

81

0

$\begin{array}{ll}0 & \\ 3 & 5\end{array}$

$18 \quad 26$

$\begin{array}{rr}0 & 0 \\ 918 & 0\end{array}$

$\begin{array}{rl}0 & 0 \\ 382 & 0\end{array}$

$150 \quad 0$

$5590 \quad 5588$

$0 \quad 190$
16

21

13

0

24

0

41

4388

541

0

0

19

0

1004

0

759

621

1104

0

0

0

0

0

0

0

0

1038

0

020

$0 \quad 0$

$0 \quad 0$

$0 \quad 0$

$0 \quad 0$

$13770 \quad 10944$

$28 \quad 22$

$0 \quad 0$

$0 \quad 450$

$0 \quad 23$

42

$0 \quad 0$

$0 \quad 55$

0

0 6

012

$598 \quad 260$
$0 \quad 0$

00

$5367 \quad 10952$ 


\section{PURCHASERS OF SHORT-TERM EXPORTS}

Estimated Volumes Exported (MMCF)

\begin{tabular}{|c|c|c|c|c|}
\hline $\begin{array}{c}1998 \\
\text { Oct.-Dec. }\end{array}$ & $\begin{array}{c}1999 \\
\text { Jan.-Mar. }\end{array}$ & $\begin{array}{c}1999 \\
\text { Apr--Jun. }\end{array}$ & $\begin{array}{c}1999 \\
\text { Jul.-Sep. }\end{array}$ & $\begin{array}{c}1999 \\
\text { Oct.-Dec. }\end{array}$ \\
\hline
\end{tabular}




\section{SHORT-TERM SALES}

YEAR: 1999 Quarter: Four

Estimated Volumes (MMCF) \& Prices ( $\$$ /MMBTU)

Exporter

Seller

Purchaser/ End User

APPLIED LNG TECHNOLOGIES USA, L.L.C.

Applied LNG Technologies USA, L.L.C.

ACCO

APPLIED LNG TECHNOLOGIES USA, L.L.C.

Applied LNG Technologies USA, L.L.C.

Baja Oriente

APPLIED LNG TECHNOLOGIES USA, L.L.C.

Applied LNG Technologies USA, L.L.C.
Cris-P

APPLIED LNG TECHNOLOGIES USA, L.L.C.

Applied LNG Technologies USA, L.L.C.

PFALTZGRAFF

APPLIED LNG TECHNOLOGIES USA, L.L.C.

Applied LNG Technologies USA, L.LC.

San Francisco Tile

Nogales, Arizona

- N/A

$2 \quad 5.82$

o $\quad 5.82$

$2 \quad 5.82$

BIG SKY GAS MARKETING

Big Sky Gas Marketing

Various Saskatchewan Customers

Havre, Montana

$260 \quad 2.51$

o N/A

O N/A

260

2.51

COENERGY TRADING COMPANY

CoEnergy Trading Company

CoEnergy Trading Company

Detroit, Michigan

106

2.42

133

2.42

o N/A

238

2.42

COENERGY TRADING COMPANY

CoEnergy Trading Company

CoEnergy Trading Company

Marysville, Michigan

O N/A

$265 \quad 2.42$

309

2.42

574

2.42

COENERGY TRADING COMPANY

CoEnergy Trading Company

CoEnergy Trading Company

St. Clair, Michigan

o N/A

$1932 \quad 2.42$

$1644 \quad 2.42$

$3576 \quad 2.42$

DIST. DE GAS NATURAL DE MEXICALJ

Engage Energy US, L.P.

Distribuidora de Gas Natural de Mexical

Calexico, Califomia

$379 \quad 3.03$

$342 \quad 3.43$

283

2.72

$1004 \quad 3.08$

DUKE ENERGY TRADING AND MKTG SERVICES L.LC.

Duke Energy Trading and Marketing

PEMEX

Clint, Texas

$303 \quad 2.41$

$0 \quad$ N/A

$0 \quad$ N/A

303

2.41

DYNEGY MARKETING \& TRADE

Dynegy Marketing \& Trade

Cibola Canada

Port of Morgan, MT
- N/A

- N/A

$41 \quad 2.12$

41

2.12 
SHORT-TERM SALES

YEAR: 1999 Quarter: Four

Estimated Volumes (MMCF) \& Prices (\$ / MMBTU)

Exporter

Seller

Purchaser / End User

DYNEGY MARKETING \& TRADE

Dynegy Marketing \& Trade

Dynegy Canada inc.

DYNEGY MARKETING \& TRADE

Dynegy Marketing \& Trade

Dynegy Canada Inc.

DYNEGY MARKETING \& TRADE

Dynegy Marketing \& Trade

Enogex Services

DYNEGY MARKETING \& TRADE

Dynegy Marketing \& Trade

Koch Energy Trading

DYNEGY MARKETING \& TRADE

Dynegy Marketing \& Trade

MidAmerican Energy Company

DYNEGY MARKETING \& TRADE

Dynegy Marketing \& Trade

Reliant Energy Services, Inc.

DYNEGY MARKETING \& TRADE

Dynegy Marketing \& Trade

Sempra Energy Trading Corp.

DYNEGY MARKETING \& TRADE

Dynegy Marketing \& Trade

Tenaska Marketing Ventures

EL PASO ENERGY MARKETING COMPANY

El Paso Energy Marketing Company

El Paso Energy Marketing Canada

EL PASO ENERGY MARKETING COMPANY

El Paso Energy Marketing Company

El Paso Energy Marketing Canada

ENER-SON OF U.S.A.

ALT L.L.C.

ACCO

ENER-SON OF U.S.A.

ALT L.L.C.

Baja Oriente
Nogales, Arizona

Point

Exit

Port of Morgan, MT

St. Clair, Michigan

Port of Morgan, MT

Port of Morgan, MT

Port of Morgan, MT

Port of Morgan, MT

Port of Morgan, MT

Port of Morgan, MT

Marysville, Michigan

Niagara Falls, NY

50

Nogales, Arizona
16.66

$1 \quad 6.66$

- N/A

$2 \quad 6.66$

October

Vol. Price

November

December

Vol. Price

Quarterly

Total

Vol. ${ }^{\text {Avg. Price }}$

$0 \quad$ N/A

- N/A

$143 \quad 2.28$

143

2.28

$\begin{array}{llll}172 & 2.91 & 444 & 3.31\end{array}$

$0 \quad$ N/A

616

3.20

o N/A

O N/A

$10 \quad 2.12$

10

2.12

o N/A

O N/A

$2 \quad 2.12$

$2 \quad 2.12$

$0 \quad$ N/A

- N/A

$20 \quad 2.12$

20

2.12

$0 \quad$ N/A

O N/A

$23 \quad 2.12$

$23 \quad 2.12$

o N/A

o N/A

$55 \quad 2.12$

$55 \quad 2.12$

o N/A

- N/A

$6 \quad 2.12$

$6 \quad 2.12$

o N/A

101

2.30

$16 \quad 3.09$

117

2.41

$203 \quad 2.63$

o N/A

$0 \quad$ N/A

27.49

- N/A

o N/A

$2 \quad 7.49$
Vol. Price

$503 \quad 2.63$ 


\section{SHORT-TERM SALES \\ YEAR: 1999 Quarter: Four \\ Estimated Volumes (MMCF) \& Prices (\$ / MMBTU)}

Exporter

Seller

Purchaser / End User

ENER-SON OF U.S.A.

ALTL.L.C.

Cris-P

ENER-SON OF U.S.A.

ALT L.L.C.

PFALTZGRAFF

MEXICANA DE COBRE, S.A. DE C.V.

MGI Supply

Mexcobre

PEMEX GAS

MGI Supply

PEMEX

PEMEX GAS

MGI Supply

PEMEX

Douglas, Arizona

El Paso, Texas

Hidalgo, Texas

St. Clair, Michigan

St. Clair, Michigan

Detroit, Michigan

Detroit, Michigan

$0 \quad$ N/A

$6 \quad 3.45$

$\begin{array}{ll}6 & 2.48\end{array}$

$12 \quad 2.97$

TENASKA MARKETING VENTURES

Tenaska Marketing Ventures

Various Ontario Customers

\section{October November December}

Vol. Price Vol. Price Vol. Price

$\begin{array}{llllll}2 & 7.67 & 0 & \text { N/A } & 0 & \text { N/A }\end{array}$

$2 \quad 7.67$

$6 \quad 7.49$

$0 \quad N / A$

$0 \quad$ N/A

$6 \quad 7.49$

183

2.67

$176 \quad 3.04$

211

2.38

$570 \quad 2.68$

$\begin{array}{llllllll}2109 & 2.64 & 3581 & 2.84 & 2791 & 2.21 & 8481 & 2.58\end{array}$

162

2.11

$254 \quad 2.23$

$616 \quad 2.29$

$496 \quad 2.75$

$\begin{array}{ll}566 & 3.02\end{array}$

$240 \quad 2.53$

$1302 \quad 2.83$

$242 \quad 2.85$

O N/A

$0 \quad$ N/A

$242 \quad 2.85$

CMS Marketing

PREMSTAR ENERGY CANADA, LTD.

Premstar Energy Canada Ltd.

STAR NATURAL GAS COMPANY

Carthage

STAR NATURAL GAS COMPANY

TXU

- N/A

$5 \quad 3.45$

$\begin{array}{ll}5 & 2.48\end{array}$

$10 \quad 2.97$

St. Clair, Michigan $\begin{array}{llllllll}0 & \text { N/A } & 1931 & 3.35 & 1485 & 2.16 & 3416 & 2.83\end{array}$ 


\section{SHORT-TERM SALES \\ YEAR: 1999 Quarter: Four \\ Estimated Volumes (MMCF) \& Prices (\$ / MMBTU)}

Exporter

Seller

Purchaser/End User

UNION GAS LIMITED

Amoco Energy Trading Corporation

Various Ontano Customers

\section{UNION GAS LIMITED}

Anadarko Trading Company

Various Ontario Customers

UNION GAS LIMITED

Dynegy Marketing \& Trade

Various Ontario Customers

UNION GAS LIMITED

Engage Energy US, L.P.

Various Ontano Customers

UNION GAS LIMITED

PG\&E Energy Trading

Various Ontanio Customers

UNION GAS LIMITED

Proliance

Various Ontario Customers

UNION GAS LIMITED

Reliant Energy Services, Inc.

Various Ontario Customers

UNION GAS LIMITED

Williams Energy Services Company

Various Ontano Customers

WEST TEXAS GAS, INC.

West Texas Gas, Inc.

Conagas

\section{Point \\ Exit}

Detroit, Michigan

Detroit, Michigan

Detroit, Michigan

Detroit, Michigan

Detroit, Michigan

Detroit, Michigan

Detroit, Michigan

Detroit, Michigan

Eagle Pass, Texas

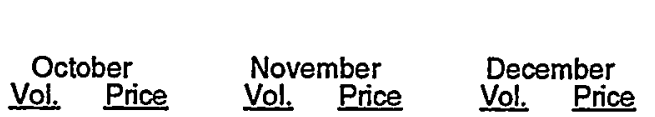

Quarterly

Total

Vol. Price

$327 \quad 2.50$

- N/A

$0 \quad$ N/A

327

2.50

o N/A

$157 \quad 3.05$

$0 \quad$ N/A

157

3.05

$164 \quad 2.51$

- N/A

- N/A

$\begin{array}{ll}164 & 2.51\end{array}$

$819 \quad 250$

$944 \quad 2.92$

$952 \quad 2.54$

$2715 \quad 2.66$

o N/A

63

2.94

652

2.07

$1282 \quad 2.50$

o N/A

158

2.51

$158 \quad 2.51$

315

2.51

788

2.95

97

2.28

2255

2.62

o N/A

158

3.01

163

3.05

321

3.03

$173 \quad 2.64$

174

3.13

19

$194 \quad 2.22$

54

2.65 